\title{
Synergy Between Experimental and Computational Chemistry Reveals the Mechanism of Decomposition of Nickel-Ketene Complexes
}

\author{
Nicholas D. Staudaher, Atta M. Arif, and Janis Louie* \\ Department of Chemistry, the University of Utah, 315 S 1400 E, Salt Lake City, Utah 84112. \\ Louie@chem.utah.edu \\ SUPPORTING INFORMATION
}

1. General Experimental

2. NMR Scale Reactions

3. Synthesis of Ketene Complexes

4. Kinetics

5. Computational Details

6. X-Ray Data Table of Contents

7. X-Ray Data

8. References

9. NMR Spectra
S1

S2

S2

S9

S9

S61

S62

S173

S174

General Considerations All reactions were carried out under an atmosphere of $\mathrm{N}_{2}$ in a glovebox. All glassware was oven dried. Benzene and pentane were sparged with nitrogen, dried over neutral alumina and deoxygenated over Q5 under $\mathrm{N}_{2}$ using a Grubbs type solvent purification system. Dppf, dppe, dppp, dppb, dppbenzene (strem) triphenylphosphine, 1,3,5-trimethoxybenzene (sigma), and $n$-butyl bromide- $d_{9}$ (Cambridge Isotope) were purchased and used as received. $\mathrm{Ni}(\mathrm{COD})_{2}$ was purchased from Strem or synthesized according to the literature procedure. ${ }^{1}$ Diphenylketene ${ }^{2}$ and aryl alkyl ketenes ${ }^{3}$ were prepared according to the literature procedures. Deuterated benzene (sigma), deuterated toluene and deuterated THF (Cambridge isotope), were distilled from $\mathrm{CaH}_{2}$ and degassed by three freeze pump thaw cycles. Screw-cap NMR tubes were used for all NMRs. The tubes were cleaned by rinsing with acetone, 
sonicating with 1:1 THF/concentrated $\mathrm{HCl}$ for $1 \mathrm{~h}$ to remove residual $\mathrm{Ni}$, and rinsed with water. ${ }^{1} \mathrm{H}$ Nuclear Magnetic Resonance spectra were acquired at 300, 400, or $500 \mathrm{MHz} .{ }^{13} \mathrm{C}$ spectra were recorded at 100 or $125 \mathrm{MHz} .{ }^{31} \mathrm{P}$ spectra were recorded at $121 \mathrm{MHz}$. All ${ }^{13} \mathrm{C}$ and ${ }^{31} \mathrm{P}$ NMR spectra were proton decoupled. ${ }^{1} \mathrm{H}$ and ${ }^{13} \mathrm{C}$ spectra were referenced to residual solvent peaks $\left(\mathrm{C}_{6} \mathrm{D}_{6} \delta 7.15\right.$ and $\delta 128.6$; THF$\mathrm{d}_{8}, \delta 3.76$ and $\delta 68.0$, respectively). ${ }^{31} \mathrm{P}$ NMR shifts were reported with respect to external $85 \% \mathrm{H}_{3} \mathrm{PO}_{4}(0$ ppm). IR spectra were recorded on a Bruker Tensor 27 FT-IR spectrometer. X-ray crystallography data was collected and analyzed by Dr. Atta Arif at the University of Utah. Elemental analysis was performed by Midwest Microlabs LLC.

Reaction of $\mathbf{P P h}_{3}, \mathbf{N i}(\mathbf{C O D})_{2}$, and phenyl butyl ketene: A solution of $\mathrm{PPh}_{3}(19.6 \mathrm{mg}, 0.075 \mathrm{mmol})$ in $1.5 \mathrm{~mL} \mathrm{C}_{6} \mathrm{D}_{6}$ was added to $\mathrm{Ni}(\mathrm{COD})_{2}(20.4 \mathrm{mg}, 0.075 \mathrm{mmol})$ and the solution stirred for 5 minutes. An aliquot $(0.75 \mathrm{~mL}, 0.0375 \mathrm{mmol})$ was added to phenyl butyl ketene $(19.6 \mathrm{mg}, 3 \mathrm{eq})$ and the two solutions stirred for $2 \mathrm{~h}$, at which point they were analyzed by ${ }^{31} \mathrm{P}$ and ${ }^{1} \mathrm{H}$ NMR. The solution containing no ketene displayed only a sharp singlet at $40.0 \mathrm{ppm}$ in the ${ }^{31} \mathrm{P}$ NMR, consistent with $\left(\mathrm{PPh}_{3}\right)_{2} \mathrm{Ni}(\mathrm{COD})$. The ${ }^{1} \mathrm{H}$ NMR spectrum was consistent with a $1: 1$ mixture of $\left(\mathrm{PPh}_{3}\right)_{2} \mathrm{Ni}(\mathrm{COD})$ and free cyclooctadiene. The spectrum of the solution containing ketene had two broad singlets at 41.6 and 33.8, indicating that the $\left(\mathrm{PPh}_{3}\right)_{2} \mathrm{Ni}(\mathrm{COD})$ had been consumed. ${ }^{1} \mathrm{H}$ NMR of this sample was not possible due to paramagnetic contaminants. IR spectroscopy revealed six carbonyl peaks, indicative of several carbonyl complexes, including cis- $\left(\mathrm{PPh}_{3}\right)_{2} \mathrm{Ni}(\mathrm{CO})_{2}$.

Reaction of $\mathrm{Ni}(\mathrm{COD})_{2}$, dppe, and diphenylketene: A solution of $\mathrm{Ni}(\mathrm{COD})_{2}(22.5 \mathrm{mg}, 0.0818 \mathrm{mmol})$ and dppe (32.6 mg, $0.0818 \mathrm{mmol}$ ) in $\mathrm{C}_{6} \mathrm{H}_{6}$ was stirred for 5 minutes and added to diphenylketene (23.8 $\mathrm{mg}, 0.123 \mathrm{mmol}, 1.5$ equiv) and stirred overnight. ${ }^{31} \mathrm{P}$ NMR revealed a singlet at $44.7 \mathrm{ppm}$, consistent with (dppe) ${ }_{2} \mathrm{Ni}$.

Reaction of $\mathrm{Ni}(\mathrm{COD})_{2}$ with dppe, dppb, dppb, and dppbenzene: $\mathrm{Ni}(\mathrm{COD})_{2}(22.5 \mathrm{mg}, 0.0818 \mathrm{mmol})$ and an equimolar amount of bidentate phosphine were dissolved in $\mathrm{C}_{6} \mathrm{H}_{6}(0.75 \mathrm{mmol})$ and stirred for $4 \mathrm{~h}$ before being analyzed by ${ }^{31} \mathrm{P}$ NMR. Dppe resulted in two singlets at 51.3 and $44.6 \mathrm{ppm}$, the second of which is consistent with (dppe) ${ }_{2} \mathrm{Ni}^{4}$ while dppp gave two singlets at 21.3 and $12.7 \mathrm{ppm}$ (the second of these is consistent with $\left.(\mathrm{dppp})_{2} \mathrm{Ni}\right){ }^{5} \mathrm{dppb}$ gave singlets at 35.5 and $17.6 \mathrm{ppm}$, and dppbenzene displayed singlets at 55.8 and $47.8 \mathrm{ppm}$.

(dppf)Ni(COD) was prepared according to a modified literature procedure. $\mathrm{Ni}(\mathrm{COD})_{2}(496.2 \mathrm{mg}, 1.8$ mmol, 1 equiv) was weighed into a $20 \mathrm{~mL}$ scintillation vial. Dppf (1.000 g, $1.8 \mathrm{mmol}, 1$ equiv) was weighed into a $4 \mathrm{~mL}$ vial and transferred to the vial containing $\mathrm{Ni}(\mathrm{COD})_{2}$. Residual dppf was washed into the $20 \mathrm{~mL}$ vial with $5 \mathrm{x} 2 \mathrm{~mL} \mathrm{C}_{6} \mathrm{H}_{6}$. Copious orange precipitate forms immediately. The reaction was 
stirred for $2.5 \mathrm{~h}$, and pentane $(10 \mathrm{~mL})$ was added. After an additional hour, the solids were collected by filtration, washed 3x $10 \mathrm{~mL}$ pentane, dried under vacuum, and transferred to a tared vial to yield $1.1527 \mathrm{~g}$, $89 \%$ of an orange solid. ${ }^{1} \mathrm{H}$ and ${ }^{13} \mathrm{C}$ NMR data agree with the literature. ${ }^{6}$

General procedure for setup of (Dppf)Ni(Ketene) Complexes: In the drybox, $\mathrm{Ni}(\mathrm{COD})_{2}(100 \mathrm{mg}$, $0.363 \mathrm{mmol}, 1 \mathrm{eq})$ and dppf (201.5 $\mathrm{mg}, 0.363 \mathrm{mmol}, 1 \mathrm{eq})$ were weighed into a $20 \mathrm{~mL}$ scintillation vial. Benzene $(3 \mathrm{~mL})$ was added and the solution stirred for 2 minutes, at which point ketene $(1.09 \mathrm{mmol}, 3 \mathrm{eq})$ was added in $2 \mathrm{~mL}$ benzene.

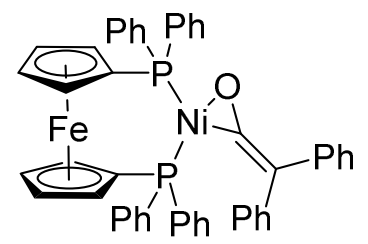

(Dppf)Ni(diphenylketene), 8a was synthesized according to the general procedure using diphenylketene (141.2 $\mathrm{mg}, 0.727 \mathrm{mg}, 2 \mathrm{eq})$. The reaction was stirred for $24 \mathrm{~h}$ and pentane $(15 \mathrm{~mL})$ was added. After an additional $24 \mathrm{~h}$ the solids were collected by filtration, washed $3 \times 10 \mathrm{~mL}$ pentane and dried under vacuum to yield $249.5 \mathrm{mg}$ ( $85 \%$ yield) of an orange powder. Crystals suitable for x-ray crystallography were obtained by allowing a $1 / 3$ scale reaction to sit unstirred for $48 \mathrm{~h}$, followed by diffusion of pentane into the reaction mixture. ${ }^{1} \mathrm{H}$ NMR $\left(400 \mathrm{MHz}, \mathrm{C}_{6} \mathrm{D}_{6}\right): \delta 8.12(\mathrm{~d}, J=8 \mathrm{~Hz}, 2 \mathrm{H}), 8.05(\mathrm{t}, J=10 \mathrm{~Hz}, 4 \mathrm{H})$, $7.84(\mathrm{dd}, J=8 \mathrm{~Hz}, 12 \mathrm{~Hz}, 4 \mathrm{H}), 7.31$ (t, $J=8 \mathrm{~Hz}, 2 \mathrm{H}), 7.12(\mathrm{~s}, 1 \mathrm{H}), 7.00$ (m, $14 \mathrm{H}), 6.62(\mathrm{t}, J=8 \mathrm{~Hz}$, $1 \mathrm{H}), 6.53(\mathrm{t}, J=8 \mathrm{~Hz}, 2 \mathrm{H}), 4.26(\mathrm{~d}, 2 \mathrm{H}), 3.86(\mathrm{~s}, 2 \mathrm{H}), 3.72(\mathrm{~d}, 2 \mathrm{H}), 3.63(\mathrm{~s}, 2 \mathrm{H}) .{ }^{13} \mathrm{C}\left\{{ }^{1} \mathrm{H}\right\} \mathrm{NMR}(100.6$ $\left.\mathrm{MHz}, \mathrm{C}_{6} \mathrm{D}_{6}\right): \delta 168.32(\mathrm{dd}, J=44,7 \mathrm{~Hz}, \mathrm{O}=C=\mathrm{C}) 142.73(\mathrm{~d}, J=3 \mathrm{~Hz}), 142.34(\mathrm{~d}, J=8 \mathrm{~Hz}), 125.95(\mathrm{~d}, J$ $=34 \mathrm{~Hz}), 135.65(\mathrm{~d}, J=14), 135.33(\mathrm{~d}, J=14 \mathrm{~Hz}), 134.25$ (dd, $J=2 \mathrm{~Hz}, 41 \mathrm{~Hz}), 133.64(\mathrm{~s}), 130.87$ (dd, $J=2 \mathrm{~Hz}, 9 \mathrm{~Hz}), 129.26$ (d, $J=9 \mathrm{~Hz}), 127.61$ (s), 124.92 (s), 123.09 (s), 86.14 (d, $J=56 \mathrm{~Hz}), 76.06$ (d, $J$ $=44 \mathrm{~Hz}), 75.08(\mathrm{~d}, J=11 \mathrm{~Hz}), 74.67(\mathrm{~d}, J=9 \mathrm{~Hz}), 73.36(\mathrm{~d}, J=5 \mathrm{~Hz}), 71.95(\mathrm{~d}, J=5 \mathrm{~Hz}) .{ }^{31} \mathrm{P}\left\{{ }^{1} \mathrm{H}\right\}$ NMR (121 MHz, $\left.\mathrm{C}_{6} \mathrm{D}_{6}\right): \delta 39.59$ (d, $\left.J=19.4 \mathrm{~Hz}\right), 17.65$ (d, $J=20.6 \mathrm{~Hz}$ ). IR (nujol, NaCl): 2351 (w, CC), 1614 (s), 1581 (s, CO), 1488 (s), 1307 (m), 1292 (m), 1235 (s), 1201 (w), 1169 (m), 1157 (m), 1098 (s), 1071 (m), 1028 (m), 999 (m), 930 (m), 898 (w), 866 (w), 841 (w), 822 (m) cm². Several attempts gave elemental analyses that were low in both carbon and hydrogen.

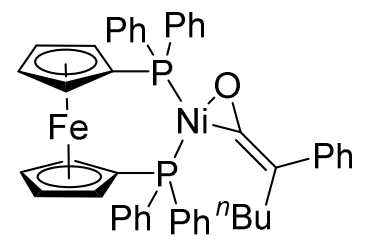

(Dppf)Ni(butylphenylketene), $8 \mathbf{b}$ was prepared according to the general procedure using phenyl butyl ketene (126.7 mg, $0.727 \mathrm{mmol}, 2$ equiv). The reaction was stirred for $36 \mathrm{~h}$, at which point the reaction was a yellow-orange suspension. The solids were collected by filtration, washed $3 \times 10 \mathrm{~mL}$ pentane, and dried under vacuum to yield $226.1 \mathrm{mg}, 79 \%$ of a yellow powder. Crystals suitable for x-ray crystallography were obtained by allowing a $1 / 3$ scale reaction to sit unstirred for $48 \mathrm{~h}$, followed by diffusion of pentane into the reaction mixture. ${ }^{1} \mathrm{H} \mathrm{NMR}\left(400 \mathrm{MHz}, \mathrm{C}_{6} \mathrm{D}_{6}\right): \delta 8.23(\mathrm{~d}, J=8 \mathrm{~Hz}, 2 \mathrm{H}), 8.11$ 
(m, 4H), $8.00(\mathrm{t}, J=8,4 \mathrm{H}), 7.45(\mathrm{t}, J=8 \mathrm{~Hz}, 2 \mathrm{H}), 7.00-7.07(\mathrm{~m}, 13 \mathrm{H}), 4.29(\mathrm{~d}, 2 \mathrm{H}), 3.89(\mathrm{~s}, 2 \mathrm{H}), 3.74$ $(\mathrm{d}, 2 \mathrm{H}), 3.66(\mathrm{~s}, 2 \mathrm{H}), 2.15(\mathrm{t}, J=8 \mathrm{~Hz}, 2 \mathrm{H}), 1.36-1.26(\mathrm{~s}, 2 \mathrm{H}), 0.59(\mathrm{~s}, 5 \mathrm{H}) .{ }^{13} \mathrm{C}\left\{{ }^{1} \mathrm{H}\right\} \mathrm{NMR}(125 \mathrm{MHz}$, THF- $\left.d_{8}\right): 168.6(\mathrm{dd}, \mathrm{J}=41,9 \mathrm{~Hz}, \mathrm{O}=C=\mathrm{C}), 140.9$ (d, J = 8 Hz), 136.7 (d, J = 34 Hz), 136.4 (d, J = $\left.15 \mathrm{~Hz}\right)$, $135.8(\mathrm{~d}, \mathrm{~J}=13 \mathrm{~Hz}), 135.7$ (dd, J = 41, $3 \mathrm{~Hz}), 131.6$ (dd, J = 69, $1 \mathrm{~Hz}$ ), 129.9 (d, J = $10 \mathrm{~Hz}), 129.7$ (s), 129.6 (d, J = $10 \mathrm{~Hz}$ ), 128.6 (s), 126.0 (s), 121.7 (s), 85.6 (dd, J = 49, $6 \mathrm{~Hz}$ ), 79.1 (dd, J = 8, $6 \mathrm{~Hz}$, $\mathrm{O}=\mathrm{C}=C), 76.9(\mathrm{~d}, \mathrm{~J}=44 \mathrm{~Hz}), 75.9(\mathrm{~d}, \mathrm{~J}=11 \mathrm{~Hz}), 75.4(\mathrm{~d}, \mathrm{~J}=8 \mathrm{~Hz}), 74.3(\mathrm{~d}, \mathrm{~J}=6 \mathrm{~Hz}), 72.5(\mathrm{~d}, \mathrm{~J}=6 \mathrm{~Hz})$, 32.9 (d, J = 4 Hz), 31.3 (s), 27.0 (s), 16.4 (s). ${ }^{31} \mathrm{P}\left\{{ }^{1} \mathrm{H}\right\}$ NMR (121 MHz, $\left.\mathrm{C}_{6} \mathrm{D}_{6}\right): \delta 42.03$ (d, $\left.J=23 \mathrm{~Hz}\right)$, 17.10 (d, $J=23 \mathrm{~Hz}$ ). IR (nujol, NaCl): 1911 (w), 1657 (w), 1624 (s, CO), 1584 (s), 1491 (s), 1479 (s), 1435 (s), 1337 (w), 1306 (m), 1267 (s), 1213 (m) 1196 (m), 1184 (s) 1166 (s), 1093 (s), 1075 (w), 1031 (s), $1014(\mathrm{~m}), 998$ (m), $913(\mathrm{w}), 891$ (w), $834(\mathrm{~m}), 824$ (m), 791 (w) cm $\mathrm{cm}^{-1}$. Anal. Calcd. for $\mathrm{C}_{46} \mathrm{H}_{42} \mathrm{FeNiOP}_{2}$ : C, 70.17; H, 5.23. Found: C, 70.30; H, 5.45.

$\boldsymbol{n}$-Butyl Phenyl Ketene- $\boldsymbol{d}_{\boldsymbol{9}}$ was synthesized according to the literature procedure ${ }^{3}$ starting from $n$-butyl bromide- $d_{9}$ instead of $n$-butyl bromide. $4.18 \mathrm{~g}$ of acyl chloride were used to synthesize the ketene, of which $1.74(50 \%)$ was isolated with $>95 \%$ deuteration. ${ }^{1} \mathrm{H}$ NMR $\left(500 \mathrm{MHz}, \mathrm{C}_{6} \mathrm{D}_{6}\right): 7.09$ (t, $J=10 \mathrm{~Hz}$, 2H), $6.90(\mathrm{~m}, 3 \mathrm{H}), 2.00(\mathrm{t}, J=7.5 \mathrm{~Hz}, 0.08 \mathrm{H}), 1.25(\mathrm{~m}, 0.10 \mathrm{H}), 1.16(\mathrm{~m}, 0.09 \mathrm{H}), 0.74(\mathrm{t}, J=7.5 \mathrm{~Hz}$, $0.12 \mathrm{H})$.

(Dppf)Ni(butylphenylketene- $\boldsymbol{d}_{\mathbf{9}}$ ), 8b- $\boldsymbol{d}_{\mathbf{9}}$ was prepared in the same fashion as $\mathbf{8 b}$ with $n$-butyl phenyl ketene- $d_{9}$ on the same scale. $206.3 \mathrm{mg}(72 \%)$ of an orange powder was isolated with $>95 \%$ deuteration. ${ }^{1} \mathrm{H}$ NMR (500 MHz, $\left.\mathrm{C}_{6} \mathrm{D}_{6}\right): \delta 8.23(\mathrm{~d}, J=8 \mathrm{~Hz}, 2 \mathrm{H}), 8.11(\mathrm{~m}, 4 \mathrm{H}), 8.00(\mathrm{~m}, 4 \mathrm{H}), 7.45(\mathrm{t}, J=8 \mathrm{~Hz}, 2 \mathrm{H})$, 7.00-7.07 (m, 13H), 4.29 (d, 2H), $3.89(\mathrm{~s}, 2 \mathrm{H}), 3.74(\mathrm{~d}, 2 \mathrm{H}), 3.66(\mathrm{~s}, 2 \mathrm{H}), 2.15(0.05 \mathrm{H}), 1.36-1.26(0.16$ H), $0.59(0.1 \mathrm{H})$.

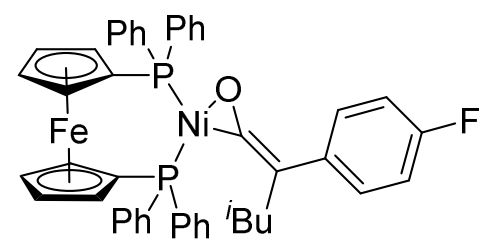

(Dppf)Ni(p-fluorophenyl isobutyl ketene), 8ca was synthesized according to the general procedure with $p$-fluorophenyl isobutyl ketene (129.5 mg, $0.727 \mathrm{mmol}, 2$ equiv). The reaction was stirred for $2.5 \mathrm{~h}$, pentane $(15 \mathrm{~mL})$ was added and the mixture was stored at $-40{ }^{\circ} \mathrm{C}$ overnight. The solids were collected by filtration, washed $4 \times 10 \mathrm{~mL}$ pentane, and dried under vacuum. The product was isolated as a red solid $(222.5 \mathrm{mg}, 79 \%)$. Crystals suitable for X-ray crystallography were grown by layering $1 \mathrm{~mL}$ of a saturated benzene solution of the complex with $0.5 \mathrm{~mL}$ benzene then $2.5 \mathrm{~mL}$ pentane. ${ }^{1} \mathrm{H}$ NMR $\left(400 \mathrm{MHz}, \mathrm{C}_{6} \mathrm{D}_{6}\right): \delta 8.07(\mathrm{~m}, 3.90 \mathrm{H}), 7.98(\mathrm{~m}, 3.79 \mathrm{H}), 7.93(\mathrm{~m}, 2.21 \mathrm{H}), 7.11$ (br, 5.49H), 7.05-6.96 (m, br, 9.36H), $4.26(\mathrm{~d}, J=5 \mathrm{~Hz}, 1.74 \mathrm{H}), 4.24$ (br, 0.26H), 3.91 (br, $1.75 \mathrm{H}), 3.86$ (br, 0.25H), 3.77 (br, 0.25H), 3.74 (br, 1.74H), 3.65 (br, 1.97H), 2.01 (d, $J=5 \mathrm{~Hz}, 1.79 \mathrm{H}), 1.91$ (m, $J=5 \mathrm{~Hz}$, $0.86 \mathrm{H}), 1.15(\mathrm{~d}, J=5 \mathrm{~Hz}, 0.79 \mathrm{H}), 0.49$ (d, $J=5 \mathrm{~Hz}, 5.62 \mathrm{H}) .{ }^{13} \mathrm{C}\left\{{ }^{1} \mathrm{H}\right\} \mathrm{NMR}\left(100.6 \mathrm{MHz}, \mathrm{C}_{6} \mathrm{D}_{6}\right): \delta 169.1$ $\left(\mathrm{ddd}, J_{\mathrm{PC}}=41,8 \mathrm{~Hz}, J_{\mathrm{CF}}=1 \mathrm{~Hz}\right), 160.5\left(\mathrm{~d}, J_{\mathrm{CF}}=238 \mathrm{~Hz}\right), 160.0\left(\mathrm{~d}, J_{\mathrm{CF}}=245 \mathrm{~Hz}\right), 137.4(\mathrm{dd}, J=8,3$ 
Hz), 136.4 (s), 136.0 (d, $J=34 \mathrm{~Hz}), 135.7$ (d, $J=15 \mathrm{~Hz}), 135.6$ (d, $J=18 \mathrm{~Hz}), 135.31$ (d, $J=32 \mathrm{~Hz})$, 135.26 (d, $J=14 \mathrm{~Hz}), 134.9$ (d, $J=2 \mathrm{~Hz}), 131.049$ (d, $J=2 \mathrm{~Hz}), 131.046$ (dd, $J=50,2 \mathrm{~Hz}), 129.9$ (d, $J$ $=7 \mathrm{~Hz}), 129.4(\mathrm{~d}, J=10 \mathrm{~Hz}), 129.2(\mathrm{~d}, J=10 \mathrm{~Hz}), 129.1(\mathrm{~s}), 128.9(\mathrm{~s}), 128.7(\mathrm{~s}), 128.5$ (s) these three peaks overlap with the residual solvent peak, $127.8(\mathrm{~d}, J=6 \mathrm{~Hz}), 116.1(\mathrm{~d}, J=21 \mathrm{~Hz}), 115.8(\mathrm{~d}, J=21$ $\mathrm{Hz}), 115.3(\mathrm{~d}, J=20 \mathrm{~Hz}), 114.6(\mathrm{~d}, J=20 \mathrm{~Hz}), 85.9(\mathrm{dd}, J=42,5 \mathrm{~Hz}), 76.8(\mathrm{~d}, J=44 \mathrm{~Hz}), 76.7$ (dd, $J=$ $8,6 \mathrm{~Hz}), 75.3(\mathrm{~d}, J=11 \mathrm{~Hz}), 75.0(\mathrm{~d}, J=10 \mathrm{~Hz}), 74.8(\mathrm{~d}, J=9 \mathrm{~Hz}), 74.6(\mathrm{~s})$ possibly a doublet that overlaps with the previous peak, $73.4(\mathrm{~d}, J=6 \mathrm{~Hz}), 73.3(\mathrm{~d}, J=6 \mathrm{~Hz}), 72.0(\mathrm{~d}, J=5 \mathrm{~Hz}), 71.7(\mathrm{~d}, J=5$ Hz), 44.8 (d, $J=6 \mathrm{~Hz}), 41.1$ (d, $J=4 \mathrm{~Hz}), 33.7$ (s), 32.4 (s), 30.7 (s), 29.1 (s), 26.9 (s), 23.6 (s), 23.3 (s), $23.2(\mathrm{~s}), 23.1$ (s). ${ }^{31} \mathrm{P}\left\{{ }^{1} \mathrm{H}\right\}$ NMR (121 MHz, $\left.\mathrm{C}_{6} \mathrm{D}_{6}\right): \delta 40.8(\mathrm{~d}, J=22 \mathrm{~Hz}), 40.2(\mathrm{~d}, J=21 \mathrm{~Hz}), 23.5(\mathrm{~s})$, 17.3 (d, $J=22 \mathrm{~Hz}), 17.0$ (d, $J=21 \mathrm{~Hz}$ ). IR (nujol, NaCl): 1743 (s), 1597 (w), 1505 (s), 1436 (s), 1306 (m), 1216 (m), 1611 (s), 1032 (m), 885 (w), 837 (w), 748 (w), 723 (w), 696 (s), 676 (m), 637 (w) cm ${ }^{-1}$. Anal. Calcd. for $\mathrm{C}_{46} \mathrm{H}_{41} \mathrm{FFeNiOP}_{2}$ : C, 68.61; H, 5.13. Found: C, 68.97; H, 5.40.

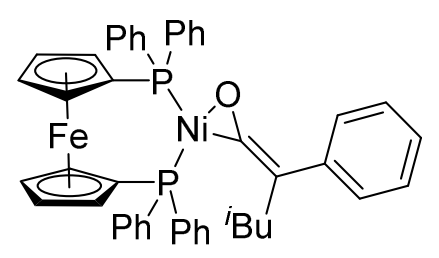

(Dppf)Ni(phenyl isobutyl ketene), 8cb was synthesized according to the general procedure using phenyl isobutyl ketene $(126.7 \mathrm{mg}, 0.727 \mathrm{mmol}, 2$ equiv). The reaction was stirred for $24 \mathrm{~h}$, at which point some orange precipitate had formed. Pentane $(15 \mathrm{~mL})$ was added, and precipitation was complete after $4 \mathrm{~h}$. At this point, the solids were collected by filtration, washed 3x $10 \mathrm{~mL}$ pentane, and dried under vacuum to yield $191.7 \mathrm{mg}, 79 \%$ of an orange powder. Crystals suitable for X-ray crystallography were grown by layering $1 \mathrm{~mL}$ of a saturated benzene solution of the complex with $0.5 \mathrm{~mL}$ benzene then $2.5 \mathrm{~mL}$ pentane. ${ }^{1} \mathrm{H}$ NMR $\left(300 \mathrm{MHz}, \mathrm{C}_{6} \mathrm{D}_{6}\right): \delta 8.15(\mathrm{~d}, J=8 \mathrm{~Hz}, 2 \mathrm{H}), 8.10(\mathrm{~m}, \mathrm{br}, 4 \mathrm{H}), 8.00$ (td, $(J=8,4 \mathrm{~Hz}, 4 \mathrm{H}), 7.40(\mathrm{t}, J=8 \mathrm{~Hz}, 2 \mathrm{H}), 7.1(\mathrm{~m}, 6 \mathrm{H}), 7.00(\mathrm{~m}, 7 \mathrm{H}), 4.27(\mathrm{~s}, 2 \mathrm{H}), 3.91(\mathrm{~s}, 2 \mathrm{H}), 3.74$ (s, 2H), 3.65 (s, 2H), 2.10 (d, $J=4 \mathrm{~Hz}, 2 \mathrm{H}), 2.03$ (pent, $J=4 \mathrm{~Hz}, 1 \mathrm{H}), 0.50(\mathrm{~d}, J=4 \mathrm{~Hz}, 6 \mathrm{H}) .{ }^{13} \mathrm{C}\left\{{ }^{1} \mathrm{H}\right\}$ NMR (125 MHz, $\left.\mathrm{C}_{6} \mathrm{D}_{6}\right): \delta 169.4(\mathrm{~d}, J=40,8 \mathrm{~Hz}, \mathrm{O}=C=\mathrm{C}), 141.4(\mathrm{~d}, J=7 \mathrm{~Hz}), 136.1(\mathrm{~d}, J=34 \mathrm{~Hz})$, 135.7 (d, $J=15 \mathrm{~Hz}), 135.3$ (d, $J=14 \mathrm{~Hz}), 135.1$ (dd, $J=40,2 \mathrm{~Hz}), 131.0$ (dd, $J=51,1 \mathrm{~Hz}), 129.4(\mathrm{~d}, J=$ $10 \mathrm{~Hz}$ ), 129.2 (d, $J=9 \mathrm{~Hz}), 129.1$ (s), 128.7 (m, overlaps with solvent), 126.9 (s), 122.5 (s), 86.2 (dd, $J=$ 48, $6 \mathrm{~Hz}), 77.6(\mathrm{dd}, J=8,5 \mathrm{~Hz}, \mathrm{O}=\mathrm{C}=C), 77.0(\mathrm{~d}, J=44 \mathrm{~Hz}), 75.3(\mathrm{~d}, J=11 \mathrm{~Hz}), 74.8(\mathrm{~d}, J=9 \mathrm{~Hz}), 73.3$ $(\mathrm{d}, J=6 \mathrm{~Hz}), 71.6(\mathrm{~d}, J=6 \mathrm{~Hz}), 41.2(\mathrm{~d}, J=4 \mathrm{~Hz}), 27.1(\mathrm{~s}), 22.7(\mathrm{~s}) .{ }^{31} \mathrm{P}\left\{{ }^{1} \mathrm{H}\right\} \mathrm{NMR}\left(121 \mathrm{MHz}, \mathrm{C}_{6} \mathrm{D}_{6}\right): \delta$ 40.7 (d, $J=24.2 \mathrm{~Hz}$ ), 17.0 (d, $J=24.2 \mathrm{~Hz}$ ). IR (nujol, NaCl): 2813 (s), 1624 (s), 1584 (s), 1479 (s), 1435 (s), 1287 (m), 1229 (m), 1181 (m), 1164 (s), 1095 (s), 1072 (w), 1035 (s), 999 (w), 940 (w), 880 (w), 827 (m), $764(\mathrm{~m}), 748(\mathrm{~s}), 683(\mathrm{~s}), 637(\mathrm{~m}), 543(\mathrm{~s}), 513(\mathrm{~s}), 493(\mathrm{~s}), 474(\mathrm{~m}), 459(\mathrm{~m}) \mathrm{cm}^{-1}$. Anal. Calcd. for $\mathrm{C}_{45} \mathrm{H}_{39} \mathrm{FFeNiOP}{ }_{2} \cdot 1 / 2 \mathrm{C}_{6} \mathrm{H}_{6}$ : C, 71.21; H, 5.49. Found: C, 71.11; H, 5.62.

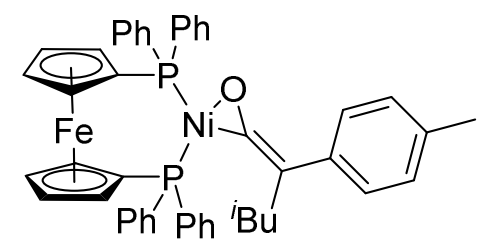

(Dppf)Ni(p-methylphenyl iso-butyl ketene), 8cc was synthesized according to the general procedure using $p$-methylphenyl isobutyl ketene 
(205.3 mg, $1.09 \mathrm{mmol}, 3$ equiv). The reaction was stirred for $5 \mathrm{~h}$, and pentane (15 mL) was added. After $16 \mathrm{~h}$, red needles had formed which were collected by filtration, washed with pentane $(3 \times 10 \mathrm{~mL})$ and dried under vacuum to yield the complex $(218.5 \mathrm{mg}, 75 \%)$. Crystals suitable for X-ray crystallography were grown by layering $1 \mathrm{~mL}$ of a saturated benzene solution of the complex with $0.5 \mathrm{~mL}$ benzene then $2.5 \mathrm{~mL}$ pentane. ${ }^{1} \mathrm{H}$ NMR $\left(400 \mathrm{MHz}, \mathrm{C}_{6} \mathrm{D}_{6}\right): \delta 8.16-7.97$ (br, $\left.10 \mathrm{H}\right), 7.20(\mathrm{~d}, J=8 \mathrm{~Hz}, 2 \mathrm{H}), 7.10$ (br, $\left.6 \mathrm{H}\right)$, $7.01(\mathrm{br}, 7 \mathrm{H}), 4.27$ (s with shoulder, $2 \mathrm{H}), 3.93(\mathrm{~s}, 1.82 \mathrm{H}), 3.87(\mathrm{~s}, 0.16 \mathrm{H}), 3.82(\mathrm{~s}, 0.14 \mathrm{H}), 3.74(\mathrm{~s}$, $1.89 \mathrm{H}), 3.66$ (s with shoulder, $2 \mathrm{H}), 2.22(\mathrm{~s}, 2.75 \mathrm{H}), 2.11-2.00(\mathrm{br}, 3 \mathrm{H}), 1.95(\mathrm{~s}, 0.20 \mathrm{H}), 1.17$ (d, $J=4$ $\mathrm{Hz}, 0.59 \mathrm{H}), 0.52(\mathrm{~d}, J=4 \mathrm{~Hz}, 5.07 \mathrm{H}) .{ }^{13} \mathrm{C}\left\{{ }^{1} \mathrm{H}\right\} \mathrm{NMR}\left(100.6 \mathrm{MHz}, \mathrm{C}_{6} \mathrm{D}_{6}\right): \delta 169.1(\mathrm{dd}, J=41,8 \mathrm{~Hz}$, $\mathrm{O}=C=\mathrm{C}), 138.6(\mathrm{~d}, J=8 \mathrm{~Hz}), 136.1(\mathrm{~d}, J=34 \mathrm{~Hz}), 135.7(\mathrm{~d}, J=15 \mathrm{~Hz}), 135.3(\mathrm{~d}, J=14 \mathrm{~Hz})$, this peak appears to overlap with a smaller peak, $134.9(\mathrm{~d}, J=2 \mathrm{~Hz}), 131.0(\mathrm{dd}, J=40,2 \mathrm{~Hz}), 131.1$ (s), $129.4(\mathrm{~d}$, $J=12 \mathrm{~Hz}), 129.3$ (d, $J=5 \mathrm{~Hz}), 129.2$ (s), 126.9 (s), 86.2 (dd, $J=48,4 \mathrm{~Hz}, \mathrm{O}=\mathrm{C}=C), 77.2$ (dd, $J=7,5$ $\mathrm{Hz}), 76.9(\mathrm{~d}, J=44 \mathrm{~Hz}), 75.3(\mathrm{~d}, J=11 \mathrm{~Hz}), 74.8(\mathrm{~d}, J=8 \mathrm{~Hz}), 73.4(\mathrm{~d}, J=6 \mathrm{~Hz}), 71.7(\mathrm{~d}, J=5 \mathrm{~Hz})$, $41.2(\mathrm{~d}, J=4 \mathrm{~Hz}), 27.1$ (s), 23.3 (s), 21.8 (s). ${ }^{31} \mathrm{P}\left\{{ }^{1} \mathrm{H}\right\} \mathrm{NMR}\left(121 \mathrm{MHz}, \mathrm{C}_{6} \mathrm{D}_{6}\right): \delta 40.3$ (d, $\left.J=23.0 \mathrm{~Hz}\right)$, 16.5 (d, $J=23.0 \mathrm{~Hz}$ ). IR (nujol, NaCl): 1959 (w), 1917 (w), 1768 (m), 1754 (m), 1625 (m), 1598 (s), 1508 (s), 1479 (s), 1435 (s), 1330 (w), 1308 (m), 1293 (m), 1284 (m), 1228 (w), 1193 (m), 1165 (s), 1095 (s), 1034 (s), 1000 (w), 883 (w), 820 (s). Anal. Calcd. for $\mathrm{C}_{47} \mathrm{H}_{44} \mathrm{FeNiOP}_{2}$ 1.5 $\mathrm{C}_{6} \mathrm{H}_{6}:$ C, 73.23; H, 5.82. Found: C, 72.95; H, 6.10.

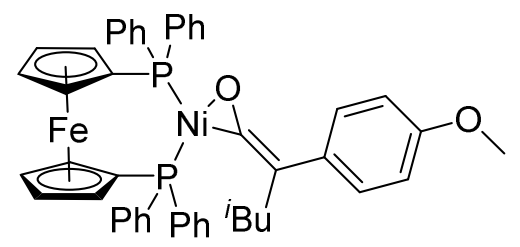

(Dppf)Ni(p-methoxyphenyl isobutyl ketene), 8cd was synthesized according to the general procedure using $p$-methoxyphenyl isobutyl ketene (222.6 mg, 3 equiv). The reaction was stirred for $4 \mathrm{~h}$ and pentane $(15 \mathrm{~mL})$ was added and the mixture was stored at $-40{ }^{\circ} \mathrm{C}$ for 16

$\mathrm{h}$. The solids were collected by filtration, washed $3 \times 10 \mathrm{~mL}$ pentane, dried under vacuum and collected by filtration to yield the complex as an orange solid (109.9 mg, 37\%). ${ }^{1} \mathrm{H}$ NMR $\left(300 \mathrm{MHz}, \mathrm{C}_{6} \mathrm{D}_{6}\right): \delta 8.40(\mathrm{t}, J$ $=10 \mathrm{~Hz}, 0.18 \mathrm{H}), 8.23(\mathrm{~d}, J=10 \mathrm{~Hz}, 0.20), 8.18(\mathrm{t}, J=10 \mathrm{~Hz}, 0.25 \mathrm{H}), 8.11(\mathrm{~m}, 3.21 \mathrm{H}), 8.02(\mathrm{~m}, 4.45 \mathrm{H})$, $7.77(\mathrm{~m}, 0.72 \mathrm{H}), 7.67(\mathrm{~m}, 0.09 \mathrm{H}), 7.42(\mathrm{t}, J=7.5 \mathrm{~Hz}, 0.08 \mathrm{H}), 7.35(\mathrm{br}, 0.24 \mathrm{H}), 7.31(\mathrm{~s}, 0.19 \mathrm{H}), 7.25(\mathrm{~m}$, $0.53 \mathrm{H}), 7.10(\mathrm{br}, 5.22 \mathrm{H}), 7.00(\mathrm{~m}, 7.66 \mathrm{H}), 6.94(\mathrm{~m}, 0.66 \mathrm{H}), 6.88(\mathrm{~d}, J=5 \mathrm{~Hz}, 0.34 \mathrm{H}), 6.82(\mathrm{~d}, J=5 \mathrm{~Hz}$, $0.32 \mathrm{H}), 4.28(\mathrm{~m}, 1.67 \mathrm{H}), 4.23(\mathrm{~s}, 0.13 \mathrm{H}), 4.16(\mathrm{~d}, J=10 \mathrm{~Hz}, 0.21 \mathrm{H}), 3.91(\mathrm{~s}, 1.67 \mathrm{H}), 3.85(\mathrm{~m}, 0.70 \mathrm{H})$, $3.80(\mathrm{~s}, 0.17 \mathrm{H}), 3.74(\mathrm{~m}, 1.69 \mathrm{H}), 3.70(\mathrm{~s}, 0.15 \mathrm{H}), 3.64(\mathrm{~m}, 1.62 \mathrm{H}), 3.38(\mathrm{~s}, 2.01 \mathrm{H}), 3.32(\mathrm{~s}, 0.31 \mathrm{H}), 3.21$ $(\mathrm{s}, 0.23 \mathrm{H}), 3.11(\mathrm{~m}, 0.45 \mathrm{H}), 2.08(\mathrm{~m}, 1.51 \mathrm{H}), 2.02(\mathrm{~m}, 0.76 \mathrm{H}), 1.86(\mathrm{~m}, 0.16 \mathrm{H}), 1.74(\mathrm{~m}, 0.31 \mathrm{H}), 1.19(\mathrm{~d}$, $J=5 \mathrm{~Hz}, 0.61 \mathrm{H}), 1.09$ (d, $J=5 \mathrm{~Hz}, 0.37 \mathrm{H}), 0.93(\mathrm{~d}, J=10 \mathrm{~Hz}, 0.36 \mathrm{H}), 0.80(\mathrm{~d}, J=5 \mathrm{~Hz}, 0.15 \mathrm{H}), 0.77$ $(\mathrm{d}, J=5 \mathrm{~Hz}, 0.30 \mathrm{H}), 0.63(\mathrm{~d}, J=5 \mathrm{~Hz}, 0.30 \mathrm{H}), 0.53(\mathrm{~d}, J=5 \mathrm{~Hz}, 4.27 \mathrm{H}) .{ }^{13} \mathrm{C}\left\{{ }^{1} \mathrm{H}\right\} \mathrm{NMR}(100.6 \mathrm{MHz}$, $\left.\mathrm{C}_{6} \mathrm{D}_{6}\right): \delta 168.8(\mathrm{dd}, J=40,4 \mathrm{~Hz}, \mathrm{O}=C=\mathrm{C}), 158.8(\mathrm{~d}, J=43 \mathrm{~Hz}), 156.24$ (s), $137.6(\mathrm{br}), 136.9(\mathrm{~d}, J=9$ $\mathrm{Hz}), 136.6(\mathrm{~d}, J=7 \mathrm{~Hz}), 136.2(\mathrm{~d}, J=34 \mathrm{~Hz}), 135.8(\mathrm{~d}, J=15 \mathrm{~Hz}), 135.5$ (d, $J=8 \mathrm{~Hz}), 135.3(\mathrm{~d}, J=13$ Hz), 135.0 (br) 134.8 (br), 134.4 (s), 134.2 (d, $J=8 \mathrm{~Hz}$ ), 133.7 (d, $J=12 \mathrm{~Hz}$ ), 131.0 (d, $J=42 \mathrm{~Hz}$ ), 
130.5 (d, $J=13 \mathrm{~Hz}), 130.0$ (s), 129.5 (s), 129.3 (d, $J=10 \mathrm{~Hz}), 129.2$ (dd, $J=9 \mathrm{~Hz}), 128.7$ (s), 128.5 (s) The last two peaks overlap with solvent, 127.9 (s), 127.8 (s), 115.4 (s), 114.5 (s), 114.2 (s), 114.1 (s), 113.9 (s), 86.2 (dd, $J=48,5 \mathrm{~Hz}), 77.6(\mathrm{~d}, J=37 \mathrm{~Hz}), 76.9$ (d, $J=44 \mathrm{~Hz}), 76.6(\mathrm{t}, J=7 \mathrm{~Hz}, \mathrm{O}=\mathrm{C}=C)$, $75.3(\mathrm{~d}, J=9 \mathrm{~Hz}), 75.0$ (d, $J=10 \mathrm{~Hz}), 74.8$ (d, $J=8 \mathrm{~Hz}), 73.4$ (d, $J=5 \mathrm{~Hz}), 71.7$ (d, $J=4 \mathrm{~Hz}), 55.4$ (s), 55.3 (s), 55.25 (s), 55.21 (s), 50.7 (s), 48.5 (s), 41.3 (s), 27.1 (s), 26.4 (s), 25.9 (d, J = 4 Hz), 25.5 (s), 24.1 (s), 23.8 (s), 23.7 (s), 23.3 (s). ${ }^{31} \mathrm{P}\left\{{ }^{1} \mathrm{H}\right\}$ NMR (121 MHz, $\left.\mathrm{C}_{6} \mathrm{D}_{6}\right): \delta 40.3$ (d, $\left.J=22 \mathrm{~Hz}\right), 33.0$ (d, $J=30$ Hz), 23.0 (s), 21.7 (d, $J=30 \mathrm{~Hz}), 16.5$ (d, $J=23 \mathrm{~Hz}$ ). IR (nujol, NaCl): 1915 (w), 1752 (w), 1626 (m), 1506 (s), 1436 (s), 1280 (m), 1244 (s), 1179 (m), 1095 (m), 1036 (m), 830 (m), 746 (s), 697 (s), 637 (m). Anal. Calcd. for $\mathrm{C}_{47} \mathrm{H}_{44} \mathrm{FeNiO}_{2} \mathrm{P}_{2}$ : C, 69.07; H, 5.43. Found: C, 69.21; H,

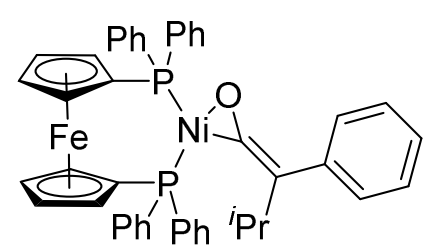
5.40 .

(Dppf)Ni(phenyl isopropyl ketene), 8d was synthesized according to the general procedure with phenyl isopropyl ketene $(174.7 \mathrm{mg}, 1.09 \mathrm{mmol}, 3$ equiv). The reaction was stirred for $48 \mathrm{~h}$, and $15 \mathrm{~mL}$ pentane was added. After an additional $24 \mathrm{~h}$, the solids were collected by filtration, washed with $3 \times 10 \mathrm{~mL}$ pentane, and dried under vacuum to afford $224.9 \mathrm{mg}, 80 \%$ of a yellow powder. ${ }^{1} \mathrm{H}$ NMR $\left(300 \mathrm{MHz}, \mathrm{C}_{6} \mathrm{D}_{6}\right): \delta 8.27(\mathrm{~d}, J=8$ $\mathrm{Hz}, 1 \mathrm{H}), 8.06(\mathrm{t}, J=8 \mathrm{~Hz}, 3 \mathrm{H}), 7.99(\mathrm{t}, J=8 \mathrm{~Hz}, 3 \mathrm{H}), 7.90(\mathrm{dd}, J=8,4 \mathrm{~Hz}, 1 \mathrm{H}), 7.71(\mathrm{~s}, \mathrm{br}, 1 \mathrm{H}), 7.37$ (t, $J=8 \mathrm{~Hz}, 2 \mathrm{H}), 7.09$ (m, br, 5H), 7.00 (m, br, 9H), 6.89 (m, br, 0.77H), 6.81 (t, $J=8 \mathrm{~Hz}, 0.39 \mathrm{H}), 6.67$ (d, $J=8 \mathrm{~Hz}, 0.48 \mathrm{H}), 6.57(\mathrm{~m}, 0.46 \mathrm{H}), 4.28(\mathrm{~s}, 1.35 \mathrm{H}), 4.25(\mathrm{~s}, 0.46 \mathrm{H}), 3.91(\mathrm{~s}, 1.42 \mathrm{H}), 3.87(0.51 \mathrm{H}), 3.83$ $(0.56 \mathrm{H}), 3.78(\mathrm{~s}, 0.60 \mathrm{H}), 3.75(\mathrm{~s}, 0.63 \mathrm{H}), 3.72(\mathrm{~s}, 1.45 \mathrm{H}), 3.65(1.63 \mathrm{H}), 3.34$ (quint, $J=8 \mathrm{~Hz}, 0.14 \mathrm{H})$, 2.57 (quint, $J=8 \mathrm{~Hz}, 0.62 \mathrm{H}$ ), 2.35 (quint, $J=8 \mathrm{~Hz}, 0.24 \mathrm{H}$ ), 1.67 (d, $J=4 \mathrm{~Hz}, 0.92 \mathrm{H}), 1.32(\mathrm{~d}, J=8 \mathrm{~Hz}$, $0.89 \mathrm{H}), 1.16(\mathrm{~d}, J=8 \mathrm{~Hz}, 0.82 \mathrm{H}),, 1.00(\mathrm{~d}, J=4 \mathrm{~Hz}, 3.86 \mathrm{H}) .{ }^{13} \mathrm{C}\left\{{ }^{1} \mathrm{H}\right\}$ NMR $\left(125 \mathrm{MHz}, \mathrm{THF}-d_{8}\right): \delta 168.6$ $(\mathrm{dd}, J=41,9 \mathrm{~Hz}, \mathrm{O}=C=\mathrm{C}), 140.9(\mathrm{~d}, J=8 \mathrm{~Hz}), 136.7(\mathrm{~d}, J=34 \mathrm{~Hz}), 136.3(\mathrm{~d}, J=14 \mathrm{~Hz}), 136.7(\mathrm{~d}, J=$ $13 \mathrm{~Hz}), 136.6$ (dd, $J=41,3 \mathrm{~Hz}), 131.9$ (d, $J=2 \mathrm{~Hz}), 131.3$ (d, $J=2 \mathrm{~Hz}), 129.9$ (d, $J=10 \mathrm{~Hz}), 129.6$ (d, $J=10 \mathrm{~Hz}), 128.6$ (s), 126.0 (s), 121.8 (s), 86.0 (dd, $J=49,6 \mathrm{~Hz}), 79.2(\mathrm{dd}, J=8,5 \mathrm{~Hz}, \mathrm{O}=\mathrm{C}=C), 76.9$ $(\mathrm{d}, J=45 \mathrm{~Hz}), 75.9$ (d, $J=11 \mathrm{~Hz}), 75.4$ (d, $J=8 \mathrm{~Hz}), 75.2$ (d, $J=6 \mathrm{~Hz}), 72.5$ (d, $J=6 \mathrm{~Hz}), 35.0(\mathrm{~d}, J=$ $4 \mathrm{~Hz}), 24.0(\mathrm{~s}), 14.8(\mathrm{~s}) .{ }^{31} \mathrm{P}\left\{{ }^{1} \mathrm{H}\right\} \operatorname{NMR}\left(121 \mathrm{MHz}, \mathrm{C}_{6} \mathrm{D}_{6}\right): \delta 45.9(\mathrm{~d}, J=24.2 \mathrm{~Hz}), 45.2(\mathrm{~d}, J=24.2 \mathrm{~Hz})$, 43.4 (d, $J=24.2 \mathrm{~Hz}$ ), 26.94 (s), 20.6 (d, $J=24.2 \mathrm{~Hz}$ ). IR (nujol, NaCl): 3105 (m), 3090 (m), 2361 (w), 1915 (w), 1611 (s), 1582 (s), 1491 (s), 1479 (s), 1436 (s), 1381 (m), 1306 (m), 1271 (m), 1196 (m), 1158 (m), 1096 (s), 1070 (w), 1029 (m), 998 (m), 976 (m), 897 (w), 865 (w), 840 (w), 825 (m), 812 (w) cm ${ }^{-1}$. Anal. Calcd. for $\mathrm{C}_{45} \mathrm{H}_{40} \mathrm{FeNiOP}_{2}$ : C, 69.89; H, 5.21. Found: C, 70.08; H, 5.30.

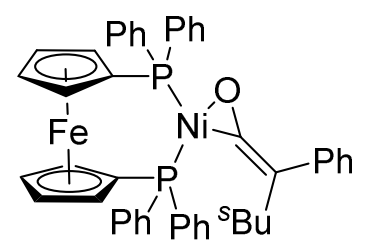

(Dppf)Ni(phenyl sec-butyl ketene), 8e was synthesized by adding a solution of phenyl sec-butyl ketene (316.5 mg, $1.82 \mathrm{mmol}, 5$ equiv) in benzene ( $2 \mathrm{~mL})$ to a suspension of (dppf)Ni(COD) (261.5 mg, $0.363 \mathrm{mmol}, 1$ equiv) in benzene ( 3 
$\mathrm{mL})$. After $7 \mathrm{~h}$, the reaction had turned from an orange suspension to a red solution, and pentane (15 $\mathrm{mL})$ was added. After an additional $16 \mathrm{~h}$, the solids were collected by filtration, washed $3 \mathrm{x} 10 \mathrm{~mL}$ pentane, and dried under vacuum. The complex is a red crystalline solid (223.3 mg, 78\%). Crystals suitable for x-ray diffraction were by layering $1 \mathrm{~mL}$ of a saturated solution of the complex in benzene with $0.5 \mathrm{~mL}$ benzene followed by $3.5 \mathrm{~mL}$ pentane. ${ }^{1} \mathrm{H}$ NMR $\left(500 \mathrm{MHz}, \mathrm{C}_{6} \mathrm{D}_{6}\right)$ : $\delta$ Aromatic region: $8.24(\mathrm{~m}, 1.16 \mathrm{H}), 8.17-8.03$ $(\mathrm{m}, 3.07 \mathrm{H}), 8.03-7.94(\mathrm{q}, J=10 \mathrm{~Hz}, 2.38 \mathrm{H}), 7.93-7.87$ (br, 0.94H), 7.76-7.66 (br, 0.83H), 7.42 (t, $J=7.5$ $\mathrm{Hz}, 0.42 \mathrm{H}), 7.37$ (t, $J=7.5 \mathrm{~Hz}, 0.90 \mathrm{H}), 7.22(\mathrm{~d}, J=5 \mathrm{~Hz}, 0.56 \mathrm{H}), 7.15-6.91(\mathrm{~m}, 12.80 \mathrm{H}), 6.88(\mathrm{t}, J=7.5$ $\mathrm{Hz}, 0.47 \mathrm{H}), 6.81(\mathrm{t}, J=5 \mathrm{~Hz}, 0.26 \mathrm{H}), 6.69(\mathrm{~d}, J=10 \mathrm{~Hz}, 0.20 \mathrm{H}), 6.65(\mathrm{~d}, J=10 \mathrm{~Hz}, 0.27 \mathrm{H}), 6.60(\mathrm{~m}$, $0.24 \mathrm{H}), 6.54(\mathrm{t}, J=7.5 \mathrm{~Hz}, 0.39 \mathrm{H}), \mathrm{Cp}$ region: $4.74(\mathrm{~s}, 0.07 \mathrm{H}), 4.68-4.53(\mathrm{br}, 0.40 \mathrm{H}), 4.48(\mathrm{br}, 0.11 \mathrm{H})$, $4.40(\mathrm{~s}, 0.36 \mathrm{H}), 4.36(\mathrm{~s}, 0.05 \mathrm{H}), 4.30-4.24(\mathrm{br}, 0.54 \mathrm{H}), 4.22(\mathrm{~s}, 0.17 \mathrm{H}), 4.12(\mathrm{~s}, 0.36 \mathrm{H}), 4.06(\mathrm{~s}, 0.05 \mathrm{H})$, $3.39(\mathrm{~s}, 0.46 \mathrm{H}), 3.90(\mathrm{~s}, 0.37 \mathrm{H}), 3.88-3.85(\mathrm{~m}, 0.86 \mathrm{H}), 3.84-3.79(\mathrm{~m}, 0.99 \mathrm{H}), 3.77(\mathrm{~s}, 0.48 \mathrm{H}), 3.74(\mathrm{~m}$, $078 \mathrm{H}), 3.65(\mathrm{~m}, 1.83 \mathrm{H}), 3.02(\mathrm{q}, J=5 \mathrm{~Hz}, 0.19 \mathrm{H})$, Alkyl region: $2.30(\mathrm{~m}, 0.23 \mathrm{H}), 2.25-2.11(\mathrm{~m}, 0.89 \mathrm{H})$, $1.89-1.76(\mathrm{~m}, 0.71 \mathrm{H}), 1.69-1.59(\mathrm{~m}, 1.15 \mathrm{H}), 1.31(\mathrm{~d}, J=5 \mathrm{~Hz}, 0.63 \mathrm{H}), 1.25(\mathrm{t}, J=7.5 \mathrm{~Hz}, 0.92 \mathrm{H}), 1.12$ $(\mathrm{d}, J=5 \mathrm{~Hz}, 0.28 \mathrm{H}), 0.91(\mathrm{~d}, J=0.5 \mathrm{~Hz}, 1.19 \mathrm{H}), 0.86(\mathrm{t}, J=7.5 \mathrm{~Hz}, 0.85 \mathrm{H}), 0.76(\mathrm{~m}, 0.37 \mathrm{H}), 0.58(\mathrm{~s}$, $0.65 \mathrm{H}), 0.26(\mathrm{t}, J=5 \mathrm{~Hz}, 1.18 \mathrm{H}) .{ }^{13} \mathrm{C}\left\{{ }^{1} \mathrm{H}\right\} \mathrm{NMR}\left(125 \mathrm{MHz}, \mathrm{C}_{6} \mathrm{D}_{6}\right): \delta 233.6(\mathrm{dd}, J=84,23 \mathrm{~Hz}), 168.7$ $(\mathrm{dd}, J=40,9 \mathrm{~Hz}), 168.5$ (dd, $J=40,8 \mathrm{~Hz}), 163.2(\mathrm{dd}, J=41,8 \mathrm{~Hz}), 145.5(\mathrm{~d}, J=2 \mathrm{~Hz}), 141.0(\mathrm{~d}, J=8$ $\mathrm{Hz}), 140.7$ (d, $J=8 \mathrm{~Hz}), 140.3(\mathrm{dd}, J=31,4 \mathrm{~Hz}), 136.45(\mathrm{dd}, J=32,18 \mathrm{~Hz}), 136.36(\mathrm{~d}, J=1 \mathrm{~Hz})$, 136.14 (s), 136.1 (d, $J=14 \mathrm{~Hz}), 135.8$ (d, $J=5 \mathrm{~Hz}), 135.7$ (d, $J=14 \mathrm{~Hz}), 135.6$ (d, $J=14 \mathrm{~Hz}), 135.5$ (s), $135.4(\mathrm{~d}, J=6 \mathrm{~Hz}), 135.3$ (d, $J=2 \mathrm{~Hz}), 135.20$ (s), 135.19 (d, $J=15 \mathrm{~Hz}), 134.94$ (dd, $J=25,2 \mathrm{~Hz})$, 134.92 (d, $J=12 \mathrm{~Hz}), 134.7$ (t, $J=2 \mathrm{~Hz}), 134.3$ (t, $J=2 \mathrm{~Hz}), 132.5$ (br), 131.22 (dd, $J=46,1 \mathrm{~Hz}$ ), $131.22(\mathrm{~d}, J=2 \mathrm{~Hz}), 130.9$ (d, $J=1 \mathrm{~Hz}), 130.8$ (dd, $J=31,1 \mathrm{~Hz}), 130.76$ (br), 129.24-129.12 (m, possibly two dd), 129.0 (s, overlaps with solvent), 128.9 (s, overlaps with solvent, possible multiplet with previous peak), $128.2(\mathrm{~s}), 128.0(\mathrm{~s}), 126.2(\mathrm{~s}), 123.4(\mathrm{~s}), 123.0(\mathrm{~s}), 122.8(\mathrm{~s}), 122.6(\mathrm{~s}), 122.4(\mathrm{~s}), 122.2(\mathrm{~d}$, $J=6 \mathrm{~Hz}), 86.3(\mathrm{~d}, J=6 \mathrm{~Hz}), 86.2(\mathrm{~d}, J=6 \mathrm{~Hz}), 86.0(\mathrm{dd}, J=6,5 \mathrm{~Hz}), 85.9(\mathrm{dd}, J=6,4 \mathrm{~Hz}), 85.8(\mathrm{~d}, J$ $=5 \mathrm{~Hz}), 85.5(\mathrm{~d}, J=5 \mathrm{~Hz}), 84.7(\mathrm{dd}, J=8,5 \mathrm{~Hz}), 79.3(\mathrm{dd}, J=6,5 \mathrm{~Hz}), 77.0(\mathrm{~d}, J=44 \mathrm{~Hz}), 76.9(\mathrm{~d}, J$ $=44 \mathrm{~Hz}), 76.62(\mathrm{t}, J=16 \mathrm{~Hz}), 76.2(\mathrm{~d}, J=44 \mathrm{~Hz}), 75.3(\mathrm{t}, J=14 \mathrm{~Hz}), 75.2(\mathrm{dd}, J=11,4 \mathrm{~Hz}), 75.0(\mathrm{~d}, J$ $=5 \mathrm{~Hz}), 74.8(\mathrm{~d}, J=9 \mathrm{~Hz}), 74.7(\mathrm{t}, J=9 \mathrm{~Hz}), 74.5(\mathrm{~d}, J=5 \mathrm{~Hz}), 74.4(\mathrm{~d}, J=10 \mathrm{~Hz}), 73.9(\mathrm{~d}, J=8 \mathrm{~Hz})$, $73.5(\mathrm{~d}, J=6 \mathrm{~Hz}), 73.3(\mathrm{dd}, J=22,6 \mathrm{~Hz}), 72.8(\mathrm{~d}, J=6 \mathrm{~Hz}), 72.1(\mathrm{~d}, J=5 \mathrm{~Hz}), 72.0(\mathrm{dd}, J=26,6 \mathrm{~Hz})$, $71.7(\mathrm{~d}, J=5 \mathrm{~Hz}), 71.2$ (d, $J=5 \mathrm{~Hz}), 42.0$ (d, $J=6 \mathrm{~Hz}), 40.1$ (d, $J=4 \mathrm{~Hz}), 33.1(\mathrm{~s}), 32.8(\mathrm{~m}), 31.9$ (t, $J$ $=4 \mathrm{~Hz}), 30.6(\mathrm{~s}), 30.4(\mathrm{~s}), 30.0(\mathrm{~s}), 27.5(\mathrm{~s}), 23.9(\mathrm{~s}), 23.3(\mathrm{~s}), 21.5(\mathrm{~s}), 20.3(\mathrm{~s}), 20.2(\mathrm{~s}), 16.7(\mathrm{~s}), 14.9(\mathrm{~s})$, 14.2 (s), 13.9 (s), 13.3 (s), 12.3. ${ }^{31} \mathrm{P}\left\{{ }^{1} \mathrm{H}\right\}$ NMR (121 MHz, $\left.\mathrm{C}_{6} \mathrm{D}_{6}\right): \delta 43.6(\mathrm{~d}, J=23 \mathrm{~Hz}), 42.9(\mathrm{~d}, J=24$ $\mathrm{Hz}), 41.0(\mathrm{~d}, J=24 \mathrm{~Hz}), 24.7$ (s), 18.8 (d, $J=23 \mathrm{~Hz}), 18.3$ (d, $J=24 \mathrm{~Hz}), 18.1$ (d, $J=24 \mathrm{~Hz})$. IR (nujol, $\mathrm{NaCl}$ ): 2091 (w), 1913 (s), 1727 (w), 1614 (m), 1581 (s), 1435 (s), 1306 (m), 1265 (m), 1160 (m), 1095 
(s), $1071(w), 1028$ (s), 998 (m), 894 (w), 821 (m), 748 (s), 695 (s), 678 (s), 637 (w). Anal. Calcd. for $\mathrm{C}_{46} \mathrm{H}_{42} \mathrm{FeNiOP}_{2}$ : C, 70.17; H, 5.38. Found: C, 69.93; H, 5.43.

Kinetic Analyses (Dppf)Ni(ketene) complex $(0.01 \mathrm{mmol})$ and 1,3,5-trimethoxybenzene $(0.008 \mathrm{mmol})$ were weighed into a 4-mL scintillation vial in the glovebox and dissolved in toluene- $d_{8}(0.75 \mathrm{~mL})$. The samples were transferred through a pipet filter with glass wool to screw cap NMR tubes and analyzed by ${ }^{1} \mathrm{H}$ NMR $(500 \mathrm{MHz})$. The tubes were immersed in a $100{ }^{\circ} \mathrm{C}$ oil bath for the indicated amount of time, cooled to room temperature, and analyzed again by ${ }^{1} \mathrm{H}$ NMR. The heating/NMR cycles were repeated until 25-30 time points had been obtained or the starting material was no longer observed. Two or three kinetic data sets were collected for each complex. In several cases, the $\mathrm{T}_{0}$ did not fit linearly in the $\ln \left([\mathrm{A}] /[\mathrm{A}]_{0}\right)$ vs time plot with the rest of the data. In these cases, the reaction appeared to have a significantly faster rate over the first time point, due to population of the $\mathrm{CC}$ coordination mode. The $\mathrm{T}_{0}$ was omitted and the $T_{1}$ treated as the $T_{0}$. In some trials, significant line broadening was observed after a few time points. We believe this is due to oxygen permeating into the NMR tube, and these trials were excluded. Rate constants and other data associated with each trial in Table S1.

Table S1. Data for kinetics of decomposition of (dppf)Ni(ketene) complexes.

\begin{tabular}{|c|c|c|c|c|c|c|}
\hline Complex & $\mathrm{K}_{\mathrm{obs}}\left(\times 10^{-4} / \mathrm{s}\right)$ & $\mathrm{R}^{2}$ & Timepoints included & Time between points ( $\mathrm{min}$ ) & average $\mathrm{K}_{\mathrm{obs}}$ & st. dev. \\
\hline \multirow[t]{2}{*}{$8 b$} & 1.038 & 0.9984 & $1-25$ & 10 & \multirow[t]{2}{*}{1.038} & \multirow[t]{2}{*}{0.001} \\
\hline & 1.037 & 0.9974 & $0-30$ & 10 & & \\
\hline \multirow[t]{3}{*}{$8 b-d 9$} & 0.7855 & 0.9971 & $1-30$ & 10 & \multirow[t]{3}{*}{0.7709} & \multirow[t]{3}{*}{0.0156} \\
\hline & 0.7726 & 0.9986 & $0-30$ & 15 & & \\
\hline & 0.7545 & 0.9919 & $0-25$ & 15 & & \\
\hline \multirow[t]{2}{*}{$8 \mathrm{ca}$} & 0.9396 & 0.9941 & $1-23$ & 10 & \multirow[t]{2}{*}{0.9971} & \multirow[t]{2}{*}{0.0814} \\
\hline & 1.055 & 0.9979 & $0-25$ & 15 & & \\
\hline \multirow[t]{3}{*}{$8 c b$} & 4.745 & 0.9925 & $1-23$ & 5 & \multirow[t]{3}{*}{5.020} & \multirow[t]{3}{*}{0.263} \\
\hline & 5.270 & 0.9968 & $1-17$ & 5 & & \\
\hline & 5.046 & 0.9937 & $1-15$ & 5 & & \\
\hline \multirow[t]{3}{*}{$8 c c$} & 3.215 & 0.994 & $1-27$ & 5 & \multirow[t]{3}{*}{3.570} & \multirow[t]{3}{*}{0.326} \\
\hline & 3.637 & 0.9959 & $1-27$ & 5 & & \\
\hline & 3.857 & 0.9956 & $1-26$ & 5 & & \\
\hline \multirow[t]{3}{*}{$8 \mathrm{~cd}$} & 2.924 & 0.9959 & $1-25$ & 5 & \multirow[t]{3}{*}{2.692} & \multirow[t]{3}{*}{0.267} \\
\hline & 2.401 & 0.9934 & $0-29$ & 5 & & \\
\hline & 2.750 & 0.9981 & $1-29$ & 5 & & \\
\hline \multirow[t]{2}{*}{$8 d$} & 2.467 & 0.9958 & $1-23$ & 5 & \multirow[t]{2}{*}{2.406} & \multirow[t]{2}{*}{0.086} \\
\hline & 2.345 & 0.9923 & $1-30$ & 5 & & \\
\hline
\end{tabular}

\section{Computational Details}

All calculations were performed with the GAUSSIAN09 ${ }^{7}$ suite of programs. The structure of (Dppf)Ni(Phenyl isobutyl ketene), 8cb, was optimized with the $\mathrm{B}^{2} \mathrm{LYP}^{8}$ functional and a split basis set 
consisting of tzvp for the $\mathrm{P}, \mathrm{Ni}$, and ketene $\mathrm{O}=\mathrm{C}=\mathrm{C}$ atoms and svp for all other atoms. ${ }^{9}$ The structure was also optimized with BP86 $6^{10}$ and the same split basis set and at the B3LYP/tzvp and BP86/tzvp levels of theory. Solvent (benzene) effects were taken into account using the self consistent reaction field (SCRF) with the PCM model. Analytical frequency calculations at the same level of theory were performed to ensure the calculated structures are local minima and to generate thermal correction factors to ZPE, energy, enthalpy, and gibbs free energy. Several important bond lengths and angles were compared from the crystal structure of this complex and the calculated structures (Table S2). The average of the differences between calculated structures and the crystal structure reveal that BP 86 reproduces the crystal structure with greater accuracy than B3LYP. In particular, B3LYP overestimates the Ni-P bond lengths. Furthermore, there is no significant difference between the computationally more expensive tzvp basis set and the split tzvp/svp basis set. Further optimizations and frequency calculations were therefore done with the BP86 functional and the split tzvp/svp basis set. Population ${ }^{11}$ calculations were performed at the same level of theory and analyzed with QMForge. ${ }^{12}$ Transition states were located by performing 1dimensional potential energy scans, running a frequency calculation on the highest point from the PES, and using geometries along the negative frequency as input structures for the QST2 method. Frequency calculations were done on the transition structures to ensure they are first order saddle points. Intrinsic reaction coordinate (IRC) calculations were done in most cases to ensure the transition state connects with the desired intermediates, except when the negative frequency clearly describes the desired reaction path such as in the case of phosphine dissociation. IRCs were also not performed if a transition state closely resembled a transition state on which an IRC had been done, i.e. no IRCs were done on TS3-trans and TS2a, because they resemble TS3-cis and TS2, respectively. 
Table S2. Calculated and experimental bond angles and lengths for $\mathbf{8 c b}$.

\begin{tabular}{|c|c|c|c|c|c|c|c|c|c|}
\hline \multirow[b]{2}{*}{ Bonds } & \multirow[b]{2}{*}{ Experimental } & \multicolumn{2}{|c|}{ B3LYP/split } & \multicolumn{2}{|c|}{ BP86/split } & \multicolumn{2}{|c|}{ BP86/tzvp } & \multicolumn{2}{|c|}{ B3LYP/tzvp } \\
\hline & & Length & |difference| & Length & |difference| & Length & |difference| & Length & |difference| \\
\hline $\mathrm{P}_{1} \mathrm{Ni}$ & 2.231 & 2.316 & 0.085 & 2.260 & 0.029 & 2.264 & 0.033 & 2.322 & 0.091 \\
\hline $\mathrm{P}_{2} \mathrm{Ni}$ & 2.157 & 2.216 & 0.059 & 2.174 & 0.017 & 2.178 & 0.021 & 2.221 & 0.064 \\
\hline $\mathrm{ONi}$ & 1.855 & 1.871 & 0.016 & 1.893 & 0.038 & 1.896 & 0.041 & 1.874 & 0.019 \\
\hline $\mathrm{CNi}$ & 1.886 & 1.898 & 0.012 & 1.905 & 0.019 & 1.909 & 0.023 & 1.901 & 0.015 \\
\hline $\mathrm{CO}$ & 1.292 & 1.280 & 0.012 & 1.289 & 0.004 & 1.287 & 0.005 & 1.278 & 0.014 \\
\hline \multirow[t]{2}{*}{ CC } & 1.354 & 1.355 & 0.001 & 1.365 & 0.011 & 1.365 & 0.011 & 1.354 & 0.000 \\
\hline & & avg & \multicolumn{2}{|c|}{0.031 avg } & \multicolumn{2}{|c|}{0.020 avg } & \multicolumn{2}{|c|}{0.022 avg } & 0.034 \\
\hline Angles & Experimental $\left({ }^{\circ}\right)$ & Angle $\left({ }^{\circ}\right)$ & |difference| & Angle $\left({ }^{\circ}\right)$ & |difference| & Angle $\left({ }^{\circ}\right)$ & |difference| & Angle $\left({ }^{\circ}\right)$ & |difference| \\
\hline $\mathrm{O} \mathrm{NiP} \mathrm{P}_{1}$ & 100.98 & 97.34 & 3.64 & 98.62 & 2.36 & 98.92 & 2.06 & 97.56 & 3.42 \\
\hline $\mathrm{P}_{1} \mathrm{Ni}_{2}$ & 104.78 & 104.71 & 0.07 & 104.26 & 0.52 & 104.38 & 0.40 & 104.84 & 0.06 \\
\hline $\mathrm{P}_{2} \mathrm{NiC}_{1}$ & 113.54 & 118.77 & 5.23 & 118.08 & 4.54 & 117.91 & 4.37 & 118.67 & 5.13 \\
\hline $\mathrm{CNiO}$ & 40.41 & 39.69 & 0.72 & 39.66 & 0.75 & 39.55 & 0.86 & 39.58 & 0.83 \\
\hline $\mathrm{NiOC}$ & 71.07 & 71.27 & 0.20 & 70.68 & 0.39 & 70.80 & 0.27 & 71.37 & 0.30 \\
\hline $\mathrm{NiCO}$ & 68.51 & 69.04 & 0.53 & 69.66 & 1.15 & 69.65 & 1.14 & 69.05 & 0.54 \\
\hline \multirow[t]{2}{*}{ OCC } & 135.18 & 135.12 & 1.73 & 135.54 & 1.62 & 135.79 & 1.52 & 135.40 & 1.71 \\
\hline & & avg & 1.32 & avg & 1.27 & ivg & 1.23 & avg & 1.31 \\
\hline
\end{tabular}

Several levels of theory were evaluated for single point calculations (Table S3). Two factors were taken into account for each level of theory: The CC coordinated complex 9 must be higher in energy than the CO coordinated $8 \mathrm{~b}$, and the barrier for decarbonylation (the energy of TS2 the energy of 9) must be close to the experimentally derived $28.8 \mathrm{kcal} / \mathrm{mol}$. Most levels of theory examined predicted that the CC coordination would is preferred and that the barrier for decarbonylation is much higher than the experimental value (Table SI-2). For the split basis sets, SDD $^{13}$ is used for Ni and the other basis for all other atoms. At a first glance, the M06 ${ }^{14}$ functional combined with SDD for nickel and 6-311+G(2d,p) for all other atoms, which was used by our collaborators in a mechanistic analysis of the Ni catalyzed $[2+2$ +2 ] cycloaddition of diynes and tropone performed the best. ${ }^{15}$ However, the energy of carbonyl carbene complex $\mathbf{1 0}$ at this level of theory is $50.4 \mathrm{kcal} / \mathrm{mol}$ relative to $\mathbf{8 b}$. We suspect the disparity of applicability of this level of theory for single point energy calculations to the diyne/tropone system vs the ketene system is because the large SDD basis set was employed for Ni in the optimization and frequency calculations for the tropone/diyne system and not the ketene system. To our surprise (and possibly the chagrin of theoretical chemists) the relatively low level of theory BLYP/6-31g* provided energies of 9 and TS2 relative to $\mathbf{8 b}$ compatible with our experimental observations. This level of theory was chosen for the rest of the single point energy calculations. 
Table S3. Energies of $\mathbf{9}$ and TS2 relative to $8 \mathbf{b}$ at different levels of theory.

\begin{tabular}{|l|l|r|r|}
\cline { 3 - 4 } & & \multicolumn{2}{|c|}{ Rree energy (kcal/mol) } \\
\hline Func & Basis & TS2 & \multicolumn{1}{|c|}{9} \\
\hline BP86 & $6-311 \mathrm{G}+(2 \mathrm{~d}, \mathrm{p})$ & 33.5 & 0.7 \\
\hline M06 & SDD/6-311G+(2d,p) & 30.4 & 0.4 \\
\hline M062x & SDD/6-311G+(2d,p) & 34.1 & 4.0 \\
\hline M06I & tzvp & 30.6 & -5.5 \\
\hline m06l & $6-311 \mathrm{~g}^{*}$ & 26.6 & -8.6 \\
\hline PBE & tzvp & 32.5 & -2.0 \\
\hline PBE & $6-311 \mathrm{~g}^{*}$ & 29.2 & -4.2 \\
\hline M06l & $6-311 \mathrm{G}+(2 \mathrm{~d}, \mathrm{p})$ & 30.6 & -5.6 \\
\hline M06I & SDD/6-311G+(2d,p) & 27.9 & -1.3 \\
\hline PBE & $6-311 \mathrm{G}+(2 \mathrm{~d}, \mathrm{p})$ & 33.4 & -0.9 \\
\hline PBE & SDD/6-311G+(2d,p) & 31.9 & -0.4 \\
\hline M06l & SDD/6-311G* & 30.1 & -1.1 \\
\hline BP86 & $6-311 \mathrm{~g}^{*}$ & 29.2 & -2.4 \\
\hline B3LYP & $6-311 \mathrm{~g}^{*}$ & 38.3 & 3.9 \\
\hline M06l & $6-31 \mathrm{~g}^{*}$ & 24.6 & -7.0 \\
\hline BP86 & $6-31 \mathrm{~g}^{*}$ & 27.0 & -1.5 \\
\hline PBE & $6-31 \mathrm{~g}^{*}$ & 27.0 & -3.0 \\
\hline M06 & $6-31 \mathrm{~g}^{*}$ & 29.0 & -5.4 \\
\hline BLYP & $6-31 \mathrm{~g}^{*}$ & 28.7 & 1.9 \\
\hline M062x & $6-31 \mathrm{~g}^{*}$ & 40.8 & 5.0 \\
\hline
\end{tabular}

8cb - B3LYP/tzvp-svp

Coordinates:

$\begin{array}{lccc}\mathrm{C} & 3.79703600 & -0.26085500 & 0.39176500 \\ \mathrm{C} & 4.91798100 & -0.76606700 & -0.33116700 \\ \mathrm{C} & 4.92487500 & -0.15811000 & -1.62267400 \\ \mathrm{C} & 3.81492800 & 0.73450900 & -1.69917300 \\ \mathrm{C} & 3.10396400 & 0.67923600 & -0.45142500 \\ \mathrm{H} & 3.50996500 & -0.54409900 & 1.40237800 \\ \mathrm{H} & 5.62756700 & -1.50779500 & 0.03304400 \\ \mathrm{H} & 5.64119300 & -0.35332100 & -2.41962000 \\ \mathrm{H} & 3.53726200 & 1.33864800 & -2.56010900 \\ \mathrm{Fe} & 3.13777100 & -1.19277600 & -1.32481400 \\ \mathrm{C} & 1.37664000 & -1.61158500 & -2.31679000 \\ \mathrm{C} & 2.46844000 & -2.16388000 & -3.04807500 \\ \mathrm{C} & 1.37047100 & -2.19422300 & -0.99881500 \\ \mathrm{H} & 0.67468800 & -0.86337700 & -2.68100900 \\ \mathrm{C} & 3.14661000 & -3.08889300 & -2.19834400 \\ \mathrm{H} & 2.74998200 & -1.90352900 & -4.06742300 \\ \mathrm{C} & 2.47581200 & -3.11360900 & -0.94014300 \\ \mathrm{H} & 4.04025500 & -3.65700700 & -2.45382600 \\ \mathrm{H} & 2.77474800 & -3.70177900 & -0.07591000 \\ \mathrm{P} & 1.52887600 & 1.52485900 & -0.03612900 \\ \mathrm{P} & 0.12146000 & -1.76240300 & 0.27349200 \\ \mathrm{Ni} & -0.46727000 & 0.37265500 & 0.19120800\end{array}$




\begin{tabular}{|c|c|c|c|}
\hline $\mathrm{O}$ & -1.58917500 & 1.86374000 & 0.33192400 \\
\hline $\mathrm{C}$ & -2.31271200 & 0.81331900 & 0.22773200 \\
\hline $\mathrm{C}$ & -3.64387800 & 0.56381400 & 0.19727900 \\
\hline $\mathrm{C}$ & -4.62158300 & 1.65488100 & 0.41076200 \\
\hline $\mathrm{C}$ & -5.98116000 & 1.36569400 & 0.68624900 \\
\hline $\mathrm{C}$ & -4.26517000 & 3.02614200 & 0.37330400 \\
\hline $\mathrm{C}$ & -6.91775300 & 2.37595300 & 0.91593500 \\
\hline $\mathrm{H}$ & -6.31554000 & 0.32876300 & 0.73592800 \\
\hline $\mathrm{C}$ & -5.20463400 & 4.03260600 & 0.59849900 \\
\hline $\mathrm{H}$ & -3.23302500 & 3.30129800 & 0.15876500 \\
\hline $\mathrm{C}$ & -6.54114500 & 3.72133500 & 0.87413800 \\
\hline $\mathrm{H}$ & -7.95559700 & 2.10426400 & 1.13128600 \\
\hline $\mathrm{H}$ & -4.88599200 & 5.07858500 & 0.55563000 \\
\hline $\mathrm{C}$ & -1.07987700 & -3.16946100 & 0.11948300 \\
\hline $\mathrm{C}$ & -1.90623500 & -3.49422900 & 1.21016200 \\
\hline $\mathrm{C}$ & -1.19946800 & -3.91225400 & -1.06550800 \\
\hline $\mathrm{C}$ & -2.81898600 & -4.54681800 & 1.12114600 \\
\hline $\mathrm{H}$ & -1.83636700 & -2.92458100 & 2.13987400 \\
\hline $\mathrm{C}$ & -2.11941400 & -4.96326600 & -1.15451900 \\
\hline $\mathrm{H}$ & -0.56796000 & -3.68095300 & -1.92516600 \\
\hline $\mathrm{C}$ & -2.92767700 & -5.28651900 & -0.06176000 \\
\hline $\mathrm{H}$ & -3.45023700 & -4.78888900 & 1.97989100 \\
\hline $\mathrm{H}$ & -2.19788200 & -5.53407100 & -2.08337800 \\
\hline $\mathrm{H}$ & -3.64196300 & -6.11075800 & -0.13065300 \\
\hline $\mathrm{C}$ & 0.92050300 & -2.16634900 & 1.89856000 \\
\hline $\mathrm{C}$ & 1.30617800 & -3.47608200 & 2.23852600 \\
\hline $\mathrm{C}$ & 1.11755900 & -1.14016600 & 2.83668000 \\
\hline $\mathrm{C}$ & 1.90532500 & -3.74243500 & 3.47190000 \\
\hline $\mathrm{H}$ & 1.12201600 & -4.29995400 & 1.54587000 \\
\hline $\mathrm{C}$ & 1.71264000 & -1.40806300 & 4.07461100 \\
\hline $\mathrm{H}$ & 0.79522600 & -0.12330000 & 2.60169700 \\
\hline $\mathrm{C}$ & 2.11374600 & -2.70814500 & 4.39169700 \\
\hline $\mathrm{H}$ & 2.20231500 & -4.76467800 & 3.72014400 \\
\hline $\mathrm{H}$ & 1.85466000 & -0.59657700 & 4.79255300 \\
\hline $\mathrm{H}$ & 2.57728100 & -2.91972100 & 5.35862900 \\
\hline $\mathrm{C}$ & 1.37053100 & 2.73864500 & -1.42728500 \\
\hline $\mathrm{C}$ & 2.22139700 & 3.85110200 & -1.54610000 \\
\hline $\mathrm{C}$ & 0.37690700 & 2.53476500 & -2.39798900 \\
\hline $\mathrm{C}$ & 2.08750300 & 4.73248800 & -2.62147200 \\
\hline $\mathrm{H}$ & 2.98959000 & 4.03701900 & -0.79224200 \\
\hline $\mathrm{C}$ & 0.24653300 & 3.41578800 & -3.47675500 \\
\hline $\mathrm{H}$ & -0.31025200 & 1.69124800 & -2.30216100 \\
\hline $\mathrm{C}$ & 1.10140100 & 4.51504900 & -3.59063200 \\
\hline $\mathrm{H}$ & 2.75305900 & 5.59600900 & -2.70052100 \\
\hline $\mathrm{H}$ & -0.53406400 & 3.24635800 & -4.22257700 \\
\hline $\mathrm{H}$ & 0.99527300 & 5.20800300 & -4.42927200 \\
\hline $\mathrm{C}$ & 1.97344500 & 2.58824200 & 1.41330200 \\
\hline $\mathrm{C}$ & 3.29800600 & 2.84453800 & 1.80627800 \\
\hline $\mathrm{C}$ & 0.91821100 & 3.16170100 & 2.14781700 \\
\hline $\mathrm{C}$ & 3.56268100 & 3.66281900 & 2.90962900 \\
\hline $\mathrm{H}$ & 4.13020600 & 2.40370300 & 1.25400600 \\
\hline $\mathrm{C}$ & 1.18882600 & 3.98307900 & 3.24553200 \\
\hline $\mathrm{H}$ & -0.11621700 & 2.96568800 & 1.85496400 \\
\hline $\mathrm{C}$ & 2.51010300 & 4.23446900 & 3.63035800 \\
\hline $\mathrm{H}$ & 4.59771100 & 3.85375800 & 3.20526800 \\
\hline $\mathrm{H}$ & 0.36048600 & 4.42474600 & 3.80532900 \\
\hline $\mathrm{H}$ & 2.71884200 & 4.87336200 & 4.49234900 \\
\hline $\mathrm{C}$ & -4.13943000 & -0.83983300 & -0.06632300 \\
\hline $\mathrm{C}$ & -4.86176700 & -1.04435200 & -1.42415700 \\
\hline $\mathrm{H}$ & -4.82171500 & -1.16791900 & 0.73997700 \\
\hline $\mathrm{H}$ & -3.28294000 & -1.52648800 & -0.02490800 \\
\hline $\mathrm{C}$ & -5.53314600 & -2.42239700 & -1.48087900 \\
\hline
\end{tabular}




$\begin{array}{lrrr}\mathrm{H} & -5.65785000 & -0.28283300 & -1.50252100 \\ \mathrm{H} & -6.07399900 & -2.56836100 & -2.43091400 \\ \mathrm{H} & -4.78722600 & -3.23054600 & -1.39250600 \\ \mathrm{H} & -6.25889500 & -2.55135100 & -0.66034100 \\ \mathrm{C} & -3.91705500 & -0.84353600 & -2.61451800 \\ \mathrm{H} & -3.10581200 & -1.59267200 & -2.60376400 \\ \mathrm{H} & -4.45494100 & -0.94673100 & -3.57183000 \\ \mathrm{H} & -3.45244900 & 0.15412800 & -2.59310700 \\ \mathrm{H} & -7.27449700 & 4.51253100 & 1.05063200\end{array}$

\section{8cb - B3LYP/tzvp}

Coordinates:

\begin{tabular}{|c|c|c|c|}
\hline $\mathrm{C}$ & 3.80298200 & -0.25228300 & 0.40006100 \\
\hline $\mathrm{C}$ & 4.92486600 & -0.75787000 & -0.31115900 \\
\hline $\mathrm{C}$ & 4.93397000 & -0.16390600 & -1.60364200 \\
\hline $\mathrm{C}$ & 3.82496700 & 0.72077500 & -1.69134900 \\
\hline $\mathrm{C}$ & 3.11221800 & 0.67475300 & -0.45054000 \\
\hline $\mathrm{H}$ & 3.51441200 & -0.52753000 & 1.40122900 \\
\hline $\mathrm{H}$ & 5.62567600 & -1.49033900 & 0.05703300 \\
\hline $\mathrm{H}$ & 5.64292800 & -0.36588500 & -2.39087600 \\
\hline $\mathrm{H}$ & 3.54834900 & 1.30668000 & -2.55202900 \\
\hline $\mathrm{Fe}$ & 3.14426300 & -1.21305100 & -1.30881400 \\
\hline $\mathrm{C}$ & 1.38199900 & -1.65702300 & -2.30418400 \\
\hline $\mathrm{C}$ & 2.47108400 & -2.22013900 & -3.02106700 \\
\hline $\mathrm{C}$ & 1.37032400 & -2.21725100 & -0.98221200 \\
\hline $\mathrm{H}$ & 0.69073800 & -0.91863700 & -2.67749300 \\
\hline $\mathrm{C}$ & 3.14282900 & -3.12976900 & -2.15807500 \\
\hline $\mathrm{H}$ & 2.75683000 & -1.97343800 & -4.03125700 \\
\hline $\mathrm{C}$ & 2.46979800 & -3.13432300 & -0.90658500 \\
\hline $\mathrm{H}$ & 4.03153400 & -3.69244000 & -2.39698400 \\
\hline $\mathrm{H}$ & 2.76407600 & -3.70041200 & -0.03921600 \\
\hline $\mathrm{P}$ & 1.53546800 & 1.52700100 & -0.04334100 \\
\hline $\mathrm{P}$ & 0.11390700 & -1.76721000 & 0.27977800 \\
\hline $\mathrm{Ni}$ & -0.46674400 & 0.37528300 & 0.19656700 \\
\hline $\mathrm{O}$ & -1.59116100 & 1.86491100 & 0.36545200 \\
\hline $\mathrm{C}$ & -2.31496800 & 0.81977100 & 0.23072800 \\
\hline $\mathrm{C}$ & -3.64458500 & 0.56784200 & 0.17544900 \\
\hline $\mathrm{C}$ & -4.62681400 & 1.64486800 & 0.41882300 \\
\hline $\mathrm{C}$ & -5.98591100 & 1.34379300 & 0.64718400 \\
\hline $\mathrm{C}$ & -4.27376400 & 3.01046000 & 0.45613700 \\
\hline $\mathrm{C}$ & -6.92423800 & 2.33613500 & 0.90551600 \\
\hline $\mathrm{H}$ & -6.31740300 & 0.31469500 & 0.63455600 \\
\hline $\mathrm{C}$ & -5.21489400 & 3.99934600 & 0.71063200 \\
\hline $\mathrm{H}$ & -3.24619200 & 3.29504300 & 0.28346200 \\
\hline $\mathrm{C}$ & -6.55049300 & 3.67583100 & 0.93851600 \\
\hline $\mathrm{H}$ & -7.95712600 & 2.05636800 & 1.08123400 \\
\hline $\mathrm{H}$ & -4.89963700 & 5.03685800 & 0.72696600 \\
\hline $\mathrm{C}$ & -1.09465100 & -3.17388700 & 0.13373300 \\
\hline $\mathrm{C}$ & -1.95246500 & -3.44949000 & 1.20371900 \\
\hline $\mathrm{C}$ & -1.18126500 & -3.95534700 & -1.01942800 \\
\hline $\mathrm{C}$ & -2.86202000 & -4.49660700 & 1.12835000 \\
\hline $\mathrm{H}$ & -1.90963200 & -2.84843700 & 2.10375000 \\
\hline $\mathrm{C}$ & -2.09898500 & -5.00072700 & -1.09614700 \\
\hline $\mathrm{H}$ & -0.52644800 & -3.76278700 & -1.85890500 \\
\hline $\mathrm{C}$ & -2.93773200 & -5.27688500 & -0.02271200 \\
\hline $\mathrm{H}$ & -3.51582000 & -4.69976700 & 1.96764000 \\
\hline $\mathrm{H}$ & -2.15238300 & -5.60023800 & -1.99683900 \\
\hline $\mathrm{H}$ & -3.64798500 & -6.09240000 & -0.08174700 \\
\hline $\mathrm{C}$ & 0.90153100 & -2.16788800 & 1.91555200 \\
\hline $\mathrm{C}$ & 1.27672300 & -3.47117500 & 2.26019800 \\
\hline
\end{tabular}




$\begin{array}{lrrr}\mathrm{C} & 1.09234100 & -1.14486600 & 2.84671800 \\ \mathrm{C} & 1.85927900 & -3.73545100 & 3.49424300 \\ \mathrm{H} & 1.09581200 & -4.28905900 & 1.57479900 \\ \mathrm{C} & 1.67026000 & -1.41005400 & 4.08571800 \\ \mathrm{H} & 0.77862600 & -0.13643600 & 2.60761200 \\ \mathrm{C} & 2.06090800 & -2.70437100 & 4.40897000 \\ \mathrm{H} & 2.14631600 & -4.74917700 & 3.74615900 \\ \mathrm{H} & 1.80662000 & -0.60453800 & 4.79662300 \\ \mathrm{H} & 2.50854400 & -2.91322900 & 5.37292100 \\ \mathrm{C} & 1.37943200 & 2.73543100 & -1.44447200 \\ \mathrm{C} & 2.22513700 & 3.84173500 & -1.56753600 \\ \mathrm{C} & 0.39071900 & 2.52712500 & -2.40795300 \\ \mathrm{C} & 2.09134300 & 4.71376200 & -2.64190400 \\ \mathrm{H} & 2.98586200 & 4.03108400 & -0.82090900 \\ \mathrm{C} & 0.25978800 & 3.39840400 & -3.48596300 \\ \mathrm{H} & -0.29242200 & 1.69338100 & -2.30709800 \\ \mathrm{C} & 1.10971900 & 4.49223300 & -3.60493400 \\ \mathrm{H} & 2.74958900 & 5.57026000 & -2.72367700 \\ \mathrm{H} & -0.51545900 & 3.22772700 & -4.22276500 \\ \mathrm{H} & 1.00330500 & 5.17523900 & -4.43890800 \\ \mathrm{C} & 1.98543500 & 2.60584400 & 1.39777500 \\ \mathrm{C} & 3.30407500 & 2.86100000 & 1.78325800 \\ \mathrm{C} & 0.93811900 & 3.18702500 & 2.12271400 \\ \mathrm{C} & 3.57186400 & 3.68899900 & 2.87014300 \\ \mathrm{H} & 4.12671300 & 2.41396200 & 1.24066200 \\ \mathrm{C} & 1.21089800 & 4.01897000 & 3.20324200 \\ \mathrm{H} & -0.08850400 & 2.99129200 & 1.83769500 \\ \mathrm{C} & 2.52707400 & 4.27041600 & 3.58114700 \\ \mathrm{H} & 4.59868100 & 3.87868400 & 3.15911000 \\ \mathrm{H} & 0.39200000 & 4.46578700 & 3.75377100 \\ \mathrm{H} & 2.73686700 & 4.91472600 & 4.42627600 \\ \mathrm{C} & -4.13338500 & -0.82553300 & -0.14603600 \\ \mathrm{C} & -4.83315400 & -0.97509600 & -1.52024200 \\ \mathrm{H} & -4.82115600 & -1.18374200 & 0.62972800 \\ \mathrm{H} & -3.28200800 & -1.50522100 & -0.12374900 \\ \mathrm{C} & -5.50789300 & -2.34592100 & -1.63531600 \\ \mathrm{H} & -5.61400700 & -0.21146100 & -1.58929600 \\ \mathrm{H} & -6.02512200 & -2.45433900 & -2.59254700 \\ \mathrm{H} & -4.77186600 & -3.15168400 & -1.56045000 \\ \mathrm{H} & -6.24358400 & -2.49485300 & -0.84011600 \\ \mathrm{H} & -3.86119200 & -0.74629800 & -2.68114600 \\ \mathrm{H} & -3.07205300 & -1.50511200 & -2.68123700 \\ \mathrm{H} & -3.37856100 & -0.80231900 & -3.64285200 \\ \mathrm{H} & -7.28196200 & 4.44992100 & 1.13658600\end{array}$

8cb - BP86/tzvp-svp

Coordinates:

$\begin{array}{lrrr}\mathrm{C} & 3.76951600 & -0.33287600 & 0.37987000 \\ \mathrm{C} & 4.88790300 & -0.85458800 & -0.35980200 \\ \mathrm{C} & 4.89363300 & -0.23843200 & -1.65997800 \\ \mathrm{C} & 3.78483800 & 0.67449400 & -1.72952900 \\ \mathrm{C} & 3.07804100 & 0.62491300 & -0.46513100 \\ \mathrm{H} & 3.48201500 & -0.61514100 & 1.40055300 \\ \mathrm{H} & 5.59718600 & -1.61157200 & 0.00027500 \\ \mathrm{H} & 5.60787600 & -0.44335200 & -2.46852900 \\ \mathrm{H} & 3.50499000 & 1.29097400 & -2.59267100 \\ \mathrm{Fe} & 3.10993900 & -1.22338700 & -1.33150800 \\ \mathrm{C} & 1.36130500 & -1.59129800 & -2.31910200 \\ \mathrm{C} & 2.45887300 & -2.16619000 & -3.04869200\end{array}$




\begin{tabular}{|c|c|c|c|}
\hline $\mathrm{C}$ & 1.34463300 & -2.17163700 & -0.98749500 \\
\hline $\mathrm{H}$ & 0.66176300 & -0.83107800 & -2.69045100 \\
\hline $\mathrm{C}$ & 3.12721900 & -3.10265600 & -2.18514200 \\
\hline $\mathrm{H}$ & 2.74907700 & -1.91442400 & -4.07724800 \\
\hline $\mathrm{C}$ & 2.44775200 & -3.11132900 & -0.91714100 \\
\hline $\mathrm{H}$ & 4.02108700 & -3.68884500 & -2.43732500 \\
\hline $\mathrm{H}$ & 2.73766600 & -3.70242600 & -0.04015100 \\
\hline $\mathrm{P}$ & 1.51060800 & 1.48977600 & -0.03768000 \\
\hline $\mathrm{P}$ & 0.10416300 & -1.69965600 & 0.28965700 \\
\hline $\mathrm{Ni}$ & -0.45468900 & 0.39988500 & 0.20319700 \\
\hline $\mathrm{O}$ & -1.59533000 & 1.90248900 & 0.35879100 \\
\hline $\mathrm{C}$ & -2.30884900 & 0.83651600 & 0.23699400 \\
\hline $\mathrm{C}$ & -3.64478500 & 0.55768100 & 0.19303400 \\
\hline $\mathrm{C}$ & -4.64048000 & 1.62986000 & 0.42028000 \\
\hline $\mathrm{C}$ & -6.01716500 & 1.31097500 & 0.61244500 \\
\hline $\mathrm{C}$ & -4.29535200 & 3.01167800 & 0.48256800 \\
\hline $\mathrm{C}$ & -6.97944600 & 2.30407600 & 0.85499300 \\
\hline $\mathrm{H}$ & -6.34214100 & 0.26044600 & 0.58601800 \\
\hline $\mathrm{C}$ & -5.26071400 & 4.00053600 & 0.72079200 \\
\hline $\mathrm{H}$ & -3.24723500 & 3.30922800 & 0.33565200 \\
\hline $\mathrm{C}$ & -6.61390100 & 3.66059400 & 0.91055200 \\
\hline $\mathrm{H}$ & -8.03179400 & 2.00940900 & 1.00347900 \\
\hline $\mathrm{H}$ & -4.94916500 & 5.05790300 & 0.75681200 \\
\hline $\mathrm{C}$ & -1.10181400 & -3.11584000 & 0.16606600 \\
\hline $\mathrm{C}$ & -1.91963800 & -3.42899300 & 1.27654900 \\
\hline $\mathrm{C}$ & -1.22833500 & -3.87893200 & -1.01460000 \\
\hline $\mathrm{C}$ & -2.83355400 & -4.49280200 & 1.20998100 \\
\hline $\mathrm{H}$ & -1.84017600 & -2.83962500 & 2.20392400 \\
\hline $\mathrm{C}$ & -2.14935000 & -4.94095100 & -1.07979800 \\
\hline $\mathrm{H}$ & -0.59788400 & -3.65272200 & -1.88817800 \\
\hline $\mathrm{C}$ & -2.95026100 & -5.25379600 & 0.03191800 \\
\hline $\mathrm{H}$ & -3.46019700 & -4.72799700 & 2.08497800 \\
\hline $\mathrm{H}$ & -2.23435300 & -5.53079600 & -2.00645500 \\
\hline $\mathrm{H}$ & -3.66658400 & -6.08900200 & -0.01875800 \\
\hline $\mathrm{C}$ & 0.91271300 & -2.10057700 & 1.92007500 \\
\hline $\mathrm{C}$ & 1.28561700 & -3.41868300 & 2.27161000 \\
\hline $\mathrm{C}$ & 1.12455700 & -1.05952600 & 2.84993700 \\
\hline $\mathrm{C}$ & 1.89257600 & -3.67862900 & 3.51206000 \\
\hline $\mathrm{H}$ & 1.08251900 & -4.25353100 & 1.58274000 \\
\hline $\mathrm{C}$ & 1.72543200 & -1.32204800 & 4.09460000 \\
\hline $\mathrm{H}$ & 0.80477700 & -0.03568900 & 2.59447900 \\
\hline $\mathrm{C}$ & 2.11765300 & -2.63079600 & 4.42445800 \\
\hline $\mathrm{H}$ & 2.18210900 & -4.70934500 & 3.77239800 \\
\hline $\mathrm{H}$ & 1.87952700 & -0.49869200 & 4.80974400 \\
\hline $\mathrm{H}$ & 2.58749200 & -2.83876600 & 5.39894900 \\
\hline $\mathrm{C}$ & 1.36064000 & 2.70032500 & -1.44245400 \\
\hline $\mathrm{C}$ & 2.23203800 & 3.80411100 & -1.57781000 \\
\hline $\mathrm{C}$ & 0.34849600 & 2.49865200 & -2.40547200 \\
\hline $\mathrm{C}$ & 2.09906200 & 4.68146200 & -2.66716500 \\
\hline $\mathrm{H}$ & 3.01404900 & 3.98601300 & -0.82372400 \\
\hline $\mathrm{C}$ & 0.21961200 & 3.37592900 & -3.49716200 \\
\hline $\mathrm{H}$ & -0.35260300 & 1.65649000 & -2.28645500 \\
\hline $\mathrm{C}$ & 1.09498000 & 4.46749300 & -3.63012000 \\
\hline $\mathrm{H}$ & 2.78085100 & 5.54182900 & -2.76164600 \\
\hline $\mathrm{H}$ & -0.57693500 & 3.21016200 & -4.23971400 \\
\hline $\mathrm{H}$ & 0.99004400 & 5.15945700 & -4.48096400 \\
\hline $\mathrm{C}$ & 2.00255700 & 2.57061300 & 1.39287400 \\
\hline $\mathrm{C}$ & 3.34763400 & 2.80895100 & 1.75407500 \\
\hline $\mathrm{C}$ & 0.96666400 & 3.17551900 & 2.14374800 \\
\hline $\mathrm{C}$ & 3.65126400 & 3.64452300 & 2.84438300 \\
\hline $\mathrm{H}$ & 4.16415200 & 2.33786300 & 1.18504800 \\
\hline $\mathrm{C}$ & 1.27704100 & 4.01403400 & 3.22771400 \\
\hline
\end{tabular}




$\begin{array}{lrrr}\mathrm{H} & -0.08487000 & 2.98738800 & 1.87263400 \\ \mathrm{C} & 2.61834700 & 4.24968500 & 3.58168000 \\ \mathrm{H} & 4.70379100 & 3.82302500 & 3.11750000 \\ \mathrm{H} & 0.46255200 & 4.48251000 & 3.80290000 \\ \mathrm{H} & 2.85855500 & 4.90352700 & 4.43521400 \\ \mathrm{C} & -4.11019100 & -0.84971200 & -0.11326400 \\ \mathrm{C} & -4.77850500 & -1.03493000 & -1.50681400 \\ \mathrm{H} & -4.82329200 & -1.20237500 & 0.66810100 \\ \mathrm{H} & -3.23942800 & -1.53234500 & -0.05114300 \\ \mathrm{C} & -5.42962100 & -2.42392200 & -1.61637300 \\ \mathrm{H} & -5.58634800 & -0.27330700 & -1.59985500 \\ \mathrm{H} & -5.93084500 & -2.56340400 & -2.59806800 \\ \mathrm{H} & -4.66993700 & -3.22870400 & -1.50786700 \\ \mathrm{H} & -6.19300200 & -2.57985300 & -0.82397600 \\ \mathrm{C} & -3.78420000 & -0.79473000 & -2.65375800 \\ \mathrm{H} & -2.95839700 & -1.53993700 & -2.62327100 \\ \mathrm{H} & -4.28056700 & -0.88134600 & -3.64395000 \\ \mathrm{H} & -3.32930500 & 0.21498000 & -2.58720200 \\ \mathrm{H} & -7.36965100 & 4.44016900 & 1.09719200\end{array}$

8cb - BP86/tzvp

Coordinates:

$\begin{array}{lrrr}\mathrm{C} & 3.78074600 & -0.31730100 & 0.39633200 \\ \mathrm{C} & 4.90334500 & -0.83609500 & -0.32979900 \\ \mathrm{C} & 4.91065200 & -0.23463800 & -1.63156400 \\ \mathrm{C} & 3.79941000 & 0.66639100 & -1.71426000 \\ \mathrm{C} & 3.08910600 & 0.62479600 & -0.45757000 \\ \mathrm{H} & 3.48902500 & -0.59345000 & 1.40652500 \\ \mathrm{H} & 5.60497800 & -1.58284300 & 0.03509200 \\ \mathrm{H} & 5.61811800 & -0.44596200 & -2.43030500 \\ \mathrm{H} & 3.51761600 & 1.26215100 & -2.57829700 \\ \mathrm{Fe} & 3.12766700 & -1.23997000 & -1.30817100 \\ \mathrm{C} & 1.38459800 & -1.63923600 & -2.30869700 \\ \mathrm{C} & 2.48502700 & -2.22643500 & -3.01435700 \\ \mathrm{C} & 1.35434600 & -2.19179200 & -0.97093500 \\ \mathrm{H} & 0.69842300 & -0.88934200 & -2.69543200 \\ \mathrm{C} & 3.14298000 & -3.14249300 & -2.12916800 \\ \mathrm{H} & 2.78718100 & -1.99017000 & -4.03206200 \\ \mathrm{C} & 2.45292100 & -3.12671500 & -0.87248100 \\ \mathrm{H} & 4.03609300 & -3.72088700 & -2.35568900 \\ \mathrm{H} & 2.73389500 & -3.68880600 & 0.01365900 \\ \mathrm{P} & 1.51626000 & 1.49202400 & -0.04383300 \\ \mathrm{P} & 0.09885900 & -1.70197600 & 0.28787100 \\ \mathrm{Ni} & -0.45390500 & 0.40288000 & 0.19898700 \\ \mathrm{O} & -1.59915800 & 1.90287000 & 0.37696300 \\ \mathrm{C} & -2.31203000 & 0.84104500 & 0.23019900 \\ \mathrm{C} & -3.64610000 & 0.55930700 & 0.16679600 \\ \mathrm{C} & -4.64578700 & 1.61799900 & 0.42172200 \\ \mathrm{C} & -6.01459600 & 1.28798200 & 0.60258800 \\ \mathrm{C} & -4.30996500 & 2.99324600 & 0.52025200 \\ \mathrm{C} & -6.97770000 & 2.26374600 & 0.87222300 \\ \mathrm{H} & -6.33049200 & 0.24534500 & 0.54466500 \\ \mathrm{C} & -5.27619600 & 3.96508000 & 0.78562000 \\ \mathrm{H} & -3.27208700 & 3.29903800 & 0.38472900 \\ \mathrm{C} & -6.62111100 & 3.61398300 & 0.96413900 \\ \mathrm{H} & -8.01968000 & 1.96221400 & 1.01040100 \\ \mathrm{H} & -4.97335700 & 5.01387200 & 0.84953400 \\ \mathrm{C} & -1.11070700 & -3.11981100 & 0.16522800 \\ \mathrm{C} & -1.96374100 & -3.38782700 & 1.25115800 \\ \mathrm{C} & -1.19713400 & -3.91988200 & -0.98484700\end{array}$




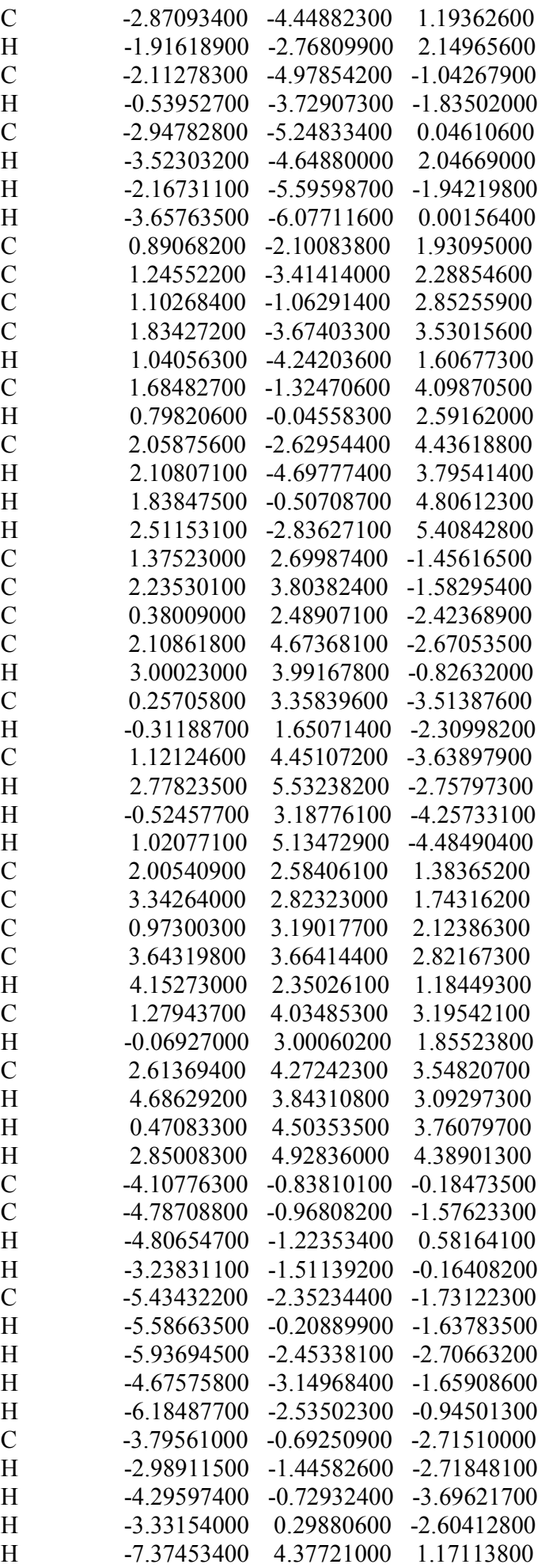

\section{8b CO}

Electronic contribution to gibbs free energy in toluene at 373.15 K (BLYP/6-31g*): -5307.15834746

Electronic contribution to gibbs free energy in toluene at 373.15 K (M06/SDD-6-311+G(2d,p): -4623.97990989 
Energies and corrections (BP86/tzvp-svp):

$\begin{array}{lc}\text { Zero-point correction= } & 0.724994 \text { (Hartree/Particle) } \\ \text { Thermal correction to Energy= } & 0.773304 \\ \text { Thermal correction to Enthalpy= } & 0.774248 \\ \text { Thermal correction to Gibbs Free Energy= } & 0.638588 \\ \text { Sum of electronic and zero-point Energies }= & -5306.762695 \\ \text { Sum of electronic and thermal Energies }= & -5306.714385 \\ \text { Sum of electronic and thermal Enthalpies }= & -5306.713441 \\ \text { Sum of electronic and thermal Free Energies }= & -5306.849101\end{array}$

Coordinates:

\begin{tabular}{|c|c|c|c|}
\hline $\mathrm{C}$ & 3.79605200 & -0.59324700 & 0.21600900 \\
\hline $\mathrm{C}$ & 4.82240400 & -1.22134100 & -0.57293400 \\
\hline $\mathrm{C}$ & 4.79407000 & -0.64276300 & -1.88999300 \\
\hline $\mathrm{C}$ & 3.75678000 & 0.35263000 & -1.92128700 \\
\hline $\mathrm{C}$ & 3.12977000 & 0.39381800 & -0.61530900 \\
\hline $\mathrm{H}$ & 3.55235700 & -0.82400100 & 1.26076900 \\
\hline $\mathrm{H}$ & 5.49287700 & -2.02148500 & -0.23231600 \\
\hline $\mathrm{H}$ & 5.43826100 & -0.92508200 & -2.73329600 \\
\hline $\mathrm{H}$ & 3.47173200 & 0.96601800 & -2.78492700 \\
\hline $\mathrm{Fe}$ & 2.96435600 & -1.47525100 & -1.42393700 \\
\hline $\mathrm{C}$ & 1.14014900 & -1.73191700 & -2.30426100 \\
\hline $\mathrm{C}$ & 2.14720300 & -2.41616000 & -3.06926300 \\
\hline $\mathrm{C}$ & 1.15147800 & -2.26672000 & -0.95380800 \\
\hline $\mathrm{H}$ & 0.48291700 & -0.92987800 & -2.66506700 \\
\hline $\mathrm{C}$ & 2.78632900 & -3.37571300 & -2.20876300 \\
\hline $\mathrm{H}$ & 2.39946100 & -2.22115400 & -4.11992700 \\
\hline $\mathrm{C}$ & 2.17941300 & -3.28935100 & -0.90732100 \\
\hline $\mathrm{H}$ & 3.61606100 & -4.03958600 & -2.48632800 \\
\hline $\mathrm{H}$ & 2.46960500 & -3.87306500 & -0.02545200 \\
\hline $\mathrm{P}$ & 1.66278100 & 1.39210600 & -0.12522100 \\
\hline $\mathrm{P}$ & 0.02213400 & -1.66003000 & 0.36761000 \\
\hline $\mathrm{Ni}$ & -0.37014200 & 0.47377200 & 0.23918600 \\
\hline $\mathrm{O}$ & -1.37787800 & 2.06875600 & 0.38821000 \\
\hline $\mathrm{C}$ & -2.17948300 & 1.06091500 & 0.33574400 \\
\hline $\mathrm{C}$ & -3.53414900 & 0.89206400 & 0.34728100 \\
\hline $\mathrm{C}$ & -4.43494300 & 2.04962300 & 0.54619400 \\
\hline $\mathrm{C}$ & -5.83770200 & 1.85582600 & 0.71559100 \\
\hline $\mathrm{C}$ & -3.97079100 & 3.39673100 & 0.59993200 \\
\hline $\mathrm{C}$ & -6.71295300 & 2.93277800 & 0.92825700 \\
\hline $\mathrm{H}$ & -6.25424200 & 0.83810100 & 0.69117600 \\
\hline $\mathrm{C}$ & -4.84926100 & 4.46945100 & 0.80917800 \\
\hline $\mathrm{H}$ & -2.89800300 & 3.59892300 & 0.47010800 \\
\hline $\mathrm{C}$ & -6.23047700 & 4.25250600 & 0.97653700 \\
\hline $\mathrm{H}$ & -7.78987200 & 2.73360400 & 1.05826500 \\
\hline $\mathrm{H}$ & -4.44607000 & 5.49553200 & 0.84052100 \\
\hline $\mathrm{C}$ & -1.30154600 & -2.97268400 & 0.34598200 \\
\hline $\mathrm{C}$ & -2.08268500 & -3.18842300 & 1.50516100 \\
\hline $\mathrm{C}$ & -1.55093000 & -3.75311400 & -0.80323500 \\
\hline $\mathrm{C}$ & -3.08152600 & -4.17514900 & 1.51687100 \\
\hline $\mathrm{H}$ & -1.90683800 & -2.58383000 & 2.40922300 \\
\hline $\mathrm{C}$ & -2.55638900 & -4.73760600 & -0.78967900 \\
\hline $\mathrm{H}$ & -0.95163000 & -3.60149200 & -1.71406300 \\
\hline $\mathrm{C}$ & -3.32024100 & -4.95489700 & 0.36996700 \\
\hline $\mathrm{H}$ & -3.67842300 & -4.33514800 & 2.42886200 \\
\hline $\mathrm{H}$ & -2.73690700 & -5.34268800 & -1.69252300 \\
\hline $\mathrm{H}$ & -4.10303700 & -5.72974200 & 0.38059700 \\
\hline $\mathrm{C}$ & 0.88058000 & -2.08782700 & 1.96517800 \\
\hline $\mathrm{C}$ & 1.15266000 & -3.42485100 & 2.33738000 \\
\hline $\mathrm{C}$ & 1.23864900 & -1.04616200 & 2.84825000 \\
\hline & 8 & -3.70 & 2 \\
\hline
\end{tabular}




$\begin{array}{lrrr}\mathrm{H} & 0.83734200 & -4.25541600 & 1.68658700 \\ \mathrm{C} & 1.88277500 & -1.32977100 & 4.06641300 \\ \mathrm{H} & 1.00036500 & -0.00425500 & 2.57772300 \\ \mathrm{C} & 2.17247100 & -2.65983400 & 4.41634300 \\ \mathrm{H} & 2.01220100 & -4.75215800 & 3.82773800 \\ \mathrm{H} & 2.15124000 & -0.50535600 & 4.74563600 \\ \mathrm{H} & 2.67561400 & -2.88415300 & 5.37036800 \\ \mathrm{C} & 1.54321100 & 2.59287800 & -1.54130700 \\ \mathrm{C} & 2.49896200 & 3.61454900 & -1.73854900 \\ \mathrm{C} & 0.46842900 & 2.46935400 & -2.44799100 \\ \mathrm{C} & 2.38554700 & 4.48824700 & -2.83294400 \\ \mathrm{H} & 3.33241200 & 3.73562600 & -1.02865000 \\ \mathrm{C} & 0.35905300 & 3.34281700 & -3.54492800 \\ \mathrm{H} & -0.29618500 & 1.69345700 & -2.28078800 \\ \mathrm{C} & 1.31731600 & 4.35240100 & -3.73943500 \\ \mathrm{H} & 3.13346800 & 5.28465900 & -2.97556500 \\ \mathrm{H} & -0.48693700 & 3.23920900 & -4.24272500 \\ \mathrm{H} & 1.22793800 & 5.04180600 & -4.59412900 \\ \mathrm{C} & 2.3111300 & 2.44794100 & 1.26106000 \\ \mathrm{C} & 3.68636800 & 2.57836900 & 1.55767600 \\ \mathrm{C} & 1.36420000 & 3.14706000 & 2.04681000 \\ \mathrm{C} & 4.10741200 & 3.39978900 & 2.61942600 \\ \mathrm{H} & 4.43448900 & 2.03406100 & 0.96064700 \\ \mathrm{C} & 1.79182500 & 3.97093100 & 3.10165000 \\ \mathrm{H} & 0.28948900 & 3.04361100 & 1.82522900 \\ \mathrm{C} & 3.16281600 & 4.09826400 & 3.39182100 \\ \mathrm{H} & 5.18244900 & 3.49356800 & 2.84249600 \\ \mathrm{H} & 1.04599100 & 4.51323800 & 3.70441900 \\ \mathrm{H} & 3.49496300 & 4.74056800 & 4.22300100 \\ \mathrm{C} & -4.11766500 & -0.48372800 & 0.09920000 \\ \mathrm{C} & -4.73537900 & -0.65499300 & -1.30747200 \\ \mathrm{H} & -4.88854400 & -0.72605400 & 0.86786400 \\ \mathrm{H} & -3.31899900 & -1.23859600 & 0.23330500 \\ \mathrm{C} & -5.46423600 & -1.99181800 & -1.51086900 \\ \mathrm{H} & -5.44016500 & 0.18224500 & -1.51207200 \\ \mathrm{H} & -4.76530700 & -2.82832700 & -1.28367500 \\ \mathrm{H} & -6.28403100 & -2.07718700 & -0.76049700 \\ \mathrm{H} & -6.91781600 & 5.09775900 & 1.14032300 \\ \mathrm{H} & -3.92413400 & -0.55131500 & -2.06391300 \\ \mathrm{C} & -6.04166700 & -2.16875000 & -2.92112600 \\ \mathrm{H} & -6.56735500 & -3.14048400 & -3.03405500 \\ \mathrm{H} & -6.77002600 & -1.36529200 & -3.16479500 \\ & -5.24261200 & -2.13046300 & -3.69294500\end{array}$

\section{TS1 (CO to CC)}

Electronic contribution to Gibbs free energy in toluene at 373.15 K (BLYP/6-31g*): -5307.13022000

Energies and corrections (BP86/tzvp-svp):

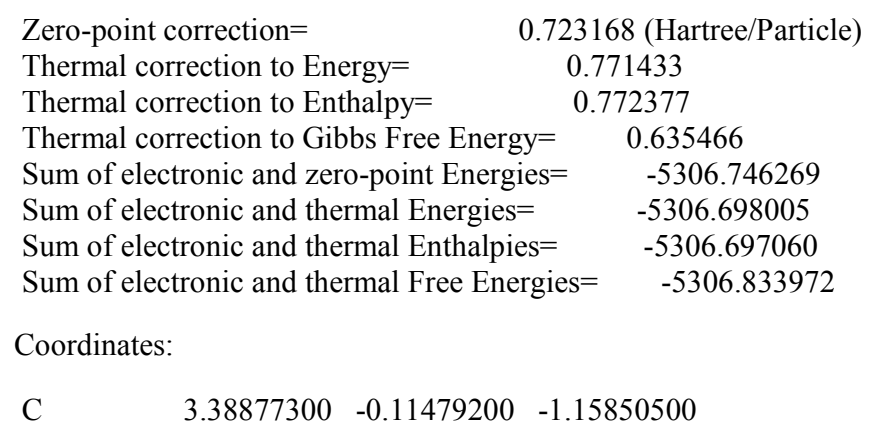




\begin{tabular}{|c|c|c|c|}
\hline $\mathrm{C}$ & 4.72494700 & 0.26704700 & -0.78872500 \\
\hline $\mathrm{C}$ & 5.04791800 & -0.37938800 & 0.45591600 \\
\hline $\mathrm{C}$ & 3.91721500 & -1.17387400 & 0.85670000 \\
\hline $\mathrm{C}$ & 2.88102000 & -1.01944400 & -0.14341300 \\
\hline $\mathrm{H}$ & 2.84420300 & 0.22375200 & -2.04893100 \\
\hline $\mathrm{H}$ & 5.37276800 & 0.95566700 & -1.34721400 \\
\hline $\mathrm{H}$ & 5.98653500 & -0.26979600 & 1.01552300 \\
\hline $\mathrm{H}$ & 3.84049300 & -1.78117700 & 1.76727600 \\
\hline $\mathrm{Fe}$ & 3.36912600 & 0.79743300 & 0.66913400 \\
\hline $\mathrm{C}$ & 2.00448800 & 1.34989500 & 2.08638700 \\
\hline $\mathrm{C}$ & 3.33010600 & 1.69893400 & 2.52235800 \\
\hline $\mathrm{C}$ & 1.74149100 & 2.03155500 & 0.83209200 \\
\hline $\mathrm{H}$ & 1.31058600 & 0.67693700 & 2.60548100 \\
\hline $\mathrm{C}$ & 3.90147800 & 2.58604100 & 1.54429600 \\
\hline $\mathrm{H}$ & 3.82664500 & 1.32962200 & 3.42943700 \\
\hline $\mathrm{C}$ & 2.93129500 & 2.79189900 & 0.50188700 \\
\hline $\mathrm{H}$ & 4.91407900 & 3.01053400 & 1.57160800 \\
\hline $\mathrm{H}$ & 3.06996100 & 3.40159300 & -0.39950500 \\
\hline $\mathrm{P}$ & 1.17944400 & -1.71665400 & -0.13704800 \\
\hline $\mathrm{P}$ & 0.16866200 & 1.88144100 & -0.11801800 \\
\hline $\mathrm{Ni}$ & -0.45018200 & -0.17653800 & -0.33105900 \\
\hline $\mathrm{O}$ & -1.90512900 & -0.08720600 & -2.28541900 \\
\hline $\mathrm{C}$ & -2.16079400 & 0.04161800 & -1.07421100 \\
\hline $\mathrm{C}$ & -3.25964600 & -0.09325800 & -0.23126900 \\
\hline $\mathrm{C}$ & -4.47978200 & -0.73232700 & -0.76146100 \\
\hline $\mathrm{C}$ & -5.69080800 & -0.71509900 & -0.00487600 \\
\hline $\mathrm{C}$ & -4.54073000 & -1.35525600 & -2.04326000 \\
\hline $\mathrm{C}$ & -6.87994300 & -1.26695800 & -0.50543800 \\
\hline $\mathrm{H}$ & -5.70959600 & -0.24087800 & 0.98708100 \\
\hline $\mathrm{C}$ & -5.73066800 & -1.91011500 & -2.53461500 \\
\hline $\mathrm{H}$ & -3.62966200 & -1.39853600 & -2.65804100 \\
\hline $\mathrm{C}$ & -6.91493500 & -1.87347300 & -1.77381100 \\
\hline $\mathrm{H}$ & -7.79411000 & -1.22053900 & 0.10962300 \\
\hline $\mathrm{H}$ & -5.73134700 & -2.38254900 & -3.53106900 \\
\hline $\mathrm{C}$ & -0.90560400 & 3.18138600 & 0.66927300 \\
\hline $\mathrm{C}$ & -2.10831900 & 3.53722100 & 0.01314600 \\
\hline $\mathrm{C}$ & -0.59757800 & 3.78160300 & 1.90948700 \\
\hline $\mathrm{C}$ & -2.97686300 & 4.48078800 & 0.58367600 \\
\hline $\mathrm{H}$ & -2.36864300 & 3.07102700 & -0.95090100 \\
\hline $\mathrm{C}$ & -1.47539500 & 4.72165100 & 2.48161200 \\
\hline $\mathrm{H}$ & 0.33530800 & 3.52071300 & 2.43236000 \\
\hline $\mathrm{C}$ & -2.66459400 & 5.07478500 & 1.82160400 \\
\hline $\mathrm{H}$ & -3.90753300 & 4.75021600 & 0.05931400 \\
\hline $\mathrm{H}$ & -1.22151700 & 5.18390200 & 3.44919600 \\
\hline $\mathrm{H}$ & -3.34964900 & 5.81162000 & 2.27029800 \\
\hline $\mathrm{C}$ & 0.58733900 & 2.68980500 & -1.74199200 \\
\hline $\mathrm{C}$ & 0.80755700 & 4.08309900 & -1.84087700 \\
\hline $\mathrm{C}$ & 0.68527400 & 1.89250500 & -2.90318200 \\
\hline $\mathrm{C}$ & 1.14692000 & 4.66144500 & -3.07581900 \\
\hline $\mathrm{H}$ & 0.70563600 & 4.72182400 & -0.94921600 \\
\hline $\mathrm{C}$ & 1.02257400 & 2.47532500 & -4.13848300 \\
\hline $\mathrm{H}$ & 0.46786800 & 0.81452100 & -2.84006200 \\
\hline $\mathrm{C}$ & 1.25847300 & 3.85808900 & -4.22620400 \\
\hline $\mathrm{H}$ & 1.31863100 & 5.74792600 & -3.14144200 \\
\hline $\mathrm{H}$ & 1.08789200 & 1.84389400 & -5.03880600 \\
\hline $\mathrm{H}$ & 1.51805200 & 4.31466100 & -5.19474400 \\
\hline $\mathrm{C}$ & 1.21251000 & -2.79761100 & 1.37672900 \\
\hline $\mathrm{C}$ & 1.84493400 & -4.06081600 & 1.39392400 \\
\hline $\mathrm{C}$ & 0.59034100 & -2.32544000 & 2.55311400 \\
\hline $\mathrm{C}$ & 1.86504000 & -4.82779200 & 2.57160700 \\
\hline $\mathrm{H}$ & 2.31877000 & -4.45241100 & 0.48018100 \\
\hline $\mathrm{C}$ & 0.61836500 & -3.09024200 & 3.73281000 \\
\hline
\end{tabular}




$\begin{array}{lrrr}\mathrm{H} & 0.07039200 & -1.35367900 & 2.53461200 \\ \mathrm{C} & 1.25641600 & -4.34295700 & 3.74401900 \\ \mathrm{H} & 2.35775600 & -5.81328100 & 2.57222300 \\ \mathrm{H} & 0.13150100 & -2.70857100 & 4.64425500 \\ \mathrm{H} & 1.27260700 & -4.94663900 & 4.66532000 \\ \mathrm{C} & 1.21826000 & -2.93988800 & -1.53218000 \\ \mathrm{C} & 2.41571000 & -3.41193500 & -2.11469000 \\ \mathrm{C} & -0.02140200 & -3.40265500 & -2.03016100 \\ \mathrm{C} & 2.37139500 & -4.34083000 & -3.17001600 \\ \mathrm{H} & 3.38792300 & -3.04773000 & -1.74701400 \\ \mathrm{C} & -0.06127200 & -4.33697700 & -3.07831000 \\ \mathrm{H} & -0.96204900 & -3.02169200 & -1.59935800 \\ \mathrm{C} & 1.13489200 & -4.80718600 & -3.65080600 \\ \mathrm{H} & 3.31069200 & -4.70096500 & -3.61970300 \\ \mathrm{H} & -1.03339700 & -4.69007700 & -3.45714000 \\ \mathrm{H} & 1.10272700 & -5.53322700 & -4.47875200 \\ \mathrm{C} & -3.20992100 & 0.36811800 & 1.21001900 \\ \mathrm{C} & -3.22197300 & -0.75128400 & 2.27512100 \\ \mathrm{H} & -4.06775100 & 1.05446900 & 1.40749800 \\ \mathrm{H} & -2.29863800 & 0.98496200 & 1.35428400 \\ \mathrm{C} & -3.27900200 & -0.22244100 & 3.71678200 \\ \mathrm{H} & -4.08390500 & -1.43259400 & 2.09956000 \\ \mathrm{H} & -2.40177700 & 0.43985000 & 3.90188400 \\ \mathrm{H} & -4.17145900 & 0.43562200 & 3.82745600 \\ \mathrm{H} & -7.84757400 & -2.31289400 & -2.16185100 \\ \mathrm{H} & -2.31531200 & -1.38381100 & 2.13933100 \\ \mathrm{C} & -3.32693300 & -1.32877400 & 4.77842200 \\ \mathrm{H} & -3.35783900 & -0.91289000 & 5.80744400 \\ \mathrm{H} & -4.22416100 & -1.97167700 & 4.65048300 \\ \mathrm{H} & -2.43926200 & -1.99402700 & 4.71147100\end{array}$

\section{$9 \mathrm{CC}$}

Electronic contribution to gibbs free energy in toluene at 373.15 K (BLYP/6-31g*): -5307.15621635

Electronic contribution to gibbs free energy in toluene at 373.15 K (M06/Sdd-6-311+(G(2d,p)): -4623.979936

Energies and corrections (BP86/tzvp-svp):

Zero-point correction $=$

Thermal correction to Energy=

Thermal correction to Enthalpy=

Thermal correction to Gibbs Free Energy=

Sum of electronic and zero-point Energies=

Sum of electronic and thermal Energies=

Sum of electronic and thermal Enthalpies=

Sum of electronic and thermal Free Energies=

\subsection{8 (Hartree/Particle)}

0.772930

0.773874

0.639486

$-5306.766805$

$-5306.718303$

$-5306.717359$

$-5306.851747$

Coordinates:

$\begin{array}{lrrr}\mathrm{C} & -2.53746100 & 1.61751900 & 1.31415600 \\ \mathrm{C} & -3.46549900 & 2.70694600 & 1.16707100 \\ \mathrm{C} & -4.33932400 & 2.40356500 & 0.06547100 \\ \mathrm{C} & -3.96078100 & 1.12343800 & -0.47038500 \\ \mathrm{C} & -2.83826200 & 0.62181800 & 0.30013700 \\ \mathrm{H} & -1.72466900 & 1.56031900 & 2.04934700 \\ \mathrm{H} & -3.48256700 & 3.62124000 & 1.77492100 \\ \mathrm{H} & -5.14199400 & 3.04771700 & -0.31795700 \\ \mathrm{H} & -4.42123500 & 0.62226100 & -1.33044700 \\ \mathrm{Fe} & -2.37000400 & 2.41069900 & -0.55487400 \\ \mathrm{C} & -1.17585900 & 2.05308700 & -2.16580500 \\ \mathrm{C} & -2.12627900 & 3.08291400 & -2.48968700 \\ \mathrm{C} & -0.39000500 & 2.49482600 & -1.02633000\end{array}$




\begin{tabular}{|c|c|c|c|}
\hline $\mathrm{H}$ & -1.08311700 & 1.08420100 & -2.67225700 \\
\hline $\mathrm{C}$ & -1.94624500 & 4.16365800 & -1.55835800 \\
\hline $\mathrm{H}$ & -2.87465100 & 3.03589600 & -3.29173500 \\
\hline $\mathrm{C}$ & -0.88159300 & 3.80958300 & -0.65783400 \\
\hline $\mathrm{H}$ & -2.53572900 & 5.08959500 & -1.52173600 \\
\hline $\mathrm{H}$ & -0.52741300 & 4.41520800 & 0.18481300 \\
\hline $\mathrm{P}$ & -1.84742300 & -0.90883600 & 0.02245400 \\
\hline $\mathrm{P}$ & 0.88398700 & 1.44918500 & -0.19431900 \\
\hline $\mathrm{Ni}$ & 0.34098500 & -0.75256200 & -0.05318800 \\
\hline $\mathrm{O}$ & 0.30672100 & -3.46467200 & -1.18422700 \\
\hline $\mathrm{C}$ & 0.77481800 & -2.51473900 & -0.58272200 \\
\hline $\mathrm{C}$ & 2.00673800 & -1.96431600 & -0.13509300 \\
\hline $\mathrm{C}$ & 2.17961200 & -1.69821700 & 1.30289700 \\
\hline $\mathrm{C}$ & 3.33989100 & -1.03180600 & 1.80367100 \\
\hline $\mathrm{C}$ & 1.21853200 & -2.13765500 & 2.27042200 \\
\hline $\mathrm{C}$ & 3.51779200 & -0.80867300 & 3.17562800 \\
\hline $\mathrm{H}$ & 4.11507900 & -0.69612800 & 1.09908500 \\
\hline $\mathrm{C}$ & 1.39739500 & -1.88860800 & 3.64075600 \\
\hline $\mathrm{H}$ & 0.34760300 & -2.72629900 & 1.94293400 \\
\hline $\mathrm{C}$ & 2.54665300 & -1.22356600 & 4.10828500 \\
\hline $\mathrm{H}$ & 4.43247400 & -0.30196100 & 3.52492900 \\
\hline $\mathrm{H}$ & 0.63757400 & -2.24776300 & 4.35378700 \\
\hline $\mathrm{C}$ & 2.40693700 & 1.80720300 & -1.21253100 \\
\hline $\mathrm{C}$ & 3.68038600 & 1.88650600 & -0.60149600 \\
\hline $\mathrm{C}$ & 2.32448300 & 1.89993300 & -2.62055100 \\
\hline $\mathrm{C}$ & 4.83846300 & 2.05806300 & -1.38014100 \\
\hline $\mathrm{H}$ & 3.76877700 & 1.82168400 & 0.49382800 \\
\hline $\mathrm{C}$ & 3.48415000 & 2.07044100 & -3.39666200 \\
\hline $\mathrm{H}$ & 1.34506600 & 1.84801500 & -3.12045900 \\
\hline $\mathrm{C}$ & 4.74570700 & 2.14935900 & -2.78041800 \\
\hline $\mathrm{H}$ & 5.82109500 & 2.12398200 & -0.88573500 \\
\hline $\mathrm{H}$ & 3.39829700 & 2.14660600 & -4.49255500 \\
\hline $\mathrm{H}$ & 5.65353100 & 2.28534200 & -3.38931200 \\
\hline $\mathrm{C}$ & 1.23266000 & 2.41113400 & 1.36101500 \\
\hline $\mathrm{C}$ & 1.74387800 & 3.73058000 & 1.33788300 \\
\hline $\mathrm{C}$ & 0.98541000 & 1.79496600 & 2.60615200 \\
\hline $\mathrm{C}$ & 1.97173900 & 4.42641700 & 2.53691000 \\
\hline $\mathrm{H}$ & 1.97671000 & 4.21489600 & 0.37636700 \\
\hline $\mathrm{C}$ & 1.21104900 & 2.49482500 & 3.80625600 \\
\hline $\mathrm{H}$ & 0.63272000 & 0.75072100 & 2.63636200 \\
\hline $\mathrm{C}$ & 1.69845000 & 3.81208900 & 3.77398200 \\
\hline $\mathrm{H}$ & 2.36854400 & 5.45396600 & 2.50581700 \\
\hline $\mathrm{H}$ & 1.01552000 & 1.99898400 & 4.77002400 \\
\hline $\mathrm{H}$ & 1.87837400 & 4.35914300 & 4.71328100 \\
\hline $\mathrm{C}$ & -2.62216900 & -1.58021400 & -1.53151500 \\
\hline $\mathrm{C}$ & -3.95586800 & -2.04806700 & -1.54854900 \\
\hline $\mathrm{C}$ & -1.86692500 & -1.61759200 & -2.72183300 \\
\hline $\mathrm{C}$ & -4.52749300 & -2.52058800 & -2.74234700 \\
\hline $\mathrm{H}$ & -4.55071000 & -2.05060400 & -0.62126100 \\
\hline $\mathrm{C}$ & -2.44070000 & -2.08697600 & -3.91685400 \\
\hline $\mathrm{H}$ & -0.81379300 & -1.29377700 & -2.69858300 \\
\hline $\mathrm{C}$ & -3.77235100 & -2.53654300 & -3.92986400 \\
\hline $\mathrm{H}$ & -5.56768500 & -2.88412300 & -2.74371900 \\
\hline $\mathrm{H}$ & -1.83783700 & -2.11496100 & -4.83847600 \\
\hline $\mathrm{H}$ & -4.22052200 & -2.91135400 & -4.86395800 \\
\hline $\mathrm{C}$ & -2.52490000 & -2.07485600 & 1.30937000 \\
\hline $\mathrm{C}$ & -3.22600300 & -1.62396200 & 2.44875500 \\
\hline $\mathrm{C}$ & -2.27334600 & -3.46065100 & 1.16131700 \\
\hline $\mathrm{C}$ & -3.66663500 & -2.53974500 & 3.42250700 \\
\hline $\mathrm{H}$ & -3.43894400 & -0.55190900 & 2.57778600 \\
\hline $\mathrm{C}$ & -2.71887500 & -4.37043200 & 2.13444600 \\
\hline $\mathrm{H}$ & -1.73121800 & -3.82905200 & 0.27582500 \\
\hline
\end{tabular}




$\begin{array}{lrrr}\mathrm{C} & -3.41501800 & -3.91379200 & 3.26954900 \\ \mathrm{H} & -4.21578600 & -2.17258800 & 4.30457900 \\ \mathrm{H} & -2.51993600 & -5.44614600 & 2.00314200 \\ \mathrm{H} & -3.76253400 & -4.62948800 & 4.03156600 \\ \mathrm{C} & 3.16888100 & -1.91028300 & -1.11779100 \\ \mathrm{C} & 4.14507200 & -3.09462000 & -0.95863200 \\ \mathrm{H} & 3.71679900 & -0.94661000 & -1.01990800 \\ \mathrm{H} & 2.76124700 & -1.90935200 & -2.15071100 \\ \mathrm{C} & 5.32227900 & -3.04994000 & -1.94607900 \\ \mathrm{H} & 4.53564300 & -3.11679500 & 0.08369200 \\ \mathrm{H} & 4.92520200 & -3.02599500 & -2.98677800 \\ \mathrm{H} & 5.87255100 & -2.08989100 & -1.81584700 \\ \mathrm{H} & 2.69351100 & -1.04721300 & 5.18542200 \\ \mathrm{H} & 3.58296000 & -4.04630400 & -1.09060700 \\ \mathrm{C} & 6.29529900 & -4.22605500 & -1.79414400 \\ \mathrm{H} & 7.13216800 & -4.16393600 & -2.52109500 \\ \mathrm{H} & 6.73867300 & -4.25546000 & -0.77551300 \\ \mathrm{H} & 5.78242500 & -5.19823200 & -1.95716900\end{array}$

\section{TS2 CC to Carb2}

Electronic Contribution to Gibbs free energy in toluene at 373.15 K (BLYP/6-31g*): -5307.10860642

Electronic Contribution to Gibbs free energy in toluene at $373.15 \mathrm{~K}(\mathrm{M} 06 / 6-311+\mathrm{G}(2 \mathrm{~d}, \mathrm{p}):-4623.92723752$

Energies and corrections (BP86/tzvp-svp):

Zero-point correction $=$

Thermal correction to Energy=

Thermal correction to Enthalpy=

Thermal correction to Gibbs Free Energy=

Sum of electronic and zero-point Energies=

Sum of electronic and thermal Energies $=$

Sum of electronic and thermal Enthalpies=

Sum of electronic and thermal Free Energies=
0.721309 (Hartree/Particle)

0.770027

0.770972

0.634511

$-5306.716559$

$-5306.667841$

$-5306.666897$

$-5306.803357$

Coordinates:

$\begin{array}{lrrr}\mathrm{C} & -2.12842100 & -0.78769900 & -2.24407500 \\ \mathrm{C} & -2.96907700 & -1.76636500 & -2.87928400 \\ \mathrm{C} & -4.04222700 & -2.08750400 & -1.97642700 \\ \mathrm{C} & -3.87395200 & -1.30858700 & -0.77846300 \\ \mathrm{C} & -2.68346300 & -0.49358600 & -0.93326900 \\ \mathrm{H} & -1.21312400 & -0.34801900 & -2.66248600 \\ \mathrm{H} & -2.80394300 & -2.20774300 & -3.87098500 \\ \mathrm{H} & -4.83967700 & -2.82073300 & -2.15742500 \\ \mathrm{H} & -4.51599100 & -1.34860600 & 0.10987500 \\ \mathrm{Fe} & -2.21723600 & -2.46409900 & -1.08764400 \\ \mathrm{C} & -1.40418800 & -3.06457200 & 0.67627300 \\ \mathrm{C} & -2.28116900 & -4.08934800 & 0.17568600 \\ \mathrm{C} & -0.36316500 & -2.82674600 & -0.30912800 \\ \mathrm{H} & -1.53237400 & -2.53105100 & 1.62605700 \\ \mathrm{C} & -1.80756000 & -4.48586300 & -1.12227300 \\ \mathrm{H} & -3.17266100 & -4.47556400 & 0.68728700 \\ \mathrm{C} & -0.63264400 & -3.71414000 & -1.42620300 \\ \mathrm{H} & -2.27368700 & -5.23101800 & -1.78076000 \\ \mathrm{H} & -0.05380500 & -3.76765100 & -2.35563600 \\ \mathrm{P} & -1.87436300 & 0.65210700 & 0.25256000 \\ \mathrm{P} & 0.97204600 & -1.54751700 & -0.16599200 \\ \mathrm{Ni} & 0.34835300 & 0.69510200 & 0.14358100 \\ \mathrm{O} & 0.60242600 & 2.69933500 & 2.21366600 \\ \mathrm{C} & 0.71288100 & 1.91880300 & 1.34282200 \\ \mathrm{C} & 1.92480100 & 1.77714600 & -0.29451100\end{array}$




\begin{tabular}{|c|c|c|c|}
\hline $\mathrm{C}$ & 1.88295000 & 3.17604900 & -0.75264400 \\
\hline $\mathrm{C}$ & 3.07265200 & 3.90952600 & -1.06724900 \\
\hline $\mathrm{C}$ & 0.64947200 & 3.84255500 & -1.03393000 \\
\hline $\mathrm{C}$ & 3.02357100 & 5.19715200 & -1.62460100 \\
\hline $\mathrm{H}$ & 4.05904800 & 3.45677900 & -0.89063500 \\
\hline $\mathrm{C}$ & 0.60368300 & 5.14106700 & -1.54813200 \\
\hline $\mathrm{H}$ & -0.29038500 & 3.30210800 & -0.84374700 \\
\hline $\mathrm{C}$ & 1.79299500 & 5.83470600 & -1.85992400 \\
\hline $\mathrm{H}$ & 3.96727900 & 5.71479400 & -1.86526100 \\
\hline $\mathrm{H}$ & -0.37542200 & 5.61244500 & -1.73292700 \\
\hline $\mathrm{C}$ & 2.08818900 & -2.31623500 & 1.12806000 \\
\hline $\mathrm{C}$ & 3.48374700 & -2.44357400 & 0.93624400 \\
\hline $\mathrm{C}$ & 1.54742100 & -2.69524800 & 2.37950700 \\
\hline $\mathrm{C}$ & 4.30768100 & -2.94682600 & 1.95904300 \\
\hline $\mathrm{H}$ & 3.93879000 & -2.15444200 & -0.02295100 \\
\hline $\mathrm{C}$ & 2.37105800 & -3.20185600 & 3.39845500 \\
\hline $\mathrm{H}$ & 0.46780000 & -2.60148400 & 2.56772200 \\
\hline $\mathrm{C}$ & 3.75644000 & -3.33027400 & 3.19356600 \\
\hline $\mathrm{H}$ & 5.39133800 & -3.04159400 & 1.78327400 \\
\hline $\mathrm{H}$ & 1.92397900 & -3.49711200 & 4.36124900 \\
\hline $\mathrm{H}$ & 4.40281300 & -3.72532200 & 3.99314600 \\
\hline $\mathrm{C}$ & 1.91394600 & -1.84849300 & -1.74610400 \\
\hline $\mathrm{C}$ & 2.46566500 & -3.11167100 & -2.07036100 \\
\hline $\mathrm{C}$ & 2.05847700 & -0.78291400 & -2.65969900 \\
\hline $\mathrm{C}$ & 3.13924800 & -3.30136300 & -3.28842600 \\
\hline $\mathrm{H}$ & 2.37316900 & -3.95309000 & -1.36554000 \\
\hline $\mathrm{C}$ & 2.73113500 & -0.97679500 & -3.88096200 \\
\hline $\mathrm{H}$ & 1.66028700 & 0.20931400 & -2.38337200 \\
\hline $\mathrm{C}$ & 3.26995800 & -2.23468000 & -4.19863500 \\
\hline $\mathrm{H}$ & 3.56620700 & -4.28845500 & -3.52849600 \\
\hline $\mathrm{H}$ & 2.83801200 & -0.13470300 & -4.58336400 \\
\hline $\mathrm{H}$ & 3.79810000 & -2.38651300 & -5.15368900 \\
\hline $\mathrm{C}$ & -2.67324600 & 0.26434100 & 1.89040300 \\
\hline $\mathrm{C}$ & -4.05737400 & 0.44846600 & 2.11450100 \\
\hline $\mathrm{C}$ & -1.86319900 & -0.19389700 & 2.95098800 \\
\hline $\mathrm{C}$ & -4.62026600 & 0.14621200 & 3.36607100 \\
\hline $\mathrm{H}$ & -4.69815100 & 0.84646800 & 1.31172600 \\
\hline $\mathrm{C}$ & -2.42660700 & -0.49135500 & 4.20555100 \\
\hline $\mathrm{H}$ & -0.77786300 & -0.30107100 & 2.78826000 \\
\hline $\mathrm{C}$ & -3.80701600 & -0.32794300 & 4.41268700 \\
\hline $\mathrm{H}$ & -5.70038900 & 0.29106500 & 3.52869600 \\
\hline $\mathrm{H}$ & -1.78083700 & -0.84175400 & 5.02641800 \\
\hline $\mathrm{H}$ & -4.24991900 & -0.55711200 & 5.39502900 \\
\hline $\mathrm{C}$ & -2.68142800 & 2.28010400 & -0.15408200 \\
\hline $\mathrm{C}$ & -3.20270000 & 2.54601400 & -1.44008800 \\
\hline $\mathrm{C}$ & -2.70204400 & 3.30779900 & 0.81925900 \\
\hline $\mathrm{C}$ & -3.73950300 & 3.81021300 & -1.74358300 \\
\hline $\mathrm{H}$ & -3.19633900 & 1.76022600 & -2.21094800 \\
\hline $\mathrm{C}$ & -3.23913500 & 4.56824800 & 0.51161800 \\
\hline $\mathrm{H}$ & -2.29606400 & 3.12391600 & 1.82622600 \\
\hline $\mathrm{C}$ & -3.76056000 & 4.82453100 & -0.77038800 \\
\hline $\mathrm{H}$ & -4.14712000 & 3.99992900 & -2.74955800 \\
\hline $\mathrm{H}$ & -3.24899400 & 5.35685400 & 1.28085300 \\
\hline $\mathrm{H}$ & -4.18288300 & 5.81353900 & -1.00909300 \\
\hline $\mathrm{C}$ & 3.28355100 & 1.22587600 & 0.11062300 \\
\hline $\mathrm{C}$ & 3.95085800 & 1.79429900 & 1.38503400 \\
\hline $\mathrm{H}$ & 3.96585400 & 1.39685500 & -0.75737600 \\
\hline $\mathrm{H}$ & 3.23394500 & 0.12350400 & 0.21566000 \\
\hline $\mathrm{C}$ & 5.41148600 & 1.35106100 & 1.56669200 \\
\hline $\mathrm{H}$ & 3.89675900 & 2.90547400 & 1.37980400 \\
\hline $\mathrm{H}$ & 5.46032900 & 0.23827200 & 1.54923800 \\
\hline $\mathrm{H}$ & 6.00901500 & 1.69074900 & 0.6891730 \\
\hline
\end{tabular}




$\begin{array}{lrrr}\mathrm{H} & 1.75734300 & 6.84880700 & -2.28867900 \\ \mathrm{H} & 3.35561600 & 1.46828300 & 2.26750900 \\ \mathrm{C} & 6.05498600 & 1.87265800 & 2.85803400 \\ \mathrm{H} & 7.10959000 & 1.53945500 & 2.95705400 \\ \mathrm{H} & 6.05080600 & 2.98345900 & 2.89178700 \\ \mathrm{H} & 5.50569300 & 1.51517500 & 3.75544300\end{array}$

\section{0-Cis Carb2}

Electronic Contribution to Gibbs free energy in toluene at 373.15 K (BLYP/6-31g*): -5307.14030316

Electronic Contribution to Gibbs free energy in toluene at 373.15 K (M06/6-311+G(2d,p): -4623.89506701

Energies and corrections (BP86/tzvp-svp):

$\begin{array}{lc}\text { Zero-point correction }= & 0.722116 \text { (Hartree/Particle) } \\ \text { Thermal correction to Energy= } & 0.771386 \\ \text { Thermal correction to Enthalpy= } & 0.772330 \\ \text { Thermal correction to Gibbs Free Energy= } & 0.634130 \\ \text { Sum of electronic and zero-point Energies }= & -5306.739185 \\ \text { Sum of electronic and thermal Energies }= & -5306.689915 \\ \text { Sum of electronic and thermal Enthalpies }= & -5306.688970 \\ \text { Sum of electronic and thermal Free Energies }= & -5306.827170 \\ & \end{array}$

Coordinates: 


\begin{tabular}{|c|c|c|}
\hline-4.02298900 & 0.85104300 & -1.33728600 \\
\hline-4.75930300 & 2.67071900 & 0.67416700 \\
\hline-3.15264900 & 1.79886000 & 1.84735800 \\
\hline-5.08107500 & 1.75418000 & -1.55457600 \\
\hline-3.74921100 & 0.13452200 & -2.12717400 \\
\hline-5.45554100 & 2.66297300 & -0.55003600 \\
\hline-5.04619500 & 3.37865500 & 1.46834600 \\
\hline-5.62072000 & 1.73924200 & -2.51542200 \\
\hline-6.28804100 & 3.36449000 & -0.71845300 \\
\hline-2.72584100 & -1.35725300 & 1.56999500 \\
\hline-4.10926300 & -1.64925300 & 1.51742300 \\
\hline-1.96569100 & -1.88286400 & 2.63542000 \\
\hline-4.70864200 & -2.45719000 & 2.49895200 \\
\hline-4.72751200 & -1.23532800 & 0.70538400 \\
\hline-2.56417900 & -2.69257300 & 3.61830000 \\
\hline-0.89423100 & -1.63875800 & 2.70196200 \\
\hline-3.93737800 & -2.98328200 & 3.55187300 \\
\hline-5.78798700 & -2.67264300 & 2.44322800 \\
\hline-1.95256700 & -3.08849500 & 4.44478500 \\
\hline-4.40955200 & -3.61165000 & 4.32397100 \\
\hline 2.68379900 & -0.25398000 & -1.46074100 \\
\hline 3.99264500 & -0.73116800 & -1.69869000 \\
\hline 2.09972700 & 0.62298400 & -2.39737400 \\
\hline 4.69346200 & -0.34360900 & -2.85434800 \\
\hline 4.47128500 & -1.40867200 & -0.97450600 \\
\hline 2.79434900 & 1.00016900 & -3.56051400 \\
\hline 1.09321800 & 1.02277500 & -2.19574400 \\
\hline 4.09486400 & 0.51823300 & -3.79125700 \\
\hline 5.71597300 & -0.71830300 & -3.02263600 \\
\hline 2.32060600 & 1.68229300 & -4.28485400 \\
\hline 4.64527700 & 0.81998200 & -4.69670100 \\
\hline 3.05236600 & -1.19252300 & 1.23917700 \\
\hline 3.47213000 & -2.50751400 & 1.53651300 \\
\hline 3.67871000 & -0.10741900 & 1.89608200 \\
\hline 4.49624500 & -2.73254200 & 2.47578300 \\
\hline 2.99820800 & -3.36420300 & 1.03344800 \\
\hline 4.70842800 & -0.33550900 & 2.82290800 \\
\hline 3.35454000 & 0.92412200 & 1.68231600 \\
\hline 5.11822700 & -1.64923100 & 3.11920400 \\
\hline 4.80984600 & -3.76453800 & 2.70191200 \\
\hline 5.18875800 & 0.52027000 & 3.32364100 \\
\hline 5.92006600 & -1.82702700 & 3.85353000 \\
\hline-0.62295200 & 3.04436600 & -0.88257900 \\
\hline-1.22809400 & 4.34944600 & -0.30303100 \\
\hline-1.44348400 & 2.36962100 & -1.19813700 \\
\hline-0.06255600 & 3.32621300 & -1.81049800 \\
\hline-2.19556300 & 5.04729300 & -1.27117100 \\
\hline-1.76038300 & 4.10741000 & 0.64345400 \\
\hline-1.66243300 & 5.26857500 & -2.22442800 \\
\hline-3.01639400 & 4.34403700 & -1.53686800 \\
\hline 4.74223700 & 5.32009500 & 1.19846000 \\
\hline-0.40475900 & 5.04452300 & -0.02681200 \\
\hline-2.79141900 & 6.34130600 & -0.70247700 \\
\hline-3.48403400 & 6.82450700 & -1.42316800 \\
\hline-3.36313400 & 6.14604100 & 0.23003800 \\
\hline 1.99837500 & 7.07920700 & -0.4544131 \\
\hline
\end{tabular}

\section{0-Trans Carb2}

Electronic Contribution to Gibbs free energy in toluene at 373.15 K (BLYP/6-31g*): -5307.1369218

Electronic Contribution to Gibbs free energy in toluene at $373.15 \mathrm{~K}(\mathrm{M} 06 / 6-311+\mathrm{G}(2 \mathrm{~d}, \mathrm{p}):-4623.89539893$ 
Energies and corrections (BP86/tzvp-svp):

$\begin{array}{lc}\text { Zero-point correction= } & 0.722270 \text { (Hartree/Particle) } \\ \text { Thermal correction to Energy= } & 0.771464 \\ \text { Thermal correction to Enthalpy= } & 0.772408 \\ \text { Thermal correction to Gibbs Free Energy= } & 0.635821 \\ \text { Sum of electronic and zero-point Energies }= & -5306.740242 \\ \text { Sum of electronic and thermal Energies }= & -5306.691049 \\ \text { Sum of electronic and thermal Enthalpies }= & -5306.690104 \\ \text { Sum of electronic and thermal Free Energies }= & -5306.826691\end{array}$

Coordinates:

\begin{tabular}{|c|c|c|c|}
\hline $\mathrm{C}$ & -0.20228200 & -3.28293900 & -0.49193400 \\
\hline $\mathrm{C}$ & -0.62843700 & -4.16924700 & -1.54221200 \\
\hline $\mathrm{C}$ & 0.03248000 & -3.77244500 & -2.75672200 \\
\hline $\mathrm{C}$ & 0.87639700 & -2.64565900 & -2.46062600 \\
\hline $\mathrm{C}$ & 0.74163000 & -2.33127100 & -1.05117500 \\
\hline $\mathrm{H}$ & -0.54546900 & -3.31366700 & 0.54907000 \\
\hline $\mathrm{H}$ & -1.35354700 & -4.98703800 & -1.43594800 \\
\hline $\mathrm{H}$ & -0.10016600 & -4.23343100 & -3.74475200 \\
\hline $\mathrm{H}$ & 1.50253600 & -2.10148100 & -3.17812800 \\
\hline $\mathrm{Fe}$ & -1.07159100 & -2.20888400 & -1.99000400 \\
\hline $\mathrm{C}$ & -1.51134400 & -0.26187400 & -2.39437800 \\
\hline $\mathrm{C}$ & -1.93852900 & -1.09164500 & -3.48893900 \\
\hline $\mathrm{C}$ & -2.19384700 & -0.70202900 & -1.18933000 \\
\hline $\mathrm{H}$ & -0.77649700 & 0.55031400 & -2.45069200 \\
\hline $\mathrm{C}$ & -2.87637600 & -2.05526400 & -2.97694700 \\
\hline $\mathrm{H}$ & -1.58665400 & -1.01845700 & -4.52656600 \\
\hline $\mathrm{C}$ & -3.03742600 & -1.82051100 & -1.56655200 \\
\hline $\mathrm{H}$ & -3.36537800 & -2.85063500 & -3.55552300 \\
\hline $\mathrm{H}$ & -3.66684800 & -2.40472600 & -0.88401100 \\
\hline $\mathrm{P}$ & 1.54545700 & -0.95065500 & -0.11776300 \\
\hline $\mathrm{P}$ & -1.89093100 & 0.01879200 & 0.48306900 \\
\hline $\mathrm{Ni}$ & 0.29313600 & 0.56953100 & 1.01806300 \\
\hline $\mathrm{O}$ & 0.66634000 & -0.09979700 & 3.84958100 \\
\hline $\mathrm{C}$ & 0.51992100 & 0.10799800 & 2.70691700 \\
\hline $\mathrm{C}$ & 0.68463100 & 2.39113100 & 0.76115800 \\
\hline $\mathrm{C}$ & 2.01558100 & 2.94456900 & 1.07006400 \\
\hline $\mathrm{C}$ & 2.78112700 & 2.52246000 & 2.19459800 \\
\hline $\mathrm{C}$ & 2.60143300 & 3.94181800 & 0.23051000 \\
\hline $\mathrm{C}$ & 4.04696100 & 3.06137500 & 2.46404000 \\
\hline $\mathrm{H}$ & 2.33993300 & 1.78865800 & 2.88272900 \\
\hline $\mathrm{C}$ & 3.89264500 & 4.43489700 & 0.46893700 \\
\hline $\mathrm{H}$ & 2.04671000 & 4.30778800 & -0.64674200 \\
\hline $\mathrm{C}$ & 4.62209100 & 4.00654200 & 1.59289800 \\
\hline $\mathrm{H}$ & 4.59435900 & 2.73604700 & 3.36358800 \\
\hline $\mathrm{H}$ & 4.32800800 & 5.17533900 & -0.22183100 \\
\hline $\mathrm{C}$ & -3.18737500 & 1.36088500 & 0.56207300 \\
\hline $\mathrm{C}$ & -3.34673200 & 2.05029700 & 1.78866100 \\
\hline $\mathrm{C}$ & -3.99627200 & 1.72976100 & -0.53438600 \\
\hline $\mathrm{C}$ & -4.29981900 & 3.07333600 & 1.91678500 \\
\hline $\mathrm{H}$ & -2.72010600 & 1.77762600 & 2.65419100 \\
\hline $\mathrm{C}$ & -4.94618500 & 2.76175100 & -0.40619800 \\
\hline $\mathrm{H}$ & -3.89215700 & 1.20199500 & -1.49515100 \\
\hline $\mathrm{C}$ & -5.10282000 & 3.43457400 & 0.81768000 \\
\hline $\mathrm{H}$ & -4.41501200 & 3.59446700 & 2.88078800 \\
\hline $\mathrm{H}$ & -5.57217200 & 3.03531200 & -1.27096300 \\
\hline $\mathrm{H}$ & -5.84886100 & 4.23899600 & 0.91715300 \\
\hline $\mathrm{C}$ & -2.74604400 & -1.24600400 & 1.57698700 \\
\hline $\mathrm{C}$ & -4.15491700 & -1.37487000 & 1.59678300 \\
\hline $\mathrm{C}$ & -1.98091900 & -2.08920200 & 2.40967700 \\
\hline & & - 233419800 & , \\
\hline
\end{tabular}




$\begin{array}{lrrr}\mathrm{H} & -4.77605600 & -0.71287300 & 0.97319600 \\ \mathrm{C} & -2.60085700 & -3.04967900 & 3.23002200 \\ \mathrm{H} & -0.88564600 & -1.97935500 & 2.42537300 \\ \mathrm{C} & -4.00034200 & -3.17650000 & 3.23333800 \\ \mathrm{H} & -5.87448100 & -2.41931900 & 2.41833600 \\ \mathrm{H} & -1.98382300 & -3.69403500 & 3.87651200 \\ \mathrm{H} & -4.48832200 & -3.92359100 & 3.87958300 \\ \mathrm{C} & 2.64410500 & -0.25820100 & -1.46360300 \\ \mathrm{C} & 3.80298300 & -0.94124100 & -1.89904900 \\ \mathrm{C} & 2.29451700 & 0.95675000 & -2.08572500 \\ \mathrm{C} & 4.58698800 & -0.41842500 & -2.94171800 \\ \mathrm{H} & 4.09778900 & -1.88730300 & -1.41845800 \\ \mathrm{C} & 3.07299000 & 1.47477300 & -3.13652300 \\ \mathrm{H} & 1.41044100 & 1.50338400 & -1.72051900 \\ \mathrm{C} & 4.22199400 & 0.78874600 & -3.56631100 \\ \mathrm{H} & 5.49097500 & -0.95786600 & -3.26757000 \\ \mathrm{H} & 2.78574700 & 2.42561700 & -3.61352100 \\ \mathrm{H} & 4.83894700 & 1.19768000 & -4.38247200 \\ \mathrm{C} & 2.80190000 & -1.86539700 & 0.91409300 \\ \mathrm{C} & 2.88920200 & -3.27343600 & 0.97197400 \\ \mathrm{C} & 3.69866400 & -1.09398400 & 1.69025300 \\ \mathrm{C} & 3.84733400 & -3.89564900 & 1.79497400 \\ \mathrm{H} & 2.20870800 & -3.89399700 & 0.36973800 \\ \mathrm{C} & 4.66040300 & -1.71728700 & 2.50096900 \\ \mathrm{H} & 3.64483900 & 0.00611300 & 1.65652200 \\ \mathrm{C} & 4.73533600 & -3.12179400 & 2.56046500 \\ \mathrm{H} & 3.89953900 & -4.99586200 & 1.83143900 \\ \mathrm{H} & 5.35391500 & -1.10073900 & 3.09503900 \\ \mathrm{H} & 5.48518800 & -3.61031500 & 3.20295100 \\ \mathrm{C} & -0.30315500 & 3.46045000 & 0.33180400 \\ \mathrm{C} & -0.66809300 & 3.45109100 & -1.17308100 \\ \mathrm{H} & 0.05131900 & 4.48363600 & 0.59956900 \\ \mathrm{H} & -1.24855400 & 3.29523300 & 0.89389100 \\ \mathrm{C} & -1.62950600 & 4.58408800 & -1.56506900 \\ \mathrm{H} & 0.25809000 & 3.53082000 & -1.78704000 \\ \mathrm{H} & -2.55698300 & 4.49567700 & -0.95655200 \\ \mathrm{H} & -1.17513600 & 5.56257700 & -1.28868900 \\ \mathrm{H} & 5.62648200 & 4.41198200 & 1.79355800 \\ \mathrm{H} & -1.12034400 & 2.46856900 & -1.42702000 \\ \mathrm{C} & -1.98773600 & 4.58550000 & -3.05653300 \\ \mathrm{H} & -2.68053900 & 5.41528200 & -3.30906000 \\ \mathrm{H} & -1.08371400 & 4.70180400 & -3.69277700 \\ & -2.48150900 & 3.63597600 & -3.35495100\end{array}$

\section{TS3-Cis Carb 2 to CO Alkene}

Electronic Contribution to Gibbs free energy in toluene at 373.15 K (BLYP/6-31g*): -5307.09935305

Energies and corrections (BP86/tzvp-svp):

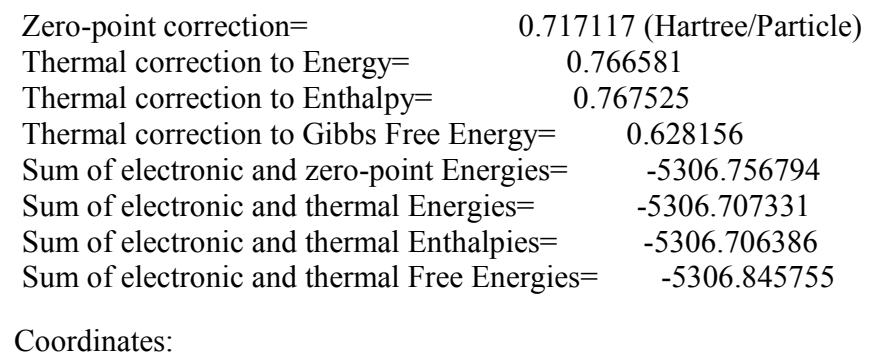




\begin{tabular}{|c|c|c|c|}
\hline $\mathrm{C}$ & -2.05956800 & -2.82205100 & -0.23541700 \\
\hline $\mathrm{C}$ & -2.95799100 & -3.42873500 & -1.18147200 \\
\hline $\mathrm{C}$ & -2.28219800 & -3.50728900 & -2.44944600 \\
\hline $\mathrm{C}$ & -0.96064900 & -2.96049300 & -2.28713300 \\
\hline $\mathrm{C}$ & -0.80969400 & -2.53176700 & -0.91208800 \\
\hline $\mathrm{H}$ & -2.28156500 & -2.60705200 & 0.81750900 \\
\hline $\mathrm{H}$ & -3.98850500 & -3.74770100 & -0.97593800 \\
\hline $\mathrm{H}$ & -2.70549900 & -3.89864200 & -3.38446000 \\
\hline $\mathrm{H}$ & -0.19838400 & -2.87496600 & -3.07152400 \\
\hline $\mathrm{Fe}$ & -2.38515700 & -1.55410000 & -1.80070600 \\
\hline $\mathrm{C}$ & -1.87933000 & 0.31497500 & -2.45508600 \\
\hline $\mathrm{C}$ & -2.81466900 & -0.28622500 & -3.36900700 \\
\hline $\mathrm{C}$ & -2.49314300 & 0.38459200 & -1.14210100 \\
\hline $\mathrm{H}$ & -0.87489300 & 0.67707500 & -2.70986700 \\
\hline $\mathrm{C}$ & -4.01008700 & -0.60141300 & -2.63294300 \\
\hline $\mathrm{H}$ & -2.63791800 & -0.48591200 & -4.43432300 \\
\hline $\mathrm{C}$ & -3.81790100 & -0.18993900 & -1.26696000 \\
\hline $\mathrm{H}$ & -4.90554500 & -1.09377700 & -3.03580100 \\
\hline $\mathrm{H}$ & -4.53799300 & -0.31219500 & -0.44837600 \\
\hline $\mathrm{P}$ & 0.64127100 & -1.76022900 & -0.05852200 \\
\hline $\mathrm{P}$ & -1.63200900 & 0.99788000 & 0.37522500 \\
\hline $\mathrm{Ni}$ & 0.40309400 & 0.23604900 & 0.91195700 \\
\hline $\mathrm{O}$ & 0.66840500 & 0.18120600 & 3.84436700 \\
\hline $\mathrm{C}$ & 0.56482100 & 0.16563800 & 2.67764000 \\
\hline $\mathrm{C}$ & 1.73008900 & 1.52614900 & 0.24045100 \\
\hline $\mathrm{C}$ & 3.00092200 & 1.62444800 & 1.01432300 \\
\hline $\mathrm{C}$ & 3.15613400 & 2.65609800 & 1.97382300 \\
\hline $\mathrm{C}$ & 4.05355600 & 0.69110700 & 0.85201900 \\
\hline $\mathrm{C}$ & 4.32193000 & 2.74194900 & 2.75194900 \\
\hline $\mathrm{H}$ & 2.34270500 & 3.38488800 & 2.11942700 \\
\hline $\mathrm{C}$ & 5.22702800 & 0.79502900 & 1.61775100 \\
\hline $\mathrm{H}$ & 3.94326700 & -0.12448700 & 0.11937200 \\
\hline $\mathrm{C}$ & 5.36692600 & 1.81584600 & 2.57612500 \\
\hline $\mathrm{H}$ & 4.41654300 & 3.54433900 & 3.50172000 \\
\hline $\mathrm{H}$ & 6.03756400 & 0.06294600 & 1.46924000 \\
\hline $\mathrm{C}$ & -1.75245200 & 2.84862000 & 0.14600700 \\
\hline $\mathrm{C}$ & -1.15628200 & 3.66650600 & 1.13563600 \\
\hline $\mathrm{C}$ & -2.36443100 & 3.46824000 & -0.96632500 \\
\hline $\mathrm{C}$ & -1.18645200 & 5.06605900 & 1.02315400 \\
\hline $\mathrm{H}$ & -0.66437700 & 3.19487600 & 2.00194600 \\
\hline $\mathrm{C}$ & -2.38117800 & 4.87153200 & -1.08516300 \\
\hline $\mathrm{H}$ & -2.83774200 & 2.85173500 & -1.74631100 \\
\hline $\mathrm{C}$ & -1.79575200 & 5.67450800 & -0.09142000 \\
\hline $\mathrm{H}$ & -0.72787700 & 5.68736500 & 1.80944000 \\
\hline $\mathrm{H}$ & -2.86319000 & 5.33825600 & -1.95966100 \\
\hline $\mathrm{H}$ & -1.81389500 & 6.77231500 & -0.18292000 \\
\hline $\mathrm{C}$ & -2.98468700 & 0.73383700 & 1.64536500 \\
\hline $\mathrm{C}$ & -4.14720900 & 1.53744300 & 1.68518300 \\
\hline $\mathrm{C}$ & -2.84475300 & -0.30640700 & 2.58813900 \\
\hline $\mathrm{C}$ & -5.14867000 & 1.29703300 & 2.64208900 \\
\hline $\mathrm{H}$ & -4.26964900 & 2.36343600 & 0.96676800 \\
\hline $\mathrm{C}$ & -3.84987500 & -0.55212600 & 3.54116400 \\
\hline $\mathrm{H}$ & -1.92555400 & -0.91414800 & 2.57930500 \\
\hline $\mathrm{C}$ & -5.00432500 & 0.24944100 & 3.57060200 \\
\hline $\mathrm{H}$ & -6.04671900 & 1.93553400 & 2.66483300 \\
\hline $\mathrm{H}$ & -3.72242100 & -1.36744200 & 4.27129900 \\
\hline $\mathrm{H}$ & -5.78846200 & 0.06450500 & 4.32229200 \\
\hline $\mathrm{C}$ & 1.93667200 & -1.81743600 & -1.40625600 \\
\hline $\mathrm{C}$ & 3.12733800 & -2.57093800 & -1.28938600 \\
\hline $\mathrm{C}$ & 1.77840700 & -0.98914600 & -2.54541000 \\
\hline $\mathrm{C}$ & 4.12450100 & -2.49897200 & -2.27963600 \\
\hline & 3.27918400 & -3.22337800 & -0.4163960 \\
\hline
\end{tabular}




$\begin{array}{lrrr}\mathrm{C} & 2.76876800 & -0.93016400 & -3.54190700 \\ \mathrm{H} & 0.85460300 & -0.40310800 & -2.66442800 \\ \mathrm{C} & 3.95073400 & -1.68170200 & -3.41013500 \\ \mathrm{H} & 5.04432300 & -3.09527200 & -2.16617900 \\ \mathrm{H} & 2.61479600 & -0.29151400 & -4.42670000 \\ \mathrm{H} & 4.73122400 & -1.63094200 & -4.18572700 \\ \mathrm{C} & 1.19036300 & -3.18322200 & 1.02101700 \\ \mathrm{C} & 0.84175100 & -4.52565100 & 0.74701800 \\ \mathrm{C} & 2.00173900 & -2.90542800 & 2.14327300 \\ \mathrm{C} & 1.28856400 & -5.56337700 & 1.58394800 \\ \mathrm{H} & 0.21498900 & -4.76355300 & -0.12634900 \\ \mathrm{C} & 2.45830700 & -3.94525300 & 2.97240800 \\ \mathrm{H} & 2.27189200 & -1.86133100 & 2.36786800 \\ \mathrm{C} & 2.09837600 & -5.27674100 & 2.69803600 \\ \mathrm{H} & 1.00203100 & -6.60426700 & 1.36234700 \\ \mathrm{H} & 3.09062800 & -3.70988700 & 3.84342900 \\ \mathrm{H} & 2.44623200 & -6.09118400 & 3.35359700 \\ \mathrm{C} & 1.60298900 & 2.43127300 & -0.84927500 \\ \mathrm{C} & 2.52011900 & 3.55300100 & -1.26084400 \\ \mathrm{H} & 0.65992200 & 2.36605400 & -1.41768100 \\ \mathrm{H} & 1.91526600 & 1.11956400 & -0.97190700 \\ \mathrm{C} & 2.70009700 & 3.68191800 & -2.78536200 \\ \mathrm{H} & 2.06359700 & 4.49302000 & -0.86727200 \\ \mathrm{H} & 3.14950700 & 2.74161900 & -3.17652900 \\ \mathrm{H} & 1.69976300 & 3.76598100 & -3.26709400 \\ \mathrm{H} & 6.28161300 & 1.88633200 & 3.18575200 \\ \mathrm{H} & 3.50077200 & 3.45318100 & -0.75117400 \\ \mathrm{C} & 3.56985900 & 4.87851600 & -3.18918200 \\ \mathrm{H} & 3.68538600 & 4.94261900 & -4.29087300 \\ \mathrm{H} & 3.12617300 & 5.83609000 & -2.84203100 \\ \mathrm{H} & 4.58732800 & 4.80616400 & -2.74950700 \\ & & & \\ & & \end{array}$

\section{TS3-Trans Carb 2 to CO Alkene}

Electronic Contribution to Gibbs free energy in toluene at 373.15 K (BLYP/6-31g*): -5307.09315353

Energies and corrections (BP86/tzvp-svp):

$\begin{array}{lc}\text { Zero-point correction }= & 0.717394 \text { (Hartree/Particle) } \\ \text { Thermal correction to Energy= } & 0.766535 \\ \text { Thermal correction to Enthalpy= } & 0.767480 \\ \text { Thermal correction to Gibbs Free Energy= } & 0.631337 \\ \text { Sum of electronic and zero-point Energies }= & -5306.750505 \\ \text { Sum of electronic and thermal Energies }= & -5306.701363 \\ \text { Sum of electronic and thermal Enthalpies }= & -5306.700419 \\ \text { Sum of electronic and thermal Free Energies }= & -5306.836562\end{array}$

Coordinates:

$\begin{array}{lrrr}\mathrm{C} & 0.23911700 & -3.38262100 & 0.24797900 \\ \mathrm{C} & -0.15504800 & -4.55438200 & -0.48822500 \\ \mathrm{C} & 0.26344400 & -4.38314800 & -1.85422700 \\ \mathrm{C} & 0.92779800 & -3.11077100 & -1.96271300 \\ \mathrm{C} & 0.92049200 & -2.47869700 & -0.65985700 \\ \mathrm{H} & 0.05229300 & -3.20053700 & 1.31358400 \\ \mathrm{H} & -0.70412100 & -5.41419600 & -0.08176100 \\ \mathrm{H} & 0.09122600 & -5.09026200 & -2.67693000 \\ \mathrm{H} & 1.36086900 & -2.68660400 & -2.87711100 \\ \mathrm{Fe} & -0.99981000 & -2.85874700 & -1.28623800 \\ \mathrm{C} & -1.88146800 & -1.24523000 & -2.16917200 \\ \mathrm{C} & -2.33398900 & -2.45532600 & -2.80319700 \\ \mathrm{C} & -2.22477100 & -1.30295800 & -0.75928100\end{array}$




\begin{tabular}{|c|c|c|c|}
\hline $\mathrm{H}$ & -1.36634700 & -0.41651300 & -2.66978400 \\
\hline $\mathrm{C}$ & -2.95119700 & -3.27748800 & -1.79692000 \\
\hline $\mathrm{H}$ & -2.20910700 & -2.71270400 & -3.86347800 \\
\hline $\mathrm{C}$ & -2.88753500 & -2.57477600 & -0.54277100 \\
\hline $\mathrm{H}$ & -3.37468800 & -4.27915400 & -1.95143400 \\
\hline $\mathrm{H}$ & -3.25234500 & -2.94542700 & 0.42303000 \\
\hline $\mathrm{P}$ & 1.63983500 & -0.85193000 & -0.13263000 \\
\hline $\mathrm{P}$ & -1.77282700 & -0.01604800 & 0.49401100 \\
\hline $\mathrm{Ni}$ & 0.31835000 & 0.77595900 & 0.64425300 \\
\hline $\mathrm{O}$ & 0.71358800 & 1.44910400 & 3.49020700 \\
\hline $\mathrm{C}$ & 0.59057700 & 1.15190600 & 2.36482300 \\
\hline $\mathrm{C}$ & 0.68513500 & 2.48050400 & -0.30414700 \\
\hline $\mathrm{C}$ & 1.66723300 & 3.36441300 & 0.39857900 \\
\hline $\mathrm{C}$ & 1.20059500 & 4.55348300 & 1.01114100 \\
\hline $\mathrm{C}$ & 3.04596000 & 3.05699200 & 0.48694800 \\
\hline $\mathrm{C}$ & 2.08219700 & 5.39440500 & 1.71138400 \\
\hline $\mathrm{H}$ & 0.12889600 & 4.80476500 & 0.95303800 \\
\hline $\mathrm{C}$ & 3.92899000 & 3.91509300 & 1.16255400 \\
\hline $\mathrm{H}$ & 3.42453600 & 2.13893700 & 0.01030700 \\
\hline $\mathrm{C}$ & 3.45162700 & 5.08290800 & 1.78727900 \\
\hline $\mathrm{H}$ & 1.69552600 & 6.30500500 & 2.19742400 \\
\hline $\mathrm{H}$ & 5.00151900 & 3.66512300 & 1.20721700 \\
\hline $\mathrm{C}$ & -3.14198400 & 1.23423700 & 0.26430000 \\
\hline $\mathrm{C}$ & -3.07949700 & 2.41827900 & 1.03692100 \\
\hline $\mathrm{C}$ & -4.22677600 & 1.05902300 & -0.62323300 \\
\hline $\mathrm{C}$ & -4.08669800 & 3.39223300 & 0.93820900 \\
\hline $\mathrm{H}$ & -2.23025200 & 2.57254000 & 1.72233100 \\
\hline $\mathrm{C}$ & -5.22858900 & 2.04285600 & -0.73128200 \\
\hline $\mathrm{H}$ & -4.29565500 & 0.14300300 & -1.23075000 \\
\hline $\mathrm{C}$ & -5.16469000 & 3.20900500 & 0.05073700 \\
\hline $\mathrm{H}$ & -4.02782300 & 4.30323200 & 1.55546000 \\
\hline $\mathrm{H}$ & -6.06868300 & 1.89080500 & -1.42830200 \\
\hline $\mathrm{H}$ & -5.95238100 & 3.97500900 & -0.03052200 \\
\hline $\mathrm{C}$ & -2.39666600 & -0.86771300 & 2.04967600 \\
\hline $\mathrm{C}$ & -3.77417300 & -0.97933400 & 2.34736600 \\
\hline $\mathrm{C}$ & -1.46320200 & -1.42276200 & 2.95051500 \\
\hline $\mathrm{C}$ & -4.20374800 & -1.63927400 & 3.51232200 \\
\hline $\mathrm{H}$ & -4.52148900 & -0.54121700 & 1.66732300 \\
\hline $\mathrm{C}$ & -1.89171400 & -2.08909900 & 4.11283400 \\
\hline $\mathrm{H}$ & -0.38748400 & -1.30912700 & 2.74107800 \\
\hline $\mathrm{C}$ & -3.26398800 & -2.19911100 & 4.39713300 \\
\hline $\mathrm{H}$ & -5.28131800 & -1.71197800 & 3.73201100 \\
\hline $\mathrm{H}$ & -1.14643200 & -2.51293400 & 4.80496100 \\
\hline $\mathrm{H}$ & -3.60169600 & -2.71216500 & 5.31191700 \\
\hline $\mathrm{C}$ & 2.60853900 & -0.37980300 & -1.66226500 \\
\hline $\mathrm{C}$ & 4.02032000 & -0.31542800 & -1.69121600 \\
\hline $\mathrm{C}$ & 1.89511000 & 0.03171400 & -2.81529600 \\
\hline $\mathrm{C}$ & 4.69549000 & 0.14229900 & -2.83805600 \\
\hline $\mathrm{H}$ & 4.60145800 & -0.62795200 & -0.81052600 \\
\hline $\mathrm{C}$ & 2.57144700 & 0.47222100 & -3.96655800 \\
\hline $\mathrm{H}$ & 0.79463000 & -0.01097700 & -2.81515200 \\
\hline $\mathrm{C}$ & 3.97692800 & 0.53463800 & -3.98051800 \\
\hline $\mathrm{H}$ & 5.79662100 & 0.18672900 & -2.83683200 \\
\hline $\mathrm{H}$ & 1.99529700 & 0.77202300 & -4.85695400 \\
\hline $\mathrm{H}$ & 4.50845500 & 0.88921900 & -4.87772800 \\
\hline $\mathrm{C}$ & 3.01296500 & -1.46668500 & 0.98081800 \\
\hline $\mathrm{C}$ & 3.58242400 & -2.75363100 & 0.83811700 \\
\hline $\mathrm{C}$ & 3.51232600 & -0.61019900 & 1.98581600 \\
\hline $\mathrm{C}$ & 4.62176800 & -3.17242300 & 1.68691600 \\
\hline $\mathrm{H}$ & 3.21075700 & -3.43575200 & 0.05783800 \\
\hline $\mathrm{C}$ & 4.55881200 & -1.02631000 & 2.82851600 \\
\hline $\mathrm{H}$ & 3.06955200 & 0.39057800 & 2.10941600 \\
\hline
\end{tabular}




$\begin{array}{lrrr}\mathrm{C} & 5.11327300 & -2.31018300 & 2.68435300 \\ \mathrm{H} & 5.05061600 & -4.18062200 & 1.56760800 \\ \mathrm{H} & 4.93440000 & -0.34408600 & 3.60787700 \\ \mathrm{H} & 5.92615900 & -2.64107100 & 3.35054900 \\ \mathrm{C} & 0.23683400 & 3.07204600 & -1.51918900 \\ \mathrm{C} & -0.82085200 & 2.56226900 & -2.45691600 \\ \mathrm{H} & 0.64916800 & 4.06406600 & -1.79176400 \\ \mathrm{H} & 1.14808800 & 2.06093900 & -1.41720600 \\ \mathrm{C} & -1.98384900 & 3.57065100 & -2.59757200 \\ \mathrm{H} & -0.37621600 & 2.40475300 & -3.46741100 \\ \mathrm{H} & -2.50386600 & 3.66305200 & -1.62004600 \\ \mathrm{H} & -1.56850600 & 4.57792400 & -2.82725000 \\ \mathrm{H} & 4.14338300 & 5.74503200 & 2.33176000 \\ \mathrm{H} & -1.20194400 & 1.58734500 & -2.09139700 \\ \mathrm{C} & -2.98308100 & 3.16818100 & -3.68889000 \\ \mathrm{H} & -3.81591000 & 3.89782300 & -3.75790200 \\ \mathrm{H} & -2.49602000 & 3.11731200 & -4.68658500 \\ \mathrm{H} & -3.42905800 & 2.17347000 & -3.47958400\end{array}$

\section{1-Cis CO Alkene}

Electronic Contribution to Gibbs free energy in toluene at 373.15 K (BLYP/6-31g*): -5307.1770937

Energies and corrections (BP86/tzvp-svp):

$\begin{array}{lc}\text { Zero-point correction= } & 0.723149 \text { (Hartree/Particle) } \\ \text { Thermal correction to Energy= } & 0.772066 \\ \text { Thermal correction to Enthalpy= } & 0.773010 \\ \text { Thermal correction to Gibbs Free Energy= } & 0.637627 \\ \text { Sum of electronic and zero-point Energies }= & -5306.825404 \\ \text { Sum of electronic and thermal Energies }= & -5306.776487 \\ \text { Sum of electronic and thermal Enthalpies }= & -5306.775542 \\ \text { Sum of electronic and thermal Free Energies }= & -5306.910925 \\ & \end{array}$

Coordinates:

$\mathrm{C}$

C

C

$\mathrm{H}$

$\mathrm{H}$

$\mathrm{H}$

$\mathrm{H}$

$\mathrm{Fe}$

$\mathrm{C}$

$\mathrm{C}$

C

$\mathrm{H}$

C

$\mathrm{H}$

C

$\mathrm{H}$

$\mathrm{H}$

$\mathrm{P}$

$\mathrm{P}$

$\mathrm{Ni}$

$\mathrm{O}$

C

C

$\mathrm{C}$
$\begin{array}{lll}3.39188500 & -0.40695200 & -0.35728500\end{array}$
$\begin{array}{lll}4.61721700 & -0.20066100 & 0.36616400\end{array}$ $\begin{array}{lll}4.51837700 & -0.90323400 & 1.61678500\end{array}$
$\begin{array}{llll}3.23740500 & -1.55596600 & 1.66460600\end{array}$
$\begin{array}{lll}2.52202200 & -1.25125900 & 0.44159300\end{array}$
$\begin{array}{lll}3.15220700 & 0.01331200 & -1.34148000\end{array}$
$\begin{array}{llll}5.46561200 & 0.41000100 & 0.02991800\end{array}$
$\begin{array}{lll}5.28003000 & -0.92735200 & 2.40775300\end{array}$
$\begin{array}{lll}2.86832800 & -2.17627400 & 2.48978600\end{array}$
$\begin{array}{lll}2.99572700 & 0.47896500 & 1.43603300\end{array}$
$\begin{array}{lll}1.38048900 & 1.21147100 & 2.45671800\end{array}$
$\begin{array}{lll}2.57659000 & 1.41444100 & 3.22981200\end{array}$
$\begin{array}{lll}1.53735700 & 1.88587200 & 1.18109200\end{array}$
$\begin{array}{lll}0.49263100 & 0.64775100 & 2.76926600\end{array}$
$\begin{array}{lll}3.48326800 & 2.20959000 & 2.44538900\end{array}$
$\begin{array}{lll}2.76958400 & 1.01495800 & 4.23431600\end{array}$
$\begin{array}{lll}2.85056700 & 2.50285300 & 1.18635400\end{array}$
$\begin{array}{lll}4.49479300 & 2.51754500 & 2.74266100\end{array}$
$\begin{array}{lll}3.29464900 & 3.06884100 & 0.35807600\end{array}$
$\begin{array}{lll}0.82213700 & -1.73885600 & -0.11166800\end{array}$
$\begin{array}{llll}0.27647600 & 1.82416300 & -0.16199400\end{array}$
$-0.78110900 \quad-0.11669300 \quad-0.50295700$
$\begin{array}{llll}-1.67646300 & 0.07476600 & -3.29610500\end{array}$
$\begin{array}{lll}-1.28974000 & -0.03685400 & -2.19918700\end{array}$
$\begin{array}{lll}-2.51919300 & -1.07758700 & 0.27359600\end{array}$
$\begin{array}{lll}-3.80649700 & -0.92712900 & -0.45230300\end{array}$ 


\begin{tabular}{|c|c|c|c|}
\hline $\mathrm{C}$ & -4.32256300 & 0.30213300 & -0.93595600 \\
\hline $\mathrm{C}$ & -4.58688600 & -2.09222000 & -0.66558100 \\
\hline $\mathrm{C}$ & -5.57324500 & 0.36483200 & -1.56984100 \\
\hline $\mathrm{H}$ & -3.71654000 & 1.21519300 & -0.84278600 \\
\hline $\mathrm{C}$ & -5.83583700 & -2.03225600 & -1.30557100 \\
\hline $\mathrm{H}$ & -4.20198700 & -3.06315400 & -0.31184300 \\
\hline $\mathrm{C}$ & -6.34129300 & -0.80008800 & -1.75579100 \\
\hline $\mathrm{H}$ & -5.94699600 & 1.33419400 & -1.93781100 \\
\hline $\mathrm{H}$ & -6.41767600 & -2.95658700 & -1.45396800 \\
\hline $\mathrm{C}$ & -0.77777400 & 3.31514900 & 0.22217500 \\
\hline $\mathrm{C}$ & -1.81809600 & 3.64115300 & -0.67947800 \\
\hline $\mathrm{C}$ & -0.59979700 & 4.11142300 & 1.37416000 \\
\hline $\mathrm{C}$ & -2.65408900 & 4.74307700 & -0.43761000 \\
\hline $\mathrm{H}$ & -1.96901500 & 3.03010700 & -1.58439800 \\
\hline $\mathrm{C}$ & -1.44693800 & 5.20900800 & 1.62074800 \\
\hline $\mathrm{H}$ & 0.20731800 & 3.87644000 & 2.08526200 \\
\hline $\mathrm{C}$ & -2.47411700 & 5.52865500 & 0.71714000 \\
\hline $\mathrm{H}$ & -3.45451500 & 4.98856400 & -1.15389200 \\
\hline $\mathrm{H}$ & -1.29720600 & 5.81955000 & 2.52589000 \\
\hline $\mathrm{H}$ & -3.13478700 & 6.38879500 & 0.91016900 \\
\hline $\mathrm{C}$ & 1.23766400 & 2.49788000 & -1.61990400 \\
\hline $\mathrm{C}$ & 1.63129100 & 3.85482200 & -1.68205600 \\
\hline $\mathrm{C}$ & 1.57203600 & 1.64470800 & -2.69294200 \\
\hline $\mathrm{C}$ & 2.35967000 & 4.33781700 & -2.78295800 \\
\hline $\mathrm{H}$ & 1.35641400 & 4.54507600 & -0.86887200 \\
\hline $\mathrm{C}$ & 2.30024300 & 2.12797300 & -3.79544400 \\
\hline $\mathrm{H}$ & 1.24342000 & 0.59366100 & -2.66741300 \\
\hline $\mathrm{C}$ & 2.69861100 & 3.47499500 & -3.84153600 \\
\hline $\mathrm{H}$ & 2.65767600 & 5.39817800 & -2.81716900 \\
\hline $\mathrm{H}$ & 2.54775600 & 1.44760700 & -4.62583100 \\
\hline $\mathrm{H}$ & 3.26385600 & 3.85647200 & -4.70687700 \\
\hline $\mathrm{C}$ & 0.33545300 & -2.99493400 & 1.18155600 \\
\hline $\mathrm{C}$ & -0.10971400 & -4.28905700 & 0.82308400 \\
\hline $\mathrm{C}$ & 0.28015700 & -2.62317300 & 2.54818200 \\
\hline $\mathrm{C}$ & -0.57839000 & -5.18573800 & 1.79982700 \\
\hline $\mathrm{H}$ & -0.08263500 & -4.60595500 & -0.23052900 \\
\hline $\mathrm{C}$ & -0.17821100 & -3.52515900 & 3.52397800 \\
\hline $\mathrm{H}$ & 0.61505200 & -1.62179800 & 2.85794300 \\
\hline $\mathrm{C}$ & -0.61150500 & -4.81076600 & 3.15410200 \\
\hline $\mathrm{H}$ & -0.91489400 & -6.19010300 & 1.49598100 \\
\hline $\mathrm{H}$ & -0.19994900 & -3.21686800 & 4.58166000 \\
\hline $\mathrm{H}$ & -0.97541000 & -5.51568000 & 3.91825200 \\
\hline $\mathrm{C}$ & 1.25382200 & -2.85892600 & -1.54404000 \\
\hline $\mathrm{C}$ & 2.49165800 & -3.53862600 & -1.62023500 \\
\hline $\mathrm{C}$ & 0.30477500 & -3.05559700 & -2.57078000 \\
\hline $\mathrm{C}$ & 2.77313800 & -4.38754700 & -2.70425100 \\
\hline $\mathrm{H}$ & 3.24266300 & -3.40272400 & -0.82652600 \\
\hline $\mathrm{C}$ & 0.58316000 & -3.91392100 & -3.65007700 \\
\hline $\mathrm{H}$ & -0.66000900 & -2.52709400 & -2.52298400 \\
\hline $\mathrm{C}$ & 1.81994500 & -4.57813900 & -3.72186100 \\
\hline $\mathrm{H}$ & 3.74458100 & -4.90558000 & -2.75326700 \\
\hline $\mathrm{H}$ & -0.16969100 & -4.05576600 & -4.44182800 \\
\hline $\mathrm{H}$ & 2.04307300 & -5.24335200 & -4.57136300 \\
\hline $\mathrm{C}$ & -1.99092900 & -0.23749800 & 1.27234400 \\
\hline $\mathrm{C}$ & -2.66744000 & 0.95874200 & 1.90854200 \\
\hline $\mathrm{H}$ & -2.18792900 & -2.12497200 & 0.32061300 \\
\hline $\mathrm{H}$ & -1.31327100 & -0.75311000 & 1.96845400 \\
\hline $\mathrm{C}$ & -3.34212900 & 0.60540300 & 3.25783700 \\
\hline $\mathrm{H}$ & -1.92244000 & 1.75794500 & 2.10345600 \\
\hline $\mathrm{H}$ & -2.57291300 & 0.18489400 & 3.94656500 \\
\hline $\mathrm{H}$ & -3.67969400 & 1.55478300 & 3.73099600 \\
\hline $\mathrm{H}$ & -7.31920800 & -0.74844100 & -2.26045600 \\
\hline
\end{tabular}




$\begin{array}{lrrr}\mathrm{H} & -3.41678000 & 1.40908600 & 1.22800600 \\ \mathrm{C} & -4.52511600 & -0.36518900 & 3.15877000 \\ \mathrm{H} & -4.21854800 & -1.34278500 & 2.73179800 \\ \mathrm{H} & -4.97019900 & -0.56059700 & 4.15715800 \\ \mathrm{H} & -5.32649500 & 0.04068400 & 2.50581900\end{array}$

\section{1-Trans CO Alkene}

Electronic Contribution to Gibbs free energy in toluene at 373.15 K (BLYP/6-31g*): -5307.1729805

Energies and corrections (BP86/tzvp-svp):

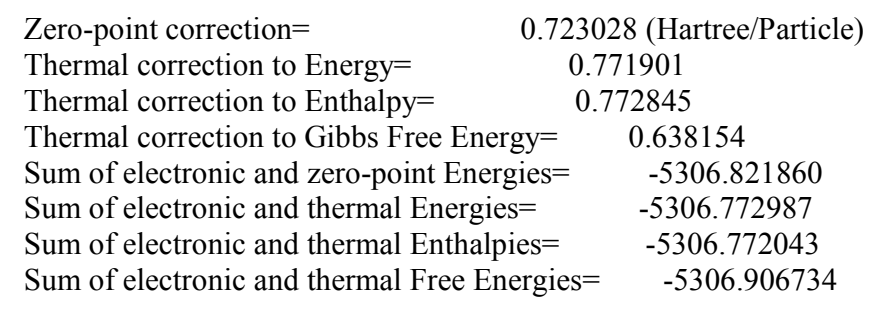

Coordinates:

$\begin{array}{lrrr}\mathrm{C} & -0.15551700 & -3.12574600 & 1.00217400 \\ \mathrm{C} & -0.68200000 & -4.41588600 & 0.64840100 \\ \mathrm{C} & -0.20388500 & -4.74460900 & -0.66692000 \\ \mathrm{C} & 0.62837000 & -3.66409100 & -1.12457700 \\ \mathrm{C} & 0.66255900 & -2.64350100 & -0.09583300 \\ \mathrm{H} & -0.34918000 & -2.59379200 & 1.94085700 \\ \mathrm{H} & -1.35248600 & -5.02678200 & 1.26724200 \\ \mathrm{H} & -0.44233600 & -5.65426900 & -1.23435800 \\ \mathrm{H} & 1.14406900 & -3.62325800 & -2.09106700 \\ \mathrm{Fe} & -1.26621500 & -2.97515600 & -0.70195700 \\ \mathrm{C} & -1.87601600 & -1.52879000 & -2.00928500 \\ \mathrm{C} & -2.41296900 & -2.80195200 & -2.40934700 \\ \mathrm{C} & -2.33851000 & -1.23620500 & -0.66511900 \\ \mathrm{H} & -1.22689400 & -0.88058500 & -2.61022200 \\ \mathrm{C} & -3.20419700 & -3.31238500 & -1.32168500 \\ \mathrm{H} & -2.23176300 & -3.30420700 & -3.36891400 \\ \mathrm{C} & -3.16222400 & -2.35577600 & -0.24738200 \\ \mathrm{H} & -3.72819700 & -4.27747200 & -1.30157000 \\ \mathrm{H} & -3.64510800 & -2.46368400 & 0.73176500 \\ \mathrm{P} & 1.52811200 & -1.00243500 & -0.05795500 \\ \mathrm{P} & -1.83104700 & 0.25490700 & 0.29587300 \\ \mathrm{Ni} & 0.31103400 & 0.92133000 & 0.20985400 \\ \mathrm{O} & 0.59483500 & 2.21745300 & 2.84982200 \\ \mathrm{C} & 0.51918300 & 1.67080700 & 1.82136600 \\ \mathrm{C} & 1.53613700 & 2.24504600 & -0.94208800 \\ \mathrm{C} & 2.16051500 & 3.31726800 & -0.13134500 \\ \mathrm{C} & 1.45824000 & 4.46212800 & 0.32514200 \\ \mathrm{C} & 3.54588000 & 3.23793900 & 0.16219200 \\ \mathrm{C} & 2.10882700 & 5.46771400 & 1.05459700 \\ \mathrm{H} & 0.38493300 & 4.56717500 & 0.10260200 \\ \mathrm{C} & 4.19912900 & 4.24600500 & 0.89011800 \\ \mathrm{H} & 4.11661200 & 2.36615900 & -0.19822800 \\ \mathrm{C} & 3.48362600 & 5.36790800 & 1.34475700 \\ \mathrm{H} & 1.53747300 & 6.34582600 & 1.39726100 \\ \mathrm{H} & 5.27747700 & 4.15591000 & 1.10010500 \\ \mathrm{C} & -3.13281100 & 1.50546400 & -0.17386700 \\ \mathrm{C} & -3.01223200 & 2.81300100 & 0.35112300 \\ \mathrm{C} & -4.22734200 & 1.20510300 & -1.01373100 \\ \mathrm{C} & -3.97170200 & 3.79412900 & 0.05125600 \\ & & & \end{array}$




\begin{tabular}{|c|c|c|c|}
\hline $\mathrm{H}$ & -2.16068700 & 3.06105100 & 1.00546200 \\
\hline $\mathrm{C}$ & -5.18135400 & 2.19314300 & -1.32234900 \\
\hline $\mathrm{H}$ & -4.33747600 & 0.19197700 & -1.43113000 \\
\hline $\mathrm{C}$ & -5.05824800 & 3.48799700 & -0.78976300 \\
\hline $\mathrm{H}$ & -3.86808100 & 4.80629200 & 0.47416500 \\
\hline $\mathrm{H}$ & -6.02901400 & 1.94495300 & -1.98135100 \\
\hline $\mathrm{H}$ & -5.80713500 & 4.25942500 & -1.02986600 \\
\hline $\mathrm{C}$ & -2.45704000 & -0.19844900 & 2.00658700 \\
\hline $\mathrm{C}$ & -3.83904100 & -0.20962800 & 2.30647100 \\
\hline $\mathrm{C}$ & -1.54229500 & -0.53444800 & 3.02697200 \\
\hline $\mathrm{C}$ & -4.29085900 & -0.56537200 & 3.58930500 \\
\hline $\mathrm{H}$ & -4.57227400 & 0.07232500 & 1.53478900 \\
\hline $\mathrm{C}$ & -1.99324000 & -0.89136100 & 4.31071400 \\
\hline $\mathrm{H}$ & -0.46225100 & -0.49869800 & 2.81629200 \\
\hline $\mathrm{C}$ & -3.36944200 & -0.90998900 & 4.59487500 \\
\hline $\mathrm{H}$ & -5.37138400 & -0.56567600 & 3.80563900 \\
\hline $\mathrm{H}$ & -1.26132200 & -1.14573700 & 5.09385200 \\
\hline $\mathrm{H}$ & -3.72433000 & -1.18242100 & 5.60168500 \\
\hline $\mathrm{C}$ & 2.62619600 & -1.11833700 & -1.56903200 \\
\hline $\mathrm{C}$ & 4.02313500 & -0.91740100 & -1.48406900 \\
\hline $\mathrm{C}$ & 2.05465800 & -1.30493000 & -2.85087100 \\
\hline $\mathrm{C}$ & 4.82214900 & -0.91281400 & -2.64231000 \\
\hline $\mathrm{H}$ & 4.49761300 & -0.77009500 & -0.50207600 \\
\hline $\mathrm{C}$ & 2.85512900 & -1.31444400 & -4.00563200 \\
\hline $\mathrm{H}$ & 0.96878000 & -1.45334000 & -2.94839900 \\
\hline $\mathrm{C}$ & 4.24416500 & -1.11533200 & -3.90680700 \\
\hline $\mathrm{H}$ & 5.90878400 & -0.75482800 & -2.55002000 \\
\hline $\mathrm{H}$ & 2.38712500 & -1.47249800 & -4.99062700 \\
\hline $\mathrm{H}$ & 4.87141900 & -1.11553200 & -4.81223800 \\
\hline $\mathrm{C}$ & 2.78018200 & -1.34219900 & 1.29127900 \\
\hline $\mathrm{C}$ & 3.24391500 & -2.64933700 & 1.56779600 \\
\hline $\mathrm{C}$ & 3.30228200 & -0.26275700 & 2.03593000 \\
\hline $\mathrm{C}$ & 4.19969600 & -2.86870200 & 2.57440300 \\
\hline $\mathrm{H}$ & 2.85453500 & -3.50390400 & 0.99291100 \\
\hline $\mathrm{C}$ & 4.26729200 & -0.48205000 & 3.03612400 \\
\hline $\mathrm{H}$ & 2.94711200 & 0.75884600 & 1.82917400 \\
\hline $\mathrm{C}$ & 4.71414500 & -1.78591500 & 3.31160200 \\
\hline $\mathrm{H}$ & 4.54590400 & -3.89381600 & 2.78364400 \\
\hline $\mathrm{H}$ & 4.66367300 & 0.37306800 & 3.60650100 \\
\hline $\mathrm{H}$ & 5.46227600 & -1.96018000 & 4.10151000 \\
\hline $\mathrm{C}$ & 0.24659200 & 2.25852600 & -1.49187600 \\
\hline $\mathrm{C}$ & 0.00152300 & 1.74078400 & -2.90357400 \\
\hline $\mathrm{H}$ & 2.27655300 & 1.61754500 & -1.46136700 \\
\hline $\mathrm{H}$ & -0.42724800 & 3.06754600 & -1.17556700 \\
\hline $\mathrm{C}$ & -1.42836500 & 1.90633500 & -3.44839800 \\
\hline $\mathrm{H}$ & 0.68954400 & 2.28997100 & -3.59176000 \\
\hline $\mathrm{H}$ & -1.49661400 & 1.36313000 & -4.41733800 \\
\hline $\mathrm{H}$ & -2.15164800 & 1.40953600 & -2.76802000 \\
\hline $\mathrm{H}$ & 3.99188400 & 6.16127400 & 1.91542500 \\
\hline $\mathrm{H}$ & 0.31869800 & 0.68052700 & -2.97890400 \\
\hline $\mathrm{C}$ & -1.85792300 & 3.36433500 & -3.66634800 \\
\hline $\mathrm{H}$ & -1.16992100 & 3.88707700 & -4.36583700 \\
\hline $\mathrm{H}$ & -2.88078500 & 3.42358000 & -4.09269600 \\
\hline $\mathrm{H}$ & -1.86821700 & 3.94034000 & -2.71757100 \\
\hline
\end{tabular}

\section{TS4 Alkene Dis}

Electronic Contribution to Gibbs free energy in toluene at 373.15 K (BLYP/6-31g*): -5307.16409782

Energies and corrections (BP86/tzvp-svp): 


$\begin{array}{lc}\text { Zero-point correction= } & 0.721618 \text { (Hartree/Particle) } \\ \text { Thermal correction to Energy= } & 0.770924 \\ \text { Thermal correction to Enthalpy= } & 0.771868 \\ \text { Thermal correction to Gibbs Free Energy= } & 0.630359 \\ \text { Sum of electronic and zero-point Energies }= & -5306.814946 \\ \text { Sum of electronic and thermal Energies }= & -5306.765639 \\ \text { Sum of electronic and thermal Enthalpies }= & -5306.764695 \\ \text { Sum of electronic and thermal Free Energies }= & -5306.906204\end{array}$

Coordinates:

\begin{tabular}{|c|c|c|c|}
\hline & 3.19644900 & 1.69452000 & -0.05809900 \\
\hline 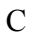 & 4.30884800 & 1.86249700 & -0.95438000 \\
\hline $\mathrm{C}$ & 3.79170300 & 2.27501600 & -2.23179200 \\
\hline $\mathrm{C}$ & 2.35975300 & 2.37502300 & -2.12488000 \\
\hline $\mathrm{C}$ & 1.97713000 & 2.01563100 & -0.77559400 \\
\hline $\mathrm{H}$ & 3.25775100 & 1.37195100 & 0.98922300 \\
\hline $\mathrm{H}$ & 5.36388800 & 1.67973500 & -0.71077300 \\
\hline $\mathrm{H}$ & 4.38256100 & 2.46442400 & -3.13814100 \\
\hline $\mathrm{H}$ & 1.67526500 & 2.66826300 & -2.93038300 \\
\hline $\mathrm{Fe}$ & 2.98600800 & 0.46187300 & -1.67303600 \\
\hline $\mathrm{C}$ & 1.71893200 & -0.97846000 & -2.38824400 \\
\hline $\mathrm{C}$ & 2.82703800 & -0.80946200 & -3.29032900 \\
\hline $\mathrm{C}$ & 2.23691300 & -1.35519400 & -1.08641100 \\
\hline $\mathrm{H}$ & 0.65653400 & -0.85926900 & -2.63832100 \\
\hline $\mathrm{C}$ & 4.03747100 & -1.07176800 & -2.55794600 \\
\hline $\mathrm{H}$ & 2.75988300 & -0.51383000 & -4.34575500 \\
\hline $\mathrm{C}$ & 3.68040100 & -1.40758200 & -1.20414800 \\
\hline $\mathrm{H}$ & 5.05955200 & -1.00189800 & -2.95413900 \\
\hline $\mathrm{H}$ & 4.37973900 & -1.63660800 & -0.39027400 \\
\hline $\mathrm{P}$ & 0.30796500 & 1.93097000 & 0.02511300 \\
\hline $\mathrm{P}$ & 1.16297300 & -1.57971800 & 0.39748100 \\
\hline $\mathrm{Ni}$ & -0.29644500 & -0.00283800 & 0.91097700 \\
\hline $\mathrm{O}$ & -1.80907600 & -0.32542800 & 3.39286100 \\
\hline $\mathrm{C}$ & -1.25555500 & -0.18790400 & 2.36907200 \\
\hline $\mathrm{C}$ & -3.44124300 & -0.07332800 & -0.47565100 \\
\hline $\mathrm{C}$ & -4.62823400 & -0.17699800 & 0.39067900 \\
\hline $\mathrm{C}$ & -4.91630700 & -1.30769500 & 1.19829700 \\
\hline $\mathrm{C}$ & -5.52509000 & 0.92216800 & 0.44884300 \\
\hline $\mathrm{C}$ & -6.07167600 & -1.34943700 & 1.99610600 \\
\hline $\mathrm{H}$ & -4.20685500 & -2.14697500 & 1.23145300 \\
\hline $\mathrm{C}$ & -6.68169600 & 0.87755400 & 1.24139200 \\
\hline $\mathrm{H}$ & -5.30591600 & 1.82046800 & -0.15133600 \\
\hline $\mathrm{C}$ & -6.96486300 & -0.26322800 & 2.01562200 \\
\hline $\mathrm{H}$ & -6.26859400 & -2.23550300 & 2.62107800 \\
\hline $\mathrm{H}$ & -7.36567200 & 1.74152000 & 1.26038500 \\
\hline $\mathrm{C}$ & 0.55693200 & -3.33018800 & 0.18046300 \\
\hline $\mathrm{C}$ & -0.30545500 & -3.85328500 & 1.17318900 \\
\hline $\mathrm{C}$ & 0.89712100 & -4.14121400 & -0.92426600 \\
\hline $\mathrm{C}$ & -0.79934500 & -5.16386000 & 1.07138700 \\
\hline $\mathrm{H}$ & -0.59033700 & -3.22415100 & 2.03224300 \\
\hline $\mathrm{C}$ & 0.38837400 & -5.44980600 & -1.03133000 \\
\hline $\mathrm{H}$ & 1.56682400 & -3.75173600 & -1.70663000 \\
\hline $\mathrm{C}$ & -0.45663200 & -5.96603700 & -0.03416400 \\
\hline $\mathrm{H}$ & -1.46233600 & -5.55963700 & 1.85745600 \\
\hline $\mathrm{H}$ & 0.66107800 & -6.07039500 & -1.90024900 \\
\hline $\mathrm{H}$ & -0.85056100 & -6.99146700 & -0.11775200 \\
\hline $\mathrm{C}$ & 2.42060900 & -1.80324800 & 1.75572300 \\
\hline $\mathrm{C}$ & 3.26431700 & -2.93478500 & 1.82247300 \\
\hline $\mathrm{C}$ & 2.51708200 & -0.81495600 & 2.75931400 \\
\hline $\mathrm{C}$ & 4.19990900 & -3.06222600 & 2.86406200 \\
\hline $\mathrm{H}$ & 3.18322100 & -3.72812800 & 1.06219700 \\
\hline & 3.45481200 & -0.94240900 & 3.79978800 \\
\hline
\end{tabular}




$\begin{array}{lrrr}\mathrm{H} & 1.83266700 & 0.04976400 & 2.72287100 \\ \mathrm{C} & 4.29959600 & -2.06513400 & 3.85229700 \\ \mathrm{H} & 4.85117000 & -3.95009000 & 2.90791600 \\ \mathrm{H} & 3.51742700 & -0.16491700 & 4.57789700 \\ \mathrm{H} & 5.03055600 & -2.16962600 & 4.67005200 \\ \mathrm{C} & -0.81828700 & 2.60897400 & -1.29657300 \\ \mathrm{C} & -1.66768400 & 3.71140300 & -1.04707000 \\ \mathrm{C} & -0.91543500 & 1.95073900 & -2.54634400 \\ \mathrm{C} & -2.57904800 & 4.14870500 & -2.02510300 \\ \mathrm{H} & -1.61666300 & 4.23609500 & -0.08096600 \\ \mathrm{C} & -1.81982200 & 2.39610800 & -3.52526900 \\ \mathrm{H} & -0.26979500 & 1.08546900 & -2.75899500 \\ \mathrm{C} & -2.65730500 & 3.49672000 & -3.26816200 \\ \mathrm{H} & -3.23051800 & 5.01142100 & -1.81158100 \\ \mathrm{H} & -1.87338100 & 1.87487800 & -4.49450600 \\ \mathrm{H} & -3.37031500 & 3.84215700 & -4.03333800 \\ \mathrm{C} & 0.44370000 & 3.37383600 & 1.20148000 \\ \mathrm{C} & 1.20518400 & 4.52318400 & 0.88608000 \\ \mathrm{C} & -0.23787000 & 3.32088000 & 2.43598400 \\ \mathrm{C} & 1.27834800 & 5.59690100 & 1.78922000 \\ \mathrm{H} & 1.74591500 & 4.57882300 & -0.07191900 \\ \mathrm{C} & -0.16773000 & 4.39916900 & 3.33711200 \\ \mathrm{H} & -0.82371400 & 2.42223300 & 2.68571800 \\ \mathrm{C} & 0.59168000 & 5.53774700 & 3.01674400 \\ \mathrm{H} & 1.87665900 & 6.48624800 & 1.53328400 \\ \mathrm{H} & -0.70594000 & 4.34360700 & 4.29687000 \\ \mathrm{H} & 0.65221300 & 6.38004500 & 3.72450500 \\ \mathrm{C} & -2.79301200 & -1.04673000 & -1.15511500 \\ \mathrm{C} & -3.11406400 & -2.51123500 & -1.26909600 \\ \mathrm{H} & -3.07528500 & 0.95184600 & -0.62738500 \\ \mathrm{H} & -1.93752600 & -0.71402400 & -1.76331400 \\ \mathrm{C} & -2.95196000 & -3.04957600 & -2.70795200 \\ \mathrm{H} & -2.43562500 & -3.08922800 & -0.59914300 \\ \mathrm{H} & -1.90869600 & -2.85915200 & -3.04973700 \\ \mathrm{H} & -3.06070300 & -4.15583400 & -2.68585100 \\ \mathrm{H} & -7.86922800 & -0.29852200 & 2.64378800 \\ \mathrm{H} & -4.14648500 & -2.71069900 & -0.90992300 \\ \mathrm{C} & -3.94587500 & -2.45260400 & -3.71257700 \\ \mathrm{H} & -3.84281500 & -1.34881800 & -3.78167900 \\ \mathrm{H} & -3.79685800 & -2.86927200 & -4.73075200 \\ \mathrm{H} & -4.99495900 & -2.66431400 & -3.41330400\end{array}$

\section{Alkene Dis}

Electronic Contribution to Gibbs free energy in toluene at 373.15 K (BLYP/6-31g*): -5307.16718268

Energies and corrections (BP86/tzvp-svp):
Zero-point correction $=$
Thermal correction to Energy=
Thermal correction to Enthalpy=
Thermal correction to Gibbs Free Energy=
Sum of electronic and zero-point Energies=
Sum of electronic and thermal Energies=
Sum of electronic and thermal Enthalpies=
Sum of electronic and thermal Free Energies=

Coordinates:

$\begin{array}{lrrr}\mathrm{C} & 4.51124300 & 0.24783900 & 0.85758100 \\ \mathrm{C} & 5.81364800 & -0.01441700 & 0.30558700\end{array}$




\begin{tabular}{|c|c|c|c|}
\hline $\mathrm{C}$ & 5.94664400 & 0.75137800 & -0.90579900 \\
\hline $\mathrm{C}$ & 4.72980000 & 1.49565600 & -1.10257400 \\
\hline $\mathrm{C}$ & 3.83052200 & 1.19336100 & -0.00779400 \\
\hline $\mathrm{H}$ & 4.09612300 & -0.19756300 & 1.77121100 \\
\hline $\mathrm{H}$ & 6.56262100 & -0.70069600 & 0.72265000 \\
\hline $\mathrm{H}$ & 6.81529700 & 0.75026200 & -1.57807700 \\
\hline $\mathrm{H}$ & 4.50740900 & 2.16029800 & -1.94694000 \\
\hline $\mathrm{Fe}$ & 4.32800300 & -0.51506500 & -1.02946200 \\
\hline $\mathrm{C}$ & 2.85475400 & -1.02576900 & -2.35090300 \\
\hline $\mathrm{C}$ & 4.14590000 & -1.27217600 & -2.93576400 \\
\hline $\mathrm{C}$ & 2.74376900 & -1.81671100 & -1.13907300 \\
\hline $\mathrm{H}$ & 2.08712300 & -0.34813300 & -2.74688200 \\
\hline $\mathrm{C}$ & 4.84814600 & -2.20119000 & -2.09013500 \\
\hline $\mathrm{H}$ & 4.53519700 & -0.80986800 & -3.85262400 \\
\hline $\mathrm{C}$ & 3.98993800 & -2.53886600 & -0.98431800 \\
\hline $\mathrm{H}$ & 5.87056500 & -2.57089600 & -2.24653300 \\
\hline $\mathrm{H}$ & 4.24045700 & -3.20925300 & -0.15251000 \\
\hline $\mathrm{P}$ & 2.10307800 & 1.76345800 & 0.29488100 \\
\hline $\mathrm{P}$ & 1.27079600 & -1.75692800 & -0.02878100 \\
\hline $\mathrm{Ni}$ & 0.57333600 & 0.21726100 & 0.67994200 \\
\hline $\mathrm{O}$ & -1.99121300 & 0.69889300 & 1.99052400 \\
\hline $\mathrm{C}$ & -0.96189600 & 0.50443600 & 1.46263000 \\
\hline $\mathrm{C}$ & -7.06052300 & 0.79906400 & -0.45599800 \\
\hline $\mathrm{C}$ & -7.47516900 & -0.48056000 & 0.14752800 \\
\hline $\mathrm{C}$ & -8.59684600 & -1.23194800 & -0.29089500 \\
\hline $\mathrm{C}$ & -6.69323900 & -1.01722500 & 1.20456300 \\
\hline $\mathrm{C}$ & -8.93892200 & -2.44625800 & 0.32714300 \\
\hline $\mathrm{H}$ & -9.18749800 & -0.88086000 & -1.14934800 \\
\hline $\mathrm{C}$ & -7.03885700 & -2.22723600 & 1.82479800 \\
\hline $\mathrm{H}$ & -5.80406300 & -0.46248600 & 1.54635800 \\
\hline $\mathrm{C}$ & -8.16879900 & -2.94647500 & 1.39251200 \\
\hline $\mathrm{H}$ & -9.81112300 & -3.01381200 & -0.03590500 \\
\hline $\mathrm{H}$ & -6.41918500 & -2.61434000 & 2.64968800 \\
\hline $\mathrm{C}$ & 0.05557600 & -2.82084800 & -0.95927500 \\
\hline $\mathrm{C}$ & -1.29676400 & -2.79128000 & -0.54822800 \\
\hline $\mathrm{C}$ & 0.42204700 & -3.63905000 & -2.05143900 \\
\hline $\mathrm{C}$ & -2.25879900 & -3.57823700 & -1.20270600 \\
\hline $\mathrm{H}$ & -1.59179600 & -2.13716700 & 0.28823800 \\
\hline $\mathrm{C}$ & -0.54545700 & -4.41835800 & -2.71204500 \\
\hline $\mathrm{H}$ & 1.46870100 & -3.66459000 & -2.39301700 \\
\hline $\mathrm{C}$ & -1.88583900 & -4.39234800 & -2.28839200 \\
\hline $\mathrm{H}$ & -3.30845900 & -3.54611300 & -0.86950500 \\
\hline $\mathrm{H}$ & -0.24753100 & -5.04948900 & -3.56495500 \\
\hline $\mathrm{H}$ & -2.64232900 & -5.00175300 & -2.80819900 \\
\hline $\mathrm{C}$ & 1.78356800 & -2.85810900 & 1.38067500 \\
\hline $\mathrm{C}$ & 1.98363100 & -4.24880100 & 1.23567500 \\
\hline $\mathrm{C}$ & 1.95331600 & -2.26747200 & 2.65247200 \\
\hline $\mathrm{C}$ & 2.36604400 & -5.02872100 & 2.34116900 \\
\hline $\mathrm{H}$ & 1.82857900 & -4.72919700 & 0.25637600 \\
\hline $\mathrm{C}$ & 2.33916200 & -3.04788000 & 3.75670500 \\
\hline $\mathrm{H}$ & 1.76144800 & -1.18676500 & 2.77051000 \\
\hline $\mathrm{C}$ & 2.54844000 & -4.42975200 & 3.60162100 \\
\hline $\mathrm{H}$ & 2.51648000 & -6.11344500 & 2.21867100 \\
\hline $\mathrm{H}$ & 2.46664500 & -2.57531900 & 4.74373700 \\
\hline $\mathrm{H}$ & 2.84452400 & -5.04435100 & 4.46676800 \\
\hline $\mathrm{C}$ & 1.72804600 & 2.76744300 & -1.22641400 \\
\hline $\mathrm{C}$ & 2.37294100 & 3.99035000 & -1.51638900 \\
\hline $\mathrm{C}$ & 0.73410800 & 2.28858400 & -2.10878000 \\
\hline $\mathrm{C}$ & 2.04325700 & 4.70753600 & -2.67987500 \\
\hline $\mathrm{H}$ & 3.12928000 & 4.39252300 & -0.82353500 \\
\hline $\mathrm{C}$ & 0.40672900 & 3.00565200 & -3.27320800 \\
\hline $\mathrm{H}$ & 0.20504900 & 1.35139400 & -1.86428300 \\
\hline
\end{tabular}




$\begin{array}{lrrr}\mathrm{C} & 1.06348500 & 4.21506900 & -3.56191000 \\ \mathrm{H} & 2.55077600 & 5.66152500 & -2.89573600 \\ \mathrm{H} & -0.37169700 & 2.62186300 & -3.95183500 \\ \mathrm{H} & 0.80438900 & 4.78137300 & -4.47075900 \\ \mathrm{C} & 2.36438500 & 3.07860900 & 1.59039500 \\ \mathrm{C} & 3.63890900 & 3.58203700 & 1.93431200 \\ \mathrm{C} & 1.22532200 & 3.58112500 & 2.26009300 \\ \mathrm{C} & 3.76845800 & 4.57462800 & 2.92333800 \\ \mathrm{H} & 4.53798400 & 3.19375200 & 1.43070200 \\ \mathrm{C} & 1.35626800 & 4.58036000 & 3.23843100 \\ \mathrm{H} & 0.23088500 & 3.17504400 & 2.01310800 \\ \mathrm{C} & 2.62904200 & 5.07808600 & 3.57490600 \\ \mathrm{H} & 4.76873600 & 4.95574600 & 3.18548700 \\ \mathrm{H} & 0.45895000 & 4.96485200 & 3.74932400 \\ \mathrm{H} & 2.73256800 & 5.85444900 & 4.34977500 \\ \mathrm{C} & -7.81048800 & 1.76012200 & -1.03770200 \\ \mathrm{C} & -9.30365800 & 1.83470700 & -1.21335000 \\ \mathrm{H} & -5.98183200 & 1.00154300 & -0.38129400 \\ \mathrm{H} & -7.26003200 & 2.63265600 & -1.42421600 \\ \mathrm{C} & -9.86309300 & 3.26245500 & -1.02918500 \\ \mathrm{H} & -9.56938400 & 1.48225500 & -2.23868700 \\ \mathrm{H} & -9.35442500 & 3.94612700 & -1.74706400 \\ \mathrm{H} & -10.93612300 & 3.26342700 & -1.32124600 \\ \mathrm{H} & -8.43987500 & -3.89903800 & 1.87499200 \\ \mathrm{H} & -9.81261200 & 1.13909100 & -0.51151800 \\ \mathrm{C} & -9.71666400 & 3.80772600 & 0.39718800 \\ \mathrm{H} & -8.65193200 & 3.85171400 & 0.71016500 \\ \mathrm{H} & -10.13468100 & 4.83211100 & 0.48552100 \\ \mathrm{H} & -10.24760400 & 3.16319600 & 1.13033600\end{array}$

\section{Carb Dis}

Electronic Contribution to Gibbs free energy in toluene at 373.15 K (BLYP/6-31g*): -5307.0680728

Energies and corrections (BP86/tzvp-svp):

$\begin{array}{lc}\text { Zero-point correction= } & 0.718153 \text { (Hartree/Particle) } \\ \text { Thermal correction to Energy= } & 0.769214 \\ \text { Thermal correction to Enthalpy= } & 0.770158 \\ \text { Thermal correction to Gibbs Free Energy= } & 0.619282 \\ \text { Sum of electronic and zero-point Energies }= & -5306.684201 \\ \text { Sum of electronic and thermal Energies }= & -5306.633141 \\ \text { Sum of electronic and thermal Enthalpies }= & -5306.632196 \\ \text { Sum of electronic and thermal Free Energies }= & -5306.783072\end{array}$

Coordinates:

$\begin{array}{lrrr}\mathrm{C} & -3.87043000 & 1.41410000 & 1.00711700 \\ \mathrm{C} & -4.92978700 & 2.23551400 & 0.48459200 \\ \mathrm{C} & -5.56277200 & 1.51630800 & -0.58958600 \\ \mathrm{C} & -4.90221300 & 0.24441700 & -0.72906700 \\ \mathrm{C} & -3.84972200 & 0.16803100 & 0.26339200 \\ \mathrm{H} & -3.18663100 & 1.68482100 & 1.82231700 \\ \mathrm{H} & -5.18987900 & 3.24539300 & 0.82874800 \\ \mathrm{H} & -6.38996300 & 1.88273300 & -1.21248300 \\ \mathrm{H} & -5.13581300 & -0.52784100 & -1.47285700 \\ \mathrm{Fe} & -3.55843800 & 1.77400200 & -0.98042900 \\ \mathrm{C} & -2.20319500 & 1.32979000 & -2.44520200 \\ \mathrm{C} & -3.26282200 & 2.13996500 & -2.98455300 \\ \mathrm{C} & -1.56334800 & 2.06920400 & -1.37306400 \\ \mathrm{H} & -1.92882200 & 0.32106500 & -2.78071300 \\ \mathrm{C} & -3.29846600 & 3.37541300 & -2.24699300\end{array}$




\begin{tabular}{|c|c|c|c|}
\hline $\mathrm{H}$ & -3.94154800 & 1.85239300 & -3.79855100 \\
\hline $\mathrm{C}$ & -2.25520900 & 3.33615200 & -1.25538500 \\
\hline $\mathrm{H}$ & -4.01229900 & 4.19664600 & -2.39699500 \\
\hline $\mathrm{H}$ & -2.03197500 & 4.11912200 & -0.51967300 \\
\hline $\mathrm{P}$ & -2.60650000 & -1.15997700 & 0.56573300 \\
\hline $\mathrm{P}$ & -0.18274600 & 1.38503400 & -0.35680200 \\
\hline $\mathrm{Ni}$ & -0.47510500 & -0.58402300 & 0.60840500 \\
\hline $\mathrm{O}$ & 1.66941600 & -2.18059200 & 1.78221700 \\
\hline $\mathrm{C}$ & 0.80687500 & -1.53845300 & 1.31214200 \\
\hline $\mathrm{C}$ & 6.46907700 & -0.83050000 & -0.49551600 \\
\hline $\mathrm{C}$ & 7.13941400 & -0.13318400 & 0.57346900 \\
\hline $\mathrm{C}$ & 7.81640500 & 1.08036100 & 0.23459100 \\
\hline $\mathrm{C}$ & 7.14952900 & -0.54037700 & 1.94856300 \\
\hline $\mathrm{C}$ & 8.52174700 & 1.81110200 & 1.19857600 \\
\hline $\mathrm{H}$ & 7.77710400 & 1.41113200 & -0.81529600 \\
\hline $\mathrm{C}$ & 7.78349100 & 0.23896600 & 2.92099400 \\
\hline $\mathrm{H}$ & 6.61952400 & -1.45759700 & 2.24927200 \\
\hline $\mathrm{C}$ & 8.48950700 & 1.40273300 & 2.54663200 \\
\hline $\mathrm{H}$ & 9.06587200 & 2.72509100 & 0.91231400 \\
\hline $\mathrm{H}$ & 7.75004800 & -0.06399100 & 3.98000600 \\
\hline $\mathrm{C}$ & 1.26701900 & 1.57662500 & -1.51004000 \\
\hline $\mathrm{C}$ & 2.47413500 & 0.92577300 & -1.16585600 \\
\hline $\mathrm{C}$ & 1.21025600 & 2.32740800 & -2.70588300 \\
\hline $\mathrm{C}$ & 3.60962000 & 1.03355300 & -1.98702100 \\
\hline $\mathrm{H}$ & 2.51643900 & 0.32285800 & -0.24392400 \\
\hline $\mathrm{C}$ & 2.34330900 & 2.42685200 & -3.53455700 \\
\hline $\mathrm{H}$ & 0.27591000 & 2.83299500 & -2.99600400 \\
\hline $\mathrm{C}$ & 3.54153400 & 1.78390700 & -3.17651800 \\
\hline $\mathrm{H}$ & 4.54664200 & 0.51918000 & -1.69872700 \\
\hline $\mathrm{H}$ & 2.28564800 & 3.01143000 & -4.46730800 \\
\hline $\mathrm{H}$ & 4.42651700 & 1.86146000 & -3.82864100 \\
\hline $\mathrm{C}$ & 0.09723400 & 2.73593800 & 0.89171300 \\
\hline $\mathrm{C}$ & 0.61808600 & 4.00264500 & 0.54657900 \\
\hline $\mathrm{C}$ & -0.20714700 & 2.46235800 & 2.24357100 \\
\hline $\mathrm{C}$ & 0.81067200 & 4.98302900 & 1.53571900 \\
\hline $\mathrm{H}$ & 0.88637100 & 4.22143700 & -0.49938400 \\
\hline $\mathrm{C}$ & -0.01747700 & 3.44475000 & 3.23158400 \\
\hline $\mathrm{H}$ & -0.57983800 & 1.45960900 & 2.51583000 \\
\hline $\mathrm{C}$ & 0.48964000 & 4.70784200 & 2.87808000 \\
\hline $\mathrm{H}$ & 1.22072200 & 5.96712600 & 1.25693600 \\
\hline $\mathrm{H}$ & -0.25724600 & 3.21815900 & 4.28280300 \\
\hline $\mathrm{H}$ & 0.64527400 & 5.47705000 & 3.65141000 \\
\hline $\mathrm{C}$ & -2.97615500 & -2.38196900 & -0.78811100 \\
\hline $\mathrm{C}$ & -4.17495300 & -3.12701300 & -0.84351700 \\
\hline $\mathrm{C}$ & -1.99548000 & -2.58203300 & -1.78494200 \\
\hline $\mathrm{C}$ & -4.39496000 & -4.03926700 & -1.89047000 \\
\hline $\mathrm{H}$ & -4.93773300 & -3.00322300 & -0.05821900 \\
\hline $\mathrm{C}$ & -2.21756600 & -3.49299700 & -2.83287400 \\
\hline $\mathrm{H}$ & -1.04480600 & -2.02492300 & -1.72141200 \\
\hline $\mathrm{C}$ & -3.41946600 & -4.22083400 & -2.88850400 \\
\hline $\mathrm{H}$ & -5.33262700 & -4.61717900 & -1.92355200 \\
\hline $\mathrm{H}$ & -1.44385300 & -3.64077800 & -3.60314400 \\
\hline $\mathrm{H}$ & -3.59321900 & -4.93917000 & -3.70562200 \\
\hline $\mathrm{C}$ & -3.31615000 & -2.00060600 & 2.07142100 \\
\hline $\mathrm{C}$ & -4.61648000 & -1.74292100 & 2.56019500 \\
\hline $\mathrm{C}$ & -2.49977100 & -2.93160000 & 2.75350700 \\
\hline $\mathrm{C}$ & -5.09183600 & -2.41041900 & 3.70418600 \\
\hline $\mathrm{H}$ & -5.26174000 & -1.01235600 & 2.04774600 \\
\hline $\mathrm{C}$ & -2.98100000 & -3.60552000 & 3.88798000 \\
\hline $\mathrm{H}$ & -1.47588200 & -3.11847600 & 2.39079200 \\
\hline $\mathrm{C}$ & -4.27793300 & -3.34459800 & 4.36841300 \\
\hline $\mathrm{H}$ & -6.10644600 & -2.19698800 & 4.07777300 \\
\hline
\end{tabular}




$\begin{array}{lrrr}\mathrm{H} & -2.33443100 & -4.33137500 & 4.40679600 \\ \mathrm{H} & -4.65146500 & -3.86557800 & 5.26449500 \\ \mathrm{C} & 6.21478800 & -2.27121500 & -0.32092900 \\ \mathrm{C} & 6.00542900 & -3.09146500 & -1.60999600 \\ \mathrm{H} & 5.21605900 & -2.20535700 & 0.20305200 \\ \mathrm{H} & 6.88152900 & -2.81843700 & 0.39608500 \\ \mathrm{C} & 5.52067000 & -4.52650400 & -1.35529500 \\ \mathrm{H} & 5.28817400 & -2.54949400 & -2.26416900 \\ \mathrm{H} & 6.23710000 & -5.04290500 & -0.67579500 \\ \mathrm{H} & 4.55497800 & -4.49272600 & -0.80057200 \\ \mathrm{H} & 9.00476000 & 2.00129300 & 3.31504500 \\ \mathrm{H} & 6.96764200 & -3.11784800 & -2.17026100 \\ \mathrm{C} & 5.34938800 & -5.34772400 & -2.63929100 \\ \mathrm{H} & 4.99653500 & -6.37758700 & -2.42279300 \\ \mathrm{H} & 4.61085800 & -4.87815000 & -3.32386800 \\ \mathrm{H} & 6.30727600 & -5.43272800 & -3.19643000\end{array}$

\section{CO PD}

Electronic Contribution to Gibbs free energy in toluene at 373.15 K (BLYP/6-31g*): -5307.11495843

Energies and corrections (BP86/tzvp-svp):

$\begin{array}{lc}\text { Zero-point correction= } & 0.722261 \text { (Hartree/Particle) } \\ \text { Thermal correction to Energy= } & 0.771387 \\ \text { Thermal correction to Enthalpy= } & 0.772331 \\ \text { Thermal correction to Gibbs Free Energy= } & 0.630514 \\ \text { Sum of electronic and zero-point Energies }= & -5306.725650 \\ \text { Sum of electronic and thermal Energies }= & -5306.676523 \\ \text { Sum of electronic and thermal Enthalpies }= & -5306.675579 \\ \text { Sum of electronic and thermal Free Energies }= & -5306.817397\end{array}$

Coordinates:

$\begin{array}{lrrr}\mathrm{C} & -0.20229700 & -1.93372200 & -1.96551800 \\ \mathrm{C} & 1.15765900 & -2.31481600 & -2.22828600 \\ \mathrm{C} & 1.58881100 & -3.20126000 & -1.17853600 \\ \mathrm{C} & 0.49282800 & -3.38415800 & -0.26342500 \\ \mathrm{C} & -0.62370600 & -2.60119300 & -0.74500500 \\ \mathrm{H} & -0.81360000 & -1.25375200 & -2.57306300 \\ \mathrm{H} & 1.76727800 & -1.96291200 & -3.07078000 \\ \mathrm{H} & 2.58850000 & -3.64383700 & -1.07837400 \\ \mathrm{H} & 0.50748600 & -3.98215500 & 0.65652000 \\ \mathrm{Fe} & 0.98062300 & -1.39516800 & -0.38441200 \\ \mathrm{C} & 1.16733900 & 0.65327900 & -0.33766800 \\ \mathrm{C} & 0.31526800 & 0.19576200 & 0.74180200 \\ \mathrm{C} & 2.46795900 & 0.03312100 & -0.19664900 \\ \mathrm{H} & 0.87245500 & 1.35648000 & -1.12770200 \\ \mathrm{C} & 1.09709200 & -0.72668500 & 1.54489900 \\ \mathrm{H} & -0.59095500 & 0.77448400 & 1.17421600 \\ \mathrm{C} & 2.41129100 & -0.82056700 & 0.97222500 \\ \mathrm{H} & 0.74235800 & -1.25496400 & 2.43952800 \\ \mathrm{H} & 3.23278200 & -1.44723100 & 1.34250500 \\ \mathrm{P} & -2.11497300 & -2.05301300 & 0.17424100 \\ \mathrm{P} & 3.85395500 & 0.33979400 & -1.37696500 \\ \mathrm{Ni} & -1.83150600 & 0.16831300 & 0.25179600 \\ \mathrm{O} & -3.36499000 & 1.02030100 & -0.45009100 \\ \mathrm{C} & -2.47616000 & 1.89581700 & -0.15088100 \\ \mathrm{C} & -2.30826700 & 3.24743700 & -0.17510400 \\ \mathrm{C} & -3.36503600 & 4.12632900 & -0.72191200 \\ \mathrm{C} & -3.15772600 & 5.53099600 & -0.83981800 \\ \mathrm{C} & -4.62260400 & 3.62685400 & -1.16866300\end{array}$




\begin{tabular}{|c|c|c|c|}
\hline $\mathrm{C}$ & -4.14316600 & 6.37899400 & -1.37084800 \\
\hline $\mathrm{H}$ & -2.20348900 & 5.97227900 & -0.51620800 \\
\hline $\mathrm{C}$ & -5.60378700 & 4.47765100 & -1.69639400 \\
\hline $\mathrm{H}$ & -4.82847600 & 2.54853300 & -1.09828700 \\
\hline $\mathrm{C}$ & -5.37704000 & 5.86349400 & -1.80401600 \\
\hline $\mathrm{H}$ & -3.93946300 & 7.46013300 & -1.44595300 \\
\hline $\mathrm{H}$ & -6.56372000 & 4.04976400 & -2.03022700 \\
\hline $\mathrm{C}$ & 4.66098300 & 1.83813700 & -0.61806700 \\
\hline $\mathrm{C}$ & 5.68102000 & 2.46489900 & -1.37358600 \\
\hline $\mathrm{C}$ & 4.28733100 & 2.40594500 & 0.62050700 \\
\hline $\mathrm{C}$ & 6.32653200 & 3.61538100 & -0.89177100 \\
\hline $\mathrm{H}$ & 5.97098400 & 2.04725500 & -2.35256200 \\
\hline $\mathrm{C}$ & 4.92645800 & 3.56616800 & 1.09596700 \\
\hline $\mathrm{H}$ & 3.48939800 & 1.94040400 & 1.21988800 \\
\hline $\mathrm{C}$ & 5.94886300 & 4.17135400 & 0.34478200 \\
\hline $\mathrm{H}$ & 7.12176200 & 4.08752600 & -1.49079200 \\
\hline $\mathrm{H}$ & 4.62226100 & 3.99827300 & 2.06297500 \\
\hline $\mathrm{H}$ & 6.44750300 & 5.07969800 & 0.71877800 \\
\hline $\mathrm{C}$ & 5.05495700 & -1.00589800 & -0.89677700 \\
\hline $\mathrm{C}$ & 5.85923400 & -0.96147300 & 0.26508800 \\
\hline $\mathrm{C}$ & 5.17970600 & -2.11238200 & -1.76811800 \\
\hline $\mathrm{C}$ & 6.75088000 & -2.00880700 & 0.55665300 \\
\hline $\mathrm{H}$ & 5.79375500 & -0.09560800 & 0.94319700 \\
\hline $\mathrm{C}$ & 6.06818000 & -3.16248400 & -1.47388100 \\
\hline $\mathrm{H}$ & 4.58242100 & -2.14461900 & -2.69430300 \\
\hline $\mathrm{C}$ & 6.85476900 & -3.11305000 & -0.30929400 \\
\hline $\mathrm{H}$ & 7.37261900 & -1.95975300 & 1.46520900 \\
\hline $\mathrm{H}$ & 6.15521900 & -4.01666200 & -2.16454500 \\
\hline $\mathrm{H}$ & 7.55740500 & -3.93037200 & -0.08117800 \\
\hline $\mathrm{C}$ & -2.09363900 & -3.05150400 & 1.73686800 \\
\hline $\mathrm{C}$ & -2.18902300 & -4.46158100 & 1.73538100 \\
\hline $\mathrm{C}$ & -2.00590500 & -2.36660800 & 2.96828300 \\
\hline $\mathrm{C}$ & -2.17631800 & -5.17201500 & 2.94740300 \\
\hline $\mathrm{H}$ & -2.28365400 & -5.00871000 & 0.78379900 \\
\hline $\mathrm{C}$ & -1.99782800 & -3.07950700 & 4.18088600 \\
\hline $\mathrm{H}$ & -1.95534700 & -1.26508000 & 2.97003300 \\
\hline $\mathrm{C}$ & -2.07924100 & -4.48273400 & 4.17116700 \\
\hline $\mathrm{H}$ & -2.25010700 & -6.27122500 & 2.93752300 \\
\hline $\mathrm{H}$ & -1.93368700 & -2.53479300 & 5.13623100 \\
\hline $\mathrm{H}$ & -2.07552000 & -5.04253200 & 5.11991700 \\
\hline $\mathrm{C}$ & -3.53914400 & -2.76978800 & -0.76538600 \\
\hline $\mathrm{C}$ & -3.38335200 & -3.80674100 & -1.71167500 \\
\hline $\mathrm{C}$ & -4.82591000 & -2.23614200 & -0.52556800 \\
\hline $\mathrm{C}$ & -4.50287400 & -4.31350400 & -2.39529300 \\
\hline $\mathrm{H}$ & -2.38151800 & -4.21372700 & -1.92357700 \\
\hline $\mathrm{C}$ & -5.94206900 & -2.75027200 & -1.20613200 \\
\hline $\mathrm{H}$ & -4.95087400 & -1.40581000 & 0.18787800 \\
\hline $\mathrm{C}$ & -5.78302500 & -3.78981200 & -2.14095400 \\
\hline $\mathrm{H}$ & -4.37252600 & -5.12099600 & -3.13355100 \\
\hline $\mathrm{H}$ & -6.94103000 & -2.32788200 & -1.01339200 \\
\hline $\mathrm{H}$ & -6.65833400 & -4.18640200 & -2.67957700 \\
\hline $\mathrm{C}$ & -1.03470500 & 3.84173300 & 0.39851800 \\
\hline $\mathrm{C}$ & -1.19479500 & 4.46751800 & 1.80191400 \\
\hline $\mathrm{H}$ & -0.62217600 & 4.60761000 & -0.29835000 \\
\hline $\mathrm{H}$ & -0.25807800 & 3.05055500 & 0.45108500 \\
\hline $\mathrm{C}$ & 0.08549000 & 5.13190300 & 2.33169700 \\
\hline $\mathrm{H}$ & -2.02053900 & 5.21383200 & 1.78742600 \\
\hline $\mathrm{H}$ & 0.91383900 & 4.38628900 & 2.32919500 \\
\hline $\mathrm{H}$ & 0.40385100 & 5.92912700 & 1.62122500 \\
\hline $\mathrm{H}$ & -6.15043400 & 6.52933700 & -2.21877400 \\
\hline $\mathrm{H}$ & -1.52618000 & 3.67324300 & 2.50930000 \\
\hline $\mathrm{C}$ & -0.06643900 & 5.72817300 & 3.7369970 \\
\hline
\end{tabular}




$\begin{array}{lrrr}\mathrm{H} & 0.87383800 & 6.20327700 & 4.08768300 \\ \mathrm{H} & -0.86196100 & 6.50367100 & 3.76267600 \\ \mathrm{H} & -0.34446600 & 4.94928600 & 4.47953900\end{array}$

\section{TS5 CC PD}

Electronic Contribution to Gibbs free energy in toluene at 373.15 K (BLYP/6-31g*): -5307.13174548

Energies and corrections (BP86/tzvp-svp):

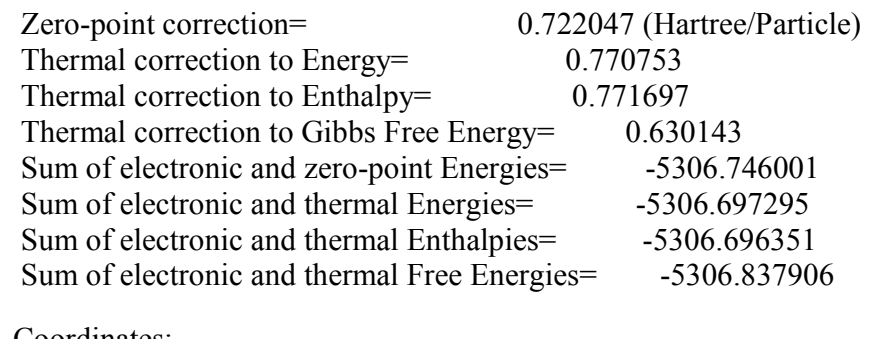

Coordinates:

$\begin{array}{lrrr}\mathrm{C} & 0.43688800 & -2.05137800 & 1.38584300 \\ \mathrm{C} & -0.03204600 & -3.40191400 & 1.53731600 \\ \mathrm{C} & 0.72497400 & -4.23861300 & 0.64163000 \\ \mathrm{C} & 1.66080500 & -3.40823400 & -0.07100000 \\ \mathrm{C} & 1.49331000 & -2.04345300 & 0.39183100 \\ \mathrm{H} & 0.06923100 & -1.16577400 & 1.92057000 \\ \mathrm{H} & -0.83930300 & -3.73128600 & 2.20429000 \\ \mathrm{H} & 0.59502100 & -5.32071900 & 0.50564000 \\ \mathrm{H} & 2.36768700 & -3.74529900 & -0.83928400 \\ \mathrm{Fe} & -0.24886900 & -2.76834400 & -0.40925600 \\ \mathrm{C} & -0.86331900 & -1.51024900 & -1.90410700 \\ \mathrm{C} & -0.49139100 & -2.78733000 & -2.45104100 \\ \mathrm{C} & -1.93455000 & -1.71056400 & -0.94736600 \\ \mathrm{H} & -0.41238400 & -0.54115600 & -2.15429300 \\ \mathrm{C} & -1.31682700 & -3.79108300 & -1.82895200 \\ \mathrm{H} & 0.29879300 & -2.96454600 & -3.19215800 \\ \mathrm{C} & -2.20293900 & -3.13410500 & -0.90254000 \\ \mathrm{H} & -1.26575300 & -4.87281000 & -2.01304800 \\ \mathrm{H} & -2.94489700 & -3.62387500 & -0.25909800 \\ \mathrm{P} & 2.39134100 & -0.51638700 & -0.08006700 \\ \mathrm{P} & -2.69954000 & -0.31010000 & -0.03103600 \\ \mathrm{Ni} & 1.25512500 & 1.28598600 & 0.26262600 \\ \mathrm{O} & 3.07085300 & 3.01203500 & -1.15062400 \\ \mathrm{C} & 2.07308100 & 2.78389200 & -0.50021100 \\ \mathrm{C} & 0.88064700 & 3.31720200 & 0.04292900 \\ \mathrm{C} & 0.47555600 & 2.89008000 & 1.39335100 \\ \mathrm{C} & -0.87937100 & 3.00183600 & 1.85744400 \\ \mathrm{C} & 1.42651400 & 2.27270300 & 2.29423300 \\ \mathrm{C} & -1.25035900 & 2.55417800 & 3.12765100 \\ \mathrm{H} & -1.63885300 & 3.45141800 & 1.20074100 \\ \mathrm{C} & 1.01610900 & 1.80416900 & 3.56578300 \\ \mathrm{H} & 2.50733300 & 2.34173000 & 2.08733500 \\ \mathrm{C} & -0.31230600 & 1.93811200 & 3.99065400 \\ \mathrm{H} & -2.29503500 & 2.67919700 & 3.45655900 \\ \mathrm{H} & 1.77107000 & 1.36226100 & 4.23624100 \\ \mathrm{C} & -4.12851400 & 0.13426600 & -1.14264600 \\ \mathrm{C} & -4.96642700 & 1.19799300 & -0.72763200 \\ \mathrm{C} & -4.37185000 & -0.46929800 & -2.39643700 \\ \mathrm{C} & -6.03096800 & 1.63049600 & -1.53446500 \\ \mathrm{H} & -4.78334200 & 1.69221400 & 0.24144600 \\ \mathrm{C} & -5.43215900 & -0.02637400 & -3.20958400\end{array}$




\begin{tabular}{|c|c|c|c|}
\hline $\mathrm{H}$ & -3.72900700 & -1.29399200 & -2.74177000 \\
\hline $\mathrm{C}$ & -6.26621500 & 1.02054000 & -2.78139700 \\
\hline $\mathrm{H}$ & -6.67673100 & 2.45503600 & -1.19161600 \\
\hline $\mathrm{H}$ & -5.60800800 & -0.50850600 & -4.18497000 \\
\hline $\mathrm{H}$ & -7.09631600 & 1.36468700 & -3.41871600 \\
\hline $\mathrm{C}$ & -3.58792700 & -1.18909600 & 1.35366500 \\
\hline $\mathrm{C}$ & -4.76489200 & -1.95040600 & 1.16547000 \\
\hline $\mathrm{C}$ & -3.06140700 & -1.06092300 & 2.65926600 \\
\hline $\mathrm{C}$ & -5.38488800 & -2.58738200 & 2.25448700 \\
\hline $\mathrm{H}$ & -5.20488100 & -2.03780700 & 0.15909600 \\
\hline $\mathrm{C}$ & -3.68032100 & -1.70201900 & 3.74819800 \\
\hline $\mathrm{H}$ & -2.16737900 & -0.43766800 & 2.82673100 \\
\hline $\mathrm{C}$ & -4.84172200 & -2.46856800 & 3.54746800 \\
\hline $\mathrm{H}$ & -6.30158700 & -3.17750100 & 2.09315000 \\
\hline $\mathrm{H}$ & -3.25843800 & -1.59132700 & 4.76010500 \\
\hline $\mathrm{H}$ & -5.33120100 & -2.96551100 & 4.40035500 \\
\hline $\mathrm{C}$ & 3.00873400 & -0.85405700 & -1.79993500 \\
\hline $\mathrm{C}$ & 4.02241200 & -1.80487500 & -2.05717200 \\
\hline $\mathrm{C}$ & 2.45329400 & -0.13103900 & -2.87596300 \\
\hline $\mathrm{C}$ & 4.44672300 & -2.04852900 & -3.37475600 \\
\hline $\mathrm{H}$ & 4.49560300 & -2.34730600 & -1.22299000 \\
\hline $\mathrm{C}$ & 2.87904200 & -0.37526600 & -4.19370100 \\
\hline $\mathrm{H}$ & 1.69601800 & 0.64222900 & -2.66505600 \\
\hline $\mathrm{C}$ & 3.87238200 & -1.33801500 & -4.44539100 \\
\hline $\mathrm{H}$ & 5.23764400 & -2.79151200 & -3.56597600 \\
\hline $\mathrm{H}$ & 2.44146400 & 0.19946100 & -5.02546200 \\
\hline $\mathrm{H}$ & 4.21118100 & -1.52596100 & -5.47676300 \\
\hline $\mathrm{C}$ & 3.95722500 & -0.59193200 & 0.91691400 \\
\hline $\mathrm{C}$ & 4.13972900 & -1.51555300 & 1.96838500 \\
\hline $\mathrm{C}$ & 4.96897200 & 0.36069800 & 0.64869200 \\
\hline $\mathrm{C}$ & 5.31903900 & -1.49146700 & 2.73600200 \\
\hline $\mathrm{H}$ & 3.35840900 & -2.25909000 & 2.18997300 \\
\hline $\mathrm{C}$ & 6.14525500 & 0.37772800 & 1.41589200 \\
\hline $\mathrm{H}$ & 4.83361000 & 1.09355600 & -0.16354400 \\
\hline $\mathrm{C}$ & 6.32385800 & -0.54772600 & 2.46172900 \\
\hline $\mathrm{H}$ & 5.45180100 & -2.21906000 & 3.55290400 \\
\hline $\mathrm{H}$ & 6.92736700 & 1.12206700 & 1.19636700 \\
\hline $\mathrm{H}$ & 7.24661500 & -0.53106900 & 3.06331300 \\
\hline $\mathrm{C}$ & -0.00627500 & 4.17751600 & -0.84694200 \\
\hline $\mathrm{C}$ & -0.04095300 & 5.65454500 & -0.40250100 \\
\hline $\mathrm{H}$ & -1.04143900 & 3.76722300 & -0.87193700 \\
\hline $\mathrm{H}$ & 0.36459500 & 4.11623600 & -1.89193200 \\
\hline $\mathrm{C}$ & -0.94885200 & 6.53120400 & -1.28025300 \\
\hline $\mathrm{H}$ & -0.37734600 & 5.71551500 & 0.65714600 \\
\hline $\mathrm{H}$ & -0.61061200 & 6.46208100 & -2.33947800 \\
\hline $\mathrm{H}$ & -1.98199400 & 6.11406500 & -1.26914600 \\
\hline $\mathrm{H}$ & -0.61823500 & 1.59055100 & 4.98984800 \\
\hline $\mathrm{H}$ & 0.99577200 & 6.05952000 & -0.41415300 \\
\hline $\mathrm{C}$ & -0.98337700 & 8.00220400 & -0.84647000 \\
\hline $\mathrm{H}$ & -1.64727200 & 8.60658000 & -1.49938000 \\
\hline $\mathrm{H}$ & -1.35298700 & 8.10813200 & 0.19614500 \\
\hline $\mathrm{H}$ & 0.02886300 & 8.45890900 & -0.88475800 \\
\hline
\end{tabular}

\section{9a CC PD}

Electronic Contribution to Gibbs free energy in toluene at 373.15 K (BLYP/6-31g*): -5307.13471787

Energies and corrections (BP86/tzvp-svp):

Zero-point correction $=$

Thermal correction to Energy=

Thermal correction to Enthalpy=
0.722161 (Hartree/Particle)

0.771621

0.772565 
Thermal correction to Gibbs Free Energy= Sum of electronic and zero-point Energies= Sum of electronic and thermal Energies= Sum of electronic and thermal Enthalpies= Sum of electronic and thermal Free Energies=
0.630636

$-5306.748415$

$-5306.698955$

$-5306.698011$

$-5306.839941$

Coordinates:

\begin{tabular}{|c|c|c|c|}
\hline & -0.09892200 & -1.45507500 & 1.73693900 \\
\hline $\mathrm{C}$ & -1.42813900 & -1.99021700 & 1.84696900 \\
\hline $\mathrm{C}$ & -1.66280000 & -2.85225800 & 0.71645700 \\
\hline $\mathrm{C}$ & -0.47520200 & -2.86254900 & -0.09566600 \\
\hline $\mathrm{C}$ & 0.50681900 & -2.00309400 & 0.53542800 \\
\hline $\mathrm{H}$ & 0.37969300 & -0.74766000 & 2.42750500 \\
\hline $\mathrm{H}$ & -2.14899000 & -1.75015200 & 2.63968100 \\
\hline $\mathrm{H}$ & -2.59515500 & -3.38911600 & 0.49719800 \\
\hline $\mathrm{H}$ & -0.33688500 & -3.40892100 & -1.03708300 \\
\hline $\mathrm{Fe}$ & -1.19124900 & -0.94960500 & 0.08512100 \\
\hline $\mathrm{C}$ & -1.57408600 & 1.05727000 & 0.19671300 \\
\hline $\mathrm{C}$ & -0.62274600 & 0.79409000 & -0.84840600 \\
\hline $\mathrm{C}$ & -2.79443400 & 0.33157400 & -0.10648300 \\
\hline $\mathrm{H}$ & -1.40265900 & 1.69381300 & 1.07510300 \\
\hline $\mathrm{C}$ & -1.22731800 & -0.11486600 & -1.79018200 \\
\hline $\mathrm{H}$ & 0.41012500 & 1.18541500 & -0.90235000 \\
\hline $\mathrm{C}$ & -2.56355700 & -0.39838900 & -1.33748400 \\
\hline $\mathrm{H}$ & -0.74680700 & -0.52994400 & -2.68593300 \\
\hline $\mathrm{H}$ & -3.28475100 & -1.06578300 & -1.82634800 \\
\hline $\mathrm{P}$ & 2.18180400 & -1.53055300 & -0.03988000 \\
\hline $\mathrm{P}$ & -4.28788400 & 0.39996600 & 0.97170900 \\
\hline $\mathrm{N}$ & 2.46364800 & 0.59783800 & 0.19117200 \\
\hline $\mathrm{O}$ & 5.08011100 & 0.76645900 & -0.99134500 \\
\hline $\mathrm{C}$ & 4.08912400 & 1.24038900 & -0.47888000 \\
\hline $\mathrm{C}$ & 3.40858800 & 2.42553200 & -0.11477000 \\
\hline $\mathrm{C}$ & 2.67034900 & 2.43781700 & 1.16115700 \\
\hline $\mathrm{C}$ & 1.58024100 & 3.34196700 & 1.40967100 \\
\hline $\mathrm{C}$ & 2.97260600 & 1.47501700 & 2.20055000 \\
\hline $\mathrm{C}$ & 0.86197900 & 3.29934200 & 2.60718700 \\
\hline $\mathrm{H}$ & 1.31118900 & 4.08610800 & 0.64471800 \\
\hline $\mathrm{C}$ & 2.21397900 & 1.44431200 & 3.39683800 \\
\hline $\mathrm{H}$ & 3.91343400 & 0.90146000 & 2.16212300 \\
\hline $\mathrm{C}$ & 1.16077100 & 2.34301100 & 3.60804200 \\
\hline $\mathrm{H}$ & 0.04756200 & 4.02414600 & 2.77101800 \\
\hline $\mathrm{H}$ & 2.48937600 & 0.71953800 & 4.18039600 \\
\hline $\mathrm{C}$ & -5.18915700 & 1.87300100 & 0.26906700 \\
\hline $\mathrm{C}$ & -6.36114900 & 2.29195000 & 0.94411900 \\
\hline $\mathrm{C}$ & -4.74329400 & 2.62157900 & -0.84308200 \\
\hline $\mathrm{C}$ & -7.08052000 & 3.41496400 & 0.50430900 \\
\hline $\mathrm{H}$ & -6.71359700 & 1.73118000 & 1.82640000 \\
\hline $\mathrm{C}$ & -5.45811500 & 3.75465400 & -1.27472800 \\
\hline $\mathrm{H}$ & -3.82956400 & 2.31786600 & -1.37749000 \\
\hline $\mathrm{C}$ & -6.62899400 & 4.15240000 & -0.60649400 \\
\hline $\mathrm{H}$ & -7.99399100 & 3.72283900 & 1.03827400 \\
\hline $\mathrm{H}$ & -5.09629400 & 4.32849400 & -2.14333900 \\
\hline $\mathrm{H}$ & -7.18738100 & 5.03909900 & -0.94663600 \\
\hline $\mathrm{C}$ & -5.30946100 & -1.00605900 & 0.29180200 \\
\hline $\mathrm{C}$ & -6.01709800 & -0.93978500 & -0.93043500 \\
\hline $\mathrm{C}$ & -5.39471900 & -2.18790600 & 1.06291600 \\
\hline $\mathrm{C}$ & -6.77414000 & -2.03678900 & -1.37788200 \\
\hline $\mathrm{H}$ & -5.98126600 & -0.01860600 & -1.53376900 \\
\hline $\mathrm{C}$ & -6.14827200 & -3.28731100 & 0.61313100 \\
\hline $\mathrm{H}$ & -4.87277400 & -2.24233500 & 2.03256900 \\
\hline $\mathrm{C}$ & -6.83860200 & -3.21404900 & -0.60973400 \\
\hline 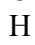 & -7.32091900 & -1.97028000 & -2.33241300 \\
\hline
\end{tabular}




$\begin{array}{lrrr}\mathrm{H} & -6.20537800 & -4.20069800 & 1.22690900 \\ \mathrm{H} & -7.43548500 & -4.07115200 & -0.96034000 \\ \mathrm{C} & 2.27082000 & -2.22382900 & -1.76082500 \\ \mathrm{C} & 2.36009400 & -3.61231000 & -2.00631900 \\ \mathrm{C} & 2.25220100 & -1.32824300 & -2.85046800 \\ \mathrm{C} & 2.40901600 & -4.09413100 & -3.32583100 \\ \mathrm{H} & 2.40259700 & -4.32082100 & -1.16357500 \\ \mathrm{C} & 2.30092200 & -1.81217800 & -4.16984600 \\ \mathrm{H} & 2.21547600 & -0.24449800 & -2.64803400 \\ \mathrm{C} & 2.37656200 & -3.19579200 & -4.40885500 \\ \mathrm{H} & 2.48034600 & -5.17816400 & -3.50972300 \\ \mathrm{H} & 2.29143700 & -1.10425900 & -5.01395900 \\ \mathrm{H} & 2.42148400 & -3.57617200 & -5.44184200 \\ \mathrm{C} & 3.33229800 & -2.63602100 & 0.90530600 \\ \mathrm{C} & 2.87265900 & -3.60589800 & 1.82202400 \\ \mathrm{C} & 4.72382700 & -2.46675700 & 0.71196000 \\ \mathrm{C} & 3.79246100 & -4.39925300 & 2.53261600 \\ \mathrm{H} & 1.79198800 & -3.74182600 & 1.98436500 \\ \mathrm{C} & 5.63646700 & -3.26553900 & 1.42015000 \\ \mathrm{H} & 5.09313300 & -1.70465700 & 0.00608400 \\ \mathrm{C} & 5.17372300 & -4.23252100 & 2.33272700 \\ \mathrm{H} & 3.42392500 & -5.15344600 & 3.24647600 \\ \mathrm{H} & 6.71792200 & -3.12770400 & 1.26123800 \\ \mathrm{H} & 5.89194300 & -4.85459700 & 2.89026800 \\ \mathrm{C} & 3.32299300 & 3.56136900 & -1.12512900 \\ \mathrm{C} & 4.11216400 & 4.81046400 & -0.68122800 \\ \mathrm{H} & 2.25945300 & 3.83703900 & -1.31060500 \\ \mathrm{H} & 3.71420100 & 3.20505600 & -2.10148600 \\ \mathrm{C} & 4.01484000 & 5.97644200 & -1.67830900 \\ \mathrm{H} & 3.74828400 & 5.14359100 & 0.31690700 \\ \mathrm{H} & 4.37448300 & 5.63549700 & -2.67607200 \\ \mathrm{H} & 2.94333600 & 6.24594800 & -1.82291700 \\ \mathrm{H} & 0.58599300 & 2.32193200 & 4.54688900 \\ \mathrm{H} & 5.17925100 & 4.52955400 & -0.53569900 \\ \mathrm{C} & 4.80313200 & 7.21880500 & -1.24438000 \\ \mathrm{H} & 4.71168100 & 8.04104300 & -1.98447200 \\ \mathrm{H} & 4.44216700 & 7.60631400 & -0.26749100 \\ \mathrm{H} & 5.88461400 & 6.99147200 & -1.12917600 \\ & & & \end{array}$

\section{TS2a Decarb PD}

Electronic Contribution to Gibbs free energy in toluene at 373.15 K (BLYP/6-31g*): -5307.09802342

Energies and corrections (BP86/tzvp-svp):

\begin{tabular}{|c|c|c|c|c|}
\hline \multicolumn{5}{|c|}{ Zero-point correction $=$} \\
\hline \multicolumn{3}{|c|}{ Thermal correction to Energy= } & \multicolumn{2}{|c|}{0.769728} \\
\hline \multicolumn{3}{|c|}{ Thermal correction to Enthalpy $=$} & \multicolumn{2}{|c|}{0.770673} \\
\hline \multicolumn{5}{|c|}{ Thermal correction to Gibbs Free Energy= } \\
\hline \multicolumn{5}{|c|}{ Sum of electronic and zero-point Energies $=$} \\
\hline \multicolumn{5}{|c|}{ Sum of electronic and thermal Energies $=$} \\
\hline \multicolumn{5}{|c|}{ Sum of electronic and thermal Enthalpies $=$} \\
\hline \multicolumn{5}{|c|}{ Sum of electronic and thermal Free Energies $=$} \\
\hline \multicolumn{5}{|c|}{ Coordina } \\
\hline $\mathrm{C}$ & 200 & -0.9 & & 9770 \\
\hline $\mathrm{C}$ & 1.63824000 & -1.35688800 & & 19( \\
\hline $\mathrm{C}$ & 2.02375400 & -2.39889300 & & 1 \\
\hline $\mathrm{C}$ & 0.90521900 & -2.67779500 & -0.37 & 50560 \\
\hline $\mathrm{C}$ & -0.18698300 & -1.80682300 & -0.76 & 2949( \\
\hline $\mathrm{H}$ & -0.30474500 & -0.21173600 & -2.38 & 0041 \\
\hline
\end{tabular}




\begin{tabular}{|c|c|c|c|}
\hline $\mathrm{H}$ & 2.27880300 & -0.89907900 & -2.91814100 \\
\hline $\mathrm{H}$ & 3.01062500 & -2.87689000 & -1.18013300 \\
\hline $\mathrm{H}$ & 0.88130200 & -3.40985200 & 0.44205300 \\
\hline $\mathrm{Fe}$ & 1.45541600 & -0.71033800 & -0.20567600 \\
\hline $\mathrm{C}$ & 1.78272100 & 1.29656500 & 0.04102400 \\
\hline $\mathrm{C}$ & 0.87244500 & 0.82307600 & 1.04739700 \\
\hline $\mathrm{C}$ & 3.03010100 & 0.56596100 & 0.17116100 \\
\hline $\mathrm{H}$ & 1.57600200 & 2.07966700 & -0.70064000 \\
\hline $\mathrm{C}$ & 1.53180100 & -0.22087200 & 1.79012300 \\
\hline $\mathrm{H}$ & -0.16076900 & 1.17233200 & 1.19599500 \\
\hline $\mathrm{C}$ & 2.85796000 & -0.37991100 & 1.25523800 \\
\hline $\mathrm{H}$ & 1.09267300 & -0.80553100 & 2.60915800 \\
\hline $\mathrm{H}$ & 3.61015200 & -1.10422100 & 1.59289200 \\
\hline $\mathrm{P}$ & -1.88675200 & -1.72431500 & -0.08689700 \\
\hline $\mathrm{P}$ & 4.48451400 & 0.87995500 & -0.91732900 \\
\hline $\mathrm{Ni}$ & -2.65848500 & 0.33062500 & 0.03296600 \\
\hline $\mathrm{O}$ & -5.08566500 & 0.50852000 & -1.49910500 \\
\hline $\mathrm{C}$ & -4.07723500 & 0.67168500 & -0.92766200 \\
\hline $\mathrm{C}$ & -3.18144700 & 2.14095100 & 0.14154400 \\
\hline $\mathrm{C}$ & -4.26815100 & 2.68557000 & 0.96381700 \\
\hline $\mathrm{C}$ & -5.12700000 & 1.84370400 & 1.73609400 \\
\hline $\mathrm{C}$ & -4.45705100 & 4.09315500 & 1.12084700 \\
\hline $\mathrm{C}$ & -6.11787900 & 2.36414400 & 2.57314100 \\
\hline $\mathrm{H}$ & -4.99131100 & 0.75093100 & 1.66894000 \\
\hline $\mathrm{C}$ & -5.43008800 & 4.61268500 & 1.99054900 \\
\hline $\mathrm{H}$ & -3.81374400 & 4.79540500 & 0.56989400 \\
\hline $\mathrm{C}$ & -6.27895500 & 3.75926800 & 2.71591700 \\
\hline $\mathrm{H}$ & -6.76560400 & 1.67453100 & 3.13927200 \\
\hline $\mathrm{H}$ & -5.53092000 & 5.70610600 & 2.09207000 \\
\hline $\mathrm{C}$ & 5.33447400 & 2.26801300 & -0.00828900 \\
\hline $\mathrm{C}$ & 6.45922100 & 2.85400400 & -0.63781100 \\
\hline $\mathrm{C}$ & 4.89184600 & 2.80149200 & 1.22278700 \\
\hline $\mathrm{C}$ & 7.13691300 & 3.92920500 & -0.04054600 \\
\hline $\mathrm{H}$ & 6.80720400 & 2.46334100 & -1.60908300 \\
\hline $\mathrm{C}$ & 5.56426600 & 3.88769200 & 1.81360000 \\
\hline $\mathrm{H}$ & 4.01358900 & 2.36675600 & 1.72513300 \\
\hline $\mathrm{C}$ & 6.68914000 & 4.45177600 & 1.18741900 \\
\hline $\mathrm{H}$ & 8.01400700 & 4.36911400 & -0.54203600 \\
\hline $\mathrm{H}$ & 5.20516200 & 4.29345600 & 2.77329500 \\
\hline $\mathrm{H}$ & 7.21416300 & 5.30164600 & 1.65193300 \\
\hline $\mathrm{C}$ & 5.59277900 & -0.56106500 & -0.49613100 \\
\hline $\mathrm{C}$ & 6.31909200 & -0.66227200 & 0.71268700 \\
\hline $\mathrm{C}$ & 5.72789200 & -1.58807000 & -1.45830000 \\
\hline $\mathrm{C}$ & 7.14402400 & -1.77372800 & 0.95909500 \\
\hline $\mathrm{H}$ & 6.24453400 & 0.13975400 & 1.46437200 \\
\hline $\mathrm{C}$ & 6.54966500 & -2.70254800 & -1.20983200 \\
\hline $\mathrm{H}$ & 5.19142700 & -1.50681600 & -2.41816900 \\
\hline $\mathrm{C}$ & 7.25840300 & -2.79792700 & 0.00082400 \\
\hline $\mathrm{H}$ & 7.70493600 & -1.83859000 & 1.90549000 \\
\hline $\mathrm{H}$ & 6.64535500 & -3.49371200 & -1.97085100 \\
\hline $\mathrm{H}$ & 7.90862000 & -3.66602100 & 0.19431600 \\
\hline $\mathrm{C}$ & -1.76793300 & -2.56646000 & 1.56361600 \\
\hline $\mathrm{C}$ & -1.67807300 & -3.97061400 & 1.69066200 \\
\hline $\mathrm{C}$ & -1.77836400 & -1.76597500 & 2.72710000 \\
\hline $\mathrm{C}$ & -1.57845600 & -4.55941400 & 2.96331900 \\
\hline $\mathrm{H}$ & -1.69661500 & -4.60832500 & 0.79261100 \\
\hline $\mathrm{C}$ & -1.68038500 & -2.35758600 & 3.99873500 \\
\hline $\mathrm{H}$ & -1.87529800 & -0.67141100 & 2.62727400 \\
\hline $\mathrm{C}$ & -1.57694200 & -3.75508800 & 4.11797500 \\
\hline $\mathrm{H}$ & -1.50834300 & -5.65521100 & 3.05383800 \\
\hline $\mathrm{H}$ & -1.69441600 & -1.72368300 & 4.89955700 \\
\hline $\mathrm{H}$ & -1.50477900 & -4.22038100 & 5.11391600 \\
\hline
\end{tabular}




$$
\begin{array}{rrr}
-2.82555300 & -2.95920400 & -1.10848500 \\
-2.21334900 & -3.73552800 & -2.11578300 \\
-4.21391200 & -3.09744200 & -0.87804900 \\
-2.97827400 & -4.63954700 & -2.87628400 \\
-1.13459400 & -3.63348400 & -2.31048700 \\
-4.97178200 & -4.00683000 & -1.63280000 \\
-4.70764100 & -2.48701100 & -0.10431100 \\
-4.35567100 & -4.77924200 & -2.63548900 \\
-2.49074900 & -5.23830700 & -3.66227700 \\
-6.05229200 & -4.10634600 & -1.44288500 \\
-4.95264700 & -5.48684200 & -3.23245500 \\
-2.48065600 & 3.03481200 & -0.87742700 \\
-3.31060400 & 3.63939500 & -2.03172200 \\
-1.60970000 & 2.49531800 & -1.31402400 \\
-2.01614000 & 3.86090600 & -0.28523800 \\
-2.53367100 & 4.66527000 & -2.87260100 \\
-3.65768900 & 2.81189700 & -2.69111200 \\
-2.20349900 & 5.50236100 & -2.21513500 \\
-1.59523000 & 4.19594900 & -3.24872700 \\
-7.04879000 & 4.16970400 & 3.38811900 \\
-4.23560800 & 4.10871600 & -1.63077300 \\
-3.33609000 & 5.22612800 & -4.05366800 \\
-2.74754000 & 5.96490300 & -4.63703100 \\
-3.64558500 & 4.41879700 & -4.75175500 \\
-4.26215200 & 5.73386300 & -3.70817000
\end{array}
$$

\begin{tabular}{|c|c|c|c|c|}
\hline \multicolumn{5}{|c|}{ Zero-point correction $=$} \\
\hline \multicolumn{3}{|c|}{ Thermal correction to Energy= } & \multicolumn{2}{|c|}{0.770395} \\
\hline \multicolumn{3}{|c|}{ Thermal correction to Enthalpy= } & \multicolumn{2}{|c|}{0.771340} \\
\hline \multicolumn{5}{|c|}{ Thermal correction to Gibbs Free Energy= } \\
\hline \multicolumn{5}{|c|}{ Sum of electronic and zero-point Energies= } \\
\hline \multicolumn{5}{|c|}{ Sum of electronic and thermal Energies $=$} \\
\hline \multicolumn{5}{|c|}{ Sum of electronic and thermal Enthalpies $=$} \\
\hline \multicolumn{5}{|c|}{ Sum of electronic and thermal Free Energies $=$} \\
\hline \multicolumn{5}{|c|}{ Coordinates: } \\
\hline $\mathrm{C}$ & 0.26148300 & -2.97199700 & -0.98 & 9100 \\
\hline $\mathrm{C}$ & 0.82391100 & -4.25632900 & -0. & 110 \\
\hline $\mathrm{C}$ & 0.19982600 & -4.73197500 & & \\
\hline $\mathrm{C}$ & -0.75252900 & -3.74375400 & 0 . & 620 \\
\hline $\mathrm{C}$ & -0.72670500 & -2.64391000 & 0.02 & 130 \\
\hline $\mathrm{H}$ & 0.53142700 & -2.33715800 & -1.83 & 429300 \\
\hline $\mathrm{H}$ & 1.61091800 & -4.76954300 & -1.2 & 3470 \\
\hline $\mathrm{H}$ & 0.42793400 & -5.67425900 & 1. & 7700 \\
\hline $\mathrm{H}$ & -1.37849600 & -3.80374400 & 1.87 & 81500 \\
\hline $\mathrm{Fe}$ & 1.11614400 & -2.92250600 & $0.87^{\circ}$ & 798700 \\
\hline $\mathrm{C}$ & 1.49645400 & -1.36930500 & 2.148 & 59500 \\
\hline $\mathrm{C}$ & 1.72935100 & -2.62052000 & 2.8 & 33500 \\
\hline $\mathrm{C}$ & 2.36812100 & -1.29831500 & 0.985 & 3500 \\
\hline $\mathrm{H}$ & 0.77457700 & -0.60150600 & 2.45 & 80500 \\
\hline $\mathrm{C}$ & 2.73155900 & -3.34096400 & 2.077 & 00000 \\
\hline $\mathrm{H}$ & 1.21160100 & -2.97271100 & 3.72 & 31800 \\
\hline $\mathrm{C}$ & 3.12653500 & -2.53304400 & 0.952 & 86400 \\
\hline $\mathrm{H}$ & 3.11305800 & -4.34323500 & 2.31 & 48000 \\
\hline $\mathrm{H}$ & 3.86173800 & -2.80825100 & 0.18 & 641700 \\
\hline
\end{tabular}

\section{TS6 Carb2 PD}

Electronic Contribution to Gibbs free energy in toluene at 373.15 K (BLYP/6-31g*): -5307.13468589

Energies and corrections (BP86/tzvp-svp): 


\begin{tabular}{|c|c|c|c|}
\hline $\mathrm{P}$ & -1.72628900 & -1.10169800 & 0.00012100 \\
\hline $\mathrm{P}$ & 2.37940500 & 0.13437000 & -0.16730200 \\
\hline $\mathrm{Ni}$ & -0.90142500 & 0.76723200 & -0.88210800 \\
\hline $\mathrm{O}$ & -0.72120700 & 0.28815700 & -3.75647500 \\
\hline $\mathrm{C}$ & -0.70769300 & 0.40497600 & -2.59164600 \\
\hline $\mathrm{C}$ & -1.18441700 & 2.42646700 & -0.16367000 \\
\hline $\mathrm{C}$ & -2.50418700 & 3.07566900 & -0.27593800 \\
\hline $\mathrm{C}$ & -3.25187200 & 2.99533400 & -1.48672700 \\
\hline $\mathrm{C}$ & -3.10531500 & 3.77352900 & 0.81315600 \\
\hline $\mathrm{C}$ & -4.51430700 & 3.59230900 & -1.60718300 \\
\hline $\mathrm{H}$ & -2.80084200 & 2.46777800 & -2.34097300 \\
\hline $\mathrm{C}$ & -4.38660300 & 4.33468600 & 0.70268000 \\
\hline $\mathrm{H}$ & -2.57106900 & 3.84517000 & 1.77356000 \\
\hline $\mathrm{C}$ & -5.09592800 & 4.25772300 & -0.51014200 \\
\hline $\mathrm{H}$ & -5.05536100 & 3.53160000 & -2.56543200 \\
\hline $\mathrm{H}$ & -4.83450700 & 4.84487700 & 1.57122600 \\
\hline $\mathrm{C}$ & 3.58438200 & 1.28371600 & 0.67635900 \\
\hline $\mathrm{C}$ & 4.00966600 & 2.42944700 & -0.03971100 \\
\hline $\mathrm{C}$ & 4.03156900 & 1.11302000 & 2.00563000 \\
\hline $\mathrm{C}$ & 4.87406100 & 3.36530100 & 0.55091500 \\
\hline $\mathrm{H}$ & 3.66655400 & 2.58655700 & -1.07599000 \\
\hline $\mathrm{C}$ & 4.88843300 & 2.05873200 & 2.59980200 \\
\hline $\mathrm{H}$ & 3.71523400 & 0.22995000 & 2.58222200 \\
\hline $\mathrm{C}$ & 5.31541600 & 3.18482500 & 1.87582400 \\
\hline $\mathrm{H}$ & 5.20300500 & 4.24425300 & -0.02651000 \\
\hline $\mathrm{H}$ & 5.22972000 & 1.90685800 & 3.63675100 \\
\hline $\mathrm{H}$ & 5.98961000 & 3.92148200 & 2.34098000 \\
\hline $\mathrm{C}$ & 3.45575800 & -0.50262800 & -1.55255500 \\
\hline $\mathrm{C}$ & 4.84125900 & -0.74509100 & -1.39752700 \\
\hline $\mathrm{C}$ & 2.86338400 & -0.72918100 & -2.81432900 \\
\hline $\mathrm{C}$ & 5.60705900 & -1.22239400 & -2.47470600 \\
\hline $\mathrm{H}$ & 5.32630200 & -0.55042900 & -0.42774200 \\
\hline $\mathrm{C}$ & 3.63025100 & -1.20824700 & -3.89273400 \\
\hline $\mathrm{H}$ & 1.79389900 & -0.51210000 & -2.95855700 \\
\hline $\mathrm{C}$ & 5.00277100 & -1.45822400 & -3.72421100 \\
\hline $\mathrm{H}$ & 6.68511700 & -1.40692400 & -2.33923100 \\
\hline $\mathrm{H}$ & 3.15136600 & -1.37583300 & -4.87063500 \\
\hline $\mathrm{H}$ & 5.60605000 & -1.82798500 & -4.56877800 \\
\hline $\mathrm{C}$ & -2.40236500 & -1.00662600 & 1.73388100 \\
\hline $\mathrm{C}$ & -3.35205700 & -1.93605400 & 2.21693000 \\
\hline $\mathrm{C}$ & -1.96568100 & 0.03018500 & 2.58435000 \\
\hline $\mathrm{C}$ & -3.83270500 & -1.84071100 & 3.53448400 \\
\hline $\mathrm{H}$ & -3.73123100 & -2.73079900 & 1.55490900 \\
\hline $\mathrm{C}$ & -2.44239600 & 0.12188300 & 3.90451100 \\
\hline $\mathrm{H}$ & -1.26237700 & 0.78149500 & 2.18843800 \\
\hline $\mathrm{C}$ & -3.37471900 & -0.81546800 & 4.38272100 \\
\hline $\mathrm{H}$ & -4.57600400 & -2.56809200 & 3.89870100 \\
\hline $\mathrm{H}$ & -2.09226400 & 0.93681300 & 4.55814600 \\
\hline $\mathrm{H}$ & -3.75571100 & -0.74055900 & 5.41376600 \\
\hline $\mathrm{C}$ & -3.23993000 & -1.60204000 & -0.95604300 \\
\hline $\mathrm{C}$ & -3.32427000 & -2.81682700 & -1.66956200 \\
\hline $\mathrm{C}$ & -4.32182600 & -0.69187100 & -1.01409500 \\
\hline $\mathrm{C}$ & -4.47402000 & -3.11895400 & -2.42299500 \\
\hline $\mathrm{H}$ & -2.48911200 & -3.53337800 & -1.63805200 \\
\hline $\mathrm{C}$ & -5.46915000 & -1.00028500 & -1.76255600 \\
\hline $\mathrm{H}$ & -4.26572900 & 0.26603900 & -0.47201400 \\
\hline $\mathrm{C}$ & -5.54849700 & -2.21444100 & -2.47052200 \\
\hline $\mathrm{H}$ & -4.52764300 & -4.07099600 & -2.97529900 \\
\hline $\mathrm{H}$ & -6.30508500 & -0.28343300 & -1.79700900 \\
\hline $\mathrm{H}$ & -6.44751700 & -2.45319500 & -3.06085000 \\
\hline $\mathrm{C}$ & -0.14224000 & 3.23455400 & 0.57372700 \\
\hline $\mathrm{C}$ & 0.26702800 & 4.51695200 & -0.1963660 \\
\hline
\end{tabular}




$\begin{array}{lrrr}\mathrm{H} & 0.76427100 & 2.62721900 & 0.77539600 \\ \mathrm{H} & -0.54327900 & 3.55210400 & 1.56905800 \\ \mathrm{C} & 1.27982300 & 5.38740100 & 0.56405800 \\ \mathrm{H} & 0.69138600 & 4.21977900 & -1.18163300 \\ \mathrm{H} & 0.84423800 & 5.67879700 & 1.54731500 \\ \mathrm{H} & 2.18170400 & 4.77964100 & 0.80120900 \\ \mathrm{H} & -6.09592000 & 4.71132800 & -0.59963100 \\ \mathrm{H} & -0.64341700 & 5.11521200 & -0.42288600 \\ \mathrm{C} & 1.69457900 & 6.64669100 & -0.20783100 \\ \mathrm{H} & 2.42246700 & 7.25763000 & 0.36625600 \\ \mathrm{H} & 2.16819900 & 6.38832700 & -1.17939000 \\ \mathrm{H} & 0.81865000 & 7.29306400 & -0.43139700\end{array}$

\section{0a Carb2 PD Cis}

Electronic Contribution to Gibbs free energy in toluene at 373.15 K (BLYP/6-31g*): -5307.13483238

Energies and corrections (BP86/tzvp-svp):

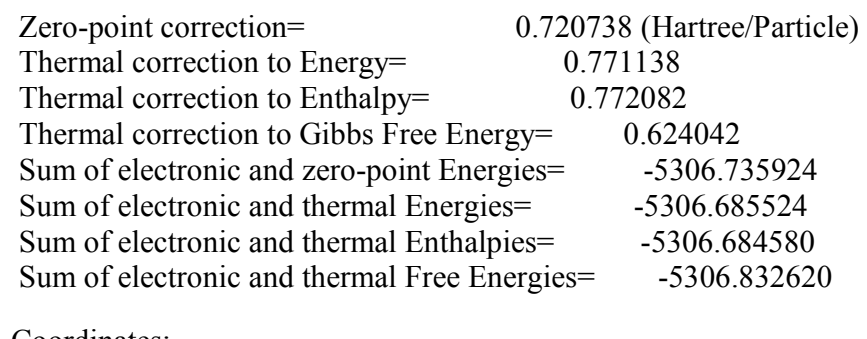

Coordinates:

$\begin{array}{lrrr}\mathrm{C} & 0.50245400 & -1.89982700 & -1.55268200 \\ \mathrm{C} & 1.80497600 & -2.46405000 & -1.32779700 \\ \mathrm{C} & 1.89629900 & -2.85267900 & 0.05643200 \\ \mathrm{C} & 0.64527600 & -2.53793900 & 0.69335200 \\ \mathrm{C} & -0.23268900 & -1.95006900 & -0.30184200 \\ \mathrm{H} & 0.12596700 & -1.50022900 & -2.50261100 \\ \mathrm{H} & 2.60119300 & -2.54777800 & -2.07930600 \\ \mathrm{H} & 2.77571700 & -3.28932700 & 0.54791800 \\ \mathrm{H} & 0.39654300 & -2.69882700 & 1.74977200 \\ \mathrm{Fe} & 1.47770600 & -0.84366500 & -0.09844000 \\ \mathrm{C} & 1.95292000 & 0.97919500 & -0.89048200 \\ \mathrm{C} & 0.91617500 & 1.12163300 & 0.09553200 \\ \mathrm{C} & 3.11714500 & 0.39613400 & -0.24797500 \\ \mathrm{H} & 1.87879100 & 1.25734800 & -1.95030700 \\ \mathrm{C} & 1.41575200 & 0.61205500 & 1.34793100 \\ \mathrm{H} & -0.10098700 & 1.49615000 & -0.09135300 \\ \mathrm{C} & 2.76756700 & 0.16444400 & 1.13954300 \\ \mathrm{H} & 0.85575400 & 0.55379300 & 2.29069800 \\ \mathrm{H} & 3.42319600 & -0.28997300 & 1.89330100 \\ \mathrm{P} & -1.96544200 & -1.37968900 & -0.10374600 \\ \mathrm{P} & 4.68696400 & 0.05358400 & -1.15058100 \\ \mathrm{Ni} & -2.54569400 & 0.50922900 & -1.14570200 \\ \mathrm{O} & -2.14622300 & 0.03551100 & -3.99977000 \\ \mathrm{C} & -2.29055500 & 0.16545000 & -2.84856200 \\ \mathrm{C} & -3.24090400 & 2.08124500 & -0.53439000 \\ \mathrm{C} & -4.70213600 & 2.19296700 & -0.36968600 \\ \mathrm{C} & -5.46562700 & 3.23262800 & -0.97762900 \\ \mathrm{C} & -5.41320700 & 1.22009100 & 0.39038900 \\ \mathrm{C} & -6.86177400 & 3.27617400 & -0.85366800 \\ \mathrm{H} & -4.95559700 & 3.99378300 & -1.58906100 \\ \mathrm{C} & -6.80598600 & 1.28744200 & 0.54317800 \\ \mathrm{H} & -4.83706400 & 0.42403400 & 0.88780900\end{array}$




\begin{tabular}{|c|c|c|c|}
\hline $\mathrm{C}$ & -7.54048500 & 2.30982900 & -0.08658200 \\
\hline $\mathrm{H}$ & -7.42897400 & 4.07589300 & -1.35745000 \\
\hline $\mathrm{H}$ & -7.32498200 & 0.53094300 & 1.15387700 \\
\hline $\mathrm{C}$ & 5.58642600 & 1.67620500 & -0.96487600 \\
\hline $\mathrm{C}$ & 6.81748100 & 1.81218800 & -1.65099900 \\
\hline $\mathrm{C}$ & 5.08786100 & 2.78269300 & -0.24159400 \\
\hline $\mathrm{C}$ & 7.54146800 & 3.01420800 & -1.59552100 \\
\hline $\mathrm{H}$ & 7.21268100 & 0.96530700 & -2.23726200 \\
\hline $\mathrm{C}$ & 5.80815300 & 3.99112200 & -0.19776600 \\
\hline $\mathrm{H}$ & 4.12802500 & 2.70082000 & 0.29198300 \\
\hline $\mathrm{C}$ & 7.03692500 & 4.10983000 & -0.86986800 \\
\hline $\mathrm{H}$ & 8.50076300 & 3.10003300 & -2.13099400 \\
\hline $\mathrm{H}$ & 5.40460000 & 4.84544900 & 0.36967600 \\
\hline $\mathrm{H}$ & 7.59936100 & 5.05646500 & -0.83380500 \\
\hline $\mathrm{C}$ & 5.60388000 & -1.01027200 & 0.07864300 \\
\hline $\mathrm{C}$ & 6.22964600 & -0.50234600 & 1.24025500 \\
\hline $\mathrm{C}$ & 5.69414600 & -2.39516000 & -0.19098400 \\
\hline $\mathrm{C}$ & 6.91200500 & -1.36262900 & 2.11837400 \\
\hline $\mathrm{H}$ & 6.18862000 & 0.57737600 & 1.45549300 \\
\hline $\mathrm{C}$ & 6.37228700 & -3.25681300 & 0.69012700 \\
\hline $\mathrm{H}$ & 5.23675300 & -2.80072800 & -1.10851600 \\
\hline $\mathrm{C}$ & 6.98200700 & -2.74177300 & 1.84783700 \\
\hline $\mathrm{H}$ & 7.39585300 & -0.95195600 & 3.01937300 \\
\hline $\mathrm{H}$ & 6.43440200 & -4.33357900 & 0.46430100 \\
\hline $\mathrm{H}$ & 7.52056300 & -3.41360100 & 2.53519300 \\
\hline $\mathrm{C}$ & -2.20261900 & -1.44862700 & 1.74288400 \\
\hline $\mathrm{C}$ & -2.36312500 & -2.66995200 & 2.43599900 \\
\hline $\mathrm{C}$ & -2.21912400 & -0.23894900 & 2.46903500 \\
\hline $\mathrm{C}$ & -2.52109800 & -2.67660100 & 3.83299400 \\
\hline $\mathrm{H}$ & -2.37441100 & -3.62194000 & 1.88197000 \\
\hline $\mathrm{C}$ & -2.37039100 & -0.24775200 & 3.86727000 \\
\hline $\mathrm{H}$ & -2.13423400 & 0.71311900 & 1.91855300 \\
\hline $\mathrm{C}$ & -2.52087400 & -1.46688000 & 4.55172000 \\
\hline $\mathrm{H}$ & -2.65039100 & -3.63397300 & 4.36291600 \\
\hline $\mathrm{H}$ & -2.38332900 & 0.70380600 & 4.42250100 \\
\hline $\mathrm{H}$ & -2.64892200 & -1.47478200 & 5.64593400 \\
\hline $\mathrm{C}$ & -2.96141200 & -2.83431800 & -0.68977400 \\
\hline $\mathrm{C}$ & -2.38633000 & -4.09359000 & -0.96882300 \\
\hline $\mathrm{C}$ & -4.34948200 & -2.65166400 & -0.89155500 \\
\hline $\mathrm{C}$ & -3.18853300 & -5.15339100 & -1.43044300 \\
\hline $\mathrm{H}$ & -1.30477900 & -4.24718100 & -0.83050100 \\
\hline $\mathrm{C}$ & -5.14809900 & -3.71400900 & -1.34551400 \\
\hline $\mathrm{H}$ & -4.80731700 & -1.66730000 & -0.69923200 \\
\hline $\mathrm{C}$ & -4.56935300 & -4.96779100 & -1.61678900 \\
\hline $\mathrm{H}$ & -2.72785500 & -6.13054800 & -1.64744500 \\
\hline $\mathrm{H}$ & -6.22791400 & -3.55806000 & -1.49839900 \\
\hline $\mathrm{H}$ & -5.19496100 & -5.79838000 & -1.98058500 \\
\hline $\mathrm{C}$ & -2.47877500 & 3.38274300 & -0.43762600 \\
\hline $\mathrm{C}$ & -2.56876800 & 4.06342300 & 0.95061700 \\
\hline $\mathrm{H}$ & -2.88929500 & 4.09643400 & -1.19600300 \\
\hline $\mathrm{H}$ & -1.41064800 & 3.24120900 & -0.70603100 \\
\hline $\mathrm{C}$ & -1.78308600 & 5.38198600 & 1.03160400 \\
\hline $\mathrm{H}$ & -3.63644300 & 4.24592600 & 1.20383700 \\
\hline $\mathrm{H}$ & -0.71879300 & 5.19238800 & 0.76362900 \\
\hline $\mathrm{H}$ & -2.16699600 & 6.08251300 & 0.25521900 \\
\hline $\mathrm{H}$ & -8.63627000 & 2.35341400 & 0.01800000 \\
\hline $\mathrm{H}$ & -2.18830500 & 3.35893900 & 1.72516000 \\
\hline $\mathrm{C}$ & -1.85571300 & 6.05028700 & 2.41039800 \\
\hline $\mathrm{H}$ & -1.28144200 & 6.99985900 & 2.43526300 \\
\hline $\mathrm{H}$ & -2.90448600 & 6.28633700 & 2.69153200 \\
\hline $\mathrm{H}$ & -1.44257800 & 5.38983900 & 3.20298500 \\
\hline
\end{tabular}




\section{0a Carb2 PD Trans}

Electronic Contribution to Gibbs free energy in toluene at 373.15 K (BLYP/6-31g*): -5307.13719722

Energies and corrections (BP86/tzvp-svp):

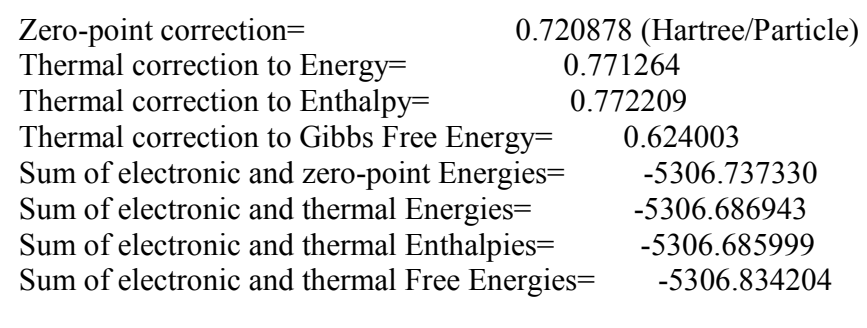

Coordinates:

$\begin{array}{lccc}\mathrm{C} & 0.55539300 & -1.59318800 & -1.75100200 \\ \mathrm{C} & 1.87565700 & -2.15665900 & -1.67885400 \\ \mathrm{C} & 2.03661900 & -2.76436000 & -0.38277900 \\ \mathrm{C} & 0.81276400 & -2.58586000 & 0.35076600 \\ \mathrm{C} & -0.12014000 & -1.86335500 & -0.49428300 \\ \mathrm{H} & 0.13081800 & -1.04893200 & -2.60342700 \\ \mathrm{H} & 2.63765900 & -2.09790800 & -2.46730500 \\ \mathrm{H} & 2.94444300 & -3.25459500 & -0.00727300 \\ \mathrm{H} & 0.61944200 & -2.92002600 & 1.37758800 \\ \mathrm{Fe} & 1.58164400 & -0.76502600 & -0.18886000 \\ \mathrm{C} & 2.04917500 & 1.15683400 & -0.70795600 \\ \mathrm{C} & 1.01444600 & 1.15118300 & 0.28989200 \\ \mathrm{C} & 3.21655300 & 0.49098300 & -0.15867000 \\ \mathrm{H} & 1.97146800 & 1.58489100 & -1.71629100 \\ \mathrm{C} & 1.51754300 & 0.46565700 & 1.45385700 \\ \mathrm{H} & -0.00227000 & 1.55029500 & 0.16149100 \\ \mathrm{C} & 2.87053900 & 0.05830300 & 1.18061200 \\ \mathrm{H} & 0.95882300 & 0.26755600 & 2.37815300 \\ \mathrm{H} & 3.52911500 & -0.49913100 & 1.85892000 \\ \mathrm{P} & -1.84144600 & -1.34981200 & -0.11587400 \\ \mathrm{P} & 4.78473500 & 0.28957700 & -1.10553900 \\ \mathrm{Ni} & -2.55832600 & 0.62834400 & -0.85064400 \\ \mathrm{O} & -1.74359800 & 0.94997300 & -3.63718100 \\ \mathrm{C} & -2.03100400 & 0.76520800 & -2.51949600 \\ \mathrm{C} & -3.58825500 & 1.82202400 & 0.07718800 \\ \mathrm{C} & -5.05882100 & 1.75462600 & 0.09207700 \\ \mathrm{C} & -5.75630800 & 1.20628900 & -1.02487100 \\ \mathrm{C} & -5.84642400 & 2.18239400 & 1.20161500 \\ \mathrm{C} & -7.15311800 & 1.10642700 & -1.03987300 \\ \mathrm{H} & -5.16218100 & 0.88070500 & -1.89392900 \\ \mathrm{C} & -7.24331000 & 2.05229300 & 1.19851900 \\ \mathrm{H} & -5.35100000 & 2.59450200 & 2.09455400 \\ \mathrm{H} & -7.90640800 & 1.52221700 & 0.07625300 \\ \mathrm{H} & -7.66214400 & 0.69592100 & -1.92686100 \\ \mathrm{H} & -7.82306600 & 2.37302900 & 2.07943900 \\ \mathrm{H} & 5.68675400 & 1.86330100 & -0.67405000 \\ \mathrm{C} & 6.91799800 & 2.10066400 & -1.33172800 \\ \mathrm{C} & 5.18974100 & 2.84725900 & 0.20957700 \\ \mathrm{C} & 7.64378300 & 3.27905700 & -1.09365400 \\ \mathrm{C} & 7.31187300 & 1.35264700 & -2.04055800 \\ \mathrm{H} & 5.91188100 & 4.03378500 & 0.43709200 \\ \mathrm{H} & 4.22967200 & 2.68654200 & 0.72449200 \\ \mathrm{H} & 7.14087400 & 4.25185100 & -0.20917000 \\ \mathrm{H} & & & \end{array}$




\begin{tabular}{rrr}
5.70383400 & -0.95144200 & -0.05735700 \\
6.32583600 & -0.63103800 & 1.17120700 \\
5.80089500 & -2.27579100 & -0.54266200 \\
7.01055300 & -1.61665300 & 1.90372800 \\
6.28029900 & 0.40095800 & 1.55429500 \\
6.48167400 & -3.26320600 & 0.19244100 \\
5.34652500 & -2.53260600 & -1.51383300 \\
7.08711600 & -2.93530100 & 1.41852600 \\
7.49118000 & -1.35174000 & 2.85944300 \\
6.54910300 & -4.29039800 & -0.20077400 \\
7.62748600 & -3.70521400 & 1.99218500 \\
-1.96588100 & -1.68020500 & 1.71113600 \\
-2.02339200 & -2.99313900 & 2.23141000 \\
-2.00169800 & -0.58618000 & 2.60090800 \\
-2.09931400 & -3.20297500 & 3.61940500 \\
-2.01950400 & -3.85745000 & 1.54833200 \\
-2.07000200 & -0.79749500 & 3.98965100 \\
-1.99679100 & 0.43678700 & 2.19129900 \\
-2.11786600 & -2.10649300 & 4.50111100 \\
-2.14932200 & -4.23034900 & 4.01449600 \\
-2.09798800 & 0.06523000 & 4.67438200 \\
-2.18033300 & -2.27344000 & 5.58830100 \\
-2.88120200 & -2.71140400 & -0.83102000 \\
-2.34225000 & -3.75742200 & -1.61056200 \\
-4.28023800 & -2.65486200 & -0.62376600 \\
-3.18810000 & -4.73459500 & -2.16735700 \\
-1.25645000 & -3.81098700 & -1.78445500 \\
-5.11935900 & -3.63540800 & -1.17701900 \\
-4.71592600 & -1.83831400 & -0.02481900 \\
-4.57574400 & -4.67755200 & -1.95169700 \\
-2.75564800 & -5.54699200 & -2.77327900 \\
-6.20639300 & -3.58196500 & -1.00581300 \\
-5.23563400 & -5.44364200 & -2.38902700 \\
-2.97392600 & 2.97959700 & 0.83366400 \\
-3.21387700 & 4.34128300 & 0.13174600 \\
-1.87903700 & 2.83596900 & 0.95700000 \\
-3.39542800 & 3.04406200 & 1.86715200 \\
-2.55196300 & 5.52363700 & 0.85728400 \\
-2.82708700 & 4.27722000 & -0.90987200 \\
-2.94045000 & 5.57794100 & 1.90013100 \\
-1.45937800 & 5.32945800 & 0.95377500 \\
-9.00436200 & 1.43151200 & 0.07142600 \\
-4.30822000 & 4.52040800 & 0.04135200 \\
-2.77470300 & 6.86841300 & 0.15348800 \\
-2.28543000 & 7.70172900 & 0.69977800 \\
-3.85640600 & 7.85764000 & -0.87839900 \\
& & \\
\hline
\end{tabular}

TS7-Cis Hydrogen Migration TS with one Phosphine Dissociated

Electronic Contribution to Gibbs free energy in toluene at 373.15 K (BLYP/6-31g*): -5307.08403873

Energies and corrections (BP86/tzvp-svp):

Zero-point correction=

Thermal correction to Energy=

Thermal correction to Enthalpy=

Thermal correction to Gibbs Free Energy=

Sum of electronic and zero-point Energies=

Sum of electronic and thermal Energies $=$

Sum of electronic and thermal Enthalpies=

Sum of electronic and thermal Free Energies=
0.716022 (Hartree/Particle)

0.766451

0.767395

0.620102

$-5306.701433$

$-5306.651004$

$-5306.650060$

$-5306.797353$ 
Coordinates:

\begin{tabular}{|c|c|c|c|}
\hline $\mathrm{C}$ & 0.69155000 & -1.77375800 & -1.52017300 \\
\hline $\mathrm{C}$ & 2.01175900 & -2.30697000 & -1.32171800 \\
\hline $\mathrm{C}$ & 2.13186700 & -2.71514600 & 0.05509000 \\
\hline $\mathrm{C}$ & 0.87935900 & -2.44654800 & 0.71060900 \\
\hline $\mathrm{C}$ & -0.02549600 & -1.86456800 & -0.26211800 \\
\hline $\mathrm{H}$ & 0.29139700 & -1.36479900 & -2.45654000 \\
\hline $\mathrm{H}$ & 2.80014700 & -2.35687100 & -2.08451600 \\
\hline $\mathrm{H}$ & 3.02898700 & -3.13603500 & 0.52814500 \\
\hline $\mathrm{H}$ & 0.64585100 & -2.63421200 & 1.76629700 \\
\hline $\mathrm{Fe}$ & 1.66117300 & -0.71540200 & -0.06426600 \\
\hline $\mathrm{C}$ & 2.06797400 & 1.12682700 & -0.84619500 \\
\hline $\mathrm{C}$ & 1.04449800 & 1.23063200 & 0.15848600 \\
\hline $\mathrm{C}$ & 3.26035300 & 0.57329600 & -0.22880100 \\
\hline $\mathrm{H}$ & 1.96524400 & 1.40990000 & -1.90223400 \\
\hline $\mathrm{C}$ & 1.58132100 & 0.72424600 & 1.39717000 \\
\hline $\mathrm{H}$ & 0.01036900 & 1.56787400 & -0.01915000 \\
\hline $\mathrm{C}$ & 2.94222800 & 0.31868800 & 1.16206600 \\
\hline $\mathrm{H}$ & 1.04396300 & 0.63862000 & 2.35079100 \\
\hline $\mathrm{H}$ & 3.62386000 & -0.12163700 & 1.90096300 \\
\hline $\mathrm{P}$ & -1.77702100 & -1.34556900 & -0.03936700 \\
\hline $\mathrm{P}$ & 4.82446400 & 0.28722000 & -1.15933700 \\
\hline $\mathrm{Ni}$ & -2.36243500 & 0.59640600 & -0.94853300 \\
\hline $\mathrm{O}$ & -1.33869700 & 1.04802400 & -3.64650500 \\
\hline $\mathrm{C}$ & -1.75450500 & 0.85132900 & -2.56896000 \\
\hline $\mathrm{C}$ & -3.47286700 & 1.91768200 & -0.15603300 \\
\hline $\mathrm{C}$ & -4.88376800 & 1.64492800 & -0.55833400 \\
\hline $\mathrm{C}$ & -5.41696100 & 2.26011300 & -1.72012600 \\
\hline $\mathrm{C}$ & -5.70914200 & 0.75199600 & 0.17067400 \\
\hline $\mathrm{C}$ & -6.72899500 & 1.98639600 & -2.13650700 \\
\hline $\mathrm{H}$ & -4.78335200 & 2.94582900 & -2.30479100 \\
\hline $\mathrm{C}$ & -7.02765900 & 0.49339200 & -0.24295000 \\
\hline $\mathrm{H}$ & -5.30786500 & 0.26008500 & 1.07173300 \\
\hline $\mathrm{C}$ & -7.54309800 & 1.10421800 & -1.40033100 \\
\hline $\mathrm{H}$ & -7.12086200 & 2.46760800 & -3.04722200 \\
\hline $\mathrm{H}$ & -7.65486800 & -0.19797900 & 0.34297700 \\
\hline $\mathrm{C}$ & 5.69061000 & 1.92552400 & -0.95151900 \\
\hline $\mathrm{C}$ & 6.91795800 & 2.09799600 & -1.63619100 \\
\hline $\mathrm{C}$ & 5.16830100 & 3.01120900 & -0.21373300 \\
\hline $\mathrm{C}$ & 7.61519600 & 3.31488600 & -1.56520000 \\
\hline $\mathrm{H}$ & 7.33159700 & 1.26786500 & -2.23360800 \\
\hline $\mathrm{C}$ & 5.86163000 & 4.23469300 & -0.15425300 \\
\hline $\mathrm{H}$ & 4.21085800 & 2.90049900 & 0.31892000 \\
\hline $\mathrm{C}$ & 7.08708300 & 4.38954500 & -0.82500600 \\
\hline $\mathrm{H}$ & 8.57206200 & 3.42890400 & -2.09980900 \\
\hline $\mathrm{H}$ & 5.43953400 & 5.07239100 & 0.42445400 \\
\hline $\mathrm{H}$ & 7.62850100 & 5.34783900 & -0.77667800 \\
\hline $\mathrm{C}$ & 5.78454800 & -0.77916500 & 0.03468500 \\
\hline $\mathrm{C}$ & 6.41953400 & -0.27947400 & 1.19488200 \\
\hline $\mathrm{C}$ & 5.89918900 & -2.15674700 & -0.26180900 \\
\hline $\mathrm{C}$ & 7.13498000 & -1.14081800 & 2.04525800 \\
\hline $\mathrm{H}$ & 6.35931800 & 0.79500800 & 1.43083000 \\
\hline $\mathrm{C}$ & 6.61042200 & -3.01966100 & 0.59150100 \\
\hline $\mathrm{H}$ & 5.43398300 & -2.55534800 & -1.17845100 \\
\hline $\mathrm{C}$ & 7.22931000 & -2.51298800 & 1.74807500 \\
\hline $\mathrm{H}$ & 7.62549300 & -0.73638500 & 2.94551700 \\
\hline $\mathrm{H}$ & 6.69087200 & -4.09063200 & 0.34480800 \\
\hline $\mathrm{H}$ & 7.79359400 & -3.18563600 & 2.41367700 \\
\hline $\mathrm{C}$ & -1.98960500 & -1.56851700 & 1.80268800 \\
\hline $\mathrm{C}$ & -2.33253700 & -2.79999200 & 2.40321900 \\
\hline $\mathrm{C}$ & -1.80972000 & -0.43606400 & 2.62889600 \\
\hline
\end{tabular}




$\begin{array}{lrrr}\mathrm{C} & -2.48487100 & -2.89457300 & 3.79874700 \\ \mathrm{H} & -2.48765000 & -3.69203900 & 1.77631200 \\ \mathrm{C} & -1.94929900 & -0.53257600 & 4.02431800 \\ \mathrm{H} & -1.56109400 & 0.53234300 & 2.16370300 \\ \mathrm{C} & -2.29033000 & -1.76433600 & 4.61273300 \\ \mathrm{H} & -2.75836700 & -3.86076100 & 4.25268800 \\ \mathrm{H} & -1.80019000 & 0.35903700 & 4.65447600 \\ \mathrm{H} & -2.41055500 & -1.84140000 & 5.70510400 \\ \mathrm{C} & -2.71736500 & -2.79658100 & -0.72620000 \\ \mathrm{C} & -2.11372000 & -4.04264600 & -1.00664300 \\ \mathrm{C} & -4.09271600 & -2.62176200 & -1.00488300 \\ \mathrm{C} & -2.87489600 & -5.09582600 & -1.54588000 \\ \mathrm{H} & -1.04086700 & -4.19068200 & -0.80748000 \\ \mathrm{C} & -4.85193000 & -3.67825100 & -1.53509800 \\ \mathrm{H} & -4.57098800 & -1.64698000 & -0.81152500 \\ \mathrm{C} & -4.24467900 & -4.91779800 & -1.80847000 \\ \mathrm{H} & -2.39145900 & -6.06187600 & -1.76364500 \\ \mathrm{H} & -5.92239800 & -3.52701400 & -1.74728700 \\ \mathrm{H} & -4.83813100 & -5.74298700 & -2.23361400 \\ \mathrm{C} & -3.21937600 & 3.11388400 & 0.58363600 \\ \mathrm{C} & -4.17487600 & 4.24012500 & 0.88594200 \\ \mathrm{H} & -2.16862200 & 3.27883200 & 0.89401200 \\ \mathrm{H} & -3.28713500 & 1.83109000 & 1.09807200 \\ \mathrm{C} & -4.02303100 & 4.83320200 & 2.30002100 \\ \mathrm{H} & -3.97895000 & 5.04295300 & 0.13346800 \\ \mathrm{H} & -4.22485400 & 4.03544500 & 3.04983100 \\ \mathrm{H} & -2.96349500 & 5.13723200 & 2.45808600 \\ \mathrm{H} & -8.57221600 & 0.89157100 & -1.73019400 \\ \mathrm{H} & -5.22044900 & 3.91129500 & 0.70534100 \\ \mathrm{C} & -4.94784700 & 6.02947200 & 2.55372500 \\ \mathrm{H} & -4.82007300 & 6.43008400 & 3.58044800 \\ \mathrm{H} & -4.74207600 & 6.85779300 & 1.84244600 \\ \mathrm{H} & -6.01579800 & 5.74819700 & 2.43431300\end{array}$

\section{TS7-Trans Hydrogen Migration TS with one Phosphine Dissociated}

Electronic Contribution to Gibbs free energy in toluene at 373.15 K (BLYP/6-31g*): -5307.08456596

Energies and corrections (BP86/tzvp-svp):

$\begin{array}{lc}\text { Zero-point correction= } & 0.716176 \text { (Hartree/Particle) } \\ \text { Thermal correction to Energy= } & 0.766516 \\ \text { Thermal correction to Enthalpy= } & 0.767461 \\ \text { Thermal correction to Gibbs Free Energy= } & 0.619996 \\ \text { Sum of electronic and zero-point Energies }= & -5306.701329 \\ \text { Sum of electronic and thermal Energies }= & -5306.650988 \\ \text { Sum of electronic and thermal Enthalpies }= & -5306.650044 \\ \text { Sum of electronic and thermal Free Energies }= & -5306.797509\end{array}$

Coordinates:

$\begin{array}{lrrr}\mathrm{C} & 0.31201500 & -1.43827600 & -1.71208900 \\ \mathrm{C} & 1.58122300 & -2.11342800 & -1.69565400 \\ \mathrm{C} & 1.72476000 & -2.77347400 & -0.42265800 \\ \mathrm{C} & 0.53718800 & -2.51754800 & 0.34871900 \\ \mathrm{C} & -0.35030600 & -1.69048800 & -0.44626400 \\ \mathrm{H} & -0.08980100 & -0.83248300 & -2.53413300 \\ \mathrm{H} & 2.32507100 & -2.09274500 & -2.50324200 \\ \mathrm{H} & 2.59758000 & -3.34995900 & -0.08858400 \\ \mathrm{H} & 0.33655100 & -2.87151200 & 1.36790200 \\ \mathrm{Fe} & 1.44878800 & -0.75180100 & -0.15881800 \\ \mathrm{C} & 2.02646000 & 1.14586100 & -0.64076000\end{array}$




\begin{tabular}{|c|c|c|c|}
\hline $\mathrm{C}$ & 1.04283300 & 1.17573200 & 0.40773800 \\
\hline $\mathrm{C}$ & 3.16973200 & 0.38273300 & -0.17249900 \\
\hline $\mathrm{H}$ & 1.92824700 & 1.61102600 & -1.63064900 \\
\hline $\mathrm{C}$ & 1.55422100 & 0.41824000 & 1.52315100 \\
\hline $\mathrm{H}$ & 0.04453600 & 1.63366000 & 0.33307400 \\
\hline $\mathrm{C}$ & 2.86039700 & -0.07136700 & 1.16866000 \\
\hline $\mathrm{H}$ & 1.03247500 & 0.22457400 & 2.46960600 \\
\hline $\mathrm{H}$ & 3.51030200 & -0.69535400 & 1.79539300 \\
\hline $\mathrm{P}$ & -2.04777700 & -1.10018400 & -0.04157800 \\
\hline $\mathrm{P}$ & 4.67130500 & 0.10581300 & -1.20349700 \\
\hline $\mathrm{Ni}$ & -2.56482600 & 1.00049100 & -0.57800600 \\
\hline $\mathrm{O}$ & -1.55860500 & 1.84272000 & -3.18807700 \\
\hline $\mathrm{C}$ & -1.96833400 & 1.49087200 & -2.14864000 \\
\hline $\mathrm{C}$ & -3.65551900 & 2.20916800 & 0.40804800 \\
\hline $\mathrm{C}$ & -5.08875200 & 1.99503100 & 0.04743100 \\
\hline $\mathrm{C}$ & -5.69626900 & 2.84776100 & -0.90887200 \\
\hline $\mathrm{C}$ & -5.86647800 & 0.95882400 & 0.62054300 \\
\hline $\mathrm{C}$ & -7.03532200 & 2.65980700 & -1.28615300 \\
\hline $\mathrm{H}$ & -5.09944500 & 3.65265500 & -1.36719500 \\
\hline $\mathrm{C}$ & -7.21304500 & 0.78808000 & 0.25493400 \\
\hline $\mathrm{H}$ & -5.40728700 & 0.29062800 & 1.36696100 \\
\hline $\mathrm{C}$ & -7.80162800 & 1.63175800 & -0.70478100 \\
\hline $\mathrm{H}$ & -7.48670800 & 3.32463400 & -2.04034700 \\
\hline $\mathrm{H}$ & -7.80563900 & -0.01526900 & 0.72185500 \\
\hline $\mathrm{C}$ & 5.70982100 & 1.58975700 & -0.75967100 \\
\hline $\mathrm{C}$ & 6.92198700 & 1.76431100 & -1.47028300 \\
\hline $\mathrm{C}$ & 5.33141300 & 2.56960900 & 0.18515600 \\
\hline $\mathrm{C}$ & 7.74468600 & 2.87568800 & -1.22506700 \\
\hline $\mathrm{H}$ & 7.22376900 & 1.01999400 & -2.22661000 \\
\hline $\mathrm{C}$ & 6.15049600 & 3.68979700 & 0.42052100 \\
\hline $\mathrm{H}$ & 4.38788500 & 2.45726200 & 0.74183700 \\
\hline $\mathrm{C}$ & 7.35962300 & 3.84462900 & -0.27929300 \\
\hline $\mathrm{H}$ & 8.68750900 & 2.99206400 & -1.78357400 \\
\hline $\mathrm{H}$ & 5.84006100 & 4.44597300 & 1.15985800 \\
\hline $\mathrm{H}$ & 7.99953600 & 4.72196000 & -0.09328400 \\
\hline $\mathrm{C}$ & 5.54291000 & -1.24077600 & -0.24882700 \\
\hline $\mathrm{C}$ & 6.24698600 & -1.01645200 & 0.95648300 \\
\hline $\mathrm{C}$ & 5.51364900 & -2.54870000 & -0.78468900 \\
\hline $\mathrm{C}$ & 6.88857500 & -2.07858800 & 1.61762500 \\
\hline $\mathrm{H}$ & 6.29956800 & 0.00036000 & 1.37737200 \\
\hline $\mathrm{C}$ & 6.15124700 & -3.61247100 & -0.12092000 \\
\hline $\mathrm{H}$ & 4.99366200 & -2.73260800 & -1.73934600 \\
\hline $\mathrm{C}$ & 6.83930100 & -3.37932500 & 1.08307900 \\
\hline $\mathrm{H}$ & 7.43427300 & -1.88811300 & 2.55600300 \\
\hline $\mathrm{H}$ & 6.11970300 & -4.62569800 & -0.55298700 \\
\hline $\mathrm{H}$ & 7.34594100 & -4.20958400 & 1.60063500 \\
\hline $\mathrm{C}$ & -2.20145400 & -1.63293600 & 1.74262100 \\
\hline $\mathrm{C}$ & -2.59499600 & -2.93062300 & 2.13761100 \\
\hline $\mathrm{C}$ & -1.91970500 & -0.67429000 & 2.74192200 \\
\hline $\mathrm{C}$ & -2.69585400 & -3.26064500 & 3.50168000 \\
\hline $\mathrm{H}$ & -2.83149300 & -3.68931200 & 1.37526800 \\
\hline $\mathrm{C}$ & -2.00696900 & -1.00729000 & 4.10470500 \\
\hline $\mathrm{H}$ & -1.63693600 & 0.34791400 & 2.43939600 \\
\hline $\mathrm{C}$ & -2.39833500 & -2.30333400 & 4.48789600 \\
\hline $\mathrm{H}$ & -3.01065900 & -4.27523200 & 3.79475500 \\
\hline $\mathrm{H}$ & -1.77866300 & -0.24905400 & 4.87103800 \\
\hline $\mathrm{H}$ & -2.47851400 & -2.56445600 & 5.55516800 \\
\hline $\mathrm{C}$ & -3.10060900 & -2.35158200 & -0.93326000 \\
\hline $\mathrm{C}$ & -2.59799400 & -3.59314900 & -1.38410700 \\
\hline $\mathrm{C}$ & -4.45136100 & -2.02490100 & -1.19244100 \\
\hline $\mathrm{C}$ & -3.43472700 & -4.49357700 & -2.06802300 \\
\hline $\mathrm{H}$ & -1.54400900 & -3.85725500 & -1.20570 \\
\hline
\end{tabular}




$\begin{array}{lrrr}\mathrm{C} & -5.28666800 & -2.92945200 & -1.86972300 \\ \mathrm{H} & -4.85155200 & -1.05084400 & -0.86639100 \\ \mathrm{C} & -4.78061300 & -4.16591600 & -2.31002400 \\ \mathrm{H} & -3.02906600 & -5.45706300 & -2.41658900 \\ \mathrm{H} & -6.33688200 & -2.65925800 & -2.06414100 \\ \mathrm{H} & -5.43328000 & -4.87110100 & -2.84913600 \\ \mathrm{C} & -3.41058900 & 3.33659400 & 1.24863100 \\ \mathrm{C} & -2.03916400 & 3.81666500 & 1.64321700 \\ \mathrm{H} & -4.26787400 & 3.98290200 & 1.53614100 \\ \mathrm{H} & -3.43848700 & 1.99649600 & 1.62921400 \\ \mathrm{C} & -1.56619500 & 4.97925800 & 0.73657200 \\ \mathrm{H} & -2.03738900 & 4.16055100 & 2.70216700 \\ \mathrm{H} & -1.55930100 & 4.62492500 & -0.31730000 \\ \mathrm{H} & -2.31193300 & 5.80431700 & 0.78071900 \\ \mathrm{H} & -8.85307700 & 1.48837400 & -1.00006600 \\ \mathrm{H} & -1.31466400 & 2.97626600 & 1.55845600 \\ \mathrm{C} & -0.18091700 & 5.50988000 & 1.12473300 \\ \mathrm{H} & 0.13126100 & 6.34015300 & 0.45825300 \\ \mathrm{H} & -0.16990600 & 5.89369700 & 2.16736100 \\ \mathrm{H} & 0.59088100 & 4.71457500 & 1.05316000\end{array}$

p-Fluorophenyl isobutyl ketene

Coordinates:

$\begin{array}{lrrr}\mathrm{O} & -1.57496000 & 2.70697100 & -0.50072400 \\ \mathrm{C} & -1.17055900 & 1.60867100 & -0.37358800 \\ \mathrm{C} & -0.71938900 & 0.37096800 & -0.21537900 \\ \mathrm{C} & 0.74000500 & 0.13685800 & -0.10481200 \\ \mathrm{C} & 1.26357100 & -1.17765200 & -0.19544900 \\ \mathrm{C} & 1.65954300 & 1.20183900 & 0.09414000 \\ \mathrm{C} & 2.64394500 & -1.42129300 & -0.09213300 \\ \mathrm{H} & 0.59142900 & -2.03287200 & -0.35563900 \\ \mathrm{C} & 3.03709300 & 0.97113500 & 0.19100100 \\ \mathrm{H} & 1.29075500 & 2.23690100 & 0.18442900 \\ \mathrm{C} & 3.51727500 & -0.34463000 & 0.09782700 \\ \mathrm{H} & 3.04769300 & -2.44266500 & -0.16185300 \\ \mathrm{H} & 3.74515400 & 1.79889900 & 0.34694700 \\ \mathrm{C} & -1.71718900 & -0.78739200 & -0.24305700 \\ \mathrm{H} & -1.57982900 & -1.34245400 & -1.19951900 \\ \mathrm{H} & -1.43051400 & -1.49372200 & 0.56958000 \\ \mathrm{C} & -3.21119100 & -0.42645200 & -0.07739000 \\ \mathrm{H} & -3.45945000 & 0.36589400 & -0.82325400 \\ \mathrm{C} & -4.08379900 & -1.65119500 & -0.40402200 \\ \mathrm{H} & -3.86764900 & -2.49001400 & 0.29330900 \\ \mathrm{H} & -3.90727700 & -2.01770500 & -1.43696200 \\ \mathrm{H} & -5.16285300 & -1.40996400 & -0.31000400 \\ \mathrm{C} & -3.53158100 & 0.11549700 & 1.32793000 \\ \mathrm{H} & -3.32568600 & -0.65610100 & 2.10129100 \\ \mathrm{H} & -2.93160600 & 1.01237700 & 1.58526600 \\ \mathrm{H} & -4.60187700 & 0.39684700 & 1.40996900 \\ \mathrm{~F} & 4.84722800 & -0.57325700 & 0.19686800\end{array}$

\section{Carbonyl Carbene Complex of 8d}

Coordinates:

$\begin{array}{lrrr}C & -0.63105100 & -3.06644200 & 0.84706500 \\ \mathrm{C} & -1.20944500 & -4.28423300 & 0.34606500 \\ \mathrm{C} & -0.56745000 & -4.60448700 & -0.90109400 \\ \mathrm{C} & 0.41514200 & -3.58975300 & -1.17415000 \\ \mathrm{C} & 0.38564800 & -2.62533300 & -0.09030600\end{array}$




\begin{tabular}{|c|c|c|c|}
\hline $\mathrm{H}$ & -0.91995300 & -2.54919600 & 1.77000700 \\
\hline $\mathrm{H}$ & -2.01947500 & -4.85254800 & 0.82213700 \\
\hline $\mathrm{H}$ & -0.80192200 & -5.46153500 & -1.54670500 \\
\hline $\mathrm{H}$ & 1.06237900 & -3.53829400 & -2.05819800 \\
\hline $\mathrm{Fe}$ & -1.43941400 & -2.73817800 & -0.99804800 \\
\hline $\mathrm{C}$ & -1.66968100 & -1.15307600 & -2.26081000 \\
\hline $\mathrm{C}$ & -2.20961200 & -2.34020600 & -2.86814600 \\
\hline $\mathrm{C}$ & -2.36778300 & -0.91866800 & -1.00935400 \\
\hline $\mathrm{H}$ & -0.85445700 & -0.53950900 & -2.66253500 \\
\hline $\mathrm{C}$ & -3.23715000 & -2.85453400 & -2.00263100 \\
\hline $\mathrm{H}$ & -1.87503200 & -2.78715300 & -3.81372400 \\
\hline $\mathrm{C}$ & -3.33974300 & -1.98508600 & -0.86105500 \\
\hline $\mathrm{H}$ & -3.82489000 & -3.76724200 & -2.16976000 \\
\hline $\mathrm{H}$ & -4.01636200 & -2.11892000 & -0.00825500 \\
\hline $\mathrm{P}$ & 1.35088400 & -1.06266100 & 0.07915000 \\
\hline $\mathrm{P}$ & -1.96022700 & 0.45435400 & 0.15761900 \\
\hline $\mathrm{Ni}$ & 0.29231000 & 0.85985200 & 0.58899200 \\
\hline $\mathrm{O}$ & 0.81473800 & 1.17151900 & 3.45975500 \\
\hline $\mathrm{C}$ & 0.55520100 & 0.96536900 & 2.33894600 \\
\hline $\mathrm{C}$ & 0.92921700 & 2.40057100 & -0.22842000 \\
\hline $\mathrm{C}$ & 2.31206200 & 2.82086600 & 0.10490400 \\
\hline $\mathrm{C}$ & 2.60382000 & 3.45176500 & 1.34685900 \\
\hline $\mathrm{C}$ & 3.40009200 & 2.58659900 & -0.78137000 \\
\hline $\mathrm{C}$ & 3.91286000 & 3.83670700 & 1.67635100 \\
\hline $\mathrm{H}$ & 1.78608100 & 3.63335400 & 2.06019200 \\
\hline $\mathrm{C}$ & 4.71023600 & 2.95932300 & -0.43605300 \\
\hline $\mathrm{H}$ & 3.21904400 & 2.07703500 & -1.73883300 \\
\hline $\mathrm{C}$ & 4.97876700 & 3.59261000 & 0.79041400 \\
\hline $\mathrm{H}$ & 4.10096600 & 4.32748600 & 2.64537800 \\
\hline $\mathrm{H}$ & 5.53163800 & 2.74827700 & -1.14039300 \\
\hline $\mathrm{C}$ & -3.00815400 & 1.84135800 & -0.53021400 \\
\hline $\mathrm{C}$ & -3.24212100 & 2.97155300 & 0.28971300 \\
\hline $\mathrm{C}$ & -3.53164300 & 1.83606800 & -1.84198600 \\
\hline $\mathrm{C}$ & -3.98577700 & 4.06210600 & -0.18814300 \\
\hline $\mathrm{H}$ & -2.84266800 & 2.99428600 & 1.31701800 \\
\hline $\mathrm{C}$ & -4.26867700 & 2.93502700 & -2.32250800 \\
\hline $\mathrm{H}$ & -3.37053800 & 0.96455500 & -2.49494600 \\
\hline $\mathrm{C}$ & -4.49986800 & 4.05000100 & -1.49899700 \\
\hline $\mathrm{H}$ & -4.16427100 & 4.92962300 & 0.46746100 \\
\hline $\mathrm{H}$ & -4.67165900 & 2.91249200 & -3.34806100 \\
\hline $\mathrm{H}$ & -5.08091300 & 4.90717600 & -1.87478900 \\
\hline $\mathrm{C}$ & -3.03588500 & -0.01691000 & 1.62139800 \\
\hline $\mathrm{C}$ & -4.44727200 & 0.06369100 & 1.55496000 \\
\hline $\mathrm{C}$ & -2.44262100 & -0.47398800 & 2.81595300 \\
\hline $\mathrm{C}$ & -5.23873600 & -0.31407500 & 2.65304900 \\
\hline $\mathrm{H}$ & -4.93387900 & 0.43160200 & 0.63807600 \\
\hline $\mathrm{C}$ & -3.23364400 & -0.85292800 & 3.91639200 \\
\hline $\mathrm{H}$ & -1.34499500 & -0.51759800 & 2.88691500 \\
\hline $\mathrm{C}$ & -4.63427100 & -0.77503700 & 3.83747600 \\
\hline $\mathrm{H}$ & -6.33631600 & -0.24323000 & 2.58400600 \\
\hline $\mathrm{H}$ & -2.74839600 & -1.20200700 & 4.84187400 \\
\hline $\mathrm{H}$ & -5.25576000 & -1.06555900 & 4.69959600 \\
\hline $\mathrm{C}$ & 2.30979400 & -1.08951600 & -1.52623100 \\
\hline $\mathrm{C}$ & 3.38534200 & -1.98272300 & -1.73200300 \\
\hline $\mathrm{C}$ & 1.93738800 & -0.22030300 & -2.57133600 \\
\hline $\mathrm{C}$ & 4.06346500 & -2.00854900 & -2.96355100 \\
\hline $\mathrm{H}$ & 3.70196000 & -2.65873300 & -0.92221500 \\
\hline $\mathrm{C}$ & 2.60793300 & -0.25219000 & -3.80728200 \\
\hline $\mathrm{H}$ & 1.12637500 & 0.50492700 & -2.39204000 \\
\hline $\mathrm{C}$ & 3.67391200 & -1.14752000 & -4.00578800 \\
\hline $\mathrm{H}$ & 4.90552200 & -2.70452300 & -3.10764200 \\
\hline $\mathrm{H}$ & 2.30339900 & 0.43351500 & -4.6143470 \\
\hline
\end{tabular}




$\begin{array}{lrrr}\mathrm{H} & 4.20741000 & -1.16873700 & -4.96948300 \\ \mathrm{C} & 2.67592000 & -1.53949900 & 1.29624800 \\ \mathrm{C} & 2.77754000 & -2.83222300 & 1.85503500 \\ \mathrm{C} & 3.60827200 & -0.54781900 & 1.68176400 \\ \mathrm{C} & 3.79333200 & -3.12764100 & 2.78361200 \\ \mathrm{H} & 2.05938300 & -3.61513900 & 1.56662100 \\ \mathrm{C} & 4.62723600 & -0.85074000 & 2.59919600 \\ \mathrm{H} & 3.53510900 & 0.46884700 & 1.26261100 \\ \mathrm{C} & 4.72114900 & -2.14014100 & 3.15594700 \\ \mathrm{H} & 3.85795400 & -4.13915600 & 3.21620900 \\ \mathrm{H} & 5.34747600 & -0.06869200 & 2.88736300 \\ \mathrm{H} & 5.51570000 & -2.37277400 & 3.88287600 \\ \mathrm{C} & 0.15618700 & 3.39307000 & -1.08727500 \\ \mathrm{C} & 0.83278100 & 3.71014600 & -2.44202500 \\ \mathrm{C} & -0.08071600 & 4.70639700 & -0.29849600 \\ \mathrm{H} & -0.83825000 & 2.95550100 & -1.30517000 \\ \mathrm{H} & 1.00720100 & 2.79487600 & -3.04409200 \\ \mathrm{H} & 0.17180800 & 4.37823300 & -3.03340400 \\ \mathrm{H} & 1.80674500 & 4.22388800 & -2.30956200 \\ \mathrm{H} & -0.57507500 & 4.51280800 & 0.67468600 \\ \mathrm{H} & 0.87282900 & 5.23916800 & -0.10273100 \\ \mathrm{H} & -0.74024600 & 5.37800900 & -0.88713300 \\ \mathrm{H} & 6.00644600 & 3.88648400 & 1.05614800\end{array}$


Table S4a crystal data and structure refinement for $\mathbf{8 a}$

$\begin{array}{ll}\text { Table S4d anisotropic displacement paramaters for 8a } & \text { S75 }\end{array}$

$\begin{array}{ll}\text { Table S4e hydrogen coordinates for 8a } & \text { S77 }\end{array}$

$\begin{array}{lr}\text { Table S4f torsions angles for 8a } & \text { S79 }\end{array}$

$\begin{array}{ll}\text { Table S4g least squares planes for 8a } & \text { S89 }\end{array}$

$\begin{array}{ll}\text { Table S5a crystal data and structure refinement for } \mathbf{8 b} & \text { S90 }\end{array}$

Table S5b coordinates for $\mathbf{8 b} \quad$ S91

$\begin{array}{ll}\text { Table S5c bond lengths and angles } 8 \mathbf{b} & \text { S92 }\end{array}$

Table S5d anisotropic displacement parameters for $\mathbf{8 b} \quad$ S99

$\begin{array}{ll}\text { Table S5e hydrogen coordinates for } \mathbf{8 b} & \text { S100 }\end{array}$

Table S5f torsion angles for $\mathbf{8 b} \quad$ S101

Table S6a sample and crystal data for 8e $\quad$ S106

Table S6b data collection and structure refinement for $\mathbf{8 e} \quad$ S106

$\begin{array}{ll}\text { Table S6c coordinates for } 8 \mathbf{e} & \text { S107 }\end{array}$

$\begin{array}{ll}\text { Table S6d bond lengths for } 8 \mathbf{e} & \text { S109 }\end{array}$

Table S6e bond angles for $8 \mathbf{e} \quad$ S111

Table S6f torsion angles for 8e $\quad$ S116

Table S6g anisotropic displacement parameters for $8 \mathbf{e} \quad$ S118

$\begin{array}{ll}\text { Table S6h hydrogen coordinates for } 8 \mathbf{e} & \text { S120 }\end{array}$

$\begin{array}{ll}\text { Table S7a sample and crystal data for 8ca } & \text { S123 }\end{array}$

Table S7b data collection and structure refinement for 8ca $\quad$ S123

$\begin{array}{ll}\text { Table S7c coordinates for 8ca } & \text { S124 }\end{array}$

Table S7d bond lengths for 8ca $\quad$ S126

$\begin{array}{ll}\text { Table S7e bond angles for 8ca } & \text { S128 }\end{array}$

$\begin{array}{ll}\text { Table S7f torsion angles for 8ca } & \text { S132 }\end{array}$

Table S7g anisotropic displacement parameters for 8ca $\quad$ S135

$\begin{array}{ll}\text { Table S7h hydrogen coordinates for 8ca } & \text { S137 }\end{array}$

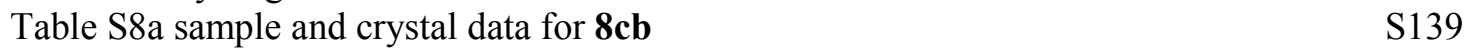

$\begin{array}{ll}\text { Table S8b data collection and structure refinement for } \mathbf{8 c b} & \text { S139 }\end{array}$

$\begin{array}{lr}\text { Table S8c coordinates for } \mathbf{8 c b} & \text { S140 }\end{array}$

$\begin{array}{ll}\text { Table S8d bond lengths for } \mathbf{8 c b} & \text { S142 }\end{array}$

$\begin{array}{ll}\text { Table S8e bond angles for } \mathbf{8 c b} & \text { S144 }\end{array}$

$\begin{array}{ll}\text { Table S8f torsion angles for } \mathbf{8 c b} & \text { S148 }\end{array}$

Table S8g anisotropic displacement parameters for $\mathbf{8 c b} \quad \mathrm{S} 151$

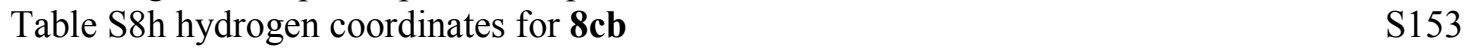

Table S9a sample and crystal data for 8cc $\quad$ S155

Table S9b data collection and structure refinement for 8cc $\quad$ S155

$\begin{array}{ll}\text { Table S9c coordinates for 8cc } & \text { S156 }\end{array}$

$\begin{array}{ll}\text { Table S9d bond lengths for 8cc } & \text { S158 }\end{array}$

$\begin{array}{ll}\text { Table S9e bond angles for 8cc } & \text { S161 }\end{array}$

Table S9f torsions angles for 8cc $\quad$ S165

Table S9g anisotropic displacement parameters for 8cc $\quad$ S168

$\begin{array}{ll}\text { Table S9h hydrogen coordinates for 8cc } & \text { S170 }\end{array}$ 
Table S4a. Crystal data and structure refinement for $\mathbf{8 a}$.

Identification code

Empirical formula

Formula weight

Temperature

Wavelength

Crystal system

Space group

Unit cell dimensions

Volume

Z

Density (calculated)

Absorption coefficient

$\mathrm{F}(000)$

Crystal size

Theta range for data collection

Index ranges

Reflections collected

Independent reflections

Completeness to theta $=27.48^{\circ}$

Absorption correction

Max. and min. transmission

Refinement method

Data / restraints / parameters

Goodness-of-fit on $\mathrm{F}^{2}$

Final R indices [I $>2 \operatorname{sigma}(\mathrm{I})]$

$\mathrm{R}$ indices (all data)

Extinction coefficient

Largest diff. peak and hole 8a

$\mathrm{C} 48 \mathrm{H} 38 \mathrm{Fe}$ Ni O P2

807.28

150(1) K

$0.71073 \AA$

Monoclinic

$\boldsymbol{P} 2{ }_{1} / n$

$\mathrm{a}=10.94830(10) \AA$

$\alpha=90^{\circ}$.

$\mathrm{b}=19.3974(2) \AA$

$\beta=106.7057(7)^{\circ}$.

$\mathrm{c}=18.5790(3) \AA$

$\gamma=90^{\circ}$.

3779.07(8) $\AA^{3}$

4

$1.419 \mathrm{Mg} / \mathrm{m}^{3}$

$1.004 \mathrm{~mm}^{-1}$

1672

$0.23 \times 0.15 \times 0.10 \mathrm{~mm}^{3}$

1.95 to $27.48^{\circ}$.

$-14<=\mathrm{h}<=14,-24<=\mathrm{k}<=25,-24<=1<=24$

16835

$8699[\mathrm{R}(\mathrm{int})=0.0295]$

$100.0 \%$

Multi-scan

0.9062 and 0.8019

Full-matrix least-squares on $\mathrm{F}^{2}$

8664 / 0 / 479

1.051

$\mathrm{R} 1=0.0347, \mathrm{wR} 2=0.0775$

$\mathrm{R} 1=0.0562, \mathrm{wR} 2=0.0847$

$0.00216(18)$

0.451 and -0.335 e. $\AA^{-3}$ 
Table S4b. Atomic coordinates (x $\left.10^{4}\right)$ and equivalent isotropic displacement parameters $\left(\AA^{2} \times 10^{3}\right)$ for 8a. $U(e q)$ is defined as one third of the trace of the orthogonalized $U^{i j}$ tensor.

\begin{tabular}{|c|c|c|c|c|}
\hline & $\mathrm{x}$ & $\mathrm{y}$ & $\mathrm{z}$ & $\mathrm{U}(\mathrm{eq})$ \\
\hline $\mathrm{Ni}(1)$ & $4698(1)$ & $1860(1)$ & $769(1)$ & $17(1)$ \\
\hline $\mathrm{Fe}(1)$ & $2200(1)$ & $2105(1)$ & $-1309(1)$ & $21(1)$ \\
\hline $\mathrm{P}(1)$ & $2756(1)$ & $1498(1)$ & $495(1)$ & $18(1)$ \\
\hline $\mathrm{P}(2)$ & $4664(1)$ & $2758(1)$ & $18(1)$ & $19(1)$ \\
\hline $\mathrm{O}(1)$ & $6434(1)$ & $1729(1)$ & $1234(1)$ & $21(1)$ \\
\hline $\mathrm{C}(1)$ & $5774(2)$ & 1281(1) & $1484(1)$ & $20(1)$ \\
\hline$C(2)$ & $6057(2)$ & $737(1)$ & $1957(1)$ & $20(1)$ \\
\hline$C(3)$ & $5017(2)$ & $349(1)$ & $2150(1)$ & $23(1)$ \\
\hline$C(4)$ & $4800(2)$ & $-339(1)$ & 1934(1) & $28(1)$ \\
\hline$C(5)$ & $3864(2)$ & $-718(1)$ & 2112(1) & $35(1)$ \\
\hline$C(6)$ & $3107(2)$ & $-419(1)$ & $2503(2)$ & $42(1)$ \\
\hline$C(7)$ & $3308(2)$ & $259(2)$ & $2725(2)$ & $43(1)$ \\
\hline$C(8)$ & $4273(2)$ & $637(1)$ & $2557(1)$ & $32(1)$ \\
\hline$C(9)$ & $7389(2)$ & $496(1)$ & $2287(1)$ & $22(1)$ \\
\hline$C(10)$ & $7694(2)$ & $34(1)$ & 2894(1) & $29(1)$ \\
\hline $\mathrm{C}(11)$ & $8934(2)$ & $-200(1)$ & $3200(1)$ & $36(1)$ \\
\hline$C(12)$ & $9904(2)$ & $20(1)$ & 2922(1) & $38(1)$ \\
\hline$C(13)$ & $9627(2)$ & $475(1)$ & $2329(2)$ & $37(1)$ \\
\hline$C(14)$ & $8386(2)$ & $708(1)$ & $2015(1)$ & $30(1)$ \\
\hline$C(15)$ & $2589(2)$ & $560(1)$ & $444(1)$ & $21(1)$ \\
\hline$C(16)$ & $3380(2)$ & $206(1)$ & $102(1)$ & $26(1)$ \\
\hline$C(17)$ & $3229(2)$ & $-499(1)$ & $-26(1)$ & $31(1)$ \\
\hline$C(18)$ & $2314(2)$ & $-858(1)$ & 201(1) & $33(1)$ \\
\hline$C(19)$ & $1538(2)$ & $-512(1)$ & $551(1)$ & $32(1)$ \\
\hline$C(20)$ & $1665(2)$ & 196(1) & $666(1)$ & $24(1)$ \\
\hline$C(21)$ & $1884(2)$ & $1806(1)$ & $1133(1)$ & $20(1)$ \\
\hline$C(22)$ & $556(2)$ & $1817(1)$ & $940(1)$ & $24(1)$ \\
\hline$C(23)$ & $-63(2)$ & $2085(1)$ & $1435(2)$ & $31(1)$ \\
\hline$C(24)$ & $633(2)$ & $2348(1)$ & $2121(2)$ & $35(1)$ \\
\hline$C(25)$ & 1951(2) & $2346(1)$ & $2318(1)$ & $33(1)$ \\
\hline$C(26)$ & $2573(2)$ & $2079(1)$ & $1824(1)$ & $25(1)$ \\
\hline
\end{tabular}




\begin{tabular}{|c|c|c|c|c|}
\hline$C(27)$ & $6240(2)$ & $3090(1)$ & $42(1)$ & $23(1)$ \\
\hline$C(28)$ & $7062(2)$ & $3288(1)$ & 733(1) & $30(1)$ \\
\hline$C(29)$ & $8274(2)$ & $3539(1)$ & $783(2)$ & $39(1)$ \\
\hline$C(30)$ & $8675(2)$ & $3577(1)$ & $152(2)$ & $44(1)$ \\
\hline $\mathrm{C}(31)$ & $7874(3)$ & $3381(2)$ & $-539(2)$ & $49(1)$ \\
\hline $\mathrm{C}(32)$ & $6649(2)$ & $3143(1)$ & $-592(2)$ & $37(1)$ \\
\hline$C(33)$ & $3812(2)$ & $3529(1)$ & $175(1)$ & $22(1)$ \\
\hline$C(34)$ & $3054(2)$ & $3480(1)$ & $655(1)$ & $27(1)$ \\
\hline$C(35)$ & $2311(2)$ & 4033(1) & 753(1) & $35(1)$ \\
\hline$C(36)$ & $2346(2)$ & $4645(1)$ & $377(2)$ & $37(1)$ \\
\hline$C(37)$ & $3130(2)$ & $4706(1)$ & $-82(2)$ & $36(1)$ \\
\hline $\mathrm{C}(38)$ & $3863(2)$ & $4153(1)$ & $-190(1)$ & $29(1)$ \\
\hline C(39) & $1665(2)$ & $1714(1)$ & $-423(1)$ & $21(1)$ \\
\hline$C(40)$ & $1256(2)$ & $1269(1)$ & $-1067(1)$ & $25(1)$ \\
\hline $\mathrm{C}(41)$ & $435(2)$ & $1650(1)$ & $-1665(1)$ & $31(1)$ \\
\hline$C(42)$ & $329(2)$ & $2327(1)$ & $-1410(1)$ & $31(1)$ \\
\hline$C(43)$ & $1080(2)$ & $2374(1)$ & $-649(1)$ & $25(1)$ \\
\hline $\mathrm{C}(44)$ & $3953(2)$ & $2545(1)$ & $-957(1)$ & $21(1)$ \\
\hline$C(45)$ & $4039(2)$ & 1864(1) & $-1237(1)$ & $22(1)$ \\
\hline$C(46)$ & $3287(2)$ & $1845(1)$ & $-2003(1)$ & $27(1)$ \\
\hline $\mathrm{C}(47)$ & $2744(2)$ & $2508(1)$ & $-2197(1)$ & $29(1)$ \\
\hline$C(48)$ & $3147(2)$ & 2942(1) & $-1560(1)$ & $26(1)$ \\
\hline
\end{tabular}


Table S4c. Bond lengths $[\AA]$ and angles $\left[{ }^{\circ}\right]$ for $\mathbf{8 a}$.

\begin{tabular}{|c|c|}
\hline $\mathrm{Ni}(1)-\mathrm{O}(1)$ & $1.8655(13)$ \\
\hline $\mathrm{Ni}(1)-\mathrm{C}(1)$ & $1.875(2)$ \\
\hline Ni(1)-P(1) & $2.1557(5)$ \\
\hline $\mathrm{Ni}(1)-\mathrm{P}(2)$ & $2.2252(6)$ \\
\hline $\mathrm{Fe}(1)-\mathrm{C}(44)$ & $2.029(2)$ \\
\hline $\mathrm{Fe}(1)-\mathrm{C}(45)$ & $2.033(2)$ \\
\hline $\mathrm{Fe}(1)-\mathrm{C}(43)$ & $2.037(2)$ \\
\hline $\mathrm{Fe}(1)-\mathrm{C}(40)$ & $2.042(2)$ \\
\hline $\mathrm{Fe}(1)-\mathrm{C}(39)$ & $2.046(2)$ \\
\hline $\mathrm{Fe}(1)-\mathrm{C}(42)$ & $2.048(2)$ \\
\hline $\mathrm{Fe}(1)-\mathrm{C}(48)$ & $2.050(2)$ \\
\hline $\mathrm{Fe}(1)-\mathrm{C}(46)$ & $2.052(2)$ \\
\hline $\mathrm{Fe}(1)-\mathrm{C}(41)$ & $2.054(2)$ \\
\hline $\mathrm{Fe}(1)-\mathrm{C}(47)$ & $2.062(2)$ \\
\hline $\mathrm{P}(1)-\mathrm{C}(21)$ & $1.824(2)$ \\
\hline $\mathrm{P}(1)-\mathrm{C}(39)$ & $1.827(2)$ \\
\hline $\mathrm{P}(1)-\mathrm{C}(15)$ & $1.829(2)$ \\
\hline $\mathrm{P}(2)-\mathrm{C}(44)$ & $1.802(2)$ \\
\hline $\mathrm{P}(2)-\mathrm{C}(33)$ & $1.829(2)$ \\
\hline $\mathrm{P}(2)-\mathrm{C}(27)$ & $1.830(2)$ \\
\hline $\mathrm{O}(1)-\mathrm{C}(1)$ & $1.297(2)$ \\
\hline $\mathrm{C}(1)-\mathrm{C}(2)$ & $1.351(3)$ \\
\hline $\mathrm{C}(2)-\mathrm{C}(9)$ & $1.485(3)$ \\
\hline $\mathrm{C}(2)-\mathrm{C}(3)$ & $1.492(3)$ \\
\hline $\mathrm{C}(3)-\mathrm{C}(8)$ & $1.379(3)$ \\
\hline$C(3)-C(4)$ & $1.395(3)$ \\
\hline$C(4)-C(5)$ & $1.377(3)$ \\
\hline $\mathrm{C}(4)-\mathrm{H}(4)$ & 0.9500 \\
\hline$C(5)-C(6)$ & $1.378(4)$ \\
\hline $\mathrm{C}(5)-\mathrm{H}(5)$ & 0.9500 \\
\hline$C(6)-C(7)$ & $1.377(4)$ \\
\hline $\mathrm{C}(6)-\mathrm{H}(6)$ & 0.9500 \\
\hline$C(7)-C(8)$ & $1.393(3)$ \\
\hline $\mathrm{C}(7)-\mathrm{H}(7)$ & 0.9500 \\
\hline
\end{tabular}




\begin{tabular}{|c|c|}
\hline $\mathrm{C}(8)-\mathrm{H}(8)$ & 0.9500 \\
\hline$C(9)-C(14)$ & $1.391(3)$ \\
\hline $\mathrm{C}(9)-\mathrm{C}(10)$ & $1.403(3)$ \\
\hline$C(10)-C(11)$ & $1.389(3)$ \\
\hline $\mathrm{C}(10)-\mathrm{H}(10)$ & 0.9500 \\
\hline$C(11)-C(12)$ & $1.375(4)$ \\
\hline $\mathrm{C}(11)-\mathrm{H}(11)$ & 0.9500 \\
\hline$C(12)-C(13)$ & $1.376(3)$ \\
\hline $\mathrm{C}(12)-\mathrm{H}(12)$ & 0.9500 \\
\hline$C(13)-C(14)$ & $1.391(3)$ \\
\hline $\mathrm{C}(13)-\mathrm{H}(13)$ & 0.9500 \\
\hline $\mathrm{C}(14)-\mathrm{H}(14)$ & 0.9500 \\
\hline$C(15)-C(20)$ & $1.390(3)$ \\
\hline$C(15)-C(16)$ & $1.394(3)$ \\
\hline$C(16)-C(17)$ & $1.389(3)$ \\
\hline $\mathrm{C}(16)-\mathrm{H}(16)$ & 0.9500 \\
\hline$C(17)-C(18)$ & $1.381(3)$ \\
\hline $\mathrm{C}(17)-\mathrm{H}(17)$ & 0.9500 \\
\hline$C(18)-C(19)$ & $1.384(3)$ \\
\hline $\mathrm{C}(18)-\mathrm{H}(18)$ & 0.9500 \\
\hline$C(19)-C(20)$ & $1.391(3)$ \\
\hline $\mathrm{C}(19)-\mathrm{H}(19)$ & 0.9500 \\
\hline $\mathrm{C}(20)-\mathrm{H}(20)$ & 0.9500 \\
\hline$C(21)-C(26)$ & $1.393(3)$ \\
\hline$C(21)-C(22)$ & $1.395(3)$ \\
\hline$C(22)-C(23)$ & $1.390(3)$ \\
\hline $\mathrm{C}(22)-\mathrm{H}(22)$ & 0.9500 \\
\hline$C(23)-C(24)$ & $1.381(4)$ \\
\hline $\mathrm{C}(23)-\mathrm{H}(23)$ & 0.9500 \\
\hline$C(24)-C(25)$ & $1.383(3)$ \\
\hline $\mathrm{C}(24)-\mathrm{H}(24)$ & 0.9500 \\
\hline$C(25)-C(26)$ & $1.391(3)$ \\
\hline $\mathrm{C}(25)-\mathrm{H}(25)$ & 0.9500 \\
\hline $\mathrm{C}(26)-\mathrm{H}(26)$ & 0.9500 \\
\hline$C(27)-C(32)$ & $1.378(3)$ \\
\hline $\mathrm{C}(27)-\mathrm{C}(28)$ & $1.394(3)$ \\
\hline
\end{tabular}




\begin{tabular}{|c|c|}
\hline$C(28)-C(29)$ & $1.392(3)$ \\
\hline $\mathrm{C}(28)-\mathrm{H}(28)$ & 0.9500 \\
\hline$C(29)-C(30)$ & $1.366(4)$ \\
\hline $\mathrm{C}(29)-\mathrm{H}(29)$ & 0.9500 \\
\hline$C(30)-C(31)$ & $1.384(4)$ \\
\hline $\mathrm{C}(30)-\mathrm{H}(30)$ & 0.9500 \\
\hline$C(31)-C(32)$ & $1.396(3)$ \\
\hline $\mathrm{C}(31)-\mathrm{H}(31)$ & 0.9500 \\
\hline $\mathrm{C}(32)-\mathrm{H}(32)$ & 0.9500 \\
\hline$C(33)-C(34)$ & $1.385(3)$ \\
\hline$C(33)-C(38)$ & $1.396(3)$ \\
\hline$C(34)-C(35)$ & $1.389(3)$ \\
\hline $\mathrm{C}(34)-\mathrm{H}(34)$ & 0.9500 \\
\hline$C(35)-C(36)$ & $1.382(4)$ \\
\hline $\mathrm{C}(35)-\mathrm{H}(35)$ & 0.9500 \\
\hline$C(36)-C(37)$ & $1.380(4)$ \\
\hline $\mathrm{C}(36)-\mathrm{H}(36)$ & 0.9500 \\
\hline $\mathrm{C}(37)-\mathrm{C}(38)$ & $1.388(3)$ \\
\hline $\mathrm{C}(37)-\mathrm{H}(37)$ & 0.9500 \\
\hline $\mathrm{C}(38)-\mathrm{H}(38)$ & 0.9500 \\
\hline $\mathrm{C}(39)-\mathrm{C}(40)$ & $1.439(3)$ \\
\hline C(39)-C(43) & $1.439(3)$ \\
\hline$C(40)-C(41)$ & $1.420(3)$ \\
\hline $\mathrm{C}(40)-\mathrm{H}(40)$ & 0.9500 \\
\hline $\mathrm{C}(41)-\mathrm{C}(42)$ & $1.414(3)$ \\
\hline $\mathrm{C}(41)-\mathrm{H}(41)$ & 0.9500 \\
\hline $\mathrm{C}(42)-\mathrm{C}(43)$ & $1.420(3)$ \\
\hline $\mathrm{C}(42)-\mathrm{H}(42)$ & 0.9500 \\
\hline $\mathrm{C}(43)-\mathrm{H}(43)$ & 0.9500 \\
\hline $\mathrm{C}(44)-\mathrm{C}(45)$ & $1.432(3)$ \\
\hline $\mathrm{C}(44)-\mathrm{C}(48)$ & $1.435(3)$ \\
\hline $\mathrm{C}(45)-\mathrm{C}(46)$ & $1.425(3)$ \\
\hline $\mathrm{C}(45)-\mathrm{H}(45)$ & 0.9500 \\
\hline $\mathrm{C}(46)-\mathrm{C}(47)$ & $1.420(3)$ \\
\hline $\mathrm{C}(46)-\mathrm{H}(46)$ & 0.9500 \\
\hline $\mathrm{C}(47)-\mathrm{C}(48)$ & $1.417(3)$ \\
\hline
\end{tabular}




\begin{tabular}{|c|c|}
\hline $\mathrm{C}(47)-\mathrm{H}(47)$ & 0.9500 \\
\hline $\mathrm{C}(48)-\mathrm{H}(48)$ & 0.9500 \\
\hline $\mathrm{O}(1)-\mathrm{Ni}(1)-\mathrm{C}(1)$ & $40.60(7)$ \\
\hline $\mathrm{O}(1)-\mathrm{Ni}(1)-\mathrm{P}(1)$ & $150.35(5)$ \\
\hline $\mathrm{C}(1)-\mathrm{Ni}(1)-\mathrm{P}(1)$ & $110.41(6)$ \\
\hline $\mathrm{O}(1)-\mathrm{Ni}(1)-\mathrm{P}(2)$ & $103.21(5)$ \\
\hline$C(1)-N i(1)-P(2)$ & $143.79(6)$ \\
\hline $\mathrm{P}(1)-\mathrm{Ni}(1)-\mathrm{P}(2)$ & $105.60(2)$ \\
\hline $\mathrm{C}(44)-\mathrm{Fe}(1)-\mathrm{C}(45)$ & $41.27(8)$ \\
\hline $\mathrm{C}(44)-\mathrm{Fe}(1)-\mathrm{C}(43)$ & $112.04(9)$ \\
\hline $\mathrm{C}(45)-\mathrm{Fe}(1)-\mathrm{C}(43)$ & $140.85(8)$ \\
\hline $\mathrm{C}(44)-\mathrm{Fe}(1)-\mathrm{C}(40)$ & $138.97(8)$ \\
\hline $\mathrm{C}(45)-\mathrm{Fe}(1)-\mathrm{C}(40)$ & $110.94(9)$ \\
\hline $\mathrm{C}(43)-\mathrm{Fe}(1)-\mathrm{C}(40)$ & $68.91(9)$ \\
\hline $\mathrm{C}(44)-\mathrm{Fe}(1)-\mathrm{C}(39)$ & $111.04(8)$ \\
\hline $\mathrm{C}(45)-\mathrm{Fe}(1)-\mathrm{C}(39)$ & $111.66(8)$ \\
\hline $\mathrm{C}(43)-\mathrm{Fe}(1)-\mathrm{C}(39)$ & $41.28(8)$ \\
\hline $\mathrm{C}(40)-\mathrm{Fe}(1)-\mathrm{C}(39)$ & $41.22(8)$ \\
\hline $\mathrm{C}(44)-\mathrm{Fe}(1)-\mathrm{C}(42)$ & $140.28(9)$ \\
\hline $\mathrm{C}(45)-\mathrm{Fe}(1)-\mathrm{C}(42)$ & $178.23(9)$ \\
\hline $\mathrm{C}(43)-\mathrm{Fe}(1)-\mathrm{C}(42)$ & $40.67(9)$ \\
\hline $\mathrm{C}(40)-\mathrm{Fe}(1)-\mathrm{C}(42)$ & $68.36(9)$ \\
\hline $\mathrm{C}(39)-\mathrm{Fe}(1)-\mathrm{C}(42)$ & $68.98(9)$ \\
\hline $\mathrm{C}(44)-\mathrm{Fe}(1)-\mathrm{C}(48)$ & $41.18(8)$ \\
\hline $\mathrm{C}(45)-\mathrm{Fe}(1)-\mathrm{C}(48)$ & $68.97(9)$ \\
\hline $\mathrm{C}(43)-\mathrm{Fe}(1)-\mathrm{C}(48)$ & $111.39(9)$ \\
\hline $\mathrm{C}(40)-\mathrm{Fe}(1)-\mathrm{C}(48)$ & $179.63(9)$ \\
\hline $\mathrm{C}(39)-\mathrm{Fe}(1)-\mathrm{C}(48)$ & $139.14(9)$ \\
\hline $\mathrm{C}(42)-\mathrm{Fe}(1)-\mathrm{C}(48)$ & $111.72(9)$ \\
\hline $\mathrm{C}(44)-\mathrm{Fe}(1)-\mathrm{C}(46)$ & $68.94(9)$ \\
\hline $\mathrm{C}(45)-\mathrm{Fe}(1)-\mathrm{C}(46)$ & $40.81(8)$ \\
\hline $\mathrm{C}(43)-\mathrm{Fe}(1)-\mathrm{C}(46)$ & $178.26(8)$ \\
\hline $\mathrm{C}(40)-\mathrm{Fe}(1)-\mathrm{C}(46)$ & $111.39(9)$ \\
\hline $\mathrm{C}(39)-\mathrm{Fe}(1)-\mathrm{C}(46)$ & $140.01(9)$ \\
\hline $\mathrm{C}(42)-\mathrm{Fe}(1)-\mathrm{C}(46)$ & $137.68(9)$ \\
\hline
\end{tabular}




\begin{tabular}{|c|c|}
\hline $\mathrm{C}(48)-\mathrm{Fe}(1)-\mathrm{C}(46)$ & $68.30(9)$ \\
\hline $\mathrm{C}(44)-\mathrm{Fe}(1)-\mathrm{C}(41)$ & $179.37(9)$ \\
\hline $\mathrm{C}(45)-\mathrm{Fe}(1)-\mathrm{C}(41)$ & $138.13(9)$ \\
\hline $\mathrm{C}(43)-\mathrm{Fe}(1)-\mathrm{C}(41)$ & $68.31(9)$ \\
\hline $\mathrm{C}(40)-\mathrm{Fe}(1)-\mathrm{C}(41)$ & $40.55(9)$ \\
\hline $\mathrm{C}(39)-\mathrm{Fe}(1)-\mathrm{C}(41)$ & $68.84(9)$ \\
\hline $\mathrm{C}(42)-\mathrm{Fe}(1)-\mathrm{C}(41)$ & $40.32(10)$ \\
\hline $\mathrm{C}(48)-\mathrm{Fe}(1)-\mathrm{C}(41)$ & $139.29(9)$ \\
\hline $\mathrm{C}(46)-\mathrm{Fe}(1)-\mathrm{C}(41)$ & $110.72(9)$ \\
\hline $\mathrm{C}(44)-\mathrm{Fe}(1)-\mathrm{C}(47)$ & $68.60(9)$ \\
\hline $\mathrm{C}(45)-\mathrm{Fe}(1)-\mathrm{C}(47)$ & $68.39(9)$ \\
\hline $\mathrm{C}(43)-\mathrm{Fe}(1)-\mathrm{C}(47)$ & $138.34(9)$ \\
\hline $\mathrm{C}(40)-\mathrm{Fe}(1)-\mathrm{C}(47)$ & 139.33(9) \\
\hline $\mathrm{C}(39)-\mathrm{Fe}(1)-\mathrm{C}(47)$ & $179.43(9)$ \\
\hline $\mathrm{C}(42)-\mathrm{Fe}(1)-\mathrm{C}(47)$ & $110.99(9)$ \\
\hline $\mathrm{C}(48)-\mathrm{Fe}(1)-\mathrm{C}(47)$ & $40.30(9)$ \\
\hline $\mathrm{C}(46)-\mathrm{Fe}(1)-\mathrm{C}(47)$ & $40.37(9)$ \\
\hline $\mathrm{C}(41)-\mathrm{Fe}(1)-\mathrm{C}(47)$ & $111.52(9)$ \\
\hline C(21)-P(1)-C(39) & $102.00(9)$ \\
\hline $\mathrm{C}(21)-\mathrm{P}(1)-\mathrm{C}(15)$ & 107.17(9) \\
\hline C(39)-P(1)-C(15) & 98.71(9) \\
\hline $\mathrm{C}(21)-\mathrm{P}(1)-\mathrm{Ni}(1)$ & $113.87(7)$ \\
\hline $\mathrm{C}(39)-\mathrm{P}(1)-\mathrm{Ni}(1)$ & $118.81(7)$ \\
\hline $\mathrm{C}(15)-\mathrm{P}(1)-\mathrm{Ni}(1)$ & $114.42(7)$ \\
\hline $\mathrm{C}(44)-\mathrm{P}(2)-\mathrm{C}(33)$ & $104.80(9)$ \\
\hline $\mathrm{C}(44)-\mathrm{P}(2)-\mathrm{C}(27)$ & $103.70(10)$ \\
\hline $\mathrm{C}(33)-\mathrm{P}(2)-\mathrm{C}(27)$ & $103.25(9)$ \\
\hline $\mathrm{C}(44)-\mathrm{P}(2)-\mathrm{Ni}(1)$ & $112.02(7)$ \\
\hline $\mathrm{C}(33)-\mathrm{P}(2)-\mathrm{Ni}(1)$ & $117.21(7)$ \\
\hline $\mathrm{C}(27)-\mathrm{P}(2)-\mathrm{Ni}(1)$ & $114.45(7)$ \\
\hline $\mathrm{C}(1)-\mathrm{O}(1)-\mathrm{Ni}(1)$ & $70.08(10)$ \\
\hline $\mathrm{O}(1)-\mathrm{C}(1)-\mathrm{C}(2)$ & $135.07(19)$ \\
\hline $\mathrm{O}(1)-\mathrm{C}(1)-\mathrm{Ni}(1)$ & $69.32(11)$ \\
\hline $\mathrm{C}(2)-\mathrm{C}(1)-\mathrm{Ni}(1)$ & $155.34(16)$ \\
\hline $\mathrm{C}(1)-\mathrm{C}(2)-\mathrm{C}(9)$ & $121.98(19)$ \\
\hline$C(1)-C(2)-C(3)$ & $120.16(18)$ \\
\hline
\end{tabular}




\begin{tabular}{|c|c|}
\hline $\mathrm{C}(9)-\mathrm{C}(2)-\mathrm{C}(3)$ & $117.84(17)$ \\
\hline$C(8)-C(3)-C(4)$ & $117.8(2)$ \\
\hline$C(8)-C(3)-C(2)$ & $122.62(19)$ \\
\hline$C(4)-C(3)-C(2)$ & $119.5(2)$ \\
\hline$C(5)-C(4)-C(3)$ & $121.3(2)$ \\
\hline $\mathrm{C}(5)-\mathrm{C}(4)-\mathrm{H}(4)$ & 119.4 \\
\hline $\mathrm{C}(3)-\mathrm{C}(4)-\mathrm{H}(4)$ & 119.4 \\
\hline$C(4)-C(5)-C(6)$ & $120.2(2)$ \\
\hline $\mathrm{C}(4)-\mathrm{C}(5)-\mathrm{H}(5)$ & 119.9 \\
\hline $\mathrm{C}(6)-\mathrm{C}(5)-\mathrm{H}(5)$ & 119.9 \\
\hline$C(7)-C(6)-C(5)$ & $119.4(2)$ \\
\hline $\mathrm{C}(7)-\mathrm{C}(6)-\mathrm{H}(6)$ & 120.3 \\
\hline $\mathrm{C}(5)-\mathrm{C}(6)-\mathrm{H}(6)$ & 120.3 \\
\hline$C(6)-C(7)-C(8)$ & $120.2(3)$ \\
\hline $\mathrm{C}(6)-\mathrm{C}(7)-\mathrm{H}(7)$ & 119.9 \\
\hline $\mathrm{C}(8)-\mathrm{C}(7)-\mathrm{H}(7)$ & 119.9 \\
\hline$C(3)-C(8)-C(7)$ & $121.0(2)$ \\
\hline $\mathrm{C}(3)-\mathrm{C}(8)-\mathrm{H}(8)$ & 119.5 \\
\hline $\mathrm{C}(7)-\mathrm{C}(8)-\mathrm{H}(8)$ & 119.5 \\
\hline$C(14)-C(9)-C(10)$ & $116.78(19)$ \\
\hline $\mathrm{C}(14)-\mathrm{C}(9)-\mathrm{C}(2)$ & $122.41(19)$ \\
\hline $\mathrm{C}(10)-\mathrm{C}(9)-\mathrm{C}(2)$ & $120.8(2)$ \\
\hline $\mathrm{C}(11)-\mathrm{C}(10)-\mathrm{C}(9)$ & $121.1(2)$ \\
\hline $\mathrm{C}(11)-\mathrm{C}(10)-\mathrm{H}(10)$ & 119.5 \\
\hline $\mathrm{C}(9)-\mathrm{C}(10)-\mathrm{H}(10)$ & 119.5 \\
\hline $\mathrm{C}(12)-\mathrm{C}(11)-\mathrm{C}(10)$ & $121.0(2)$ \\
\hline $\mathrm{C}(12)-\mathrm{C}(11)-\mathrm{H}(11)$ & 119.5 \\
\hline $\mathrm{C}(10)-\mathrm{C}(11)-\mathrm{H}(11)$ & 119.5 \\
\hline$C(11)-C(12)-C(13)$ & $119.0(2)$ \\
\hline $\mathrm{C}(11)-\mathrm{C}(12)-\mathrm{H}(12)$ & 120.5 \\
\hline $\mathrm{C}(13)-\mathrm{C}(12)-\mathrm{H}(12)$ & 120.5 \\
\hline$C(12)-C(13)-C(14)$ & $120.5(2)$ \\
\hline $\mathrm{C}(12)-\mathrm{C}(13)-\mathrm{H}(13)$ & 119.8 \\
\hline $\mathrm{C}(14)-\mathrm{C}(13)-\mathrm{H}(13)$ & 119.8 \\
\hline $\mathrm{C}(9)-\mathrm{C}(14)-\mathrm{C}(13)$ & $121.8(2)$ \\
\hline $\mathrm{C}(9)-\mathrm{C}(14)-\mathrm{H}(14)$ & 119.1 \\
\hline
\end{tabular}




\begin{tabular}{|c|c|}
\hline $\mathrm{C}(13)-\mathrm{C}(14)-\mathrm{H}(14)$ & 119.1 \\
\hline $\mathrm{C}(20)-\mathrm{C}(15)-\mathrm{C}(16)$ & $119.10(19)$ \\
\hline $\mathrm{C}(20)-\mathrm{C}(15)-\mathrm{P}(1)$ & $124.08(16)$ \\
\hline $\mathrm{C}(16)-\mathrm{C}(15)-\mathrm{P}(1)$ & $116.59(16)$ \\
\hline $\mathrm{C}(17)-\mathrm{C}(16)-\mathrm{C}(15)$ & $120.3(2)$ \\
\hline $\mathrm{C}(17)-\mathrm{C}(16)-\mathrm{H}(16)$ & 119.9 \\
\hline $\mathrm{C}(15)-\mathrm{C}(16)-\mathrm{H}(16)$ & 119.9 \\
\hline $\mathrm{C}(18)-\mathrm{C}(17)-\mathrm{C}(16)$ & $120.3(2)$ \\
\hline $\mathrm{C}(18)-\mathrm{C}(17)-\mathrm{H}(17)$ & 119.8 \\
\hline $\mathrm{C}(16)-\mathrm{C}(17)-\mathrm{H}(17)$ & 119.8 \\
\hline $\mathrm{C}(17)-\mathrm{C}(18)-\mathrm{C}(19)$ & $119.7(2)$ \\
\hline $\mathrm{C}(17)-\mathrm{C}(18)-\mathrm{H}(18)$ & 120.1 \\
\hline C(19)-C(18)-H(18) & 120.1 \\
\hline$C(18)-C(19)-C(20)$ & $120.3(2)$ \\
\hline $\mathrm{C}(18)-\mathrm{C}(19)-\mathrm{H}(19)$ & 119.9 \\
\hline $\mathrm{C}(20)-\mathrm{C}(19)-\mathrm{H}(19)$ & 119.9 \\
\hline $\mathrm{C}(15)-\mathrm{C}(20)-\mathrm{C}(19)$ & $120.3(2)$ \\
\hline $\mathrm{C}(15)-\mathrm{C}(20)-\mathrm{H}(20)$ & 119.9 \\
\hline $\mathrm{C}(19)-\mathrm{C}(20)-\mathrm{H}(20)$ & 119.9 \\
\hline$C(26)-C(21)-C(22)$ & $118.6(2)$ \\
\hline $\mathrm{C}(26)-\mathrm{C}(21)-\mathrm{P}(1)$ & $118.55(16)$ \\
\hline $\mathrm{C}(22)-\mathrm{C}(21)-\mathrm{P}(1)$ & $122.70(16)$ \\
\hline$C(23)-C(22)-C(21)$ & $120.5(2)$ \\
\hline $\mathrm{C}(23)-\mathrm{C}(22)-\mathrm{H}(22)$ & 119.8 \\
\hline $\mathrm{C}(21)-\mathrm{C}(22)-\mathrm{H}(22)$ & 119.8 \\
\hline$C(24)-C(23)-C(22)$ & $120.2(2)$ \\
\hline $\mathrm{C}(24)-\mathrm{C}(23)-\mathrm{H}(23)$ & 119.9 \\
\hline $\mathrm{C}(22)-\mathrm{C}(23)-\mathrm{H}(23)$ & 119.9 \\
\hline $\mathrm{C}(23)-\mathrm{C}(24)-\mathrm{C}(25)$ & $120.1(2)$ \\
\hline $\mathrm{C}(23)-\mathrm{C}(24)-\mathrm{H}(24)$ & 120.0 \\
\hline $\mathrm{C}(25)-\mathrm{C}(24)-\mathrm{H}(24)$ & 120.0 \\
\hline $\mathrm{C}(24)-\mathrm{C}(25)-\mathrm{C}(26)$ & $119.8(2)$ \\
\hline $\mathrm{C}(24)-\mathrm{C}(25)-\mathrm{H}(25)$ & 120.1 \\
\hline $\mathrm{C}(26)-\mathrm{C}(25)-\mathrm{H}(25)$ & 120.1 \\
\hline$C(25)-C(26)-C(21)$ & $120.8(2)$ \\
\hline $\mathrm{C}(25)-\mathrm{C}(26)-\mathrm{H}(26)$ & 119.6 \\
\hline
\end{tabular}




\begin{tabular}{|c|c|}
\hline $\mathrm{C}(21)-\mathrm{C}(26)-\mathrm{H}(26)$ & 119.6 \\
\hline $\mathrm{C}(32)-\mathrm{C}(27)-\mathrm{C}(28)$ & $119.0(2)$ \\
\hline $\mathrm{C}(32)-\mathrm{C}(27)-\mathrm{P}(2)$ & $122.73(18)$ \\
\hline $\mathrm{C}(28)-\mathrm{C}(27)-\mathrm{P}(2)$ & 118.31(18) \\
\hline $\mathrm{C}(29)-\mathrm{C}(28)-\mathrm{C}(27)$ & $120.6(2)$ \\
\hline $\mathrm{C}(29)-\mathrm{C}(28)-\mathrm{H}(28)$ & 119.7 \\
\hline $\mathrm{C}(27)-\mathrm{C}(28)-\mathrm{H}(28)$ & 119.7 \\
\hline $\mathrm{C}(30)-\mathrm{C}(29)-\mathrm{C}(28)$ & $119.9(2)$ \\
\hline $\mathrm{C}(30)-\mathrm{C}(29)-\mathrm{H}(29)$ & 120.1 \\
\hline $\mathrm{C}(28)-\mathrm{C}(29)-\mathrm{H}(29)$ & 120.1 \\
\hline $\mathrm{C}(29)-\mathrm{C}(30)-\mathrm{C}(31)$ & $120.4(2)$ \\
\hline $\mathrm{C}(29)-\mathrm{C}(30)-\mathrm{H}(30)$ & 119.8 \\
\hline $\mathrm{C}(31)-\mathrm{C}(30)-\mathrm{H}(30)$ & 119.8 \\
\hline $\mathrm{C}(30)-\mathrm{C}(31)-\mathrm{C}(32)$ & $119.8(3)$ \\
\hline $\mathrm{C}(30)-\mathrm{C}(31)-\mathrm{H}(31)$ & 120.1 \\
\hline $\mathrm{C}(32)-\mathrm{C}(31)-\mathrm{H}(31)$ & 120.1 \\
\hline $\mathrm{C}(27)-\mathrm{C}(32)-\mathrm{C}(31)$ & $120.4(2)$ \\
\hline $\mathrm{C}(27)-\mathrm{C}(32)-\mathrm{H}(32)$ & 119.8 \\
\hline $\mathrm{C}(31)-\mathrm{C}(32)-\mathrm{H}(32)$ & 119.8 \\
\hline $\mathrm{C}(34)-\mathrm{C}(33)-\mathrm{C}(38)$ & $119.1(2)$ \\
\hline $\mathrm{C}(34)-\mathrm{C}(33)-\mathrm{P}(2)$ & $118.30(16)$ \\
\hline $\mathrm{C}(38)-\mathrm{C}(33)-\mathrm{P}(2)$ & $122.57(17)$ \\
\hline $\mathrm{C}(33)-\mathrm{C}(34)-\mathrm{C}(35)$ & $120.9(2)$ \\
\hline $\mathrm{C}(33)-\mathrm{C}(34)-\mathrm{H}(34)$ & 119.6 \\
\hline $\mathrm{C}(35)-\mathrm{C}(34)-\mathrm{H}(34)$ & 119.6 \\
\hline $\mathrm{C}(36)-\mathrm{C}(35)-\mathrm{C}(34)$ & $119.7(2)$ \\
\hline $\mathrm{C}(36)-\mathrm{C}(35)-\mathrm{H}(35)$ & 120.2 \\
\hline $\mathrm{C}(34)-\mathrm{C}(35)-\mathrm{H}(35)$ & 120.2 \\
\hline $\mathrm{C}(37)-\mathrm{C}(36)-\mathrm{C}(35)$ & $119.9(2)$ \\
\hline $\mathrm{C}(37)-\mathrm{C}(36)-\mathrm{H}(36)$ & 120.1 \\
\hline $\mathrm{C}(35)-\mathrm{C}(36)-\mathrm{H}(36)$ & 120.1 \\
\hline $\mathrm{C}(36)-\mathrm{C}(37)-\mathrm{C}(38)$ & $120.7(2)$ \\
\hline $\mathrm{C}(36)-\mathrm{C}(37)-\mathrm{H}(37)$ & 119.6 \\
\hline $\mathrm{C}(38)-\mathrm{C}(37)-\mathrm{H}(37)$ & 119.6 \\
\hline $\mathrm{C}(37)-\mathrm{C}(38)-\mathrm{C}(33)$ & $119.7(2)$ \\
\hline $\mathrm{C}(37)-\mathrm{C}(38)-\mathrm{H}(38)$ & 120.2 \\
\hline
\end{tabular}




\begin{tabular}{|c|c|}
\hline $\mathrm{C}(33)-\mathrm{C}(38)-\mathrm{H}(38)$ & 120.2 \\
\hline$C(40)-C(39)-C(43)$ & $106.60(18)$ \\
\hline $\mathrm{C}(40)-\mathrm{C}(39)-\mathrm{P}(1)$ & $126.99(16)$ \\
\hline $\mathrm{C}(43)-\mathrm{C}(39)-\mathrm{P}(1)$ & $126.38(16)$ \\
\hline $\mathrm{C}(40)-\mathrm{C}(39)-\mathrm{Fe}(1)$ & $69.27(12)$ \\
\hline $\mathrm{C}(43)-\mathrm{C}(39)-\mathrm{Fe}(1)$ & $69.02(13)$ \\
\hline $\mathrm{P}(1)-\mathrm{C}(39)-\mathrm{Fe}(1)$ & $125.05(10)$ \\
\hline $\mathrm{C}(41)-\mathrm{C}(40)-\mathrm{C}(39)$ & $108.3(2)$ \\
\hline $\mathrm{C}(41)-\mathrm{C}(40)-\mathrm{Fe}(1)$ & $70.16(13)$ \\
\hline $\mathrm{C}(39)-\mathrm{C}(40)-\mathrm{Fe}(1)$ & $69.51(12)$ \\
\hline $\mathrm{C}(41)-\mathrm{C}(40)-\mathrm{H}(40)$ & 125.8 \\
\hline $\mathrm{C}(39)-\mathrm{C}(40)-\mathrm{H}(40)$ & 125.8 \\
\hline $\mathrm{Fe}(1)-\mathrm{C}(40)-\mathrm{H}(40)$ & 126.1 \\
\hline $\mathrm{C}(42)-\mathrm{C}(41)-\mathrm{C}(40)$ & $108.4(2)$ \\
\hline $\mathrm{C}(42)-\mathrm{C}(41)-\mathrm{Fe}(1)$ & $69.63(12)$ \\
\hline $\mathrm{C}(40)-\mathrm{C}(41)-\mathrm{Fe}(1)$ & $69.28(12)$ \\
\hline $\mathrm{C}(42)-\mathrm{C}(41)-\mathrm{H}(41)$ & 125.8 \\
\hline $\mathrm{C}(40)-\mathrm{C}(41)-\mathrm{H}(41)$ & 125.8 \\
\hline $\mathrm{Fe}(1)-\mathrm{C}(41)-\mathrm{H}(41)$ & 126.9 \\
\hline $\mathrm{C}(41)-\mathrm{C}(42)-\mathrm{C}(43)$ & $108.3(2)$ \\
\hline $\mathrm{C}(41)-\mathrm{C}(42)-\mathrm{Fe}(1)$ & $70.06(13)$ \\
\hline $\mathrm{C}(43)-\mathrm{C}(42)-\mathrm{Fe}(1)$ & $69.22(12)$ \\
\hline $\mathrm{C}(41)-\mathrm{C}(42)-\mathrm{H}(42)$ & 125.8 \\
\hline $\mathrm{C}(43)-\mathrm{C}(42)-\mathrm{H}(42)$ & 125.8 \\
\hline $\mathrm{Fe}(1)-\mathrm{C}(42)-\mathrm{H}(42)$ & 126.5 \\
\hline $\mathrm{C}(42)-\mathrm{C}(43)-\mathrm{C}(39)$ & $108.4(2)$ \\
\hline $\mathrm{C}(42)-\mathrm{C}(43)-\mathrm{Fe}(1)$ & $70.11(13)$ \\
\hline $\mathrm{C}(39)-\mathrm{C}(43)-\mathrm{Fe}(1)$ & $69.70(12)$ \\
\hline $\mathrm{C}(42)-\mathrm{C}(43)-\mathrm{H}(43)$ & 125.8 \\
\hline $\mathrm{C}(39)-\mathrm{C}(43)-\mathrm{H}(43)$ & 125.8 \\
\hline $\mathrm{Fe}(1)-\mathrm{C}(43)-\mathrm{H}(43)$ & 126.0 \\
\hline$C(45)-C(44)-C(48)$ & $107.52(18)$ \\
\hline $\mathrm{C}(45)-\mathrm{C}(44)-\mathrm{P}(2)$ & $121.53(15)$ \\
\hline $\mathrm{C}(48)-\mathrm{C}(44)-\mathrm{P}(2)$ & $130.70(16)$ \\
\hline $\mathrm{C}(45)-\mathrm{C}(44)-\mathrm{Fe}(1)$ & $69.52(11)$ \\
\hline $\mathrm{C}(48)-\mathrm{C}(44)-\mathrm{Fe}(1)$ & $70.18(12)$ \\
\hline
\end{tabular}




$\begin{array}{lc}\mathrm{P}(2)-\mathrm{C}(44)-\mathrm{Fe}(1) & 121.06(11) \\ \mathrm{C}(46)-\mathrm{C}(45)-\mathrm{C}(44) & 107.94(18) \\ \mathrm{C}(46)-\mathrm{C}(45)-\mathrm{Fe}(1) & 70.29(12) \\ \mathrm{C}(44)-\mathrm{C}(45)-\mathrm{Fe}(1) & 69.21(11) \\ \mathrm{C}(46)-\mathrm{C}(45)-\mathrm{H}(45) & 126.0 \\ \mathrm{C}(44)-\mathrm{C}(45)-\mathrm{H}(45) & 126.0 \\ \mathrm{Fe}(1)-\mathrm{C}(45)-\mathrm{H}(45) & 126.0 \\ \mathrm{C}(47)-\mathrm{C}(46)-\mathrm{C}(45) & 108.06(19) \\ \mathrm{C}(47)-\mathrm{C}(46)-\mathrm{Fe}(1) & 70.20(13) \\ \mathrm{C}(45)-\mathrm{C}(46)-\mathrm{Fe}(1) & 68.90(12) \\ \mathrm{C}(47)-\mathrm{C}(46)-\mathrm{H}(46) & 126.0 \\ \mathrm{C}(45)-\mathrm{C}(46)-\mathrm{H}(46) & 126.0 \\ \mathrm{Fe}(1)-\mathrm{C}(46)-\mathrm{H}(46) & 126.5 \\ \mathrm{C}(48)-\mathrm{C}(47)-\mathrm{C}(46) & 108.55(19) \\ \mathrm{C}(48)-\mathrm{C}(47)-\mathrm{Fe}(1) & 69.39(13) \\ \mathrm{C}(46)-\mathrm{C}(47)-\mathrm{Fe}(1) & 69.43(13) \\ \mathrm{C}(48)-\mathrm{C}(47)-\mathrm{H}(47) & 125.7 \\ \mathrm{C}(46)-\mathrm{C}(47)-\mathrm{H}(47) & 125.7 \\ \mathrm{Fe}(1)-\mathrm{C}(47)-\mathrm{H}(47) & 127.0 \\ \mathrm{C}(47)-\mathrm{C}(48)-\mathrm{C}(44) & 107.92(19) \\ \mathrm{C}(47)-\mathrm{C}(48)-\mathrm{Fe}(1) & 70.30(13) \\ \mathrm{C}(44)-\mathrm{C}(48)-\mathrm{Fe}(1) & 68.63(12) \\ \mathrm{C}(47)-\mathrm{C}(48)-\mathrm{H}(48) & 126.0 \\ \mathrm{C}(44)-\mathrm{C}(48)-\mathrm{H}(48) & 126.0 \\ \mathrm{Fe}(1)-\mathrm{C}(48)-\mathrm{H}(48) & 126.6 \\ & \\ & \end{array}$

Symmetry transformations used to generate equivalent atoms: 
Table S4d. Anisotropic displacement parameters $\left(\AA^{2} \times 10^{3}\right)$ for 8a. The anisotropic displacement factor exponent takes the form: $-2 \pi^{2}\left[h^{2} a^{* 2} U^{11}+\ldots+2 h k a^{*} b^{*} U^{12}\right]$

\begin{tabular}{|c|c|c|c|c|c|c|}
\hline & $\mathrm{U}^{11}$ & $\mathrm{U}^{22}$ & $\mathrm{U}^{33}$ & $\mathrm{U}^{23}$ & $\mathrm{U}^{13}$ & $\mathrm{U}^{12}$ \\
\hline $\mathrm{Ni}(1)$ & $16(1)$ & $17(1)$ & $16(1)$ & $1(1)$ & $3(1)$ & $0(1)$ \\
\hline $\mathrm{Fe}(1)$ & $21(1)$ & $22(1)$ & $17(1)$ & $2(1)$ & $2(1)$ & $1(1)$ \\
\hline $\mathrm{P}(1)$ & $17(1)$ & $20(1)$ & $17(1)$ & $1(1)$ & $4(1)$ & $0(1)$ \\
\hline $\mathrm{P}(2)$ & $20(1)$ & $18(1)$ & $19(1)$ & $2(1)$ & $4(1)$ & $0(1)$ \\
\hline $\mathrm{O}(1)$ & $18(1)$ & $21(1)$ & $20(1)$ & $3(1)$ & $2(1)$ & $-1(1)$ \\
\hline$C(1)$ & $18(1)$ & $20(1)$ & $19(1)$ & $-4(1)$ & $4(1)$ & $-1(1)$ \\
\hline$C(2)$ & $23(1)$ & $18(1)$ & $18(1)$ & $0(1)$ & $4(1)$ & $2(1)$ \\
\hline$C(3)$ & $23(1)$ & $23(1)$ & $20(1)$ & $5(1)$ & $3(1)$ & $2(1)$ \\
\hline$C(4)$ & $28(1)$ & $24(1)$ & $30(1)$ & $3(1)$ & $5(1)$ & $2(1)$ \\
\hline$C(5)$ & $36(1)$ & $29(1)$ & $38(1)$ & $6(1)$ & $4(1)$ & $-6(1)$ \\
\hline$C(6)$ & $39(1)$ & $52(2)$ & $37(2)$ & $12(1)$ & 11(1) & $-14(1)$ \\
\hline$C(7)$ & $40(2)$ & $56(2)$ & $40(2)$ & $0(1)$ & $21(1)$ & $-2(1)$ \\
\hline$C(8)$ & $35(1)$ & $30(1)$ & $32(1)$ & $-2(1)$ & $14(1)$ & $1(1)$ \\
\hline$C(9)$ & $25(1)$ & $21(1)$ & $19(1)$ & $-1(1)$ & $2(1)$ & $1(1)$ \\
\hline$C(10)$ & $29(1)$ & $30(1)$ & $24(1)$ & $5(1)$ & $3(1)$ & $0(1)$ \\
\hline $\mathrm{C}(11)$ & $38(1)$ & $33(1)$ & $31(1)$ & $11(1)$ & $1(1)$ & $8(1)$ \\
\hline$C(12)$ & $27(1)$ & $45(2)$ & $36(1)$ & $8(1)$ & $1(1)$ & $12(1)$ \\
\hline$C(13)$ & $27(1)$ & $48(2)$ & $38(2)$ & $11(1)$ & $12(1)$ & $6(1)$ \\
\hline$C(14)$ & $26(1)$ & $36(1)$ & $28(1)$ & $10(1)$ & $6(1)$ & $5(1)$ \\
\hline$C(15)$ & $19(1)$ & $21(1)$ & $21(1)$ & $-1(1)$ & $3(1)$ & $0(1)$ \\
\hline$C(16)$ & $23(1)$ & $27(1)$ & $27(1)$ & $-1(1)$ & $7(1)$ & $0(1)$ \\
\hline$C(17)$ & $30(1)$ & $29(1)$ & $30(1)$ & $-9(1)$ & $5(1)$ & $3(1)$ \\
\hline $\mathrm{C}(18)$ & $29(1)$ & $23(1)$ & $39(1)$ & $-5(1)$ & $-1(1)$ & $-4(1)$ \\
\hline C(19) & $25(1)$ & $27(1)$ & $42(1)$ & $2(1)$ & $8(1)$ & $-6(1)$ \\
\hline $\mathrm{C}(20)$ & $20(1)$ & $25(1)$ & $29(1)$ & $1(1)$ & $9(1)$ & $1(1)$ \\
\hline $\mathrm{C}(21)$ & $22(1)$ & $16(1)$ & $22(1)$ & $3(1)$ & $9(1)$ & $3(1)$ \\
\hline $\mathrm{C}(22)$ & $20(1)$ & $24(1)$ & $30(1)$ & $0(1)$ & $7(1)$ & $0(1)$ \\
\hline$C(23)$ & $23(1)$ & $29(1)$ & $45(2)$ & $2(1)$ & $15(1)$ & $4(1)$ \\
\hline $\mathrm{C}(24)$ & $39(1)$ & $34(1)$ & $40(2)$ & $-2(1)$ & $24(1)$ & $7(1)$ \\
\hline$C(25)$ & $36(1)$ & $40(1)$ & $25(1)$ & $-7(1)$ & $12(1)$ & $3(1)$ \\
\hline$C(26)$ & $23(1)$ & $29(1)$ & $24(1)$ & $0(1)$ & $7(1)$ & $2(1)$ \\
\hline
\end{tabular}




\begin{tabular}{lllllll}
$\mathrm{C}(27)$ & $22(1)$ & $18(1)$ & $31(1)$ & $3(1)$ & $8(1)$ & $1(1)$ \\
$\mathrm{C}(28)$ & $26(1)$ & $26(1)$ & $33(1)$ & $6(1)$ & $2(1)$ & $-1(1)$ \\
$\mathrm{C}(29)$ & $26(1)$ & $30(1)$ & $51(2)$ & $7(1)$ & $-2(1)$ & $-2(1)$ \\
$\mathrm{C}(30)$ & $23(1)$ & $33(1)$ & $76(2)$ & $3(1)$ & $12(1)$ & $-2(1)$ \\
$\mathrm{C}(31)$ & $41(2)$ & $50(2)$ & $66(2)$ & $-2(2)$ & $33(2)$ & $-9(1)$ \\
$\mathrm{C}(32)$ & $34(1)$ & $42(1)$ & $39(2)$ & $-4(1)$ & $14(1)$ & $-9(1)$ \\
$\mathrm{C}(33)$ & $21(1)$ & $21(1)$ & $22(1)$ & $-2(1)$ & $2(1)$ & $0(1)$ \\
$\mathrm{C}(34)$ & $31(1)$ & $23(1)$ & $27(1)$ & $-3(1)$ & $7(1)$ & $-2(1)$ \\
$\mathrm{C}(35)$ & $32(1)$ & $38(1)$ & $35(1)$ & $-13(1)$ & $10(1)$ & $0(1)$ \\
$\mathrm{C}(36)$ & $39(1)$ & $30(1)$ & $38(2)$ & $-8(1)$ & $6(1)$ & $12(1)$ \\
$\mathrm{C}(37)$ & $47(2)$ & $21(1)$ & $38(1)$ & $2(1)$ & $7(1)$ & $5(1)$ \\
$\mathrm{C}(38)$ & $31(1)$ & $24(1)$ & $31(1)$ & $2(1)$ & $8(1)$ & $1(1)$ \\
$\mathrm{C}(39)$ & $16(1)$ & $27(1)$ & $20(1)$ & $0(1)$ & $4(1)$ & $-2(1)$ \\
$\mathrm{C}(40)$ & $23(1)$ & $28(1)$ & $23(1)$ & $-1(1)$ & $4(1)$ & $-7(1)$ \\
$\mathrm{C}(41)$ & $23(1)$ & $44(1)$ & $22(1)$ & $1(1)$ & $0(1)$ & $-8(1)$ \\
$\mathrm{C}(42)$ & $21(1)$ & $40(1)$ & $28(1)$ & $6(1)$ & $2(1)$ & $6(1)$ \\
$\mathrm{C}(43)$ & $21(1)$ & $30(1)$ & $24(1)$ & $1(1)$ & $5(1)$ & $4(1)$ \\
$\mathrm{C}(44)$ & $21(1)$ & $21(1)$ & $20(1)$ & $3(1)$ & $6(1)$ & $-2(1)$ \\
$\mathrm{C}(45)$ & $22(1)$ & $24(1)$ & $21(1)$ & $1(1)$ & $6(1)$ & $3(1)$ \\
$\mathrm{C}(46)$ & $32(1)$ & $29(1)$ & $21(1)$ & $-1(1)$ & $11(1)$ & $2(1)$ \\
$\mathrm{C}(47)$ & $32(1)$ & $34(1)$ & $21(1)$ & $6(1)$ & $7(1)$ & $0(1)$ \\
$\mathrm{C}(48)$ & $30(1)$ & $24(1)$ & $23(1)$ & $6(1)$ & $6(1)$ & $1(1)$ \\
& & & & & & \\
\hline
\end{tabular}


Table S4e. Hydrogen coordinates $\left(\mathrm{x} 10^{4}\right)$ and isotropic displacement parameters $\left(\AA^{2} \times 10^{3}\right)$ for $\mathbf{8 a}$.

\begin{tabular}{|c|c|c|c|c|}
\hline & $\mathrm{x}$ & $\mathrm{y}$ & $\mathrm{z}$ & $\mathrm{U}(\mathrm{eq})$ \\
\hline $\mathrm{H}(4)$ & 5307 & -551 & 1658 & 34 \\
\hline $\mathrm{H}(5)$ & 3740 & -1188 & 1965 & 42 \\
\hline $\mathrm{H}(6)$ & 2451 & -678 & 2619 & 51 \\
\hline $\mathrm{H}(7)$ & 2789 & 469 & 2993 & 52 \\
\hline $\mathrm{H}(8)$ & 4420 & 1101 & 2724 & 38 \\
\hline $\mathrm{H}(10)$ & 7042 & -120 & 3100 & 35 \\
\hline $\mathrm{H}(11)$ & 9116 & -517 & 3607 & 43 \\
\hline $\mathrm{H}(12)$ & 10751 & -140 & 3136 & 45 \\
\hline $\mathrm{H}(13)$ & 10289 & 632 & 2132 & 45 \\
\hline $\mathrm{H}(14)$ & 8214 & 1019 & 1603 & 36 \\
\hline $\mathrm{H}(16)$ & 4024 & 448 & -45 & 31 \\
\hline $\mathrm{H}(17)$ & 3757 & -735 & -271 & 37 \\
\hline $\mathrm{H}(18)$ & 2219 & -1341 & 117 & 39 \\
\hline $\mathrm{H}(19)$ & 916 & -759 & 714 & 38 \\
\hline $\mathrm{H}(20)$ & 1117 & 433 & 898 & 29 \\
\hline $\mathrm{H}(22)$ & 71 & 1639 & 468 & 29 \\
\hline $\mathrm{H}(23)$ & -969 & 2088 & 1300 & 37 \\
\hline $\mathrm{H}(24)$ & 206 & 2530 & 2457 & 42 \\
\hline $\mathrm{H}(25)$ & 2430 & 2526 & 2790 & 39 \\
\hline $\mathrm{H}(26)$ & 3478 & 2083 & 1960 & 30 \\
\hline $\mathrm{H}(28)$ & 6793 & 3251 & 1174 & 35 \\
\hline $\mathrm{H}(29)$ & 8821 & 3684 & 1254 & 46 \\
\hline $\mathrm{H}(30)$ & 9510 & 3740 & 188 & 53 \\
\hline $\mathrm{H}(31)$ & 8157 & 3409 & -976 & 59 \\
\hline $\mathrm{H}(32)$ & 6093 & 3017 & -1067 & 45 \\
\hline $\mathrm{H}(34)$ & 3043 & 3063 & 921 & 33 \\
\hline $\mathrm{H}(35)$ & 1783 & 3991 & 1076 & 42 \\
\hline $\mathrm{H}(36)$ & 1829 & 5022 & 436 & 44 \\
\hline $\mathrm{H}(37)$ & 3169 & 5131 & -328 & 44 \\
\hline $\mathrm{H}(38)$ & 4397 & 4199 & -509 & 35 \\
\hline
\end{tabular}




\begin{tabular}{rrrrr}
$\mathrm{H}(40)$ & 1494 & 800 & -1088 & 30 \\
$\mathrm{H}(41)$ & 25 & 1478 & -2154 & 37 \\
$\mathrm{H}(42)$ & -160 & 2689 & -1699 & 37 \\
$\mathrm{H}(43)$ & 1179 & 2774 & -341 & 30 \\
$\mathrm{H}(45)$ & 4514 & 1491 & -961 & 27 \\
$\mathrm{H}(46)$ & 3169 & 1456 & -2326 & 32 \\
$\mathrm{H}(47)$ & 2201 & 2639 & -2675 & 35 \\
$\mathrm{H}(48)$ & 2923 & 3413 & -1535 & 31 \\
\hline
\end{tabular}


Table S4f. Torsion angles $\left[{ }^{\circ}\right]$ for $8 \mathbf{a}$.

\begin{tabular}{|c|c|}
\hline $\mathrm{O}(1)-\mathrm{Ni}(1)-\mathrm{P}(1)-\mathrm{C}(21)$ & $95.82(12)$ \\
\hline $\mathrm{C}(1)-\mathrm{Ni}(1)-\mathrm{P}(1)-\mathrm{C}(21)$ & $85.57(10)$ \\
\hline $\mathrm{P}(2)-\mathrm{Ni}(1)-\mathrm{P}(1)-\mathrm{C}(21)$ & $-98.21(7)$ \\
\hline $\mathrm{O}(1)-\mathrm{Ni}(1)-\mathrm{P}(1)-\mathrm{C}(39)$ & $-143.98(12)$ \\
\hline $\mathrm{C}(1)-\mathrm{Ni}(1)-\mathrm{P}(1)-\mathrm{C}(39)$ & $-154.24(10)$ \\
\hline $\mathrm{P}(2)-\mathrm{Ni}(1)-\mathrm{P}(1)-\mathrm{C}(39)$ & $21.98(8)$ \\
\hline $\mathrm{O}(1)-\mathrm{Ni}(1)-\mathrm{P}(1)-\mathrm{C}(15)$ & $-27.92(13)$ \\
\hline $\mathrm{C}(1)-\mathrm{Ni}(1)-\mathrm{P}(1)-\mathrm{C}(15)$ & $-38.17(10)$ \\
\hline $\mathrm{P}(2)-\mathrm{Ni}(1)-\mathrm{P}(1)-\mathrm{C}(15)$ & $138.05(8)$ \\
\hline $\mathrm{O}(1)-\mathrm{Ni}(1)-\mathrm{P}(2)-\mathrm{C}(44)$ & 118.91(9) \\
\hline $\mathrm{C}(1)-\mathrm{Ni}(1)-\mathrm{P}(2)-\mathrm{C}(44)$ & 119.98(13) \\
\hline $\mathrm{P}(1)-\mathrm{Ni}(1)-\mathrm{P}(2)-\mathrm{C}(44)$ & $-54.01(8)$ \\
\hline $\mathrm{O}(1)-\mathrm{Ni}(1)-\mathrm{P}(2)-\mathrm{C}(33)$ & $-119.91(9)$ \\
\hline $\mathrm{C}(1)-\mathrm{Ni}(1)-\mathrm{P}(2)-\mathrm{C}(33)$ & $-118.83(13)$ \\
\hline $\mathrm{P}(1)-\mathrm{Ni}(1)-\mathrm{P}(2)-\mathrm{C}(33)$ & $67.17(8)$ \\
\hline $\mathrm{O}(1)-\mathrm{Ni}(1)-\mathrm{P}(2)-\mathrm{C}(27)$ & $1.22(9)$ \\
\hline $\mathrm{C}(1)-\mathrm{Ni}(1)-\mathrm{P}(2)-\mathrm{C}(27)$ & $2.30(14)$ \\
\hline $\mathrm{P}(1)-\mathrm{Ni}(1)-\mathrm{P}(2)-\mathrm{C}(27)$ & $-171.70(8)$ \\
\hline $\mathrm{P}(1)-\mathrm{Ni}(1)-\mathrm{O}(1)-\mathrm{C}(1)$ & $-14.86(17)$ \\
\hline $\mathrm{P}(2)-\mathrm{Ni}(1)-\mathrm{O}(1)-\mathrm{C}(1)$ & $179.03(11)$ \\
\hline $\mathrm{Ni}(1)-\mathrm{O}(1)-\mathrm{C}(1)-\mathrm{C}(2)$ & $175.6(3)$ \\
\hline $\mathrm{P}(1)-\mathrm{Ni}(1)-\mathrm{C}(1)-\mathrm{O}(1)$ & $172.22(9)$ \\
\hline $\mathrm{P}(2)-\mathrm{Ni}(1)-\mathrm{C}(1)-\mathrm{O}(1)$ & $-1.61(17)$ \\
\hline $\mathrm{O}(1)-\mathrm{Ni}(1)-\mathrm{C}(1)-\mathrm{C}(2)$ & $-172.6(5)$ \\
\hline $\mathrm{P}(1)-\mathrm{Ni}(1)-\mathrm{C}(1)-\mathrm{C}(2)$ & $-0.3(4)$ \\
\hline $\mathrm{P}(2)-\mathrm{Ni}(1)-\mathrm{C}(1)-\mathrm{C}(2)$ & $-174.2(3)$ \\
\hline $\mathrm{O}(1)-\mathrm{C}(1)-\mathrm{C}(2)-\mathrm{C}(9)$ & $-4.6(4)$ \\
\hline $\mathrm{Ni}(1)-\mathrm{C}(1)-\mathrm{C}(2)-\mathrm{C}(9)$ & $165.6(3)$ \\
\hline $\mathrm{O}(1)-\mathrm{C}(1)-\mathrm{C}(2)-\mathrm{C}(3)$ & $176.7(2)$ \\
\hline $\mathrm{Ni}(1)-\mathrm{C}(1)-\mathrm{C}(2)-\mathrm{C}(3)$ & $-13.1(5)$ \\
\hline$C(1)-C(2)-C(3)-C(8)$ & $-66.3(3)$ \\
\hline $\mathrm{C}(9)-\mathrm{C}(2)-\mathrm{C}(3)-\mathrm{C}(8)$ & $114.9(2)$ \\
\hline$C(1)-C(2)-C(3)-C(4)$ & $115.5(2)$ \\
\hline$C(9)-C(2)-C(3)-C(4)$ & $-63.2(3)$ \\
\hline
\end{tabular}




\begin{tabular}{|c|c|}
\hline$C(8)-C(3)-C(4)-C(5)$ & $0.7(3)$ \\
\hline$C(2)-C(3)-C(4)-C(5)$ & $178.9(2)$ \\
\hline$C(3)-C(4)-C(5)-C(6)$ & $0.8(4)$ \\
\hline$C(4)-C(5)-C(6)-C(7)$ & $-1.1(4)$ \\
\hline$C(5)-C(6)-C(7)-C(8)$ & $-0.2(4)$ \\
\hline$C(4)-C(3)-C(8)-C(7)$ & $-2.0(3)$ \\
\hline$C(2)-C(3)-C(8)-C(7)$ & $179.8(2)$ \\
\hline$C(6)-C(7)-C(8)-C(3)$ & $1.8(4)$ \\
\hline$C(1)-C(2)-C(9)-C(14)$ & $-14.3(3)$ \\
\hline$C(3)-C(2)-C(9)-C(14)$ & $164.4(2)$ \\
\hline$C(1)-C(2)-C(9)-C(10)$ & $166.0(2)$ \\
\hline$C(3)-C(2)-C(9)-C(10)$ & $-15.2(3)$ \\
\hline$C(14)-C(9)-C(10)-C(11)$ & $-0.5(3)$ \\
\hline$C(2)-C(9)-C(10)-C(11)$ & $179.2(2)$ \\
\hline $\mathrm{C}(9)-\mathrm{C}(10)-\mathrm{C}(11)-\mathrm{C}(12)$ & $0.8(4)$ \\
\hline $\mathrm{C}(10)-\mathrm{C}(11)-\mathrm{C}(12)-\mathrm{C}(13)$ & $-0.5(4)$ \\
\hline $\mathrm{C}(11)-\mathrm{C}(12)-\mathrm{C}(13)-\mathrm{C}(14)$ & $-0.1(4)$ \\
\hline $\mathrm{C}(10)-\mathrm{C}(9)-\mathrm{C}(14)-\mathrm{C}(13)$ & $-0.1(3)$ \\
\hline$C(2)-C(9)-C(14)-C(13)$ & $-179.8(2)$ \\
\hline $\mathrm{C}(12)-\mathrm{C}(13)-\mathrm{C}(14)-\mathrm{C}(9)$ & $0.4(4)$ \\
\hline $\mathrm{C}(21)-\mathrm{P}(1)-\mathrm{C}(15)-\mathrm{C}(20)$ & $17.5(2)$ \\
\hline $\mathrm{C}(39)-\mathrm{P}(1)-\mathrm{C}(15)-\mathrm{C}(20)$ & $-88.03(19)$ \\
\hline $\mathrm{Ni}(1)-\mathrm{P}(1)-\mathrm{C}(15)-\mathrm{C}(20)$ & $144.75(16)$ \\
\hline $\mathrm{C}(21)-\mathrm{P}(1)-\mathrm{C}(15)-\mathrm{C}(16)$ & $-168.08(16)$ \\
\hline $\mathrm{C}(39)-\mathrm{P}(1)-\mathrm{C}(15)-\mathrm{C}(16)$ & $86.41(17)$ \\
\hline $\mathrm{Ni}(1)-\mathrm{P}(1)-\mathrm{C}(15)-\mathrm{C}(16)$ & $-40.81(18)$ \\
\hline$C(20)-C(15)-C(16)-C(17)$ & $1.1(3)$ \\
\hline $\mathrm{P}(1)-\mathrm{C}(15)-\mathrm{C}(16)-\mathrm{C}(17)$ & $-173.68(17)$ \\
\hline $\mathrm{C}(15)-\mathrm{C}(16)-\mathrm{C}(17)-\mathrm{C}(18)$ & $-1.5(3)$ \\
\hline $\mathrm{C}(16)-\mathrm{C}(17)-\mathrm{C}(18)-\mathrm{C}(19)$ & $0.6(3)$ \\
\hline $\mathrm{C}(17)-\mathrm{C}(18)-\mathrm{C}(19)-\mathrm{C}(20)$ & $0.8(4)$ \\
\hline$C(16)-C(15)-C(20)-C(19)$ & $0.4(3)$ \\
\hline $\mathrm{P}(1)-\mathrm{C}(15)-\mathrm{C}(20)-\mathrm{C}(19)$ & $174.66(17)$ \\
\hline $\mathrm{C}(18)-\mathrm{C}(19)-\mathrm{C}(20)-\mathrm{C}(15)$ & $-1.3(3)$ \\
\hline $\mathrm{C}(39)-\mathrm{P}(1)-\mathrm{C}(21)-\mathrm{C}(26)$ & $-145.59(17)$ \\
\hline$C(15)-P(1)-C(21)-C(26)$ & $111.25(17)$ \\
\hline
\end{tabular}




\begin{tabular}{|c|c|}
\hline $\mathrm{Ni}(1)-\mathrm{P}(1)-\mathrm{C}(21)-\mathrm{C}(26)$ & $-16.33(18)$ \\
\hline $\mathrm{C}(39)-\mathrm{P}(1)-\mathrm{C}(21)-\mathrm{C}(22)$ & $30.46(19)$ \\
\hline $\mathrm{C}(15)-\mathrm{P}(1)-\mathrm{C}(21)-\mathrm{C}(22)$ & $-72.71(19)$ \\
\hline Ni(1)-P(1)-C(21)-C(22) & $159.71(15)$ \\
\hline$C(26)-C(21)-C(22)-C(23)$ & $-1.0(3)$ \\
\hline $\mathrm{P}(1)-\mathrm{C}(21)-\mathrm{C}(22)-\mathrm{C}(23)$ & $-177.05(17)$ \\
\hline$C(21)-C(22)-C(23)-C(24)$ & $0.4(3)$ \\
\hline$C(22)-C(23)-C(24)-C(25)$ & $0.1(4)$ \\
\hline$C(23)-C(24)-C(25)-C(26)$ & $0.0(4)$ \\
\hline$C(24)-C(25)-C(26)-C(21)$ & $-0.7(4)$ \\
\hline$C(22)-C(21)-C(26)-C(25)$ & $1.2(3)$ \\
\hline $\mathrm{P}(1)-\mathrm{C}(21)-\mathrm{C}(26)-\mathrm{C}(25)$ & $177.36(17)$ \\
\hline $\mathrm{C}(44)-\mathrm{P}(2)-\mathrm{C}(27)-\mathrm{C}(32)$ & $0.9(2)$ \\
\hline $\mathrm{C}(33)-\mathrm{P}(2)-\mathrm{C}(27)-\mathrm{C}(32)$ & $-108.2(2)$ \\
\hline Ni(1)-P(2)-C(27)-C(32) & $123.24(18)$ \\
\hline $\mathrm{C}(44)-\mathrm{P}(2)-\mathrm{C}(27)-\mathrm{C}(28)$ & $-178.38(17)$ \\
\hline $\mathrm{C}(33)-\mathrm{P}(2)-\mathrm{C}(27)-\mathrm{C}(28)$ & $72.50(18)$ \\
\hline $\mathrm{Ni}(1)-\mathrm{P}(2)-\mathrm{C}(27)-\mathrm{C}(28)$ & $-56.04(18)$ \\
\hline $\mathrm{C}(32)-\mathrm{C}(27)-\mathrm{C}(28)-\mathrm{C}(29)$ & $0.3(3)$ \\
\hline $\mathrm{P}(2)-\mathrm{C}(27)-\mathrm{C}(28)-\mathrm{C}(29)$ & $179.60(17)$ \\
\hline $\mathrm{C}(27)-\mathrm{C}(28)-\mathrm{C}(29)-\mathrm{C}(30)$ & $-1.5(3)$ \\
\hline $\mathrm{C}(28)-\mathrm{C}(29)-\mathrm{C}(30)-\mathrm{C}(31)$ & $1.3(4)$ \\
\hline $\mathrm{C}(29)-\mathrm{C}(30)-\mathrm{C}(31)-\mathrm{C}(32)$ & $0.0(4)$ \\
\hline $\mathrm{C}(28)-\mathrm{C}(27)-\mathrm{C}(32)-\mathrm{C}(31)$ & $1.1(4)$ \\
\hline $\mathrm{P}(2)-\mathrm{C}(27)-\mathrm{C}(32)-\mathrm{C}(31)$ & $-178.2(2)$ \\
\hline $\mathrm{C}(30)-\mathrm{C}(31)-\mathrm{C}(32)-\mathrm{C}(27)$ & $-1.2(4)$ \\
\hline $\mathrm{C}(44)-\mathrm{P}(2)-\mathrm{C}(33)-\mathrm{C}(34)$ & $113.92(17)$ \\
\hline $\mathrm{C}(27)-\mathrm{P}(2)-\mathrm{C}(33)-\mathrm{C}(34)$ & $-137.78(17)$ \\
\hline $\mathrm{Ni}(1)-\mathrm{P}(2)-\mathrm{C}(33)-\mathrm{C}(34)$ & $-10.97(19)$ \\
\hline $\mathrm{C}(44)-\mathrm{P}(2)-\mathrm{C}(33)-\mathrm{C}(38)$ & $-63.79(19)$ \\
\hline $\mathrm{C}(27)-\mathrm{P}(2)-\mathrm{C}(33)-\mathrm{C}(38)$ & $44.5(2)$ \\
\hline Ni(1)-P(2)-C(33)-C(38) & $171.32(15)$ \\
\hline $\mathrm{C}(38)-\mathrm{C}(33)-\mathrm{C}(34)-\mathrm{C}(35)$ & $2.6(3)$ \\
\hline $\mathrm{P}(2)-\mathrm{C}(33)-\mathrm{C}(34)-\mathrm{C}(35)$ & $-175.18(17)$ \\
\hline $\mathrm{C}(33)-\mathrm{C}(34)-\mathrm{C}(35)-\mathrm{C}(36)$ & $-1.2(3)$ \\
\hline $\mathrm{C}(34)-\mathrm{C}(35)-\mathrm{C}(36)-\mathrm{C}(37)$ & $-1.0(4)$ \\
\hline
\end{tabular}




\begin{tabular}{|c|c|}
\hline $\mathrm{C}(35)-\mathrm{C}(36)-\mathrm{C}(37)-\mathrm{C}(38)$ & $1.8(4)$ \\
\hline $\mathrm{C}(36)-\mathrm{C}(37)-\mathrm{C}(38)-\mathrm{C}(33)$ & $-0.4(4)$ \\
\hline $\mathrm{C}(34)-\mathrm{C}(33)-\mathrm{C}(38)-\mathrm{C}(37)$ & $-1.8(3)$ \\
\hline $\mathrm{P}(2)-\mathrm{C}(33)-\mathrm{C}(38)-\mathrm{C}(37)$ & $175.87(17)$ \\
\hline $\mathrm{C}(21)-\mathrm{P}(1)-\mathrm{C}(39)-\mathrm{C}(40)$ & $-131.34(19)$ \\
\hline $\mathrm{C}(15)-\mathrm{P}(1)-\mathrm{C}(39)-\mathrm{C}(40)$ & $-21.6(2)$ \\
\hline $\mathrm{Ni}(1)-\mathrm{P}(1)-\mathrm{C}(39)-\mathrm{C}(40)$ & $102.56(18)$ \\
\hline $\mathrm{C}(21)-\mathrm{P}(1)-\mathrm{C}(39)-\mathrm{C}(43)$ & $50.9(2)$ \\
\hline $\mathrm{C}(15)-\mathrm{P}(1)-\mathrm{C}(39)-\mathrm{C}(43)$ & $160.67(19)$ \\
\hline $\mathrm{Ni}(1)-\mathrm{P}(1)-\mathrm{C}(39)-\mathrm{C}(43)$ & $-75.2(2)$ \\
\hline $\mathrm{C}(21)-\mathrm{P}(1)-\mathrm{C}(39)-\mathrm{Fe}(1)$ & $139.40(13)$ \\
\hline $\mathrm{C}(15)-\mathrm{P}(1)-\mathrm{C}(39)-\mathrm{Fe}(1)$ & $-110.85(13)$ \\
\hline $\mathrm{Ni}(1)-\mathrm{P}(1)-\mathrm{C}(39)-\mathrm{Fe}(1)$ & $13.31(16)$ \\
\hline $\mathrm{C}(44)-\mathrm{Fe}(1)-\mathrm{C}(39)-\mathrm{C}(40)$ & $-141.95(12)$ \\
\hline $\mathrm{C}(45)-\mathrm{Fe}(1)-\mathrm{C}(39)-\mathrm{C}(40)$ & $-97.49(13)$ \\
\hline $\mathrm{C}(43)-\mathrm{Fe}(1)-\mathrm{C}(39)-\mathrm{C}(40)$ & $118.19(17)$ \\
\hline $\mathrm{C}(42)-\mathrm{Fe}(1)-\mathrm{C}(39)-\mathrm{C}(40)$ & $80.74(13)$ \\
\hline $\mathrm{C}(48)-\mathrm{Fe}(1)-\mathrm{C}(39)-\mathrm{C}(40)$ & $-179.96(13)$ \\
\hline $\mathrm{C}(46)-\mathrm{Fe}(1)-\mathrm{C}(39)-\mathrm{C}(40)$ & $-60.02(17)$ \\
\hline $\mathrm{C}(41)-\mathrm{Fe}(1)-\mathrm{C}(39)-\mathrm{C}(40)$ & $37.38(13)$ \\
\hline $\mathrm{C}(47)-\mathrm{Fe}(1)-\mathrm{C}(39)-\mathrm{C}(40)$ & $167(23)$ \\
\hline $\mathrm{C}(44)-\mathrm{Fe}(1)-\mathrm{C}(39)-\mathrm{C}(43)$ & $99.86(13)$ \\
\hline $\mathrm{C}(45)-\mathrm{Fe}(1)-\mathrm{C}(39)-\mathrm{C}(43)$ & $144.33(12)$ \\
\hline $\mathrm{C}(40)-\mathrm{Fe}(1)-\mathrm{C}(39)-\mathrm{C}(43)$ & $-118.19(17)$ \\
\hline $\mathrm{C}(42)-\mathrm{Fe}(1)-\mathrm{C}(39)-\mathrm{C}(43)$ & $-37.45(12)$ \\
\hline $\mathrm{C}(48)-\mathrm{Fe}(1)-\mathrm{C}(39)-\mathrm{C}(43)$ & $61.86(17)$ \\
\hline $\mathrm{C}(46)-\mathrm{Fe}(1)-\mathrm{C}(39)-\mathrm{C}(43)$ & $-178.21(13)$ \\
\hline $\mathrm{C}(41)-\mathrm{Fe}(1)-\mathrm{C}(39)-\mathrm{C}(43)$ & $-80.80(13)$ \\
\hline $\mathrm{C}(47)-\mathrm{Fe}(1)-\mathrm{C}(39)-\mathrm{C}(43)$ & $49(9)$ \\
\hline $\mathrm{C}(44)-\mathrm{Fe}(1)-\mathrm{C}(39)-\mathrm{P}(1)$ & $-20.60(16)$ \\
\hline $\mathrm{C}(45)-\mathrm{Fe}(1)-\mathrm{C}(39)-\mathrm{P}(1)$ & $23.87(16)$ \\
\hline $\mathrm{C}(43)-\mathrm{Fe}(1)-\mathrm{C}(39)-\mathrm{P}(1)$ & $-120.46(19)$ \\
\hline $\mathrm{C}(40)-\mathrm{Fe}(1)-\mathrm{C}(39)-\mathrm{P}(1)$ & $121.35(19)$ \\
\hline $\mathrm{C}(42)-\mathrm{Fe}(1)-\mathrm{C}(39)-\mathrm{P}(1)$ & $-157.91(16)$ \\
\hline $\mathrm{C}(48)-\mathrm{Fe}(1)-\mathrm{C}(39)-\mathrm{P}(1)$ & $-58.60(19)$ \\
\hline $\mathrm{C}(46)-\mathrm{Fe}(1)-\mathrm{C}(39)-\mathrm{P}(1)$ & $61.33(19)$ \\
\hline
\end{tabular}




\begin{tabular}{|c|c|}
\hline $\mathrm{C}(41)-\mathrm{Fe}(1)-\mathrm{C}(39)-\mathrm{P}(1)$ & $158.74(16)$ \\
\hline $\mathrm{C}(47)-\mathrm{Fe}(1)-\mathrm{C}(39)-\mathrm{P}(1)$ & $-71(9)$ \\
\hline$C(43)-C(39)-C(40)-C(41)$ & $-0.5(2)$ \\
\hline $\mathrm{P}(1)-\mathrm{C}(39)-\mathrm{C}(40)-\mathrm{C}(41)$ & $-178.57(16)$ \\
\hline $\mathrm{Fe}(1)-\mathrm{C}(39)-\mathrm{C}(40)-\mathrm{C}(41)$ & $-59.65(15)$ \\
\hline $\mathrm{C}(43)-\mathrm{C}(39)-\mathrm{C}(40)-\mathrm{Fe}(1)$ & $59.18(14)$ \\
\hline $\mathrm{P}(1)-\mathrm{C}(39)-\mathrm{C}(40)-\mathrm{Fe}(1)$ & $-118.92(17)$ \\
\hline $\mathrm{C}(44)-\mathrm{Fe}(1)-\mathrm{C}(40)-\mathrm{C}(41)$ & $-179.37(14)$ \\
\hline $\mathrm{C}(45)-\mathrm{Fe}(1)-\mathrm{C}(40)-\mathrm{C}(41)$ & $-141.19(14)$ \\
\hline $\mathrm{C}(43)-\mathrm{Fe}(1)-\mathrm{C}(40)-\mathrm{C}(41)$ & $80.88(15)$ \\
\hline $\mathrm{C}(39)-\mathrm{Fe}(1)-\mathrm{C}(40)-\mathrm{C}(41)$ & $119.44(19)$ \\
\hline $\mathrm{C}(42)-\mathrm{Fe}(1)-\mathrm{C}(40)-\mathrm{C}(41)$ & $37.06(14)$ \\
\hline $\mathrm{C}(48)-\mathrm{Fe}(1)-\mathrm{C}(40)-\mathrm{C}(41)$ & $-65(15)$ \\
\hline $\mathrm{C}(46)-\mathrm{Fe}(1)-\mathrm{C}(40)-\mathrm{C}(41)$ & $-97.28(15)$ \\
\hline $\mathrm{C}(47)-\mathrm{Fe}(1)-\mathrm{C}(40)-\mathrm{C}(41)$ & $-60.4(2)$ \\
\hline $\mathrm{C}(44)-\mathrm{Fe}(1)-\mathrm{C}(40)-\mathrm{C}(39)$ & $61.20(18)$ \\
\hline $\mathrm{C}(45)-\mathrm{Fe}(1)-\mathrm{C}(40)-\mathrm{C}(39)$ & $99.37(13)$ \\
\hline $\mathrm{C}(43)-\mathrm{Fe}(1)-\mathrm{C}(40)-\mathrm{C}(39)$ & $-38.56(12)$ \\
\hline $\mathrm{C}(42)-\mathrm{Fe}(1)-\mathrm{C}(40)-\mathrm{C}(39)$ & $-82.37(13)$ \\
\hline $\mathrm{C}(48)-\mathrm{Fe}(1)-\mathrm{C}(40)-\mathrm{C}(39)$ & $176(100)$ \\
\hline $\mathrm{C}(46)-\mathrm{Fe}(1)-\mathrm{C}(40)-\mathrm{C}(39)$ & $143.28(12)$ \\
\hline $\mathrm{C}(41)-\mathrm{Fe}(1)-\mathrm{C}(40)-\mathrm{C}(39)$ & $-119.44(19)$ \\
\hline $\mathrm{C}(47)-\mathrm{Fe}(1)-\mathrm{C}(40)-\mathrm{C}(39)$ & $-179.81(13)$ \\
\hline $\mathrm{C}(39)-\mathrm{C}(40)-\mathrm{C}(41)-\mathrm{C}(42)$ & $0.4(3)$ \\
\hline $\mathrm{Fe}(1)-\mathrm{C}(40)-\mathrm{C}(41)-\mathrm{C}(42)$ & $-58.80(16)$ \\
\hline $\mathrm{C}(39)-\mathrm{C}(40)-\mathrm{C}(41)-\mathrm{Fe}(1)$ & $59.24(15)$ \\
\hline $\mathrm{C}(44)-\mathrm{Fe}(1)-\mathrm{C}(41)-\mathrm{C}(42)$ & $161(24)$ \\
\hline $\mathrm{C}(45)-\mathrm{Fe}(1)-\mathrm{C}(41)-\mathrm{C}(42)$ & $-178.69(14)$ \\
\hline $\mathrm{C}(43)-\mathrm{Fe}(1)-\mathrm{C}(41)-\mathrm{C}(42)$ & $37.54(14)$ \\
\hline $\mathrm{C}(40)-\mathrm{Fe}(1)-\mathrm{C}(41)-\mathrm{C}(42)$ & $120.0(2)$ \\
\hline $\mathrm{C}(39)-\mathrm{Fe}(1)-\mathrm{C}(41)-\mathrm{C}(42)$ & $82.04(14)$ \\
\hline $\mathrm{C}(48)-\mathrm{Fe}(1)-\mathrm{C}(41)-\mathrm{C}(42)$ & $-60.5(2)$ \\
\hline $\mathrm{C}(46)-\mathrm{Fe}(1)-\mathrm{C}(41)-\mathrm{C}(42)$ & $-140.90(14)$ \\
\hline $\mathrm{C}(47)-\mathrm{Fe}(1)-\mathrm{C}(41)-\mathrm{C}(42)$ & $-97.49(15)$ \\
\hline $\mathrm{C}(44)-\mathrm{Fe}(1)-\mathrm{C}(41)-\mathrm{C}(40)$ & $41(8)$ \\
\hline $\mathrm{C}(45)-\mathrm{Fe}(1)-\mathrm{C}(41)-\mathrm{C}(40)$ & $61.29(19)$ \\
\hline
\end{tabular}




\begin{tabular}{|c|c|}
\hline $\mathrm{C}(43)-\mathrm{Fe}(1)-\mathrm{C}(41)-\mathrm{C}(40)$ & $-82.48(14)$ \\
\hline $\mathrm{C}(39)-\mathrm{Fe}(1)-\mathrm{C}(41)-\mathrm{C}(40)$ & $-37.98(13)$ \\
\hline $\mathrm{C}(42)-\mathrm{Fe}(1)-\mathrm{C}(41)-\mathrm{C}(40)$ & $-120.0(2)$ \\
\hline $\mathrm{C}(48)-\mathrm{Fe}(1)-\mathrm{C}(41)-\mathrm{C}(40)$ & $179.49(14)$ \\
\hline $\mathrm{C}(46)-\mathrm{Fe}(1)-\mathrm{C}(41)-\mathrm{C}(40)$ & $99.07(15)$ \\
\hline $\mathrm{C}(47)-\mathrm{Fe}(1)-\mathrm{C}(41)-\mathrm{C}(40)$ & $142.49(14)$ \\
\hline$C(40)-C(41)-C(42)-C(43)$ & $-0.2(3)$ \\
\hline $\mathrm{Fe}(1)-\mathrm{C}(41)-\mathrm{C}(42)-\mathrm{C}(43)$ & $-58.83(16)$ \\
\hline $\mathrm{C}(40)-\mathrm{C}(41)-\mathrm{C}(42)-\mathrm{Fe}(1)$ & $58.59(16)$ \\
\hline $\mathrm{C}(44)-\mathrm{Fe}(1)-\mathrm{C}(42)-\mathrm{C}(41)$ & $-179.68(14)$ \\
\hline $\mathrm{C}(45)-\mathrm{Fe}(1)-\mathrm{C}(42)-\mathrm{C}(41)$ & $30(3)$ \\
\hline $\mathrm{C}(43)-\mathrm{Fe}(1)-\mathrm{C}(42)-\mathrm{C}(41)$ & $-119.7(2)$ \\
\hline $\mathrm{C}(40)-\mathrm{Fe}(1)-\mathrm{C}(42)-\mathrm{C}(41)$ & $-37.27(13)$ \\
\hline $\mathrm{C}(39)-\mathrm{Fe}(1)-\mathrm{C}(42)-\mathrm{C}(41)$ & $-81.68(14)$ \\
\hline $\mathrm{C}(48)-\mathrm{Fe}(1)-\mathrm{C}(42)-\mathrm{C}(41)$ & $142.34(14)$ \\
\hline $\mathrm{C}(46)-\mathrm{Fe}(1)-\mathrm{C}(42)-\mathrm{C}(41)$ & $61.18(19)$ \\
\hline $\mathrm{C}(47)-\mathrm{Fe}(1)-\mathrm{C}(42)-\mathrm{C}(41)$ & $98.93(15)$ \\
\hline $\mathrm{C}(44)-\mathrm{Fe}(1)-\mathrm{C}(42)-\mathrm{C}(43)$ & $-60.00(19)$ \\
\hline $\mathrm{C}(45)-\mathrm{Fe}(1)-\mathrm{C}(42)-\mathrm{C}(43)$ & $149(3)$ \\
\hline $\mathrm{C}(40)-\mathrm{Fe}(1)-\mathrm{C}(42)-\mathrm{C}(43)$ & $82.40(14)$ \\
\hline $\mathrm{C}(39)-\mathrm{Fe}(1)-\mathrm{C}(42)-\mathrm{C}(43)$ & $38.00(13)$ \\
\hline $\mathrm{C}(48)-\mathrm{Fe}(1)-\mathrm{C}(42)-\mathrm{C}(43)$ & $-97.98(14)$ \\
\hline $\mathrm{C}(46)-\mathrm{Fe}(1)-\mathrm{C}(42)-\mathrm{C}(43)$ & $-179.14(14)$ \\
\hline $\mathrm{C}(41)-\mathrm{Fe}(1)-\mathrm{C}(42)-\mathrm{C}(43)$ & $119.7(2)$ \\
\hline $\mathrm{C}(47)-\mathrm{Fe}(1)-\mathrm{C}(42)-\mathrm{C}(43)$ & $-141.40(13)$ \\
\hline $\mathrm{C}(41)-\mathrm{C}(42)-\mathrm{C}(43)-\mathrm{C}(39)$ & $-0.1(3)$ \\
\hline $\mathrm{Fe}(1)-\mathrm{C}(42)-\mathrm{C}(43)-\mathrm{C}(39)$ & $-59.40(15)$ \\
\hline $\mathrm{C}(41)-\mathrm{C}(42)-\mathrm{C}(43)-\mathrm{Fe}(1)$ & $59.35(16)$ \\
\hline$C(40)-C(39)-C(43)-C(42)$ & $0.3(2)$ \\
\hline $\mathrm{P}(1)-\mathrm{C}(39)-\mathrm{C}(43)-\mathrm{C}(42)$ & $178.44(16)$ \\
\hline $\mathrm{Fe}(1)-\mathrm{C}(39)-\mathrm{C}(43)-\mathrm{C}(42)$ & $59.66(15)$ \\
\hline $\mathrm{C}(40)-\mathrm{C}(39)-\mathrm{C}(43)-\mathrm{Fe}(1)$ & $-59.34(14)$ \\
\hline $\mathrm{P}(1)-\mathrm{C}(39)-\mathrm{C}(43)-\mathrm{Fe}(1)$ & $118.78(16)$ \\
\hline $\mathrm{C}(44)-\mathrm{Fe}(1)-\mathrm{C}(43)-\mathrm{C}(42)$ & $143.34(13)$ \\
\hline $\mathrm{C}(45)-\mathrm{Fe}(1)-\mathrm{C}(43)-\mathrm{C}(42)$ & $-178.57(14)$ \\
\hline $\mathrm{C}(40)-\mathrm{Fe}(1)-\mathrm{C}(43)-\mathrm{C}(42)$ & $-80.93(14)$ \\
\hline
\end{tabular}




\begin{tabular}{|c|c|}
\hline $\mathrm{C}(39)-\mathrm{Fe}(1)-\mathrm{C}(43)-\mathrm{C}(42)$ & $-119.43(18)$ \\
\hline $\mathrm{C}(48)-\mathrm{Fe}(1)-\mathrm{C}(43)-\mathrm{C}(42)$ & $98.85(14)$ \\
\hline $\mathrm{C}(46)-\mathrm{Fe}(1)-\mathrm{C}(43)-\mathrm{C}(42)$ & 19(3) \\
\hline $\mathrm{C}(41)-\mathrm{Fe}(1)-\mathrm{C}(43)-\mathrm{C}(42)$ & $-37.23(14)$ \\
\hline $\mathrm{C}(47)-\mathrm{Fe}(1)-\mathrm{C}(43)-\mathrm{C}(42)$ & $61.22(19)$ \\
\hline $\mathrm{C}(44)-\mathrm{Fe}(1)-\mathrm{C}(43)-\mathrm{C}(39)$ & $-97.23(13)$ \\
\hline $\mathrm{C}(45)-\mathrm{Fe}(1)-\mathrm{C}(43)-\mathrm{C}(39)$ & $-59.14(18)$ \\
\hline $\mathrm{C}(40)-\mathrm{Fe}(1)-\mathrm{C}(43)-\mathrm{C}(39)$ & $38.50(12)$ \\
\hline $\mathrm{C}(42)-\mathrm{Fe}(1)-\mathrm{C}(43)-\mathrm{C}(39)$ & $119.43(18)$ \\
\hline $\mathrm{C}(48)-\mathrm{Fe}(1)-\mathrm{C}(43)-\mathrm{C}(39)$ & $-141.72(12)$ \\
\hline $\mathrm{C}(46)-\mathrm{Fe}(1)-\mathrm{C}(43)-\mathrm{C}(39)$ & $139(3)$ \\
\hline $\mathrm{C}(41)-\mathrm{Fe}(1)-\mathrm{C}(43)-\mathrm{C}(39)$ & $82.20(13)$ \\
\hline $\mathrm{C}(47)-\mathrm{Fe}(1)-\mathrm{C}(43)-\mathrm{C}(39)$ & $-179.36(13)$ \\
\hline $\mathrm{C}(33)-\mathrm{P}(2)-\mathrm{C}(44)-\mathrm{C}(45)$ & $-158.65(17)$ \\
\hline $\mathrm{C}(27)-\mathrm{P}(2)-\mathrm{C}(44)-\mathrm{C}(45)$ & $93.38(18)$ \\
\hline $\mathrm{Ni}(1)-\mathrm{P}(2)-\mathrm{C}(44)-\mathrm{C}(45)$ & $-30.55(19)$ \\
\hline $\mathrm{C}(33)-\mathrm{P}(2)-\mathrm{C}(44)-\mathrm{C}(48)$ & $14.8(2)$ \\
\hline $\mathrm{C}(27)-\mathrm{P}(2)-\mathrm{C}(44)-\mathrm{C}(48)$ & $-93.1(2)$ \\
\hline $\mathrm{Ni}(1)-\mathrm{P}(2)-\mathrm{C}(44)-\mathrm{C}(48)$ & $142.93(18)$ \\
\hline $\mathrm{C}(33)-\mathrm{P}(2)-\mathrm{C}(44)-\mathrm{Fe}(1)$ & $-74.95(13)$ \\
\hline $\mathrm{C}(27)-\mathrm{P}(2)-\mathrm{C}(44)-\mathrm{Fe}(1)$ & $177.08(11)$ \\
\hline $\mathrm{Ni}(1)-\mathrm{P}(2)-\mathrm{C}(44)-\mathrm{Fe}(1)$ & $53.15(13)$ \\
\hline $\mathrm{C}(43)-\mathrm{Fe}(1)-\mathrm{C}(44)-\mathrm{C}(45)$ & $143.81(12)$ \\
\hline $\mathrm{C}(40)-\mathrm{Fe}(1)-\mathrm{C}(44)-\mathrm{C}(45)$ & $61.06(18)$ \\
\hline $\mathrm{C}(39)-\mathrm{Fe}(1)-\mathrm{C}(44)-\mathrm{C}(45)$ & $99.28(13)$ \\
\hline $\mathrm{C}(42)-\mathrm{Fe}(1)-\mathrm{C}(44)-\mathrm{C}(45)$ & $-178.68(14)$ \\
\hline $\mathrm{C}(48)-\mathrm{Fe}(1)-\mathrm{C}(44)-\mathrm{C}(45)$ & $-118.44(18)$ \\
\hline $\mathrm{C}(46)-\mathrm{Fe}(1)-\mathrm{C}(44)-\mathrm{C}(45)$ & $-37.74(12)$ \\
\hline $\mathrm{C}(41)-\mathrm{Fe}(1)-\mathrm{C}(44)-\mathrm{C}(45)$ & $20(8)$ \\
\hline $\mathrm{C}(47)-\mathrm{Fe}(1)-\mathrm{C}(44)-\mathrm{C}(45)$ & $-81.19(13)$ \\
\hline $\mathrm{C}(45)-\mathrm{Fe}(1)-\mathrm{C}(44)-\mathrm{C}(48)$ & $118.44(18)$ \\
\hline $\mathrm{C}(43)-\mathrm{Fe}(1)-\mathrm{C}(44)-\mathrm{C}(48)$ & $-97.76(14)$ \\
\hline $\mathrm{C}(40)-\mathrm{Fe}(1)-\mathrm{C}(44)-\mathrm{C}(48)$ & $179.49(14)$ \\
\hline $\mathrm{C}(39)-\mathrm{Fe}(1)-\mathrm{C}(44)-\mathrm{C}(48)$ & $-142.29(13)$ \\
\hline $\mathrm{C}(42)-\mathrm{Fe}(1)-\mathrm{C}(44)-\mathrm{C}(48)$ & $-60.25(19)$ \\
\hline $\mathrm{C}(46)-\mathrm{Fe}(1)-\mathrm{C}(44)-\mathrm{C}(48)$ & $80.70(14)$ \\
\hline
\end{tabular}




\begin{tabular}{|c|c|}
\hline $\mathrm{C}(41)-\mathrm{Fe}(1)-\mathrm{C}(44)-\mathrm{C}(48)$ & $139(8)$ \\
\hline $\mathrm{C}(47)-\mathrm{Fe}(1)-\mathrm{C}(44)-\mathrm{C}(48)$ & $37.24(13)$ \\
\hline $\mathrm{C}(45)-\mathrm{Fe}(1)-\mathrm{C}(44)-\mathrm{P}(2)$ & $-115.25(18)$ \\
\hline $\mathrm{C}(43)-\mathrm{Fe}(1)-\mathrm{C}(44)-\mathrm{P}(2)$ & $28.56(15)$ \\
\hline $\mathrm{C}(40)-\mathrm{Fe}(1)-\mathrm{C}(44)-\mathrm{P}(2)$ & $-54.20(19)$ \\
\hline $\mathrm{C}(39)-\mathrm{Fe}(1)-\mathrm{C}(44)-\mathrm{P}(2)$ & $-15.98(15)$ \\
\hline $\mathrm{C}(42)-\mathrm{Fe}(1)-\mathrm{C}(44)-\mathrm{P}(2)$ & $66.06(18)$ \\
\hline $\mathrm{C}(48)-\mathrm{Fe}(1)-\mathrm{C}(44)-\mathrm{P}(2)$ & $126.31(19)$ \\
\hline $\mathrm{C}(46)-\mathrm{Fe}(1)-\mathrm{C}(44)-\mathrm{P}(2)$ & $-152.99(15)$ \\
\hline $\mathrm{C}(41)-\mathrm{Fe}(1)-\mathrm{C}(44)-\mathrm{P}(2)$ & $-95(8)$ \\
\hline $\mathrm{C}(47)-\mathrm{Fe}(1)-\mathrm{C}(44)-\mathrm{P}(2)$ & $163.55(15)$ \\
\hline $\mathrm{C}(48)-\mathrm{C}(44)-\mathrm{C}(45)-\mathrm{C}(46)$ & $-0.3(2)$ \\
\hline $\mathrm{P}(2)-\mathrm{C}(44)-\mathrm{C}(45)-\mathrm{C}(46)$ & $174.51(15)$ \\
\hline $\mathrm{Fe}(1)-\mathrm{C}(44)-\mathrm{C}(45)-\mathrm{C}(46)$ & $59.86(15)$ \\
\hline $\mathrm{C}(48)-\mathrm{C}(44)-\mathrm{C}(45)-\mathrm{Fe}(1)$ & $-60.17(14)$ \\
\hline $\mathrm{P}(2)-\mathrm{C}(44)-\mathrm{C}(45)-\mathrm{Fe}(1)$ & $114.65(15)$ \\
\hline $\mathrm{C}(44)-\mathrm{Fe}(1)-\mathrm{C}(45)-\mathrm{C}(46)$ & $-119.08(18)$ \\
\hline $\mathrm{C}(43)-\mathrm{Fe}(1)-\mathrm{C}(45)-\mathrm{C}(46)$ & $-179.18(14)$ \\
\hline $\mathrm{C}(40)-\mathrm{Fe}(1)-\mathrm{C}(45)-\mathrm{C}(46)$ & $98.88(14)$ \\
\hline $\mathrm{C}(39)-\mathrm{Fe}(1)-\mathrm{C}(45)-\mathrm{C}(46)$ & $143.27(13)$ \\
\hline $\mathrm{C}(42)-\mathrm{Fe}(1)-\mathrm{C}(45)-\mathrm{C}(46)$ & $33(3)$ \\
\hline $\mathrm{C}(48)-\mathrm{Fe}(1)-\mathrm{C}(45)-\mathrm{C}(46)$ & $-80.74(14)$ \\
\hline $\mathrm{C}(41)-\mathrm{Fe}(1)-\mathrm{C}(45)-\mathrm{C}(46)$ & $61.25(18)$ \\
\hline $\mathrm{C}(47)-\mathrm{Fe}(1)-\mathrm{C}(45)-\mathrm{C}(46)$ & $-37.33(13)$ \\
\hline $\mathrm{C}(43)-\mathrm{Fe}(1)-\mathrm{C}(45)-\mathrm{C}(44)$ & $-60.10(18)$ \\
\hline $\mathrm{C}(40)-\mathrm{Fe}(1)-\mathrm{C}(45)-\mathrm{C}(44)$ & $-142.04(12)$ \\
\hline $\mathrm{C}(39)-\mathrm{Fe}(1)-\mathrm{C}(45)-\mathrm{C}(44)$ & $-97.65(13)$ \\
\hline $\mathrm{C}(42)-\mathrm{Fe}(1)-\mathrm{C}(45)-\mathrm{C}(44)$ & $152(3)$ \\
\hline $\mathrm{C}(48)-\mathrm{Fe}(1)-\mathrm{C}(45)-\mathrm{C}(44)$ & $38.34(12)$ \\
\hline $\mathrm{C}(46)-\mathrm{Fe}(1)-\mathrm{C}(45)-\mathrm{C}(44)$ & $119.08(18)$ \\
\hline $\mathrm{C}(41)-\mathrm{Fe}(1)-\mathrm{C}(45)-\mathrm{C}(44)$ & $-179.67(13)$ \\
\hline $\mathrm{C}(47)-\mathrm{Fe}(1)-\mathrm{C}(45)-\mathrm{C}(44)$ & $81.74(13)$ \\
\hline$C(44)-C(45)-C(46)-C(47)$ & $0.3(2)$ \\
\hline $\mathrm{Fe}(1)-\mathrm{C}(45)-\mathrm{C}(46)-\mathrm{C}(47)$ & $59.48(16)$ \\
\hline $\mathrm{C}(44)-\mathrm{C}(45)-\mathrm{C}(46)-\mathrm{Fe}(1)$ & $-59.18(14)$ \\
\hline $\mathrm{C}(44)-\mathrm{Fe}(1)-\mathrm{C}(46)-\mathrm{C}(47)$ & $-81.33(14)$ \\
\hline
\end{tabular}


$\mathrm{C}(45)-\mathrm{Fe}(1)-\mathrm{C}(46)-\mathrm{C}(47)$

$\mathrm{C}(43)-\mathrm{Fe}(1)-\mathrm{C}(46)-\mathrm{C}(47)$

$\mathrm{C}(40)-\mathrm{Fe}(1)-\mathrm{C}(46)-\mathrm{C}(47)$

$\mathrm{C}(39)-\mathrm{Fe}(1)-\mathrm{C}(46)-\mathrm{C}(47)$

$\mathrm{C}(42)-\mathrm{Fe}(1)-\mathrm{C}(46)-\mathrm{C}(47)$

$\mathrm{C}(48)-\mathrm{Fe}(1)-\mathrm{C}(46)-\mathrm{C}(47)$

$\mathrm{C}(41)-\mathrm{Fe}(1)-\mathrm{C}(46)-\mathrm{C}(47)$

$\mathrm{C}(44)-\mathrm{Fe}(1)-\mathrm{C}(46)-\mathrm{C}(45)$

$\mathrm{C}(43)-\mathrm{Fe}(1)-\mathrm{C}(46)-\mathrm{C}(45)$

$\mathrm{C}(40)-\mathrm{Fe}(1)-\mathrm{C}(46)-\mathrm{C}(45)$

$\mathrm{C}(39)-\mathrm{Fe}(1)-\mathrm{C}(46)-\mathrm{C}(45)$

$\mathrm{C}(42)-\mathrm{Fe}(1)-\mathrm{C}(46)-\mathrm{C}(45)$

$\mathrm{C}(48)-\mathrm{Fe}(1)-\mathrm{C}(46)-\mathrm{C}(45)$

$\mathrm{C}(41)-\mathrm{Fe}(1)-\mathrm{C}(46)-\mathrm{C}(45)$

$\mathrm{C}(47)-\mathrm{Fe}(1)-\mathrm{C}(46)-\mathrm{C}(45)$

$\mathrm{C}(45)-\mathrm{C}(46)-\mathrm{C}(47)-\mathrm{C}(48)$

$\mathrm{Fe}(1)-\mathrm{C}(46)-\mathrm{C}(47)-\mathrm{C}(48)$

$\mathrm{C}(45)-\mathrm{C}(46)-\mathrm{C}(47)-\mathrm{Fe}(1)$

$\mathrm{C}(44)-\mathrm{Fe}(1)-\mathrm{C}(47)-\mathrm{C}(48)$

$\mathrm{C}(45)-\mathrm{Fe}(1)-\mathrm{C}(47)-\mathrm{C}(48)$

$\mathrm{C}(43)-\mathrm{Fe}(1)-\mathrm{C}(47)-\mathrm{C}(48)$

$\mathrm{C}(40)-\mathrm{Fe}(1)-\mathrm{C}(47)-\mathrm{C}(48)$

$\mathrm{C}(39)-\mathrm{Fe}(1)-\mathrm{C}(47)-\mathrm{C}(48)$

$\mathrm{C}(42)-\mathrm{Fe}(1)-\mathrm{C}(47)-\mathrm{C}(48)$

C(46)-Fe(1)-C(47)-C(48)

$\mathrm{C}(41)-\mathrm{Fe}(1)-\mathrm{C}(47)-\mathrm{C}(48)$

$\mathrm{C}(44)-\mathrm{Fe}(1)-\mathrm{C}(47)-\mathrm{C}(46)$

$\mathrm{C}(45)-\mathrm{Fe}(1)-\mathrm{C}(47)-\mathrm{C}(46)$

$\mathrm{C}(43)-\mathrm{Fe}(1)-\mathrm{C}(47)-\mathrm{C}(46)$

$\mathrm{C}(40)-\mathrm{Fe}(1)-\mathrm{C}(47)-\mathrm{C}(46)$

C(39)-Fe(1)-C(47)-C(46)

$\mathrm{C}(42)-\mathrm{Fe}(1)-\mathrm{C}(47)-\mathrm{C}(46)$

$\mathrm{C}(48)-\mathrm{Fe}(1)-\mathrm{C}(47)-\mathrm{C}(46)$

$\mathrm{C}(41)-\mathrm{Fe}(1)-\mathrm{C}(47)-\mathrm{C}(46)$

C(46)-C(47)-C(48)-C(44)

$\mathrm{Fe}(1)-\mathrm{C}(47)-\mathrm{C}(48)-\mathrm{C}(44)$
$-119.48(18)$

43(3)

142.83(13)

$-179.36(13)$

61.93(19)

$-36.96(13)$

99.24(14)

$38.15(12)$

163(3)

$-97.68(13)$

$-59.87(18)$

$-178.58(14)$

$82.53(13)$

$-141.27(13)$

$119.48(18)$

$-0.2(3)$

$58.50(16)$

$-58.67(15)$

$-38.03(13)$

$-82.55(14)$

61.52(18)

$-179.95(13)$

13(9)

99.24(14)

$-120.28(19)$

142.64(13)

$82.25(14)$

37.73(13)

$-178.20(13)$

$-59.68(19)$

133(9)

$-140.49(13)$

120.28(19)

$-97.09(14)$

$0.0(3)$

$58.50(15)$ 


$\begin{array}{lc}\mathrm{C}(46)-\mathrm{C}(47)-\mathrm{C}(48)-\mathrm{Fe}(1) & -58.52(16) \\ \mathrm{C}(45)-\mathrm{C}(44)-\mathrm{C}(48)-\mathrm{C}(47) & 0.2(2) \\ \mathrm{P}(2)-\mathrm{C}(44)-\mathrm{C}(48)-\mathrm{C}(47) & -173.97(17) \\ \mathrm{Fe}(1)-\mathrm{C}(44)-\mathrm{C}(48)-\mathrm{C}(47) & -59.54(16) \\ \mathrm{C}(45)-\mathrm{C}(44)-\mathrm{C}(48)-\mathrm{Fe}(1) & 59.75(14) \\ \mathrm{P}(2)-\mathrm{C}(44)-\mathrm{C}(48)-\mathrm{Fe}(1) & -114.42(19) \\ \mathrm{C}(44)-\mathrm{Fe}(1)-\mathrm{C}(48)-\mathrm{C}(47) & 119.41(19) \\ \mathrm{C}(45)-\mathrm{Fe}(1)-\mathrm{C}(48)-\mathrm{C}(47) & 80.99(14) \\ \mathrm{C}(43)-\mathrm{Fe}(1)-\mathrm{C}(48)-\mathrm{C}(47) & -141.14(13) \\ \mathrm{C}(40)-\mathrm{Fe}(1)-\mathrm{C}(48)-\mathrm{C}(47) & 5(15) \\ \mathrm{C}(39)-\mathrm{Fe}(1)-\mathrm{C}(48)-\mathrm{C}(47) & -179.81(13) \\ \mathrm{C}(42)-\mathrm{Fe}(1)-\mathrm{C}(48)-\mathrm{C}(47) & -97.26(14) \\ \mathrm{C}(46)-\mathrm{Fe}(1)-\mathrm{C}(48)-\mathrm{C}(47) & 37.02(13) \\ \mathrm{C}(41)-\mathrm{Fe}(1)-\mathrm{C}(48)-\mathrm{C}(47) & -60.0(2) \\ \mathrm{C}(45)-\mathrm{Fe}(1)-\mathrm{C}(48)-\mathrm{C}(44) & -38.42(13) \\ \mathrm{C}(43)-\mathrm{Fe}(1)-\mathrm{C}(48)-\mathrm{C}(44) & 99.46(13) \\ \mathrm{C}(40)-\mathrm{Fe}(1)-\mathrm{C}(48)-\mathrm{C}(44) & -115(15) \\ \mathrm{C}(39)-\mathrm{Fe}(1)-\mathrm{C}(48)-\mathrm{C}(44) & 60.78(18) \\ \mathrm{C}(42)-\mathrm{Fe}(1)-\mathrm{C}(48)-\mathrm{C}(44) & 143.33(13) \\ \mathrm{C}(46)-\mathrm{Fe}(1)-\mathrm{C}(48)-\mathrm{C}(44) & -82.39(14) \\ \mathrm{C}(41)-\mathrm{Fe}(1)-\mathrm{C}(48)-\mathrm{C}(44) & -179.36(14) \\ \mathrm{C}(47)-\mathrm{Fe}(1)-\mathrm{C}(48)-\mathrm{C}(44) & -119.41(19) \\ & \end{array}$

Symmetry transformations used to generate equivalent atoms: 
Table S4g. Least-squares planes ( $\mathrm{x}, \mathrm{y}, \mathrm{z}$ in crystal coordinates) and deviations from them for $\mathbf{8 a}$ (* indicates atom used to define plane)

$-3.7741(0.0033) x+11.5798(0.0059) y+14.7320(0.0046) z=1.4413(0.0016)$

* $-0.0500(0.0011) \mathrm{O} 1$

* $\quad 0.0488(0.0011) \mathrm{C} 1$

* $-0.0177(0.0004)$ P1

* $0.0189(0.0004) \mathrm{P} 2$

$0.0726(0.0006) \mathrm{Ni1}$

Rms deviation of fitted atoms $=0.0373$ 
Table S5a. Crystal data and structure refinement for $\mathbf{8 b}$.

Identification code

Empirical formula

Formula weight

Temperature

Wavelength

Crystal system

Space group

Unit cell dimensions

Volume

Z

Density (calculated)

Absorption coefficient

$\mathrm{F}(000)$

Crystal size

Theta range for data collection

Index ranges

Reflections collected

Independent reflections

Completeness to theta $=27.46^{\circ}$

Absorption correction

Max. and min. transmission

Refinement method

Data / restraints / parameters

Goodness-of-fit on $\mathrm{F}^{2}$

Final $\mathrm{R}$ indices [I $>2 \operatorname{sigma}(\mathrm{I})]$

$\mathrm{R}$ indices (all data)

Largest diff. peak and hole $\mathbf{8 b}$

C46 H42 Fe Ni O P2

787.30

150(1) K

$0.71069 \AA$

Monoclinic

C $2 / c$

$\mathrm{a}=12.9031(5) \AA$

$\alpha=90^{\circ}$.

$\mathrm{b}=23.5457(9) \AA$

$\beta=99.891(2)^{\circ}$.

$\mathrm{c}=14.1418(4) \AA$ $\gamma=90^{\circ}$.

4232.6(3) $\AA^{3}$

4

$1.235 \mathrm{Mg} / \mathrm{m}^{3}$

$0.895 \mathrm{~mm}^{-1}$

1640

$0.33 \times 0.18 \times 0.10 \mathrm{~mm}^{3}$

2.26 to $27.46^{\circ}$.

$-16<=\mathrm{h}<=16,-30<=\mathrm{k}<=30,-18<=\mathrm{l}<=18$

9435

$4847[\mathrm{R}(\mathrm{int})=0.0295]$

$99.6 \%$

Multi-scan

0.9159 and 0.7567

Full-matrix least-squares on $\mathrm{F}^{2}$

4847 / 0 / 255

1.171

$\mathrm{R} 1=0.0850, \mathrm{wR} 2=0.2244$

$\mathrm{R} 1=0.1064, \mathrm{wR} 2=0.2350$

1.514 and -0.628 e. $\AA^{-3}$ 
Table S5b. Atomic coordinates (x $\left.10^{4}\right)$ and equivalent isotropic displacement parameters $\left(\AA^{2} \times 10^{3}\right)$ for $\mathbf{8 b}$. $U(e q)$ is defined as one third of the trace of the orthogonalized $U^{i j}$ tensor.

\begin{tabular}{|c|c|c|c|c|}
\hline & $\mathrm{x}$ & $\mathrm{y}$ & $\mathrm{z}$ & $\mathrm{U}(\mathrm{eq})$ \\
\hline $\mathrm{Fe}(1)$ & 0 & $-476(1)$ & 7500 & $30(1)$ \\
\hline $\mathrm{Ni}(1)$ & 0 & 1304(1) & 7500 & $26(1)$ \\
\hline $\mathrm{P}(1)$ & $1275(1)$ & $760(1)$ & $7201(1)$ & $28(1)$ \\
\hline $\mathrm{O}(1)$ & $527(5)$ & $2038(2)$ & $7462(4)$ & $61(2)$ \\
\hline$C(1)$ & $527(5)$ & $2038(2)$ & $7462(4)$ & $61(2)$ \\
\hline$C(2)$ & $975(7)$ & 2529(4) & $7592(7)$ & $23(2)$ \\
\hline$C(3)$ & $2156(8)$ & $2425(5)$ & $7687(8)$ & $31(2)$ \\
\hline$C(4)$ & $2837(11)$ & $2494(5)$ & $8729(8)$ & $41(3)$ \\
\hline$C(5)$ & $3874(14)$ & $2389(6)$ & $8822(12)$ & $69(5)$ \\
\hline$C(6)$ & $4447(15)$ & $2463(7)$ & $9806(10)$ & $72(6)$ \\
\hline$C(7)$ & $547(4)$ & $3163(3)$ & $7556(4)$ & $38(1)$ \\
\hline$C(8)$ & 1071(7) & $3678(4)$ & $7616(5)$ & $69(3)$ \\
\hline $\mathrm{C}(9)$ & $569(12)$ & 4173(4) & $7568(8)$ & $137(9)$ \\
\hline$C(10)$ & $2372(4)$ & $750(3)$ & $8202(4)$ & $34(1)$ \\
\hline $\mathrm{C}(11)$ & $3395(4)$ & $635(3)$ & $8110(4)$ & $42(2)$ \\
\hline$C(12)$ & $4204(5)$ & $663(4)$ & $8906(5)$ & $53(2)$ \\
\hline$C(13)$ & $3995(5)$ & 797(4) & $9792(5)$ & $54(2)$ \\
\hline$C(14)$ & $2956(5)$ & $906(3)$ & $9900(4)$ & $47(2)$ \\
\hline$C(15)$ & $2165(5)$ & $892(3)$ & 9114(4) & $37(1)$ \\
\hline$C(16)$ & 1874(4) & $964(3)$ & $6166(4)$ & $37(1)$ \\
\hline $\mathrm{C}(17)$ & $2415(5)$ & $571(3)$ & $5700(4)$ & $42(2)$ \\
\hline $\mathrm{C}(18)$ & $2898(5)$ & $736(4)$ & $4929(5)$ & $51(2)$ \\
\hline$C(19)$ & 2818(8) & $1282(4)$ & $4600(6)$ & $76(3)$ \\
\hline$C(20)$ & 2197(10) & 1673(4) & $5014(7)$ & $99(4)$ \\
\hline $\mathrm{C}(21)$ & 1776(8) & $1510(3)$ & $5818(6)$ & $66(2)$ \\
\hline $\mathrm{C}(22)$ & 1023(4) & $18(3)$ & 6932(4) & $31(1)$ \\
\hline $\mathrm{C}(23)$ & $182(4)$ & $-173(3)$ & 6192(4) & $34(1)$ \\
\hline $\mathrm{C}(24)$ & $204(5)$ & $-776(3)$ & $6180(5)$ & $46(2)$ \\
\hline $\mathrm{C}(25)$ & $1058(6)$ & $-960(3)$ & $6910(5)$ & $53(2)$ \\
\hline$C(26)$ & $1548(5)$ & $-474(3)$ & $7371(4)$ & $38(1)$ \\
\hline
\end{tabular}


Table S5c. Bond lengths $[\AA]$ and angles $\left[{ }^{\circ}\right]$ for $\mathbf{8 b}$.

\begin{tabular}{|c|c|}
\hline $\mathrm{Fe}(1)-\mathrm{C}(22)$ & $2.026(6)$ \\
\hline $\mathrm{Fe}(1)-\mathrm{C}(22) \# 1$ & $2.026(6)$ \\
\hline $\mathrm{Fe}(1)-\mathrm{C}(23) \# 1$ & $2.033(6)$ \\
\hline $\mathrm{Fe}(1)-\mathrm{C}(23)$ & $2.033(6)$ \\
\hline $\mathrm{Fe}(1)-\mathrm{C}(26) \# 1$ & $2.037(6)$ \\
\hline $\mathrm{Fe}(1)-\mathrm{C}(26)$ & $2.037(6)$ \\
\hline $\mathrm{Fe}(1)-\mathrm{C}(24)$ & $2.055(6)$ \\
\hline $\mathrm{Fe}(1)-\mathrm{C}(24) \# 1$ & $2.055(6)$ \\
\hline $\mathrm{Fe}(1)-\mathrm{C}(25)$ & $2.061(7)$ \\
\hline $\mathrm{Fe}(1)-\mathrm{C}(25) \# 1$ & $2.061(7)$ \\
\hline $\mathrm{Ni}(1)-\mathrm{O}(1)$ & $1.862(5)$ \\
\hline $\mathrm{Ni}(1)-\mathrm{C}(1) \# 1$ & $1.862(5)$ \\
\hline $\mathrm{Ni}(1)-\mathrm{O}(1) \# 1$ & $1.862(5)$ \\
\hline Ni(1)-P(1) & $2.1832(15)$ \\
\hline $\mathrm{Ni}(1)-\mathrm{P}(1) \# 1$ & $2.1833(15)$ \\
\hline $\mathrm{P}(1)-\mathrm{C}(22)$ & $1.804(6)$ \\
\hline $\mathrm{P}(1)-\mathrm{C}(10)$ & $1.823(6)$ \\
\hline$P(1)-C(16)$ & $1.832(6)$ \\
\hline $\mathrm{O}(1)-\mathrm{C}(2)$ & $1.291(11)$ \\
\hline $\mathrm{O}(1)-\mathrm{C}(1) \# 1$ & $1.383(14)$ \\
\hline $\mathrm{O}(1)-\mathrm{O}(1) \# 1$ & $1.383(14)$ \\
\hline $\mathrm{C}(2)-\mathrm{C}(3)$ & $1.526(13)$ \\
\hline $\mathrm{C}(3)-\mathrm{C}(4)$ & $1.589(16)$ \\
\hline $\mathrm{C}(3)-\mathrm{H}(3 \mathrm{~A})$ & 0.9900 \\
\hline $\mathrm{C}(3)-\mathrm{H}(3 \mathrm{~B})$ & 0.9900 \\
\hline$C(4)-C(5)$ & $1.34(2)$ \\
\hline $\mathrm{C}(4)-\mathrm{H}(4 \mathrm{~A})$ & 0.9900 \\
\hline $\mathrm{C}(4)-\mathrm{H}(4 \mathrm{~B})$ & 0.9900 \\
\hline$C(5)-C(6)$ & $1.470(19)$ \\
\hline $\mathrm{C}(5)-\mathrm{H}(5 \mathrm{~A})$ & 0.9900 \\
\hline $\mathrm{C}(5)-\mathrm{H}(5 \mathrm{~B})$ & 0.9900 \\
\hline $\mathrm{C}(6)-\mathrm{H}(6 \mathrm{~A})$ & 0.9800 \\
\hline $\mathrm{C}(6)-\mathrm{H}(6 \mathrm{~B})$ & 0.9800 \\
\hline $\mathrm{C}(6)-\mathrm{H}(6 \mathrm{C})$ & 0.9800 \\
\hline
\end{tabular}




\begin{tabular}{|c|c|}
\hline $\mathrm{C}(7)-\mathrm{C}(8)$ & $1.385(10)$ \\
\hline$C(7)-C(7) \# 1$ & $1.393(11)$ \\
\hline $\mathrm{C}(7)-\mathrm{H}(7)$ & 0.9500 \\
\hline $\mathrm{C}(8)-\mathrm{C}(9)$ & $1.330(15)$ \\
\hline $\mathrm{C}(8)-\mathrm{H}(8)$ & 0.9500 \\
\hline $\mathrm{C}(9)-\mathrm{C}(9) \# 1$ & $1.45(3)$ \\
\hline $\mathrm{C}(9)-\mathrm{H}(9)$ & 0.9500 \\
\hline$C(10)-C(11)$ & $1.375(8)$ \\
\hline$C(10)-C(15)$ & $1.402(8)$ \\
\hline$C(11)-C(12)$ & $1.399(9)$ \\
\hline $\mathrm{C}(11)-\mathrm{H}(11)$ & 0.9500 \\
\hline$C(12)-C(13)$ & $1.362(10)$ \\
\hline $\mathrm{C}(12)-\mathrm{H}(12)$ & 0.9500 \\
\hline$C(13)-C(14)$ & $1.398(10)$ \\
\hline $\mathrm{C}(13)-\mathrm{H}(13)$ & 0.9500 \\
\hline$C(14)-C(15)$ & $1.374(8)$ \\
\hline $\mathrm{C}(14)-\mathrm{H}(14)$ & 0.9500 \\
\hline $\mathrm{C}(15)-\mathrm{H}(15)$ & 0.9500 \\
\hline$C(16)-C(21)$ & $1.375(10)$ \\
\hline$C(16)-C(17)$ & $1.391(9)$ \\
\hline $\mathrm{C}(17)-\mathrm{C}(18)$ & $1.401(9)$ \\
\hline $\mathrm{C}(17)-\mathrm{H}(17)$ & 0.9500 \\
\hline $\mathrm{C}(18)-\mathrm{C}(19)$ & $1.365(12)$ \\
\hline $\mathrm{C}(18)-\mathrm{H}(18)$ & 0.9500 \\
\hline $\mathrm{C}(19)-\mathrm{C}(20)$ & $1.412(13)$ \\
\hline $\mathrm{C}(19)-\mathrm{H}(19)$ & 0.9500 \\
\hline $\mathrm{C}(20)-\mathrm{C}(21)$ & $1.396(10)$ \\
\hline $\mathrm{C}(20)-\mathrm{H}(20)$ & 0.9500 \\
\hline $\mathrm{C}(21)-\mathrm{H}(21)$ & 0.9500 \\
\hline$C(22)-C(26)$ & $1.429(8)$ \\
\hline$C(22)-C(23)$ & $1.444(7)$ \\
\hline$C(23)-C(24)$ & $1.420(9)$ \\
\hline $\mathrm{C}(23)-\mathrm{H}(23)$ & 0.9500 \\
\hline$C(24)-C(25)$ & $1.442(10)$ \\
\hline $\mathrm{C}(24)-\mathrm{H}(24)$ & 0.9500 \\
\hline$C(25)-C(26)$ & $1.411(9)$ \\
\hline
\end{tabular}




\begin{tabular}{|c|c|}
\hline $\mathrm{C}(25)-\mathrm{H}(25)$ & 0.9500 \\
\hline $\mathrm{C}(26)-\mathrm{H}(26)$ & 0.9500 \\
\hline $\mathrm{C}(22)-\mathrm{Fe}(1)-\mathrm{C}(22) \# 1$ & $109.9(3)$ \\
\hline$C(22)-\mathrm{Fe}(1)-\mathrm{C}(23) \# 1$ & $110.1(2)$ \\
\hline $\mathrm{C}(22) \# 1-\mathrm{Fe}(1)-\mathrm{C}(23) \# 1$ & $41.7(2)$ \\
\hline $\mathrm{C}(22)-\mathrm{Fe}(1)-\mathrm{C}(23)$ & $41.7(2)$ \\
\hline $\mathrm{C}(22) \# 1-\mathrm{Fe}(1)-\mathrm{C}(23)$ & $110.1(2)$ \\
\hline $\mathrm{C}(23) \# 1-\mathrm{Fe}(1)-\mathrm{C}(23)$ & $138.9(3)$ \\
\hline$C(22)-\mathrm{Fe}(1)-\mathrm{C}(26) \# 1$ & $138.5(2)$ \\
\hline $\mathrm{C}(22) \# 1-\mathrm{Fe}(1)-\mathrm{C}(26) \# 1$ & $41.2(2)$ \\
\hline $\mathrm{C}(23) \# 1-\mathrm{Fe}(1)-\mathrm{C}(26) \# 1$ & $69.5(2)$ \\
\hline$C(23)-\mathrm{Fe}(1)-\mathrm{C}(26) \# 1$ & $110.4(2)$ \\
\hline $\mathrm{C}(22)-\mathrm{Fe}(1)-\mathrm{C}(26)$ & $41.2(2)$ \\
\hline $\mathrm{C}(22) \# 1-\mathrm{Fe}(1)-\mathrm{C}(26)$ & $138.5(2)$ \\
\hline $\mathrm{C}(23) \# 1-\mathrm{Fe}(1)-\mathrm{C}(26)$ & $110.4(2)$ \\
\hline $\mathrm{C}(23)-\mathrm{Fe}(1)-\mathrm{C}(26)$ & $69.5(2)$ \\
\hline $\mathrm{C}(26) \# 1-\mathrm{Fe}(1)-\mathrm{C}(26)$ & $179.7(4)$ \\
\hline $\mathrm{C}(22)-\mathrm{Fe}(1)-\mathrm{C}(24)$ & $69.1(2)$ \\
\hline $\mathrm{C}(22) \# 1-\mathrm{Fe}(1)-\mathrm{C}(24)$ & $138.7(2)$ \\
\hline $\mathrm{C}(23) \# 1-\mathrm{Fe}(1)-\mathrm{C}(24)$ & $179.2(3)$ \\
\hline $\mathrm{C}(23)-\mathrm{Fe}(1)-\mathrm{C}(24)$ & $40.7(3)$ \\
\hline $\mathrm{C}(26) \# 1-\mathrm{Fe}(1)-\mathrm{C}(24)$ & $111.3(3)$ \\
\hline $\mathrm{C}(26)-\mathrm{Fe}(1)-\mathrm{C}(24)$ & $68.9(3)$ \\
\hline $\mathrm{C}(22)-\mathrm{Fe}(1)-\mathrm{C}(24) \# 1$ & $138.7(2)$ \\
\hline $\mathrm{C}(22) \# 1-\mathrm{Fe}(1)-\mathrm{C}(24) \# 1$ & $69.1(2)$ \\
\hline $\mathrm{C}(23) \# 1-\mathrm{Fe}(1)-\mathrm{C}(24) \# 1$ & $40.7(3)$ \\
\hline $\mathrm{C}(23)-\mathrm{Fe}(1)-\mathrm{C}(24) \# 1$ & $179.2(3)$ \\
\hline $\mathrm{C}(26) \# 1-\mathrm{Fe}(1)-\mathrm{C}(24) \# 1$ & $68.9(3)$ \\
\hline $\mathrm{C}(26)-\mathrm{Fe}(1)-\mathrm{C}(24) \# 1$ & $111.3(3)$ \\
\hline $\mathrm{C}(24)-\mathrm{Fe}(1)-\mathrm{C}(24) \# 1$ & $139.8(4)$ \\
\hline $\mathrm{C}(22)-\mathrm{Fe}(1)-\mathrm{C}(25)$ & $68.6(3)$ \\
\hline $\mathrm{C}(22) \# 1-\mathrm{Fe}(1)-\mathrm{C}(25)$ & $178.5(3)$ \\
\hline $\mathrm{C}(23) \# 1-\mathrm{Fe}(1)-\mathrm{C}(25)$ & $138.6(3)$ \\
\hline $\mathrm{C}(23)-\mathrm{Fe}(1)-\mathrm{C}(25)$ & $68.8(3)$ \\
\hline $\mathrm{C}(26) \# 1-\mathrm{Fe}(1)-\mathrm{C}(25)$ & $140.0(3)$ \\
\hline
\end{tabular}




\begin{tabular}{|c|c|}
\hline $\mathrm{C}(26)-\mathrm{Fe}(1)-\mathrm{C}(25)$ & $40.3(3)$ \\
\hline $\mathrm{C}(24)-\mathrm{Fe}(1)-\mathrm{C}(25)$ & $41.0(3)$ \\
\hline $\mathrm{C}(24) \# 1-\mathrm{Fe}(1)-\mathrm{C}(25)$ & $112.0(3)$ \\
\hline $\mathrm{C}(22)-\mathrm{Fe}(1)-\mathrm{C}(25) \# 1$ & $178.5(3)$ \\
\hline $\mathrm{C}(22) \# 1-\mathrm{Fe}(1)-\mathrm{C}(25) \# 1$ & $68.6(3)$ \\
\hline $\mathrm{C}(23) \# 1-\mathrm{Fe}(1)-\mathrm{C}(25) \# 1$ & $68.8(3)$ \\
\hline $\mathrm{C}(23)-\mathrm{Fe}(1)-\mathrm{C}(25) \# 1$ & $138.6(3)$ \\
\hline $\mathrm{C}(26) \# 1-\mathrm{Fe}(1)-\mathrm{C}(25) \# 1$ & $40.3(3)$ \\
\hline $\mathrm{C}(26)-\mathrm{Fe}(1)-\mathrm{C}(25) \# 1$ & $140.0(3)$ \\
\hline $\mathrm{C}(24)-\mathrm{Fe}(1)-\mathrm{C}(25) \# 1$ & $112.0(3)$ \\
\hline $\mathrm{C}(24) \# 1-\mathrm{Fe}(1)-\mathrm{C}(25) \# 1$ & $41.0(3)$ \\
\hline $\mathrm{C}(25)-\mathrm{Fe}(1)-\mathrm{C}(25) \# 1$ & $112.9(4)$ \\
\hline $\mathrm{O}(1)-\mathrm{Ni}(1)-\mathrm{C}(1) \# 1$ & $43.6(4)$ \\
\hline $\mathrm{O}(1)-\mathrm{Ni}(1)-\mathrm{O}(1) \# 1$ & $43.6(4)$ \\
\hline $\mathrm{C}(1) \# 1-\mathrm{Ni}(1)-\mathrm{O}(1) \# 1$ & $0.0(6)$ \\
\hline $\mathrm{O}(1)-\mathrm{Ni}(1)-\mathrm{P}(1)$ & $104.4(2)$ \\
\hline $\mathrm{C}(1) \# 1-\mathrm{Ni}(1)-\mathrm{P}(1)$ & $147.3(2)$ \\
\hline $\mathrm{O}(1) \# 1-\mathrm{Ni}(1)-\mathrm{P}(1)$ & $147.3(2)$ \\
\hline $\mathrm{O}(1)-\mathrm{Ni}(1)-\mathrm{P}(1) \# 1$ & $147.3(2)$ \\
\hline $\mathrm{C}(1) \# 1-\mathrm{Ni}(1)-\mathrm{P}(1) \# 1$ & $104.4(2)$ \\
\hline $\mathrm{O}(1) \# 1-\mathrm{Ni}(1)-\mathrm{P}(1) \# 1$ & $104.4(2)$ \\
\hline $\mathrm{P}(1)-\mathrm{Ni}(1)-\mathrm{P}(1) \# 1$ & $108.13(8)$ \\
\hline$C(22)-P(1)-C(10)$ & $103.8(3)$ \\
\hline $\mathrm{C}(22)-\mathrm{P}(1)-\mathrm{C}(16)$ & $99.9(3)$ \\
\hline$C(10)-P(1)-C(16)$ & 104.2(3) \\
\hline $\mathrm{C}(22)-\mathrm{P}(1)-\mathrm{Ni}(1)$ & $119.60(18)$ \\
\hline $\mathrm{C}(10)-\mathrm{P}(1)-\mathrm{Ni}(1)$ & $111.56(19)$ \\
\hline $\mathrm{C}(16)-\mathrm{P}(1)-\mathrm{Ni}(1)$ & $115.8(2)$ \\
\hline $\mathrm{C}(2)-\mathrm{O}(1)-\mathrm{C}(1) \# 1$ & $114.3(6)$ \\
\hline $\mathrm{C}(2)-\mathrm{O}(1)-\mathrm{O}(1) \# 1$ & $114.3(6)$ \\
\hline $\mathrm{C}(1) \# 1-\mathrm{O}(1)-\mathrm{O}(1) \# 1$ & $0.0(5)$ \\
\hline $\mathrm{C}(2)-\mathrm{O}(1)-\mathrm{Ni}(1)$ & $169.7(6)$ \\
\hline $\mathrm{C}(1) \# 1-\mathrm{O}(1)-\mathrm{Ni}(1)$ & $68.2(2)$ \\
\hline $\mathrm{O}(1) \# 1-\mathrm{O}(1)-\mathrm{Ni}(1)$ & $68.2(2)$ \\
\hline $\mathrm{O}(1)-\mathrm{C}(2)-\mathrm{C}(3)$ & 106.6(8) \\
\hline$C(2)-C(3)-C(4)$ & $116.7(9)$ \\
\hline
\end{tabular}




\begin{tabular}{|c|c|}
\hline $\mathrm{C}(2)-\mathrm{C}(3)-\mathrm{H}(3 \mathrm{~A})$ & 108.1 \\
\hline $\mathrm{C}(4)-\mathrm{C}(3)-\mathrm{H}(3 \mathrm{~A})$ & 108.1 \\
\hline $\mathrm{C}(2)-\mathrm{C}(3)-\mathrm{H}(3 \mathrm{~B})$ & 108.1 \\
\hline $\mathrm{C}(4)-\mathrm{C}(3)-\mathrm{H}(3 \mathrm{~B})$ & 108.1 \\
\hline $\mathrm{H}(3 \mathrm{~A})-\mathrm{C}(3)-\mathrm{H}(3 \mathrm{~B})$ & 107.3 \\
\hline$C(5)-C(4)-C(3)$ & $117.0(12)$ \\
\hline $\mathrm{C}(5)-\mathrm{C}(4)-\mathrm{H}(4 \mathrm{~A})$ & 108.0 \\
\hline $\mathrm{C}(3)-\mathrm{C}(4)-\mathrm{H}(4 \mathrm{~A})$ & 108.0 \\
\hline $\mathrm{C}(5)-\mathrm{C}(4)-\mathrm{H}(4 \mathrm{~B})$ & 108.0 \\
\hline $\mathrm{C}(3)-\mathrm{C}(4)-\mathrm{H}(4 \mathrm{~B})$ & 108.0 \\
\hline $\mathrm{H}(4 \mathrm{~A})-\mathrm{C}(4)-\mathrm{H}(4 \mathrm{~B})$ & 107.3 \\
\hline$C(4)-C(5)-C(6)$ & $113.7(18)$ \\
\hline $\mathrm{C}(4)-\mathrm{C}(5)-\mathrm{H}(5 \mathrm{~A})$ & 108.8 \\
\hline $\mathrm{C}(6)-\mathrm{C}(5)-\mathrm{H}(5 \mathrm{~A})$ & 108.8 \\
\hline $\mathrm{C}(4)-\mathrm{C}(5)-\mathrm{H}(5 \mathrm{~B})$ & 108.8 \\
\hline $\mathrm{C}(6)-\mathrm{C}(5)-\mathrm{H}(5 \mathrm{~B})$ & 108.8 \\
\hline $\mathrm{H}(5 \mathrm{~A})-\mathrm{C}(5)-\mathrm{H}(5 \mathrm{~B})$ & 107.7 \\
\hline $\mathrm{C}(5)-\mathrm{C}(6)-\mathrm{H}(6 \mathrm{~A})$ & 109.5 \\
\hline $\mathrm{C}(5)-\mathrm{C}(6)-\mathrm{H}(6 \mathrm{~B})$ & 109.5 \\
\hline $\mathrm{H}(6 \mathrm{~A})-\mathrm{C}(6)-\mathrm{H}(6 \mathrm{~B})$ & 109.5 \\
\hline $\mathrm{C}(5)-\mathrm{C}(6)-\mathrm{H}(6 \mathrm{C})$ & 109.5 \\
\hline $\mathrm{H}(6 \mathrm{~A})-\mathrm{C}(6)-\mathrm{H}(6 \mathrm{C})$ & 109.5 \\
\hline $\mathrm{H}(6 \mathrm{~B})-\mathrm{C}(6)-\mathrm{H}(6 \mathrm{C})$ & 109.5 \\
\hline$C(8)-C(7)-C(7) \# 1$ & $118.8(5)$ \\
\hline $\mathrm{C}(8)-\mathrm{C}(7)-\mathrm{H}(7)$ & 120.6 \\
\hline $\mathrm{C}(7) \# 1-\mathrm{C}(7)-\mathrm{H}(7)$ & 120.6 \\
\hline $\mathrm{C}(9)-\mathrm{C}(8)-\mathrm{C}(7)$ & $122.5(9)$ \\
\hline $\mathrm{C}(9)-\mathrm{C}(8)-\mathrm{H}(8)$ & 118.7 \\
\hline $\mathrm{C}(7)-\mathrm{C}(8)-\mathrm{H}(8)$ & 118.7 \\
\hline $\mathrm{C}(8)-\mathrm{C}(9)-\mathrm{C}(9) \# 1$ & $118.7(6)$ \\
\hline $\mathrm{C}(8)-\mathrm{C}(9)-\mathrm{H}(9)$ & 120.6 \\
\hline $\mathrm{C}(9) \# 1-\mathrm{C}(9)-\mathrm{H}(9)$ & 120.6 \\
\hline $\mathrm{C}(11)-\mathrm{C}(10)-\mathrm{C}(15)$ & $118.2(5)$ \\
\hline $\mathrm{C}(11)-\mathrm{C}(10)-\mathrm{P}(1)$ & $124.0(4)$ \\
\hline $\mathrm{C}(15)-\mathrm{C}(10)-\mathrm{P}(1)$ & $117.8(4)$ \\
\hline $\mathrm{C}(10)-\mathrm{C}(11)-\mathrm{C}(12)$ & $120.6(6)$ \\
\hline
\end{tabular}




\begin{tabular}{|c|c|}
\hline $\mathrm{C}(10)-\mathrm{C}(11)-\mathrm{H}(11)$ & 119.7 \\
\hline $\mathrm{C}(12)-\mathrm{C}(11)-\mathrm{H}(11)$ & 119.7 \\
\hline$C(13)-C(12)-C(11)$ & $120.9(6)$ \\
\hline $\mathrm{C}(13)-\mathrm{C}(12)-\mathrm{H}(12)$ & 119.6 \\
\hline $\mathrm{C}(11)-\mathrm{C}(12)-\mathrm{H}(12)$ & 119.6 \\
\hline $\mathrm{C}(12)-\mathrm{C}(13)-\mathrm{C}(14)$ & $119.2(6)$ \\
\hline $\mathrm{C}(12)-\mathrm{C}(13)-\mathrm{H}(13)$ & 120.4 \\
\hline $\mathrm{C}(14)-\mathrm{C}(13)-\mathrm{H}(13)$ & 120.4 \\
\hline $\mathrm{C}(15)-\mathrm{C}(14)-\mathrm{C}(13)$ & $120.0(6)$ \\
\hline $\mathrm{C}(15)-\mathrm{C}(14)-\mathrm{H}(14)$ & 120.0 \\
\hline $\mathrm{C}(13)-\mathrm{C}(14)-\mathrm{H}(14)$ & 120.0 \\
\hline $\mathrm{C}(14)-\mathrm{C}(15)-\mathrm{C}(10)$ & 121.1(6) \\
\hline $\mathrm{C}(14)-\mathrm{C}(15)-\mathrm{H}(15)$ & 119.4 \\
\hline $\mathrm{C}(10)-\mathrm{C}(15)-\mathrm{H}(15)$ & 119.4 \\
\hline $\mathrm{C}(21)-\mathrm{C}(16)-\mathrm{C}(17)$ & $118.4(6)$ \\
\hline $\mathrm{C}(21)-\mathrm{C}(16)-\mathrm{P}(1)$ & $120.4(5)$ \\
\hline $\mathrm{C}(17)-\mathrm{C}(16)-\mathrm{P}(1)$ & $121.2(5)$ \\
\hline $\mathrm{C}(16)-\mathrm{C}(17)-\mathrm{C}(18)$ & $120.8(7)$ \\
\hline $\mathrm{C}(16)-\mathrm{C}(17)-\mathrm{H}(17)$ & 119.6 \\
\hline $\mathrm{C}(18)-\mathrm{C}(17)-\mathrm{H}(17)$ & 119.6 \\
\hline $\mathrm{C}(19)-\mathrm{C}(18)-\mathrm{C}(17)$ & $120.6(7)$ \\
\hline $\mathrm{C}(19)-\mathrm{C}(18)-\mathrm{H}(18)$ & 119.7 \\
\hline $\mathrm{C}(17)-\mathrm{C}(18)-\mathrm{H}(18)$ & 119.7 \\
\hline $\mathrm{C}(18)-\mathrm{C}(19)-\mathrm{C}(20)$ & $119.2(7)$ \\
\hline $\mathrm{C}(18)-\mathrm{C}(19)-\mathrm{H}(19)$ & 120.4 \\
\hline $\mathrm{C}(20)-\mathrm{C}(19)-\mathrm{H}(19)$ & 120.4 \\
\hline $\mathrm{C}(21)-\mathrm{C}(20)-\mathrm{C}(19)$ & 119.1(9) \\
\hline $\mathrm{C}(21)-\mathrm{C}(20)-\mathrm{H}(20)$ & 120.4 \\
\hline $\mathrm{C}(19)-\mathrm{C}(20)-\mathrm{H}(20)$ & 120.4 \\
\hline$C(16)-C(21)-C(20)$ & $121.6(8)$ \\
\hline $\mathrm{C}(16)-\mathrm{C}(21)-\mathrm{H}(21)$ & 119.2 \\
\hline $\mathrm{C}(20)-\mathrm{C}(21)-\mathrm{H}(21)$ & 119.2 \\
\hline $\mathrm{C}(26)-\mathrm{C}(22)-\mathrm{C}(23)$ & $107.7(5)$ \\
\hline $\mathrm{C}(26)-\mathrm{C}(22)-\mathrm{P}(1)$ & $129.6(4)$ \\
\hline $\mathrm{C}(23)-\mathrm{C}(22)-\mathrm{P}(1)$ & $122.7(4)$ \\
\hline $\mathrm{C}(26)-\mathrm{C}(22)-\mathrm{Fe}(1)$ & $69.8(3)$ \\
\hline
\end{tabular}




$\begin{array}{lc}\mathrm{C}(23)-\mathrm{C}(22)-\mathrm{Fe}(1) & 69.4(3) \\ \mathrm{P}(1)-\mathrm{C}(22)-\mathrm{Fe}(1) & 125.4(3) \\ \mathrm{C}(24)-\mathrm{C}(23)-\mathrm{C}(22) & 107.9(5) \\ \mathrm{C}(24)-\mathrm{C}(23)-\mathrm{Fe}(1) & 70.5(4) \\ \mathrm{C}(22)-\mathrm{C}(23)-\mathrm{Fe}(1) & 68.9(3) \\ \mathrm{C}(24)-\mathrm{C}(23)-\mathrm{H}(23) & 126.1 \\ \mathrm{C}(22)-\mathrm{C}(23)-\mathrm{H}(23) & 126.1 \\ \mathrm{Fe}(1)-\mathrm{C}(23)-\mathrm{H}(23) & 126.1 \\ \mathrm{C}(23)-\mathrm{C}(24)-\mathrm{C}(25) & 107.8(6) \\ \mathrm{C}(23)-\mathrm{C}(24)-\mathrm{Fe}(1) & 68.9(3) \\ \mathrm{C}(25)-\mathrm{C}(24)-\mathrm{Fe}(1) & 69.7(4) \\ \mathrm{C}(23)-\mathrm{C}(24)-\mathrm{H}(24) & 126.1 \\ \mathrm{C}(25)-\mathrm{C}(24)-\mathrm{H}(24) & 126.1 \\ \mathrm{Fe}(1)-\mathrm{C}(24)-\mathrm{H}(24) & 126.9 \\ \mathrm{C}(26)-\mathrm{C}(25)-\mathrm{C}(24) & 108.4(6) \\ \mathrm{C}(26)-\mathrm{C}(25)-\mathrm{Fe}(1) & 68.9(4) \\ \mathrm{C}(24)-\mathrm{C}(25)-\mathrm{Fe}(1) & 69.3(4) \\ \mathrm{C}(26)-\mathrm{C}(25)-\mathrm{H}(25) & 125.8 \\ \mathrm{C}(24)-\mathrm{C}(25)-\mathrm{H}(25) & 125.8 \\ \mathrm{Fe}(1)-\mathrm{C}(25)-\mathrm{H}(25) & 127.6 \\ \mathrm{C}(25)-\mathrm{C}(26)-\mathrm{C}(22) & 108.4(6) \\ \mathrm{C}(25)-\mathrm{C}(26)-\mathrm{Fe}(1) & 70.8(4) \\ \mathrm{C}(22)-\mathrm{C}(26)-\mathrm{Fe}(1) & 69.0(3) \\ \mathrm{C}(25)-\mathrm{C}(26)-\mathrm{H}(26) & 125.8 \\ \mathrm{C}(22)-\mathrm{C}(26)-\mathrm{H}(26) & 125.8 \\ \mathrm{Fe}(1)-\mathrm{C}(26)-\mathrm{H}(26) & 126.0 \\ & \\ & \\ & \\ & \end{array}$

Symmetry transformations used to generate equivalent atoms:

\#1 -x,y,-z+3/2 
Table S5d. Anisotropic displacement parameters $\left(\AA^{2} \times 10^{3}\right)$ for $\mathbf{8 b}$. The anisotropic displacement factor exponent takes the form: $-2 \pi^{2}\left[h^{2} a^{* 2} U^{11}+\ldots+2 h k a^{*} b^{*} U^{12}\right]$

\begin{tabular}{|c|c|c|c|c|c|c|}
\hline & $\mathrm{U}^{11}$ & $\mathrm{U}^{22}$ & $\mathrm{U}^{33}$ & $\mathrm{U}^{23}$ & $\mathrm{U}^{13}$ & $\mathrm{U}^{12}$ \\
\hline $\mathrm{Fe}(1)$ & $31(1)$ & $26(1)$ & $30(1)$ & 0 & $1(1)$ & 0 \\
\hline $\mathrm{Ni}(1)$ & 21(1) & $25(1)$ & $31(1)$ & 0 & $4(1)$ & 0 \\
\hline $\mathrm{P}(1)$ & $22(1)$ & $37(1)$ & $24(1)$ & $-2(1)$ & $4(1)$ & $-2(1)$ \\
\hline $\mathrm{O}(1)$ & $79(4)$ & $34(3)$ & $56(3)$ & 11(3) & $-28(3)$ & $-20(3)$ \\
\hline $\mathrm{C}(1)$ & $79(4)$ & $34(3)$ & $56(3)$ & $11(3)$ & $-28(3)$ & $-20(3)$ \\
\hline$C(2)$ & $18(4)$ & $24(5)$ & $28(5)$ & $-4(4)$ & $1(4)$ & $1(4)$ \\
\hline$C(3)$ & $26(5)$ & $34(6)$ & $34(6)$ & $-9(5)$ & $5(4)$ & $1(4)$ \\
\hline$C(4)$ & $65(9)$ & $29(6)$ & $30(6)$ & $9(5)$ & $11(5)$ & $7(6)$ \\
\hline$C(5)$ & $81(12)$ & $40(8)$ & $70(10)$ & $24(7)$ & $-31(9)$ & $-31(8)$ \\
\hline$C(6)$ & $99(13)$ & $72(11)$ & $38(7)$ & $-2(7)$ & $-14(8)$ & $-61(10)$ \\
\hline$C(7)$ & $26(3)$ & $53(4)$ & $34(3)$ & $-9(3)$ & $7(2)$ & $-4(3)$ \\
\hline$C(8)$ & $76(5)$ & $99(7)$ & $38(4)$ & $-24(4)$ & $23(4)$ & $-63(5)$ \\
\hline$C(9)$ & $340(30)$ & $44(4)$ & $38(5)$ & $-16(5)$ & $49(12)$ & $-89(8)$ \\
\hline$C(10)$ & $32(3)$ & $41(3)$ & $28(3)$ & $-5(2)$ & $3(2)$ & $0(2)$ \\
\hline $\mathrm{C}(11)$ & $25(3)$ & $63(4)$ & $36(3)$ & $-14(3)$ & $3(2)$ & $2(3)$ \\
\hline$C(12)$ & $29(3)$ & $80(5)$ & $47(4)$ & $-8(4)$ & $-3(3)$ & $5(3)$ \\
\hline$C(13)$ & $41(4)$ & $77(5)$ & $38(3)$ & $-10(3)$ & $-12(3)$ & $-2(3)$ \\
\hline$C(14)$ & $44(3)$ & $65(4)$ & $28(3)$ & $-9(3)$ & $-1(3)$ & $-2(3)$ \\
\hline$C(15)$ & $31(3)$ & $44(3)$ & $34(3)$ & $-5(2)$ & $4(2)$ & $2(2)$ \\
\hline$C(16)$ & $31(3)$ & $52(4)$ & $28(3)$ & $-9(3)$ & $8(2)$ & $-14(3)$ \\
\hline $\mathrm{C}(17)$ & $28(3)$ & $70(4)$ & 27(3) & $0(3)$ & $4(2)$ & $0(3)$ \\
\hline$C(18)$ & $35(3)$ & $84(6)$ & $34(3)$ & $-12(3)$ & $9(3)$ & $-11(3)$ \\
\hline$C(19)$ & 113(7) & $74(6)$ & $58(5)$ & $-23(4)$ & $60(5)$ & $-50(5)$ \\
\hline$C(20)$ & $184(12)$ & $52(5)$ & $83(7)$ & $-18(5)$ & $86(8)$ & $-52(6)$ \\
\hline $\mathrm{C}(21)$ & 107(7) & 43(4) & $61(5)$ & $-16(4)$ & $51(5)$ & $-26(4)$ \\
\hline $\mathrm{C}(22)$ & $25(3)$ & $43(3)$ & $25(3)$ & $-3(2)$ & $5(2)$ & $1(2)$ \\
\hline$C(23)$ & $32(3)$ & $45(3)$ & $26(3)$ & $-4(2)$ & $3(2)$ & $-2(2)$ \\
\hline$C(24)$ & $48(4)$ & $47(4)$ & $41(3)$ & $-17(3)$ & $7(3)$ & $0(3)$ \\
\hline$C(25)$ & 61(4) & $38(4)$ & $57(4)$ & $-11(3)$ & $7(3)$ & $12(3)$ \\
\hline$C(26)$ & $33(3)$ & $42(3)$ & $38(3)$ & $-6(3)$ & $3(2)$ & $8(2)$ \\
\hline
\end{tabular}


Table S5e. Hydrogen coordinates $\left(\mathrm{x} 10^{4}\right)$ and isotropic displacement parameters $\left(\AA^{2} \times 10^{3}\right)$ for $\mathbf{8 b}$.

\begin{tabular}{|c|c|c|c|c|}
\hline & $\mathrm{x}$ & $\mathrm{y}$ & z & $\mathrm{U}(\mathrm{eq})$ \\
\hline $\mathrm{H}(3 \mathrm{~A})$ & 2437 & 2692 & 7251 & 38 \\
\hline $\mathrm{H}(3 \mathrm{~B})$ & 2263 & 2036 & 7458 & 38 \\
\hline $\mathrm{H}(4 \mathrm{~A})$ & 2746 & 2887 & 8949 & 49 \\
\hline $\mathrm{H}(4 \mathrm{~B})$ & 2542 & 2236 & 9168 & 49 \\
\hline $\mathrm{H}(5 \mathrm{~A})$ & 4180 & 2647 & 8390 & 83 \\
\hline $\mathrm{H}(5 \mathrm{~B})$ & 3975 & 1995 & 8613 & 83 \\
\hline $\mathrm{H}(6 \mathrm{~A})$ & 5193 & 2376 & 9825 & 109 \\
\hline $\mathrm{H}(6 \mathrm{~B})$ & 4156 & 2206 & 10238 & 109 \\
\hline $\mathrm{H}(6 \mathrm{C})$ & 4374 & 2856 & 10010 & 109 \\
\hline $\mathrm{H}(7)$ & 927 & 2815 & 7590 & 45 \\
\hline $\mathrm{H}(8)$ & 1817 & 3678 & 7694 & 83 \\
\hline $\mathrm{H}(9)$ & 950 & 4521 & 7622 & 164 \\
\hline $\mathrm{H}(11)$ & 3554 & 536 & 7499 & 50 \\
\hline $\mathrm{H}(12)$ & 4907 & 587 & 8829 & 64 \\
\hline $\mathrm{H}(13)$ & 4548 & 816 & 10329 & 65 \\
\hline $\mathrm{H}(14)$ & 2798 & 990 & 10516 & 56 \\
\hline $\mathrm{H}(15)$ & 1466 & 980 & 9190 & 44 \\
\hline $\mathrm{H}(17)$ & 2456 & 187 & 5907 & 50 \\
\hline $\mathrm{H}(18)$ & 3285 & 466 & 4632 & 61 \\
\hline $\mathrm{H}(19)$ & 3176 & 1398 & 4099 & 91 \\
\hline $\mathrm{H}(20)$ & 2068 & 2042 & 4748 & 119 \\
\hline $\mathrm{H}(21)$ & 1412 & 1783 & 6132 & 79 \\
\hline $\mathrm{H}(23)$ & -301 & 62 & 5785 & 41 \\
\hline $\mathrm{H}(24)$ & -262 & -1016 & 5765 & 55 \\
\hline $\mathrm{H}(25)$ & 1258 & -1342 & 7056 & 63 \\
\hline $\mathrm{H}(26)$ & 2128 & -474 & 7885 & 45 \\
\hline
\end{tabular}


Table S5f. Torsion angles $\left[^{\circ}\right]$ for $\mathbf{8 b}$.

\begin{tabular}{|c|c|}
\hline $\mathrm{O}(1)-\mathrm{Ni}(1)-\mathrm{P}(1)-\mathrm{C}(22)$ & $-167.8(3)$ \\
\hline $\mathrm{C}(1) \# 1-\mathrm{Ni}(1)-\mathrm{P}(1)-\mathrm{C}(22)$ & $-157.9(4)$ \\
\hline $\mathrm{O}(1) \# 1-\mathrm{Ni}(1)-\mathrm{P}(1)-\mathrm{C}(22)$ & $-157.9(4)$ \\
\hline $\mathrm{P}(1) \# 1-\mathrm{Ni}(1)-\mathrm{P}(1)-\mathrm{C}(22)$ & $15.7(2)$ \\
\hline $\mathrm{O}(1)-\mathrm{Ni}(1)-\mathrm{P}(1)-\mathrm{C}(10)$ & $70.9(3)$ \\
\hline $\mathrm{C}(1) \# 1-\mathrm{Ni}(1)-\mathrm{P}(1)-\mathrm{C}(10)$ & $80.8(4)$ \\
\hline $\mathrm{O}(1) \# 1-\mathrm{Ni}(1)-\mathrm{P}(1)-\mathrm{C}(10)$ & $80.8(4)$ \\
\hline $\mathrm{P}(1) \# 1-\mathrm{Ni}(1)-\mathrm{P}(1)-\mathrm{C}(10)$ & $-105.6(2)$ \\
\hline $\mathrm{O}(1)-\mathrm{Ni}(1)-\mathrm{P}(1)-\mathrm{C}(16)$ & $-48.2(3)$ \\
\hline $\mathrm{C}(1) \# 1-\mathrm{Ni}(1)-\mathrm{P}(1)-\mathrm{C}(16)$ & $-38.3(4)$ \\
\hline $\mathrm{O}(1) \# 1-\mathrm{Ni}(1)-\mathrm{P}(1)-\mathrm{C}(16)$ & $-38.3(4)$ \\
\hline $\mathrm{P}(1) \# 1-\mathrm{Ni}(1)-\mathrm{P}(1)-\mathrm{C}(16)$ & $135.4(2)$ \\
\hline $\mathrm{C}(1) \# 1-\mathrm{Ni}(1)-\mathrm{O}(1)-\mathrm{C}(2)$ & $106(4)$ \\
\hline $\mathrm{O}(1) \# 1-\mathrm{Ni}(1)-\mathrm{O}(1)-\mathrm{C}(2)$ & $106(4)$ \\
\hline $\mathrm{P}(1)-\mathrm{Ni}(1)-\mathrm{O}(1)-\mathrm{C}(2)$ & $-82(4)$ \\
\hline $\mathrm{P}(1) \# 1-\mathrm{Ni}(1)-\mathrm{O}(1)-\mathrm{C}(2)$ & $92(4)$ \\
\hline $\mathrm{O}(1) \# 1-\mathrm{Ni}(1)-\mathrm{O}(1)-\mathrm{C}(1) \# 1$ & 0.0 \\
\hline $\mathrm{P}(1)-\mathrm{Ni}(1)-\mathrm{O}(1)-\mathrm{C}(1) \# 1$ & $172.3(4)$ \\
\hline $\mathrm{P}(1) \# 1-\mathrm{Ni}(1)-\mathrm{O}(1)-\mathrm{C}(1) \# 1$ & $-14.0(7)$ \\
\hline $\mathrm{C}(1) \# 1-\mathrm{Ni}(1)-\mathrm{O}(1)-\mathrm{O}(1) \# 1$ & 0.0 \\
\hline $\mathrm{P}(1)-\mathrm{Ni}(1)-\mathrm{O}(1)-\mathrm{O}(1) \# 1$ & $172.3(4)$ \\
\hline $\mathrm{P}(1) \# 1-\mathrm{Ni}(1)-\mathrm{O}(1)-\mathrm{O}(1) \# 1$ & $-14.0(7)$ \\
\hline $\mathrm{C}(1) \# 1-\mathrm{O}(1)-\mathrm{C}(2)-\mathrm{C}(3)$ & $168.7(8)$ \\
\hline $\mathrm{O}(1) \# 1-\mathrm{O}(1)-\mathrm{C}(2)-\mathrm{C}(3)$ & $168.7(8)$ \\
\hline $\mathrm{Ni}(1)-\mathrm{O}(1)-\mathrm{C}(2)-\mathrm{C}(3)$ & $67(4)$ \\
\hline $\mathrm{O}(1)-\mathrm{C}(2)-\mathrm{C}(3)-\mathrm{C}(4)$ & $-107.4(10)$ \\
\hline$C(2)-C(3)-C(4)-C(5)$ & $178.3(11)$ \\
\hline$C(3)-C(4)-C(5)-C(6)$ & $179.8(11)$ \\
\hline $\mathrm{C}(7) \# 1-\mathrm{C}(7)-\mathrm{C}(8)-\mathrm{C}(9)$ & $-0.2(12)$ \\
\hline $\mathrm{C}(7)-\mathrm{C}(8)-\mathrm{C}(9)-\mathrm{C}(9) \# 1$ & $-1.0(17)$ \\
\hline$C(22)-P(1)-C(10)-C(11)$ & $75.5(6)$ \\
\hline$C(16)-P(1)-C(10)-C(11)$ & $-28.7(7)$ \\
\hline $\mathrm{Ni}(1)-\mathrm{P}(1)-\mathrm{C}(10)-\mathrm{C}(11)$ & $-154.4(5)$ \\
\hline $\mathrm{C}(22)-\mathrm{P}(1)-\mathrm{C}(10)-\mathrm{C}(15)$ & $-107.4(5)$ \\
\hline
\end{tabular}




\begin{tabular}{|c|c|}
\hline$C(16)-P(1)-C(10)-C(15)$ & $148.4(5)$ \\
\hline Ni(1)-P(1)-C(10)-C(15) & $22.6(6)$ \\
\hline$C(15)-C(10)-C(11)-C(12)$ & $-0.2(11)$ \\
\hline$P(1)-C(10)-C(11)-C(12)$ & $176.8(6)$ \\
\hline$C(10)-C(11)-C(12)-C(13)$ & $0.8(12)$ \\
\hline$C(11)-C(12)-C(13)-C(14)$ & $0.1(13)$ \\
\hline$C(12)-C(13)-C(14)-C(15)$ & $-1.6(12)$ \\
\hline$C(13)-C(14)-C(15)-C(10)$ & $2.2(11)$ \\
\hline$C(11)-C(10)-C(15)-C(14)$ & $-1.3(10)$ \\
\hline$P(1)-C(10)-C(15)-C(14)$ & $-178.5(5)$ \\
\hline$C(22)-P(1)-C(16)-C(21)$ & $150.6(6)$ \\
\hline $\mathrm{C}(10)-\mathrm{P}(1)-\mathrm{C}(16)-\mathrm{C}(21)$ & $-102.3(6)$ \\
\hline Ni(1)-P(1)-C(16)-C(21) & $20.7(6)$ \\
\hline $\mathrm{C}(22)-\mathrm{P}(1)-\mathrm{C}(16)-\mathrm{C}(17)$ & $-27.8(5)$ \\
\hline $\mathrm{C}(10)-\mathrm{P}(1)-\mathrm{C}(16)-\mathrm{C}(17)$ & $79.3(5)$ \\
\hline Ni(1)-P(1)-C(16)-C(17) & $-157.7(4)$ \\
\hline$C(21)-C(16)-C(17)-C(18)$ & $3.6(9)$ \\
\hline $\mathrm{P}(1)-\mathrm{C}(16)-\mathrm{C}(17)-\mathrm{C}(18)$ & $-178.0(5)$ \\
\hline $\mathrm{C}(16)-\mathrm{C}(17)-\mathrm{C}(18)-\mathrm{C}(19)$ & $-2.2(10)$ \\
\hline $\mathrm{C}(17)-\mathrm{C}(18)-\mathrm{C}(19)-\mathrm{C}(20)$ & $-3.2(13)$ \\
\hline$C(18)-C(19)-C(20)-C(21)$ & $7.1(16)$ \\
\hline $\mathrm{C}(17)-\mathrm{C}(16)-\mathrm{C}(21)-\mathrm{C}(20)$ & $0.4(13)$ \\
\hline $\mathrm{P}(1)-\mathrm{C}(16)-\mathrm{C}(21)-\mathrm{C}(20)$ & $-178.0(8)$ \\
\hline$C(19)-C(20)-C(21)-C(16)$ & $-5.7(16)$ \\
\hline $\mathrm{C}(10)-\mathrm{P}(1)-\mathrm{C}(22)-\mathrm{C}(26)$ & $-0.9(6)$ \\
\hline $\mathrm{C}(16)-\mathrm{P}(1)-\mathrm{C}(22)-\mathrm{C}(26)$ & $106.5(5)$ \\
\hline Ni(1)-P(1)-C(22)-C(26) & $-126.0(5)$ \\
\hline $\mathrm{C}(10)-\mathrm{P}(1)-\mathrm{C}(22)-\mathrm{C}(23)$ & $178.1(4)$ \\
\hline $\mathrm{C}(16)-\mathrm{P}(1)-\mathrm{C}(22)-\mathrm{C}(23)$ & $-74.5(5)$ \\
\hline Ni(1)-P(1)-C(22)-C(23) & $53.0(5)$ \\
\hline $\mathrm{C}(10)-\mathrm{P}(1)-\mathrm{C}(22)-\mathrm{Fe}(1)$ & $91.3(4)$ \\
\hline $\mathrm{C}(16)-\mathrm{P}(1)-\mathrm{C}(22)-\mathrm{Fe}(1)$ & $-161.2(3)$ \\
\hline $\mathrm{Ni}(1)-\mathrm{P}(1)-\mathrm{C}(22)-\mathrm{Fe}(1)$ & $-33.8(4)$ \\
\hline $\mathrm{C}(22) \# 1-\mathrm{Fe}(1)-\mathrm{C}(22)-\mathrm{C}(26)$ & $142.8(4)$ \\
\hline $\mathrm{C}(23) \# 1-\mathrm{Fe}(1)-\mathrm{C}(22)-\mathrm{C}(26)$ & $98.4(4)$ \\
\hline $\mathrm{C}(23)-\mathrm{Fe}(1)-\mathrm{C}(22)-\mathrm{C}(26)$ & $-118.9(5)$ \\
\hline
\end{tabular}




\begin{tabular}{|c|c|}
\hline $\mathrm{C}(26) \# 1-\mathrm{Fe}(1)-\mathrm{C}(22)-\mathrm{C}(26)$ & $179.8(3)$ \\
\hline $\mathrm{C}(24)-\mathrm{Fe}(1)-\mathrm{C}(22)-\mathrm{C}(26)$ & $-81.3(4)$ \\
\hline $\mathrm{C}(24) \# 1-\mathrm{Fe}(1)-\mathrm{C}(22)-\mathrm{C}(26)$ & $62.2(5)$ \\
\hline $\mathrm{C}(25)-\mathrm{Fe}(1)-\mathrm{C}(22)-\mathrm{C}(26)$ & $-37.2(4)$ \\
\hline $\mathrm{C}(25) \# 1-\mathrm{Fe}(1)-\mathrm{C}(22)-\mathrm{C}(26)$ & $140(9)$ \\
\hline $\mathrm{C}(22) \# 1-\mathrm{Fe}(1)-\mathrm{C}(22)-\mathrm{C}(23)$ & $-98.3(3)$ \\
\hline $\mathrm{C}(23) \# 1-\mathrm{Fe}(1)-\mathrm{C}(22)-\mathrm{C}(23)$ & $-142.8(4)$ \\
\hline $\mathrm{C}(26) \# 1-\mathrm{Fe}(1)-\mathrm{C}(22)-\mathrm{C}(23)$ & $-61.3(5)$ \\
\hline $\mathrm{C}(26)-\mathrm{Fe}(1)-\mathrm{C}(22)-\mathrm{C}(23)$ & $118.9(5)$ \\
\hline $\mathrm{C}(24)-\mathrm{Fe}(1)-\mathrm{C}(22)-\mathrm{C}(23)$ & $37.5(4)$ \\
\hline $\mathrm{C}(24) \# 1-\mathrm{Fe}(1)-\mathrm{C}(22)-\mathrm{C}(23)$ & $-178.9(4)$ \\
\hline $\mathrm{C}(25)-\mathrm{Fe}(1)-\mathrm{C}(22)-\mathrm{C}(23)$ & $81.7(4)$ \\
\hline $\mathrm{C}(25) \# 1-\mathrm{Fe}(1)-\mathrm{C}(22)-\mathrm{C}(23)$ & $-101(10)$ \\
\hline $\mathrm{C}(22) \# 1-\mathrm{Fe}(1)-\mathrm{C}(22)-\mathrm{P}(1)$ & $17.9(2)$ \\
\hline $\mathrm{C}(23) \# 1-\mathrm{Fe}(1)-\mathrm{C}(22)-\mathrm{P}(1)$ & $-26.6(4)$ \\
\hline $\mathrm{C}(23)-\mathrm{Fe}(1)-\mathrm{C}(22)-\mathrm{P}(1)$ & $116.2(5)$ \\
\hline $\mathrm{C}(26) \# 1-\mathrm{Fe}(1)-\mathrm{C}(22)-\mathrm{P}(1)$ & $54.8(5)$ \\
\hline $\mathrm{C}(26)-\mathrm{Fe}(1)-\mathrm{C}(22)-\mathrm{P}(1)$ & $-124.9(5)$ \\
\hline $\mathrm{C}(24)-\mathrm{Fe}(1)-\mathrm{C}(22)-\mathrm{P}(1)$ & $153.7(4)$ \\
\hline $\mathrm{C}(24) \# 1-\mathrm{Fe}(1)-\mathrm{C}(22)-\mathrm{P}(1)$ & $-62.7(5)$ \\
\hline $\mathrm{C}(25)-\mathrm{Fe}(1)-\mathrm{C}(22)-\mathrm{P}(1)$ & $-162.2(4)$ \\
\hline $\mathrm{C}(25) \# 1-\mathrm{Fe}(1)-\mathrm{C}(22)-\mathrm{P}(1)$ & $15(10)$ \\
\hline $\mathrm{C}(26)-\mathrm{C}(22)-\mathrm{C}(23)-\mathrm{C}(24)$ & $-0.4(6)$ \\
\hline $\mathrm{P}(1)-\mathrm{C}(22)-\mathrm{C}(23)-\mathrm{C}(24)$ & $-179.5(4)$ \\
\hline $\mathrm{Fe}(1)-\mathrm{C}(22)-\mathrm{C}(23)-\mathrm{C}(24)$ & $-60.0(4)$ \\
\hline $\mathrm{C}(26)-\mathrm{C}(22)-\mathrm{C}(23)-\mathrm{Fe}(1)$ & $59.6(4)$ \\
\hline $\mathrm{P}(1)-\mathrm{C}(22)-\mathrm{C}(23)-\mathrm{Fe}(1)$ & $-119.6(4)$ \\
\hline $\mathrm{C}(22)-\mathrm{Fe}(1)-\mathrm{C}(23)-\mathrm{C}(24)$ & 119.1(5) \\
\hline $\mathrm{C}(22) \# 1-\mathrm{Fe}(1)-\mathrm{C}(23)-\mathrm{C}(24)$ & $-143.3(4)$ \\
\hline $\mathrm{C}(23) \# 1-\mathrm{Fe}(1)-\mathrm{C}(23)-\mathrm{C}(24)$ & $178.9(4)$ \\
\hline $\mathrm{C}(26) \# 1-\mathrm{Fe}(1)-\mathrm{C}(23)-\mathrm{C}(24)$ & $-99.3(4)$ \\
\hline $\mathrm{C}(26)-\mathrm{Fe}(1)-\mathrm{C}(23)-\mathrm{C}(24)$ & $81.1(4)$ \\
\hline $\mathrm{C}(24) \# 1-\mathrm{Fe}(1)-\mathrm{C}(23)-\mathrm{C}(24)$ & $-122(20)$ \\
\hline $\mathrm{C}(25)-\mathrm{Fe}(1)-\mathrm{C}(23)-\mathrm{C}(24)$ & $37.8(4)$ \\
\hline $\mathrm{C}(25) \# 1-\mathrm{Fe}(1)-\mathrm{C}(23)-\mathrm{C}(24)$ & $-63.2(6)$ \\
\hline $\mathrm{C}(22) \# 1-\mathrm{Fe}(1)-\mathrm{C}(23)-\mathrm{C}(22)$ & $97.7(4)$ \\
\hline
\end{tabular}




\begin{tabular}{|c|c|}
\hline $\mathrm{C}(23) \# 1-\mathrm{Fe}(1)-\mathrm{C}(23)-\mathrm{C}(22)$ & $59.9(3)$ \\
\hline $\mathrm{C}(26) \# 1-\mathrm{Fe}(1)-\mathrm{C}(23)-\mathrm{C}(22)$ & $141.7(3)$ \\
\hline $\mathrm{C}(26)-\mathrm{Fe}(1)-\mathrm{C}(23)-\mathrm{C}(22)$ & $-38.0(3)$ \\
\hline $\mathrm{C}(24)-\mathrm{Fe}(1)-\mathrm{C}(23)-\mathrm{C}(22)$ & $-119.1(5)$ \\
\hline $\mathrm{C}(24) \# 1-\mathrm{Fe}(1)-\mathrm{C}(23)-\mathrm{C}(22)$ & $119(20)$ \\
\hline $\mathrm{C}(25)-\mathrm{Fe}(1)-\mathrm{C}(23)-\mathrm{C}(22)$ & $-81.2(4)$ \\
\hline $\mathrm{C}(25) \# 1-\mathrm{Fe}(1)-\mathrm{C}(23)-\mathrm{C}(22)$ & $177.7(4)$ \\
\hline$C(22)-C(23)-C(24)-C(25)$ & $-0.1(7)$ \\
\hline $\mathrm{Fe}(1)-\mathrm{C}(23)-\mathrm{C}(24)-\mathrm{C}(25)$ & $-59.1(5)$ \\
\hline $\mathrm{C}(22)-\mathrm{C}(23)-\mathrm{C}(24)-\mathrm{Fe}(1)$ & $58.9(4)$ \\
\hline $\mathrm{C}(22)-\mathrm{Fe}(1)-\mathrm{C}(24)-\mathrm{C}(23)$ & $-38.5(3)$ \\
\hline $\mathrm{C}(22) \# 1-\mathrm{Fe}(1)-\mathrm{C}(24)-\mathrm{C}(23)$ & $58.3(5)$ \\
\hline $\mathrm{C}(23) \# 1-\mathrm{Fe}(1)-\mathrm{C}(24)-\mathrm{C}(23)$ & $-60(20)$ \\
\hline $\mathrm{C}(26) \# 1-\mathrm{Fe}(1)-\mathrm{C}(24)-\mathrm{C}(23)$ & $96.9(4)$ \\
\hline $\mathrm{C}(26)-\mathrm{Fe}(1)-\mathrm{C}(24)-\mathrm{C}(23)$ & $-82.7(4)$ \\
\hline $\mathrm{C}(24) \# 1-\mathrm{Fe}(1)-\mathrm{C}(24)-\mathrm{C}(23)$ & $178.9(4)$ \\
\hline $\mathrm{C}(25)-\mathrm{Fe}(1)-\mathrm{C}(24)-\mathrm{C}(23)$ & $-119.5(6)$ \\
\hline $\mathrm{C}(25) \# 1-\mathrm{Fe}(1)-\mathrm{C}(24)-\mathrm{C}(23)$ & $140.4(4)$ \\
\hline $\mathrm{C}(22)-\mathrm{Fe}(1)-\mathrm{C}(24)-\mathrm{C}(25)$ & $81.0(4)$ \\
\hline $\mathrm{C}(22) \# 1-\mathrm{Fe}(1)-\mathrm{C}(24)-\mathrm{C}(25)$ & $177.7(4)$ \\
\hline $\mathrm{C}(23) \# 1-\mathrm{Fe}(1)-\mathrm{C}(24)-\mathrm{C}(25)$ & $60(20)$ \\
\hline $\mathrm{C}(23)-\mathrm{Fe}(1)-\mathrm{C}(24)-\mathrm{C}(25)$ & $119.5(6)$ \\
\hline $\mathrm{C}(26) \# 1-\mathrm{Fe}(1)-\mathrm{C}(24)-\mathrm{C}(25)$ & $-143.6(4)$ \\
\hline $\mathrm{C}(26)-\mathrm{Fe}(1)-\mathrm{C}(24)-\mathrm{C}(25)$ & $36.7(4)$ \\
\hline $\mathrm{C}(24) \# 1-\mathrm{Fe}(1)-\mathrm{C}(24)-\mathrm{C}(25)$ & $-61.6(4)$ \\
\hline $\mathrm{C}(25) \# 1-\mathrm{Fe}(1)-\mathrm{C}(24)-\mathrm{C}(25)$ & $-100.1(6)$ \\
\hline $\mathrm{C}(23)-\mathrm{C}(24)-\mathrm{C}(25)-\mathrm{C}(26)$ & $0.6(8)$ \\
\hline $\mathrm{Fe}(1)-\mathrm{C}(24)-\mathrm{C}(25)-\mathrm{C}(26)$ & $-58.0(5)$ \\
\hline $\mathrm{C}(23)-\mathrm{C}(24)-\mathrm{C}(25)-\mathrm{Fe}(1)$ & $58.5(4)$ \\
\hline $\mathrm{C}(22)-\mathrm{Fe}(1)-\mathrm{C}(25)-\mathrm{C}(26)$ & $38.0(4)$ \\
\hline $\mathrm{C}(22) \# 1-\mathrm{Fe}(1)-\mathrm{C}(25)-\mathrm{C}(26)$ & $41(10)$ \\
\hline $\mathrm{C}(23) \# 1-\mathrm{Fe}(1)-\mathrm{C}(25)-\mathrm{C}(26)$ & $-58.5(6)$ \\
\hline $\mathrm{C}(23)-\mathrm{Fe}(1)-\mathrm{C}(25)-\mathrm{C}(26)$ & $82.9(4)$ \\
\hline $\mathrm{C}(26) \# 1-\mathrm{Fe}(1)-\mathrm{C}(25)-\mathrm{C}(26)$ & $179.8(3)$ \\
\hline $\mathrm{C}(24)-\mathrm{Fe}(1)-\mathrm{C}(25)-\mathrm{C}(26)$ & $120.4(6)$ \\
\hline $\mathrm{C}(24) \# 1-\mathrm{Fe}(1)-\mathrm{C}(25)-\mathrm{C}(26)$ & $-97.4(4)$ \\
\hline
\end{tabular}




\begin{tabular}{|c|c|}
\hline $\mathrm{C}(25) \# 1-\mathrm{Fe}(1)-\mathrm{C}(25)-\mathrm{C}(26)$ & $-141.9(5)$ \\
\hline $\mathrm{C}(22)-\mathrm{Fe}(1)-\mathrm{C}(25)-\mathrm{C}(24)$ & $-82.4(4)$ \\
\hline $\mathrm{C}(22) \# 1-\mathrm{Fe}(1)-\mathrm{C}(25)-\mathrm{C}(24)$ & $-80(10)$ \\
\hline $\mathrm{C}(23) \# 1-\mathrm{Fe}(1)-\mathrm{C}(25)-\mathrm{C}(24)$ & $-178.9(4)$ \\
\hline $\mathrm{C}(23)-\mathrm{Fe}(1)-\mathrm{C}(25)-\mathrm{C}(24)$ & $-37.5(4)$ \\
\hline $\mathrm{C}(26) \# 1-\mathrm{Fe}(1)-\mathrm{C}(25)-\mathrm{C}(24)$ & $59.3(6)$ \\
\hline $\mathrm{C}(26)-\mathrm{Fe}(1)-\mathrm{C}(25)-\mathrm{C}(24)$ & $-120.4(6)$ \\
\hline $\mathrm{C}(24) \# 1-\mathrm{Fe}(1)-\mathrm{C}(25)-\mathrm{C}(24)$ & $142.2(5)$ \\
\hline $\mathrm{C}(25) \# 1-\mathrm{Fe}(1)-\mathrm{C}(25)-\mathrm{C}(24)$ & $97.7(4)$ \\
\hline $\mathrm{C}(24)-\mathrm{C}(25)-\mathrm{C}(26)-\mathrm{C}(22)$ & $-0.8(8)$ \\
\hline $\mathrm{Fe}(1)-\mathrm{C}(25)-\mathrm{C}(26)-\mathrm{C}(22)$ & $-59.0(4)$ \\
\hline $\mathrm{C}(24)-\mathrm{C}(25)-\mathrm{C}(26)-\mathrm{Fe}(1)$ & $58.2(5)$ \\
\hline $\mathrm{C}(23)-\mathrm{C}(22)-\mathrm{C}(26)-\mathrm{C}(25)$ & $0.7(7)$ \\
\hline $\mathrm{P}(1)-\mathrm{C}(22)-\mathrm{C}(26)-\mathrm{C}(25)$ & $179.8(5)$ \\
\hline $\mathrm{Fe}(1)-\mathrm{C}(22)-\mathrm{C}(26)-\mathrm{C}(25)$ & $60.1(5)$ \\
\hline $\mathrm{C}(23)-\mathrm{C}(22)-\mathrm{C}(26)-\mathrm{Fe}(1)$ & $-59.4(4)$ \\
\hline $\mathrm{P}(1)-\mathrm{C}(22)-\mathrm{C}(26)-\mathrm{Fe}(1)$ & $119.8(5)$ \\
\hline $\mathrm{C}(22)-\mathrm{Fe}(1)-\mathrm{C}(26)-\mathrm{C}(25)$ & $-119.4(5)$ \\
\hline $\mathrm{C}(22) \# 1-\mathrm{Fe}(1)-\mathrm{C}(26)-\mathrm{C}(25)$ & $-178.5(4)$ \\
\hline $\mathrm{C}(23) \# 1-\mathrm{Fe}(1)-\mathrm{C}(26)-\mathrm{C}(25)$ & $143.0(4)$ \\
\hline $\mathrm{C}(23)-\mathrm{Fe}(1)-\mathrm{C}(26)-\mathrm{C}(25)$ & $-81.0(4)$ \\
\hline $\mathrm{C}(26) \# 1-\mathrm{Fe}(1)-\mathrm{C}(26)-\mathrm{C}(25)$ & $-149(35)$ \\
\hline $\mathrm{C}(24)-\mathrm{Fe}(1)-\mathrm{C}(26)-\mathrm{C}(25)$ & $-37.3(4)$ \\
\hline $\mathrm{C}(24) \# 1-\mathrm{Fe}(1)-\mathrm{C}(26)-\mathrm{C}(25)$ & $99.4(5)$ \\
\hline $\mathrm{C}(25) \# 1-\mathrm{Fe}(1)-\mathrm{C}(26)-\mathrm{C}(25)$ & $62.1(8)$ \\
\hline $\mathrm{C}(22) \# 1-\mathrm{Fe}(1)-\mathrm{C}(26)-\mathrm{C}(22)$ & $-59.1(6)$ \\
\hline $\mathrm{C}(23) \# 1-\mathrm{Fe}(1)-\mathrm{C}(26)-\mathrm{C}(22)$ & $-97.6(4)$ \\
\hline $\mathrm{C}(23)-\mathrm{Fe}(1)-\mathrm{C}(26)-\mathrm{C}(22)$ & $38.4(3)$ \\
\hline $\mathrm{C}(26) \# 1-\mathrm{Fe}(1)-\mathrm{C}(26)-\mathrm{C}(22)$ & $-30(35)$ \\
\hline $\mathrm{C}(24)-\mathrm{Fe}(1)-\mathrm{C}(26)-\mathrm{C}(22)$ & $82.1(4)$ \\
\hline $\mathrm{C}(24) \# 1-\mathrm{Fe}(1)-\mathrm{C}(26)-\mathrm{C}(22)$ & $-141.2(4)$ \\
\hline $\mathrm{C}(25)-\mathrm{Fe}(1)-\mathrm{C}(26)-\mathrm{C}(22)$ & $119.4(5)$ \\
\hline $\mathrm{C}(25) \# 1-\mathrm{Fe}(1)-\mathrm{C}(26)-\mathrm{C}(22)$ & $-178.5(4)$ \\
\hline
\end{tabular}

Symmetry transformations used to generate equivalent atoms:

$\# 1-\mathrm{x}, \mathrm{y},-\mathrm{z}+3 / 2$ 
Table S6a. Sample and crystal data for $8 \mathrm{e}$.

\begin{tabular}{lll} 
Identification code & $\mathbf{8 e}$ \\
Chemical formula & $\mathrm{C}_{46} \mathrm{H}_{42} \mathrm{FeNiOP}_{2}$ & $787.29 \mathrm{~g} / \mathrm{mol}$ \\
Formula weight & $150(1) \mathrm{K}$ \\
Temperature & $0.71073 \AA$ & \\
Wavelength & $0.112 \times 0.238 \times 0.238 \mathrm{~mm}$ \\
Crystal size & clear intense orange prism \\
Crystal habit & monoclinic & \\
Crystal system & $\mathrm{P} 121 / \mathrm{c} 1$ & $\alpha=90^{\circ}$ \\
Space group & $\mathrm{a}=10.8641(4) \AA$ & $\beta=106.661(2)^{\circ}$ \\
Unit cell dimensions & $\mathrm{b}=19.3805(6) \AA$ & $\gamma=90^{\circ}$ \\
& $\mathrm{c}=18.5952(6) \AA$ & \\
\hline Volume & $3750.9(2) \AA^{3}$ & \\
Z & 4 & \\
Density (calculated) & $1.394 \mathrm{~g} / \mathrm{cm}^{3}$ & \\
Absorption coefficient & $1.010 \mathrm{~mm}^{-1}$ & \\
F(000) & 1640 &
\end{tabular}

Table S6b. Data collection and structure refinement for $8 \mathrm{e}$.

Theta range for data collection $\quad 2.10$ to $27.88^{\circ}$

Index ranges $\quad-13<=\mathrm{h}<=14,-25<=\mathrm{k}<=25,-24<=1<=22$

Reflections collected 93394

Independent reflections $\quad 8957[\mathrm{R}($ int $)=0.0441]$

Coverage of independent reflections $100.0 \%$

Absorption correction multi-scan

Max. and min. transmission $\quad 0.8950$ and 0.7950

Structure solution technique direct methods

Structure solution program $\quad$ SHELXT-2014/9 (Sheldrick, 2014) 


\begin{tabular}{|c|c|}
\hline Refinement method & Full-matrix least-squares on $\mathrm{F}^{2}$ \\
\hline Refinement program & SHELXL-2014/6 (Sheldrick, 2014) \\
\hline Function minimized & $\Sigma \mathrm{w}\left(\mathrm{F}_{\mathrm{o}}^{2}-\mathrm{F}_{\mathrm{c}}^{2}\right)^{2}$ \\
\hline Data / restraints / parameters & 8957 / 12 / 469 \\
\hline Goodness-of-fit on $F^{2}$ & 1.031 \\
\hline$\Delta / \sigma_{\max }$ & 0.002 \\
\hline \multirow[t]{2}{*}{ Final $\mathbf{R}$ indices } & 7198 data; $\mathrm{I}>2 \sigma(\mathrm{I}) \mathrm{R} 1=0.0338, \mathrm{wR} 2=0.0810$ \\
\hline & $\mathrm{R} 1=0.0484, \mathrm{wR} 2=0.0871$ \\
\hline \multirow{2}{*}{ Weighting scheme } & $\mathrm{w}=1 /\left[\sigma^{2}\left(\mathrm{~F}_{\mathrm{o}}^{2}\right)+(0.0377 \mathrm{P})^{2}+2.7129 \mathrm{P}\right]$ \\
\hline & where $\mathrm{P}=\left(\mathrm{F}_{\mathrm{o}}^{2}+2 \mathrm{~F}_{\mathrm{c}}^{2}\right) / 3$ \\
\hline Largest diff. peak and hole & 0.608 and $-0.415 \mathrm{e}^{-3}$ \\
\hline R.M.S. deviation from mean & $0.061 \mathrm{e}^{-3}$ \\
\hline
\end{tabular}

Table S6c. Atomic coordinates and equivalent isotropic atomic displacement parameters $\left(\AA^{2}\right)$ for $8 \mathrm{e}$.

$\mathrm{U}(\mathrm{eq})$ is defined as one third of the trace of the orthogonalized $\mathrm{U}_{\mathrm{ij}}$ tensor.

$\begin{array}{lllll} & \mathbf{x} / \mathbf{a} & \mathbf{y} / \mathbf{b} & \mathbf{z} / \mathbf{c} & \mathbf{U}(\mathbf{e q}) \\ \mathrm{N} 11 & 0.60303(2) & 0.31642(2) & 0.57406(2) & 0.02042(7) \\ \mathrm{Fe} 1 & 0.64931(3) & 0.29205(2) & 0.36581(2) & 0.02462(7) \\ \mathrm{P} 1 & 0.77199(5) & 0.35107(3) & 0.54663(3) & 0.02227(11) \\ \mathrm{P} 2 & 0.52961(5) & 0.22868(3) & 0.49743(3) & 0.02251(11) \\ \mathrm{O} 1 & 0.47344(13) & 0.33009(7) & 0.61982(7) & 0.0248(3) \\ \mathrm{C} 1 & 0.56661(18) & 0.37250(10) & 0.64779(10) & 0.0238(4) \\ \mathrm{C} 2 & 0.5897(2) & 0.42438(10) & 0.69850(11) & 0.0290(4) \\ \mathrm{C} 3 & 0.7249(2) & 0.45422(12) & 0.72276(13) & 0.0405(5) \\ \mathrm{C} 4 & 0.8009(3) & 0.43325(17) & 0.80260(18) & 0.0686(9) \\ \mathrm{C} 5 & 0.9412(6) & 0.4561(4) & 0.8145(5) & 0.110(3) \\ \mathrm{C} 5 \mathrm{~A} & 0.8882(7) & 0.5552(4) & 0.7441(4) & 0.086(2) \\ \mathrm{C} 6 & 0.7357(3) & 0.53065(14) & 0.71027(17) & 0.0555(7)\end{array}$




\begin{tabular}{|c|c|c|c|c|}
\hline & $\mathbf{x} / \mathbf{a}$ & $\mathbf{y} / \mathbf{b}$ & $\mathbf{z} / \mathbf{c}$ & $\mathrm{U}(\mathbf{e q})$ \\
\hline $\mathrm{C} 7$ & $0.4864(2)$ & $0.44891(11)$ & $0.72991(11)$ & $0.0314(4)$ \\
\hline $\mathrm{C} 8$ & $0.3600(2)$ & $0.42796(14)$ & $0.70034(14)$ & $0.0454(6)$ \\
\hline $\mathrm{C} 9$ & $0.2617(3)$ & $0.45132(17)$ & $0.72813(15)$ & $0.0563(7)$ \\
\hline $\mathrm{C} 10$ & $0.2860(3)$ & $0.49696(14)$ & $0.78641(15)$ & $0.0499(6)$ \\
\hline $\mathrm{C} 11$ & $0.4089(3)$ & $0.51924(14)$ & $0.81660(15)$ & $0.0515(7)$ \\
\hline $\mathrm{C} 12$ & $0.5086(3)$ & $0.49577(13)$ & $0.78954(14)$ & $0.0437(6)$ \\
\hline $\mathrm{C} 13$ & $0.78587(19)$ & $0.44496(10)$ & $0.54310(11)$ & $0.0260(4)$ \\
\hline $\mathrm{C} 14$ & $0.6744(2)$ & $0.48155(11)$ & $0.51002(12)$ & $0.0326(5)$ \\
\hline $\mathrm{C} 15$ & $0.6778(2)$ & $0.55259(12)$ & $0.50171(14)$ & $0.0410(5)$ \\
\hline $\mathrm{C} 16$ & $0.7909(3)$ & $0.58762(13)$ & $0.52759(16)$ & $0.0477(6)$ \\
\hline $\mathrm{C} 17$ & $0.9021(3)$ & $0.55187(13)$ & $0.56131(17)$ & $0.0510(7)$ \\
\hline $\mathrm{C} 18$ & $0.9003(2)$ & $0.48057(12)$ & $0.56849(14)$ & $0.0385(5)$ \\
\hline C19 & $0.92337(18)$ & $0.32085(10)$ & $0.61034(11)$ & $0.0252(4)$ \\
\hline $\mathrm{C} 20$ & $0.03621(19)$ & $0.31683(11)$ & $0.58940(13)$ & $0.0317(4)$ \\
\hline $\mathrm{C} 21$ & $0.1478(2)$ & $0.29120(13)$ & $0.63894(14)$ & $0.0413(5)$ \\
\hline $\mathrm{C} 22$ & $0.1473(2)$ & $0.26858(15)$ & $0.70911(14)$ & $0.0479(6)$ \\
\hline $\mathrm{C} 23$ & $0.0356(2)$ & $0.27153(15)$ & $0.73054(13)$ & $0.0467(6)$ \\
\hline $\mathrm{C} 24$ & $0.9240(2)$ & $0.29796(12)$ & $0.68143(12)$ & $0.0336(5)$ \\
\hline $\mathrm{C} 25$ & $0.37410(19)$ & $0.19515(10)$ & $0.50116(12)$ & $0.0275(4)$ \\
\hline $\mathrm{C} 26$ & $0.3643(2)$ & $0.17028(12)$ & $0.56947(13)$ & $0.0376(5)$ \\
\hline $\mathrm{C} 27$ & $0.2488(2)$ & $0.14446(13)$ & $0.57534(16)$ & $0.0455(6)$ \\
\hline $\mathrm{C} 28$ & $0.1429(2)$ & $0.14440(14)$ & $0.51434(18)$ & $0.0528(7)$ \\
\hline $\mathrm{C} 29$ & $0.1512(3)$ & $0.16894(18)$ & $0.4470(2)$ & $0.0672(9)$ \\
\hline $\mathrm{C} 30$ & $0.2675(2)$ & $0.19385(15)$ & $0.43998(16)$ & $0.0520(7)$ \\
\hline C31 & $0.62841(19)$ & $0.15088(10)$ & $0.51338(11)$ & $0.0271(4)$ \\
\hline $\mathrm{C} 32$ & $0.7497(2)$ & $0.15401(12)$ & $0.56428(13)$ & $0.0351(5)$ \\
\hline $\mathrm{C} 33$ & $0.8300(2)$ & $0.09682(13)$ & $0.57614(15)$ & $0.0455(6)$ \\
\hline $\mathrm{C} 34$ & $0.7881(3)$ & $0.03626(13)$ & $0.53779(15)$ & $0.0480(6)$ \\
\hline $\mathrm{C} 35$ & $0.6667(3)$ & $0.03222(12)$ & $0.48898(14)$ & $0.0433(6)$ \\
\hline
\end{tabular}




$\begin{array}{lllll} & \mathbf{x} / \mathbf{a} & \mathbf{y} / \mathbf{b} & \mathbf{z} / \mathbf{c} & \mathbf{U}(\mathbf{e q}) \\ \text { C36 } & 0.5865(2) & 0.08898(11) & 0.47644(12) & 0.0338(5) \\ \text { C37 } & 0.79091(18) & 0.32969(10) & 0.45481(11) & 0.0261(4) \\ \text { C38 } & 0.7707(2) & 0.37508(11) & 0.39143(11) & 0.0311(4) \\ \text { C39 } & 0.7938(2) & 0.33680(13) & 0.33122(12) & 0.0370(5) \\ \text { C40 } & 0.8273(2) & 0.26901(13) & 0.35569(12) & 0.0365(5) \\ \text { C41 } & 0.82553(19) & 0.26366(11) & 0.43185(12) & 0.0307(4) \\ \text { C42 } & 0.50482(18) & 0.25000(10) & 0.40013(10) & 0.0234(4) \\ \text { C43 } & 0.47136(18) & 0.31794(10) & 0.37177(11) & 0.0265(4) \\ \text { C44 } & 0.4733(2) & 0.31892(12) & 0.29541(11) & 0.0321(5) \\ \text { C45 } & 0.5080(2) & 0.25234(12) & 0.27668(12) & 0.0340(5) \\ \text { C46 } & 0.5272(2) & 0.20940(11) & 0.34055(11) & 0.0295(4)\end{array}$

Table S6d. Bond lengths ( $\AA$ ) for 8e.

$\begin{array}{llll}\text { Ni1-O1 } & 1.8611(12) & \text { Ni1-C1 } & 1.8785(18) \\ \text { Ni1-P1 } & 2.1488(5) & \text { Ni1-P2 } & 2.2150(5) \\ \text { Fe1-C42 } & 2.0273(18) & \text { Fe1-C43 } & 2.0308(19) \\ \text { Fe1-C41 } & 2.032(2) & \text { Fe1-C37 } & 2.044(2) \\ \text { Fe1-C40 } & 2.045(2) & \text { Fe1-C46 } & 2.047(2) \\ \text { Fe1-C38 } & 2.048(2) & \text { Fe1-C44 } & 2.051(2) \\ \text { Fe1-C39 } & 2.052(2) & \text { Fe1-C45 } & 2.058(2) \\ \text { P1-C37 } & 1.825(2) & \text { P1-C19 } & 1.826(2) \\ \text { P1-C13 } & 1.829(2) & \text { P2-C42 } & 1.7994(19) \\ \text { P2-C31 } & 1.825(2) & \text { P2-C25 } & 1.830(2) \\ \text { O1-C1 } & 1.292(2) & \text { C1-C2 } & 1.352(3) \\ \text { C2-C7 } & 1.485(3) & \text { C2-C3 } & 1.521(3) \\ \text { C3-C6 } & 1.509(3) & \text { C3-C4 } & 1.532(4) \\ \text { C3-H3 } & 1.0 & \text { C4-C5 } & 1.541(7) \\ \text { C4-H4A } & 0.99 & \text { C4-H4B } & 0.99\end{array}$




\begin{tabular}{|c|c|c|c|}
\hline $\mathrm{C} 4-\mathrm{H} 4 \mathrm{C}$ & 0.98 & C4-H4D & 0.98 \\
\hline C4-H4E & 0.98 & C5-H5A & 0.98 \\
\hline C5-H5B & 0.98 & C5-H5C & 0.98 \\
\hline C5A-C6 & $1.665(6)$ & C5A-H5A1 & 0.98 \\
\hline C5A-H5A2 & 0.98 & C5A-H5A3 & 0.98 \\
\hline C6-H6A & 0.98 & C6-H6B & 0.98 \\
\hline C6-H6C & 0.98 & C6-H6D & 0.99 \\
\hline C6-H6E & 0.99 & $\mathrm{C} 7-\mathrm{C} 8$ & $1.385(3)$ \\
\hline $\mathrm{C} 7-\mathrm{C} 12$ & $1.400(3)$ & C8-C9 & $1.388(3)$ \\
\hline C8-H8 & 0.95 & C9-C10 & $1.365(4)$ \\
\hline C9-H9 & 0.95 & C10-C11 & $1.362(4)$ \\
\hline C10-H10 & 0.95 & $\mathrm{C} 11-\mathrm{C} 12$ & $1.395(3)$ \\
\hline C11-H11 & 0.95 & C12-H12 & 0.95 \\
\hline C13-C18 & $1.381(3)$ & $\mathrm{C} 13-\mathrm{C} 14$ & $1.386(3)$ \\
\hline C14-C15 & $1.387(3)$ & C14-H14 & 0.95 \\
\hline $\mathrm{C} 15-\mathrm{C} 16$ & $1.365(4)$ & $\mathrm{C} 15-\mathrm{H} 15$ & 0.95 \\
\hline C16-C17 & $1.378(4)$ & C16-H16 & 0.95 \\
\hline C17-C18 & $1.389(3)$ & C17-H17 & 0.95 \\
\hline C18-H18 & 0.95 & C19-C20 & $1.391(3)$ \\
\hline C19-C24 & $1.393(3)$ & $\mathrm{C} 20-\mathrm{C} 21$ & $1.388(3)$ \\
\hline C20-H20 & 0.95 & $\mathrm{C} 21-\mathrm{C} 22$ & $1.378(4)$ \\
\hline $\mathrm{C} 21-\mathrm{H} 21$ & 0.95 & $\mathrm{C} 22-\mathrm{C} 23$ & $1.383(3)$ \\
\hline C22-H22 & 0.95 & C23-C24 & $1.390(3)$ \\
\hline C23-H23 & 0.95 & C24-H24 & 0.95 \\
\hline C25-C30 & $1.372(3)$ & $\mathrm{C} 25-\mathrm{C} 26$ & 1.391(3) \\
\hline C26-C27 & $1.384(3)$ & $\mathrm{C} 26-\mathrm{H} 26$ & 0.95 \\
\hline $\mathrm{C} 27-\mathrm{C} 28$ & $1.365(4)$ & $\mathrm{C} 27-\mathrm{H} 27$ & 0.95 \\
\hline C28-C29 & $1.367(4)$ & C28-H28 & 0.95 \\
\hline C29-C30 & $1.393(4)$ & C29-H29 & 0.95 \\
\hline C30-H30 & 0.95 & C31-C32 & $1.385(3)$ \\
\hline
\end{tabular}




$\begin{array}{llll}\text { C31-C36 } & 1.392(3) & \mathrm{C} 32-\mathrm{C} 33 & 1.389(3) \\ \mathrm{C} 32-\mathrm{H} 32 & 0.95 & \mathrm{C} 33-\mathrm{C} 34 & 1.381(4) \\ \mathrm{C} 33-\mathrm{H} 33 & 0.95 & \mathrm{C} 34-\mathrm{C} 35 & 1.373(4) \\ \mathrm{C} 34-\mathrm{H} 34 & 0.95 & \mathrm{C} 35-\mathrm{C} 36 & 1.381(3) \\ \mathrm{C} 35-\mathrm{H} 35 & 0.95 & \mathrm{C} 36-\mathrm{H} 36 & 0.95 \\ \mathrm{C} 37-\mathrm{C} 41 & 1.433(3) & \mathrm{C} 37-\mathrm{C} 38 & 1.436(3) \\ \mathrm{C} 38-\mathrm{C} 39 & 1.424(3) & \mathrm{C} 38-\mathrm{H} 38 & 1.0 \\ \mathrm{C} 39-\mathrm{C} 40 & 1.404(3) & \mathrm{C} 39-\mathrm{H} 39 & 1.0 \\ \mathrm{C} 40-\mathrm{C} 41 & 1.426(3) & \mathrm{C} 40-\mathrm{H} 40 & 1.0 \\ \mathrm{C} 41-\mathrm{H} 41 & 1.0 & \mathrm{C} 42-\mathrm{C} 43 & 1.426(3) \\ \mathrm{C} 42-\mathrm{C} 46 & 1.435(3) & \mathrm{C} 43-\mathrm{C} 44 & 1.426(3) \\ \mathrm{C} 43-\mathrm{H} 43 & 1.0 & \mathrm{C} 44-\mathrm{C} 45 & 1.415(3) \\ \mathrm{C} 44-\mathrm{H} 44 & 1.0 & \mathrm{C} 45-\mathrm{C} 46 & 1.416(3) \\ \mathrm{C} 45-\mathrm{H} 45 & 1.0 & \mathrm{C} 46-\mathrm{H} 46 & 1.0\end{array}$

Table S6e. Bond angles $\left(^{\circ}\right)$ for 8 e.

$\begin{array}{llll}\text { O1-Ni1-C1 } & 40.41(7) & \text { O1-Ni1-P1 } & 150.89(5) \\ \text { C1-Ni1-P1 } & 111.44(6) & \text { O1-Ni1-P2 } & 102.90(4) \\ \text { C1-Ni1-P2 } & 143.25(6) & \text { P1-Ni1-P2 } & 105.18(2) \\ \text { C42-Fe1-C43 } & 41.15(8) & \text { C42-Fe1-C41 } & 112.41(8) \\ \text { C43-Fe1-C41 } & 141.33(8) & \text { C42-Fe1-C37 } & 111.07(8) \\ \text { C43-Fe1-C37 } & 112.02(8) & \text { C41-Fe1-C37 } & 41.17(8) \\ \text { C42-Fe1-C40 } & 141.17(9) & \text { C43-Fe1-C40 } & 177.36(9) \\ \text { C41-Fe1-C40 } & 40.93(8) & \text { C37-Fe1-C40 } & 68.92(8) \\ \text { C42-Fe1-C46 } & 41.25(7) & \text { C43-Fe1-C46 } & 68.98(8) \\ \text { C41-Fe1-C46 } & 111.30(9) & \text { C37-Fe1-C46 } & 138.85(8) \\ \text { C40-Fe1-C46 } & 112.07(9) & \text { C42-Fe1-C38 } & 138.37(8) \\ \text { C43-Fe1-C38 } & 110.64(9) & \text { C41-Fe1-C38 } & 68.90(9) \\ \text { C37-Fe1-C38 } & 41.09(8) & \text { C40-Fe1-C38 } & 68.31(9) \\ \text { C46-Fe1-C38 } & 179.60(9) & \text { C42-Fe1-C44 } & 68.82(8) \\ & & & \text { S111 }\end{array}$




\begin{tabular}{|c|c|c|c|}
\hline $\mathrm{C} 43-\mathrm{Fe} 1-\mathrm{C} 44$ & $40.90(8)$ & C41-Fe1-C44 & $177.62(8)$ \\
\hline C37-Fe1-C44 & $140.70(8)$ & $\mathrm{C} 40-\mathrm{Fe} 1-\mathrm{C} 44$ & $136.87(9)$ \\
\hline C46-Fe1-C44 & $68.15(9)$ & $\mathrm{C} 38-\mathrm{Fe} 1-\mathrm{C} 44$ & $111.66(9)$ \\
\hline C42-Fe1-C39 & $178.69(9)$ & C43-Fe1-C39 & 137.62(9) \\
\hline C41-Fe1-C39 & 68.33(9) & C37-Fe1-C39 & $68.71(8)$ \\
\hline C40-Fe1-C39 & $40.07(10)$ & C46-Fe1-C39 & 139.71(9) \\
\hline C38-Fe1-C39 & $40.66(8)$ & C44-Fe1-C39 & $110.48(9)$ \\
\hline $\mathrm{C} 42-\mathrm{Fe} 1-\mathrm{C} 45$ & $68.72(8)$ & $\mathrm{C} 43-\mathrm{Fe} 1-\mathrm{C} 45$ & $68.55(8)$ \\
\hline C41-Fe1-C45 & $137.85(9)$ & C37-Fe1-C45 & $178.95(9)$ \\
\hline $\mathrm{C} 40-\mathrm{Fe} 1-\mathrm{C} 45$ & $110.54(9)$ & $\mathrm{C} 46-\mathrm{Fe} 1-\mathrm{C} 45$ & $40.35(9)$ \\
\hline C38-Fe1-C45 & 139.71(9) & C44-Fe1-C45 & $40.30(9)$ \\
\hline C39-Fe1-C45 & $111.52(9)$ & C37-P1-C19 & $102.24(9)$ \\
\hline C37-P1-C13 & $99.19(9)$ & C19-P1-C13 & 105.92(9) \\
\hline C37-P1-Ni1 & $118.96(7)$ & C19-P1-Ni1 & $114.68(7)$ \\
\hline C13-P1-Ni1 & $113.85(6)$ & C42-P2-C31 & $105.35(9)$ \\
\hline C42-P2-C25 & 104.24(9) & C31-P2-C25 & 101.94(9) \\
\hline C42-P2-Ni1 & $113.14(6)$ & C31-P2-Ni1 & $116.30(7)$ \\
\hline C25-P2-Ni1 & $114.48(7)$ & C1-O1-Ni1 & $70.52(10)$ \\
\hline $\mathrm{O} 1-\mathrm{C} 1-\mathrm{C} 2$ & $136.18(18)$ & O1-C1-Nil & $69.07(10)$ \\
\hline C2-C1-Ni1 & $154.63(16)$ & $\mathrm{C} 1-\mathrm{C} 2-\mathrm{C} 7$ & $120.16(19)$ \\
\hline $\mathrm{C} 1-\mathrm{C} 2-\mathrm{C} 3$ & $117.39(18)$ & $\mathrm{C} 7-\mathrm{C} 2-\mathrm{C} 3$ & $122.43(18)$ \\
\hline C6-C3-C2 & $116.0(2)$ & $\mathrm{C} 6-\mathrm{C} 3-\mathrm{C} 4$ & $111.4(2)$ \\
\hline $\mathrm{C} 2-\mathrm{C} 3-\mathrm{C} 4$ & $113.1(2)$ & $\mathrm{C} 6-\mathrm{C} 3-\mathrm{H} 3$ & 105.0 \\
\hline $\mathrm{C} 2-\mathrm{C} 3-\mathrm{H} 3$ & 105.0 & $\mathrm{C} 4-\mathrm{C} 3-\mathrm{H} 3$ & 105.0 \\
\hline $\mathrm{C} 3-\mathrm{C} 4-\mathrm{C} 5$ & $107.4(4)$ & C3-C4-H4A & 110.2 \\
\hline C5-C4-H4A & 110.2 & C3-C4-H4B & 110.2 \\
\hline C5-C4-H4B & 110.2 & H4A-C4-H4B & 108.5 \\
\hline $\mathrm{C} 3-\mathrm{C} 4-\mathrm{H} 4 \mathrm{C}$ & 109.5 & C3-C4-H4D & 109.5 \\
\hline H4C-C4-H4D & 109.5 & C3-C4-H4E & 109.5 \\
\hline H4C-C4-H4E & 109.5 & H4D-C4-H4E & 109.5 \\
\hline
\end{tabular}




\begin{tabular}{|c|c|c|c|}
\hline C4-C5-H5A & 109.5 & C4-C5-H5B & 109.5 \\
\hline H5A-C5-H5B & 109.5 & $\mathrm{C} 4-\mathrm{C} 5-\mathrm{H} 5 \mathrm{C}$ & 109.5 \\
\hline $\mathrm{H} 5 \mathrm{~A}-\mathrm{C} 5-\mathrm{H} 5 \mathrm{C}$ & 109.5 & H5B-C5-H5C & 109.5 \\
\hline C6-C5A-H5A1 & 109.5 & C6-C5A-H5A2 & 109.5 \\
\hline H5A1-C5A-H5A2 & 109.5 & C6-C5A-H5A3 & 109.5 \\
\hline H5A1-C5A-H5A3 & 109.5 & H5A2-C5A-H5A3 & 109.5 \\
\hline C3-C6-C5A & 109.6(4) & C3-C6-H6A & 109.5 \\
\hline C3-C6-H6B & 109.5 & H6A-C6-H6B & 109.5 \\
\hline C3-C6-H6C & 109.5 & H6A-C6-H6C & 109.5 \\
\hline H6B-C6-H6C & 109.5 & C3-C6-H6D & 109.8 \\
\hline C5A-C6-H6D & 109.8 & C3-C6-H6E & 109.8 \\
\hline C5A-C6-H6E & 109.8 & H6D-C6-H6E & 108.2 \\
\hline $\mathrm{C} 8-\mathrm{C} 7-\mathrm{C} 12$ & $115.5(2)$ & $\mathrm{C} 8-\mathrm{C} 7-\mathrm{C} 2$ & $121.68(19)$ \\
\hline $\mathrm{C} 12-\mathrm{C} 7-\mathrm{C} 2$ & $122.8(2)$ & $\mathrm{C} 7-\mathrm{C} 8-\mathrm{C} 9$ & $122.5(2)$ \\
\hline C7-C8-H8 & 118.8 & $\mathrm{C} 9-\mathrm{C} 8-\mathrm{H} 8$ & 118.8 \\
\hline $\mathrm{C} 10-\mathrm{C} 9-\mathrm{C} 8$ & $120.8(3)$ & C10-C9-H9 & 119.6 \\
\hline C8-C9-H9 & 119.6 & $\mathrm{C} 11-\mathrm{C} 10-\mathrm{C} 9$ & $118.4(2)$ \\
\hline C11-C10-H10 & 120.8 & C9-C10-H10 & 120.8 \\
\hline C10-C11-C12 & $121.2(2)$ & C10-C11-H11 & 119.4 \\
\hline C12-C11-H11 & 119.4 & $\mathrm{C} 11-\mathrm{C} 12-\mathrm{C} 7$ & $121.5(2)$ \\
\hline C11-C12-H12 & 119.3 & C7-C12-H12 & 119.3 \\
\hline C18-C13-C14 & $118.8(2)$ & C18-C13-P1 & $124.08(16)$ \\
\hline C14-C13-P1 & $117.04(15)$ & $\mathrm{C} 13-\mathrm{C} 14-\mathrm{C} 15$ & $120.6(2)$ \\
\hline C13-C14-H14 & 119.7 & C15-C14-H14 & 119.7 \\
\hline C16-C15-C14 & $120.3(2)$ & $\mathrm{C} 16-\mathrm{C} 15-\mathrm{H} 15$ & 119.8 \\
\hline C14-C15-H15 & 119.8 & C15-C16-C17 & $119.6(2)$ \\
\hline C15-C16-H16 & 120.2 & C17-C16-H16 & 120.2 \\
\hline C16-C17-C18 & $120.6(2)$ & C16-C17-H17 & 119.7 \\
\hline C18-C17-H17 & 119.7 & $\mathrm{C} 13-\mathrm{C} 18-\mathrm{C} 17$ & $120.0(2)$ \\
\hline C13-C18-H18 & 120.0 & C17-C18-H18 & 120.0 \\
\hline
\end{tabular}




$\begin{array}{llll}\text { C20-C19-C24 } & 118.91(19) & \text { C20-C19-P1 } & 122.58(16) \\ \text { C24-C19-P1 } & 118.44(15) & \text { C21-C20-C19 } & 120.5(2) \\ \text { C21-C20-H20 } & 119.8 & \text { C19-C20-H20 } & 119.8 \\ \text { C22-C21-C20 } & 120.2(2) & \text { C22-C21-H21 } & 119.9 \\ \text { C20-C21-H21 } & 119.9 & \text { C21-C22-C23 } & 120.1(2) \\ \text { C21-C22-H22 } & 119.9 & \text { C23-C22-H22 } & 119.9 \\ \text { C22-C23-C24 } & 119.9(2) & \text { C22-C23-H23 } & 120.0 \\ \text { C24-C23-H23 } & 120.0 & \text { C23-C24-C19 } & 120.4(2) \\ \text { C23-C24-H24 } & 119.8 & \text { C19-C24-H24 } & 119.8 \\ \text { C30-C25-C26 } & 118.9(2) & \text { C30-C25-P2 } & 122.99(17) \\ \text { C26-C25-P2 } & 118.08(17) & \text { C27-C26-C25 } & 120.4(2) \\ \text { C27-C26-H26 } & 119.8 & \text { C25-C26-H26 } & 119.8 \\ \text { C28-C27-C26 } & 120.2(2) & \text { C28-C27-H27 } & 119.9 \\ \text { C26-C27-H27 } & 119.9 & \text { C27-C28-C29 } & 120.0(2) \\ \text { C38-C37-P1 } & 126.65(16) & \text { C41-C37-Fe1 } & 68.96(11) \\ \text { C27-C28-H28 } & 120.0 & \text { C29-C28-H28 } & 120.0 \\ \text { C28-C29-C30 } & 120.4(3) & \text { C28-C29-H29 } & 119.8 \\ \text { C30-C29-H29 } & 119.8 & \text { C25-C30-C29 } & 120.1(3) \\ \text { C25-C30-H30 } & 119.9 & \text { C29-C30-H30 } & 119.9 \\ \text { C32-C31-C36 } & 119.2(2) & \text { C32-C31-P2 } & 118.33(16) \\ \text { C36-C31-P2 } & 122.52(17) & \text { C31-C32-C33 } & 120.3(2) \\ \text { C31-C32-H32 } & 119.8 & \text { C33-C32-H32 } & 119.8 \\ \text { C34-C33-C32 } & 119.8(2) & \text { C34-C33-H33 } & 120.1 \\ \text { C32-C33-H33 } & 120.1 & \text { C35-C34-C33 } & 120.1(2) \\ \text { C35-H34 } & 119.9 & \text { C33-C34-H34 } & 119.9 \\ \text { C35-C36 } & 120.4(2) & \text { C34-C35-H35 } & 119.8 \\ \text { C36 } 350.31(10)\end{array}$




\begin{tabular}{|c|c|c|c|}
\hline C39-C38-C37 & 107.82(19) & C39-C38-Fe1 & $69.82(12)$ \\
\hline C37-C38-Fe1 & $69.31(11)$ & C39-C38-H38 & 126.1 \\
\hline C37-C38-H38 & 126.1 & Fe1-C38-H38 & 126.1 \\
\hline C40-C39-C38 & $108.70(19)$ & C40-C39-Fe1 & $69.71(12)$ \\
\hline C38-C39-Fe1 & $69.52(11)$ & C40-C39-H39 & 125.6 \\
\hline C38-C39-H39 & 125.6 & Fe1-C39-H39 & 125.6 \\
\hline C39-C40-C41 & $108.30(19)$ & C39-C40-Fe1 & $70.21(12)$ \\
\hline C41-C40-Fe1 & $69.02(11)$ & C39-C40-H40 & 125.8 \\
\hline C41-C40-H40 & 125.8 & Fe1-C40-H40 & 125.8 \\
\hline C40-C41-C37 & 108.09(19) & C40-C41-Fe1 & $70.04(12)$ \\
\hline C37-C41-Fe1 & $69.87(11)$ & C40-C41-H41 & 126.0 \\
\hline C37-C41-H41 & 126.0 & Fe1-C41-H41 & 126.0 \\
\hline $\mathrm{C} 43-\mathrm{C} 42-\mathrm{C} 46$ & $107.59(17)$ & $\mathrm{C} 43-\mathrm{C} 42-\mathrm{P} 2$ & $122.19(14)$ \\
\hline C46-C42-P2 & $129.84(16)$ & C43-C42-Fe1 & $69.56(11)$ \\
\hline C46-C42-Fe1 & $70.10(11)$ & P2-C42-Fe1 & $120.18(10)$ \\
\hline C42-C43-C44 & $107.79(17)$ & C42-C43-Fe1 & $69.29(11)$ \\
\hline $\mathrm{C} 44-\mathrm{C} 43-\mathrm{Fe} 1$ & $70.30(12)$ & C42-C43-H43 & 126.1 \\
\hline C44-C43-H43 & 126.1 & Fe1-C43-H43 & 126.1 \\
\hline C45-C44-C43 & 108.29(19) & C45-C44-Fe1 & $70.15(12)$ \\
\hline C43-C44-Fe1 & $68.80(11)$ & C45-C44-H44 & 125.8 \\
\hline C43-C44-H44 & 125.8 & Fe1-C44-H44 & 125.8 \\
\hline C44-C45-C46 & $108.36(18)$ & C44-C45-Fe1 & $69.55(12)$ \\
\hline C46-C45-Fe1 & $69.38(12)$ & C44-C45-H45 & 125.8 \\
\hline C46-C45-H45 & 125.8 & Fe1-C45-H45 & 125.8 \\
\hline C45-C46-C42 & 107.97(18) & $\mathrm{C} 45-\mathrm{C} 46-\mathrm{Fe} 1$ & $70.27(12)$ \\
\hline C42-C46-Fe1 & $68.64(11)$ & C45-C46-H46 & 126.0 \\
\hline C42-C46-H46 & 126.0 & Fe1-C46-H46 & 126.0 \\
\hline
\end{tabular}

Table S6f. Torsion angles $\left({ }^{\circ}\right)$ for 8 e.

P1-Ni1-O1-C1 -18.06(15) P2-Ni1-O1-C1 177.41(10) 


$\begin{array}{llll}\text { Ni1-O1-C1-C2 } & 177.0(3) & \text { P1-Ni1-C1-O1 } & 170.68(8) \\ \text { P2-Ni1-C1-O1 } & -4.22(16) & \text { O1-Ni1-C1-C2 } & -175.1(4) \\ \text { P1-Ni1-C1-C2 } & -4.4(4) & \text { P2-Ni1-C1-C2 } & -179.3(3) \\ \text { O1-C1-C2-C7 } & -7.1(4) & \text { Ni1-C1-C2-C7 } & 166.2(3) \\ \text { O1-C1-C2-C3 } & 171.2(2) & \text { Ni1-C1-C2-C3 } & -15.4(5) \\ \text { C1-C2-C3-C6 } & 122.5(2) & \text { C7-C2-C3-C6 } & -59.2(3) \\ \text { C1-C2-C3-C4 } & -107.0(2) & \text { C7-C2-C3-C4 } & 71.3(3) \\ \text { C6-C3-C4-C5 } & -56.8(4) & \text { C2-C3-C4-C5 } & 170.4(4) \\ \text { C2-C3-C6-C5A } & 176.9(3) & \text { C4-C3-C6-C5A } & 45.5(4) \\ \text { C1-C2-C7-C8 } & -10.5(3) & \text { C3-C2-C7-C8 } & 171.3(2) \\ \text { C1-C2-C7-C12 } & 171.3(2) & \text { C3-C2-C7-C12 } & -7.0(3) \\ \text { C12-C7-C8-C9 } & -0.4(4) & \text { C2-C7-C8-C9 } & -178.8(2) \\ \text { C7-C8-C9-C10 } & 0.4(5) & \text { C8-C9-C10-C11 } & 0.2(5) \\ \text { C9-C10-C11-C12 } & -0.6(4) & \text { C10-C11-C12-C7 } & 0.6(4) \\ \text { C8-C7-C12-C11 } & 0.0(4) & \text { C2-C7-C12-C11 } & 178.3(2) \\ \text { C37-P1-C13-C18 } & -90.84(19) & \text { C19-P1-C13-C18 } & 14.8(2) \\ \text { Ni1-P1-C13-C18 } & 141.73(17) & \text { C37-P1-C13-C14 } & 86.54(17) \\ \text { C19-P1-C13-C14 } & -167.82(16) & \text { Ni1-P1-C13-C14 } & -40.89(17) \\ \text { C18-C13-C14-C15 } & 0.8(3) & \text { P1-C13-C14-C15 } & -176.74(17) \\ \text { C13-C14-C15-C16 } & -1.5(3) & \text { C14-C15-C16-C17 } & 0.7(4) \\ \text { C15-C16-C17-C18 } & 0.7(4) & \text { C14-C13-C18-C17 } & 0.6(3) \\ \text { P1-C13-C18-C17 } & 177.95(19) & \text { C16-C17-C18-C13 } & -1.4(4) \\ \text { C37-P1-C19-C20 } & 27.00(19) & \text { C13-P1-C19-C20 } & -76.41(18) \\ \text { Ni1-P1-C19-C20 } & 157.16(15) & \text { C37-P1-C19-C24 } & -149.94(17) \\ \text { C13-P1-C19-C24 } & 106.66(17) & \text { Ni1-P1-C19-C24 } & -19.78(19) \\ \text { C24-C19-C20-C21 } & -0.8(3) & \text { P1-C19-C20-C21 } & -177.70(17) \\ \text { C23-C30 } & -3.9(2) & \text { C31-P2-C25-C30 } & -113.3(2) \\ \text { C23-C24 } & -0.6(4) & \text { C22-C23-C24-C19 } & 0.7(4) \\ \text { C.9(4) } & \text { C20-C21-C22-C23 } & -0.2(4) \\ \text { C23 } & & & \end{array}$




$\begin{array}{llll}\text { Ni1-P2-C25-C30 } & 120.3(2) & \text { C42-P2-C25-C26 } & 176.48(16) \\ \text { C31-P2-C25-C26 } & 67.04(18) & \text { Ni1-P2-C25-C26 } & -59.39(18) \\ \text { C30-C25-C26-C27 } & 0.2(3) & \text { P2-C25-C26-C27 } & 179.90(18) \\ \text { C25-C26-C27-C28 } & -1.2(4) & \text { C26-C27-C28-C29 } & 1.0(4) \\ \text { C27-C28-C29-C30 } & 0.3(5) & \text { C26-C25-C30-C29 } & 1.0(4) \\ \text { P2-C25-C30-C29 } & -178.7(2) & \text { C28-C29-C30-C25 } & -1.3(5) \\ \text { C42-P2-C31-C32 } & 117.35(16) & \text { C25-P2-C31-C32 } & -134.05(17) \\ \text { Ni1-P2-C31-C32 } & -8.82(18) & \text { C42-P2-C31-C36 } & -62.62(18) \\ \text { C25-P2-C31-C36 } & 45.97(19) & \text { Ni1-P2-C31-C36 } & 171.21(15) \\ \text { C36-C31-C32-C33 } & 2.4(3) & \text { P2-C31-C32-C33 } & -177.61(17) \\ \text { C31-C32-C33-C34 } & -0.9(3) & \text { C32-C33-C34-C35 } & -1.0(4) \\ \text { C33-C34-C35-C36 } & 1.5(4) & \text { C34-C35-C36-C31 } & 0.0(3) \\ \text { C32-C31-C36-C35 } & -1.9(3) & \text { P2-C31-C36-C35 } & 178.09(17) \\ \text { Ni1-P2-C42-C43 } & -30.05(18) & \text { C31-P2-C42-C46 } & 13.9(2) \\ \text { C19-P1-C37-C41 } & 52.21(19) & \text { C13-P1-C37-C41 } & 160.84(18) \\ \text { Ni1-P1-C37-C41 } & -75.26(18) & \text { C19-P1-C37-C38 } & -129.62(18) \\ \text { C31-C37-C41-C40 } & 178.93(15) & \text { Fe1-C37-C41-C40 } & 59.86(15) \\ \text { C13-P1-C37-C38 } & -20.99(19) & \text { Ni1-P1-C37-C38 } & 102.91(17) \\ \text { C19-P1-C37-Fe1 } & 140.69(12) & \text { C13-P1-C37-Fe1 } & -110.68(13) \\ \text { Ni1-P1-C37-Fe1 } & 13.22(15) & \text { C41-C37-C38-C39 } & -0.4(2) \\ \text { P1-C37-C38-C39 } & -178.89(15) & \text { Fe1-C37-C38-C39 } & -59.42(14) \\ \text { C41-C37-C38-Fe1 } & 58.99(14) & \text { P1-C37-C38-Fe1 } & -119.47(16) \\ \text { C37-C38-C39-C40 } & 0.2(2) & \text { Fe1-C38-C39-C40 } & -58.86(16) \\ \text { C37-C38-C39-Fe1 } & 59.10(14) & \text { C38-C39-C40-C41 } & 0.1(3) \\ \text { Fe1-C39-C40-C41 } & -58.69(15) & \text { C38-C39-C40-Fe1 } & 58.75(15) \\ \text { C39-C41-C37 } & -0.3(2) & \text { Fe1-C40-C41-C37 } & -59.75(14) \\ \text { C39-C41-Fe1 } & 59.43(16) & \text { C38-C37-C41-C40 } & 0.5(2) \\ \text { P1-C37-C41-Fe1 } & 119.07(15) \\ \text { Ni1-P2-C42-C46 } & 141.98(17)\end{array}$




$\begin{array}{lllll}\text { C31-P2-C42-Fe1 } & -74.54(12) & \text { C25-P2-C42-Fe1 } & 178.54(10) \\ \text { Ni1-P2-C42-Fe1 } & 53.56(12) & \text { C46-C42-C43-C44 } & 0.0(2) \\ \text { P2-C42-C43-C44 } & 173.55(14) & \text { Fe1-C42-C43-C44 } & 60.01(14) \\ \text { C46-C42-C43-Fe1 } & -60.05(13) & \text { P2-C42-C43-Fe1 } & 113.54(14) \\ \text { C42-C43-C44-C45 } & -0.2(2) & \text { Fe1-C43-C44-C45 } & 59.21(15) \\ \text { C42-C43-C44-Fe1 } & -59.38(13) & \text { C43-C44-C45-C46 } & 0.3(2) \\ \text { Fe1-C44-C45-C46 } & 58.69(15) & \text { C43-C44-C45-Fe1 } & -58.38(14) \\ \text { C44-C45-C46-C42 } & -0.3(2) & \text { Fe1-C45-C46-C42 } & 58.46(14) \\ \text { C44-C45-C46-Fe1 } & -58.80(15) & \text { C43-C42-C46-C45 } & 0.2(2) \\ \text { P2-C42-C46-C45 } & -172.70(16) & \text { Fe1-C42-C46-C45 } & -59.47(15) \\ \text { C43-C42-C46-Fe1 } & 59.71(13) & \text { P2-C42-C46-Fe1 } & -113.23(17)\end{array}$

Table S6g. Anisotropic atomic displacement parameters $\left(\AA^{2}\right)$ for 8 e.

The anisotropic atomic displacement factor exponent takes the form: $-2 \pi^{2}$ [ $\left.\mathrm{h}^{2} \mathrm{a}^{* 2} \mathrm{U}_{11}+\ldots+2 \mathrm{~h} \mathrm{k} \mathrm{a}^{*} \mathrm{~b}^{*} \mathrm{U}_{12}\right]$

$\begin{array}{lllllll} & \mathbf{U}_{11} & \mathbf{U}_{\mathbf{2 2}} & \mathbf{U}_{\mathbf{3 3}} & \mathbf{U}_{\mathbf{2 3}} & \mathbf{U}_{\mathbf{1 3}} & \mathbf{U}_{\mathbf{1 2}} \\ \mathrm{Ni1} & 0.02006(12) & 0.02244(12) & 0.01989(12) & -0.00061(9) & 0.00753(9) & 0.00014(9) \\ \mathrm{Fe} 1 & 0.02625(15) & 0.02797(15) & 0.02139(14) & -0.00122(11) & 0.00962(11) & 0.00031(11) \\ \text { P1 } & 0.0191(2) & 0.0250(2) & 0.0235(2) & -0.00052(19) & 0.00732(19) & -0.00029(18) \\ \text { P2 } & 0.0226(2) & 0.0226(2) & 0.0238(2) & -0.00108(19) & 0.00894(19) & 0.00021(19) \\ \text { O1 } & 0.0249(7) & 0.0285(7) & 0.0245(7) & -0.0027(5) & 0.0127(6) & -0.0006(5) \\ \text { C1 } & 0.0258(10) & 0.0251(9) & 0.0223(9) & 0.0018(7) & 0.0095(8) & 0.0025(8) \\ \text { C2 } & 0.0366(11) & 0.0274(10) & 0.0250(10) & -0.0007(8) & 0.0119(9) & 0.0000(8) \\ \text { C3 } & 0.0454(13) & 0.0412(13) & 0.0390(12) & -0.0145(10) & 0.0183(11) & -0.0116(11) \\ \text { C4 } & 0.0622(19) & 0.0624(19) & 0.068(2) & -0.0162(16) & -0.0017(16) & 0.0027(15) \\ \text { C5 } & 0.045(4) & 0.094(6) & 0.148(8) & -0.042(6) & -0.044(4) & -0.008(4) \\ \text { C5A } & 0.091(5) & 0.091(5) & 0.089(5) & -0.032(4) & 0.045(4) & -0.045(4) \\ \text { C6 } & 0.075(2) & 0.0425(14) & 0.0573(17) & -0.0095(12) & 0.0317(15) & -0.0112(13) \\ \text { C7 } & 0.0439(12) & 0.0279(10) & 0.0254(10) & 0.0007(8) & 0.0147(9) & 0.0037(9) \\ \text { C8 } & 0.0418(14) & 0.0577(16) & 0.0367(13) & -0.0155(11) & 0.0115(11) & 0.0108(12)\end{array}$




\begin{tabular}{|c|c|c|c|c|c|c|}
\hline & $\mathrm{U}_{11}$ & $\mathbf{U}_{22}$ & $\mathbf{U}_{33}$ & $\mathbf{U}_{23}$ & $\mathbf{U}_{13}$ & $\mathbf{U}_{12}$ \\
\hline C9 & $0.0425(14)$ & $0.079(2)$ & $0.0492(15)$ & $-0.0174(14)$ & $0.0153(12)$ & $0.0142(14)$ \\
\hline $\mathrm{C} 10$ & $0.0574(17)$ & $0.0536(16)$ & $0.0467(14)$ & $-0.0054(12)$ & $0.0276(13)$ & $0.0136(13)$ \\
\hline $\mathrm{C} 11$ & $0.079(2)$ & $0.0414(14)$ & $0.0439(14)$ & $-0.0124(11)$ & $0.0326(14)$ & $0.0016(13)$ \\
\hline $\mathrm{C} 12$ & $0.0574(16)$ & $0.0403(13)$ & $0.0411(13)$ & $-0.0106(10)$ & $0.0264(12)$ & $0.0106(11)$ \\
\hline $\mathrm{C} 13$ & $0.0256(10)$ & $0.0262(10)$ & $0.0291(10)$ & $0.0004(8)$ & $0.0123(8)$ & $-0.0005(8)$ \\
\hline $\mathrm{C} 14$ & $0.0308(11)$ & $0.0342(11)$ & $0.0334(11)$ & $0.0038(9)$ & $0.0099(9)$ & $0.0028(9)$ \\
\hline $\mathrm{C} 15$ & $0.0480(14)$ & $0.0357(12)$ & $0.0437(13)$ & 0.0130 & $0.0204(11)$ & $0.0114(11)$ \\
\hline $\mathrm{C} 16$ & $0.0599(16)$ & $0.0287(12)$ & $0.0676(17)$ & $0.0105(11)$ & $0.0390(14)$ & $0.0018(11)$ \\
\hline $\mathrm{C} 17$ & $0.0431(14)$ & $0.0351(13)$ & $0.083(2)$ & $-0.0048(13)$ & $0.0314(14)$ & $-0.0128(11)$ \\
\hline $\mathrm{C} 18$ & $0.0276(11)$ & $0.0334(12)$ & $0.0553(15)$ & $-0.0037(10)$ & $0.0130(10)$ & $-0.0026(9)$ \\
\hline C19 & $0.0209(9)$ & $0.0257(10)$ & $0.0284(10)$ & $-0.0031(8)$ & $0.0061(8)$ & $0.0006(7)$ \\
\hline $\mathrm{C} 20$ & $0.0240(10)$ & $0.0355(11)$ & $0.0366(11)$ & $-0.0025(9)$ & $0.0102(9)$ & $-0.0006(8)$ \\
\hline $\mathrm{C} 21$ & $0.0218(11)$ & $0.0520(14)$ & $0.0499(14)$ & $-0.0062(11)$ & $0.0102(10)$ & $0.0056(10)$ \\
\hline $\mathrm{C} 22$ & $0.0275(12)$ & $0.0665(17)$ & $0.0431(14)$ & $-0.0003(12)$ & $-0.0003(10)$ & $0.0143(11)$ \\
\hline $\mathrm{C} 23$ & $0.0353(13)$ & $0.0713(18)$ & $0.0297(12)$ & $0.0057(12)$ & $0.0031(10)$ & $0.0114(12)$ \\
\hline $\mathrm{C} 24$ & $0.0242(10)$ & $0.0489(13)$ & $0.0274(10)$ & $-0.0004(9)$ & $0.0069(8)$ & $0.0046(9)$ \\
\hline $\mathrm{C} 25$ & $0.0261(10)$ & $0.0221(10)$ & $0.0371(11)$ & $-0.0020(8)$ & $0.0137(9)$ & $0.0006(8)$ \\
\hline $\mathrm{C} 26$ & $0.0392(12)$ & $0.0399(13)$ & $0.0395(12)$ & $-0.0048(10)$ & $0.0204(10)$ & $-0.0064(10)$ \\
\hline $\mathrm{C} 27$ & $0.0490(15)$ & $0.0414(13)$ & $0.0580(16)$ & $-0.0028(11)$ & $0.0345(13)$ & $-0.0083(11)$ \\
\hline $\mathrm{C} 28$ & $0.0349(13)$ & $0.0457(15)$ & $0.088(2)$ & $0.0064(14)$ & $0.0344(14)$ & $-0.0014(11)$ \\
\hline $\mathrm{C} 29$ & $0.0272(13)$ & $0.088(2)$ & 0.07 & 0.0 & 0.002 & -0.0087 \\
\hline $\mathrm{C} 30$ & $0.0295(12)$ & $0.0723(19)$ & $0.0503(15)$ & $0.0215(14)$ & $0.0053(11)$ & $-0.0077(12)$ \\
\hline $\mathrm{C} 31$ & $0.0309(10)$ & $0.0246(10)$ & $0.0299(10)$ & $0.0028(8)$ & $0.0154(8)$ & $0.0024(8)$ \\
\hline $\mathrm{C} 32$ & $0.0346(12)$ & $0.0309(11)$ & $0.0405(12)$ & $0.0068(9)$ & $0.0120(10)$ & $0.0018(9)$ \\
\hline $\mathrm{C} 33$ & $0.0380(13)$ & $0.0473(15)$ & $0.0521(15)$ & $0.0186(12)$ & $0.0142(11)$ & $0.0117(11)$ \\
\hline C34 & $0.0576(16)$ & $0.0391(14)$ & $0.0548(15)$ & $0.0171(12)$ & $0.0282(13)$ & $0.0226(12)$ \\
\hline C35 & $0.0628(16)$ & $0.0255(11)$ & $0.0477(14)$ & 0.0014 & $0.0258(13)$ & $0.0073(11)$ \\
\hline $\mathrm{C} 36$ & $0.0392(12)$ & $0.0277(11)$ & $0.0376(12)$ & $-0.0009(9)$ & $0.0160(10)$ & $0.0008(9)$ \\
\hline $\mathrm{C} 37$ & $0.0211(9)$ & $0.0329(11)$ & $0.0254(10)$ & $-0.0011(8)$ & $0.0084(8)$ & $-0.0017(8)$ \\
\hline
\end{tabular}




$\begin{array}{lllllll} & \mathbf{U}_{11} & \mathbf{U}_{22} & \mathbf{U}_{33} & \mathbf{U}_{23} & \mathbf{U}_{13} & \mathbf{U}_{12} \\ \mathrm{C} 38 & 0.0301(11) & 0.0361(11) & 0.0291(10) & 0.0022(9) & 0.0115(9) & -0.0066(9) \\ \text { C39 } & 0.0359(12) & 0.0521(14) & 0.0282(11) & -0.0006(10) & 0.0177(9) & -0.0084(10) \\ \text { C40 } & 0.0307(11) & 0.0493(14) & 0.0351(12) & -0.0045(10) & 0.0184(9) & 0.0030(10) \\ \text { C41 } & 0.0254(10) & 0.0378(11) & 0.0308(10) & -0.0011(9) & 0.0109(8) & 0.0054(9) \\ \text { C42 } & 0.0235(9) & 0.0251(9) & 0.0228(9) & -0.0048(7) & 0.0086(7) & -0.0040(7) \\ \text { C43 } & 0.0238(9) & 0.0286(10) & 0.0271(10) & 0.0001(8) & 0.0074(8) & 0.0022(8) \\ \text { C44 } & 0.0295(11) & 0.0398(12) & 0.0246(10) & 0.0024(9) & 0.0038(8) & 0.0034(9) \\ \text { C45 } & 0.0357(12) & 0.0427(12) & 0.0229(10) & -0.0069(9) & 0.0075(9) & -0.0027(9) \\ \text { C46 } & 0.0319(11) & 0.0296(10) & 0.0276(10) & -0.0078(8) & 0.0096(9) & -0.0034(8)\end{array}$

Table S6h. Hydrogen atomic coordinates and isotropic atomic displacement parameters $\left(\AA^{2}\right)$ for $8 \mathrm{e}$.

$\begin{array}{lllll} & \mathbf{x} / \mathbf{a} & \mathbf{y} / \mathbf{b} & \mathbf{z} / \mathbf{c} & \mathbf{U}(\mathbf{e q}) \\ \text { H3 } & 0.7707 & 0.4317 & 0.6894 & 0.049 \\ \text { H4A } & 0.7966 & 0.3827 & 0.8088 & 0.082 \\ \text { H4B } & 0.7647 & 0.4560 & 0.8397 & 0.082 \\ \text { H4C } & 0.7920 & 0.3835 & 0.8089 & 0.103 \\ \text { H4D } & 0.8918 & 0.4447 & 0.8110 & 0.103 \\ \text { H4E } & 0.7675 & 0.4581 & 0.8389 & 0.103 \\ \text { H5A } & 0.9926 & 0.4434 & 0.8653 & 0.166 \\ \text { H5B } & 0.9760 & 0.4332 & 0.7776 & 0.166 \\ \text { H5C } & 0.9442 & 0.5062 & 0.8083 & 0.166 \\ \text { H5A1 } & 0.8950 & 0.6049 & 0.7359 & 0.13 \\ \text { H5A2 } & 0.9182 & 0.5453 & 0.7980 & 0.13 \\ \text { H5A3 } & 0.9412 & 0.5300 & 0.7182 & 0.13 \\ \text { H6A } & 0.8261 & 0.5445 & 0.7277 & 0.083 \\ \text { H6B } & 0.7013 & 0.5410 & 0.6566 & 0.083 \\ \text { H6C } & 0.6865 & 0.5561 & 0.7384 & 0.083 \\ \text { H6D } & 0.6816 & 0.5564 & 0.7358 & 0.067\end{array}$




\begin{tabular}{|c|c|c|c|c|}
\hline & $\mathbf{x} / \mathbf{a}$ & $\mathbf{y} / \mathbf{b}$ & $\mathbf{z} / \mathbf{c}$ & $\mathrm{U}(\mathbf{e q})$ \\
\hline H6E & 0.7046 & 0.5410 & 0.6559 & 0.067 \\
\hline H8 & 0.3398 & 0.3964 & 0.6595 & 0.054 \\
\hline H9 & 0.1765 & 0.4353 & 0.7063 & 0.068 \\
\hline H10 & 0.2188 & 0.5128 & 0.8055 & 0.06 \\
\hline H11 & 0.4271 & 0.5514 & 0.8569 & 0.062 \\
\hline H12 & 0.5935 & 0.5119 & 0.8121 & 0.052 \\
\hline H14 & 0.5949 & 0.4578 & 0.4929 & 0.039 \\
\hline H15 & 0.6011 & 0.5770 & 0.4779 & 0.049 \\
\hline H16 & 0.7929 & 0.6363 & 0.5224 & 0.057 \\
\hline H17 & 0.9807 & 0.5762 & 0.5798 & 0.061 \\
\hline H18 & 0.9778 & 0.4563 & 0.5909 & 0.046 \\
\hline $\mathrm{H} 20$ & 1.0369 & 0.3317 & 0.5408 & 0.038 \\
\hline $\mathrm{H} 21$ & 1.2248 & 0.2892 & 0.6245 & 0.05 \\
\hline $\mathrm{H} 22$ & 1.2238 & 0.2509 & 0.7428 & 0.057 \\
\hline $\mathrm{H} 23$ & 1.0352 & 0.2555 & 0.7788 & 0.056 \\
\hline $\mathrm{H} 24$ & 0.8476 & 0.3004 & 0.6965 & 0.04 \\
\hline $\mathrm{H} 26$ & 0.4373 & 0.1710 & 0.6123 & 0.045 \\
\hline $\mathrm{H} 27$ & 0.2433 & 0.1267 & 0.6219 & 0.055 \\
\hline $\mathrm{H} 28$ & 0.0634 & 0.1273 & 0.5187 & 0.063 \\
\hline H29 & 0.0772 & 0.1690 & 0.4047 & 0.081 \\
\hline H30 & 0.2729 & 0.2100 & 0.3927 & 0.062 \\
\hline H32 & 0.7780 & 0.1955 & 0.5912 & 0.042 \\
\hline H33 & 0.9136 & 0.0994 & 0.6105 & 0.055 \\
\hline H34 & 0.8435 & -0.0027 & 0.5452 & 0.058 \\
\hline H35 & 0.6376 & -0.0099 & 0.4637 & 0.052 \\
\hline H36 & 0.5026 & 0.0858 & 0.4426 & 0.041 \\
\hline H38 & 0.7444 & 0.4247 & 0.3898 & 0.037 \\
\hline H39 & 0.7856 & 0.3549 & 0.2797 & 0.044 \\
\hline $\mathrm{H} 40$ & 0.8477 & 0.2308 & 0.3248 & 0.044 \\
\hline
\end{tabular}




$\begin{array}{lllll} & \mathbf{x} / \mathbf{a} & \mathbf{y} / \mathbf{b} & \mathbf{z} / \mathbf{c} & \mathbf{U}(\mathbf{e q}) \\ \mathrm{H} 41 & 0.8454 & 0.2213 & 0.4638 & 0.037 \\ \mathrm{H} 43 & 0.4499 & 0.3577 & 0.4003 & 0.032 \\ \mathrm{H} 44 & 0.4551 & 0.3599 & 0.2613 & 0.039 \\ \mathrm{H} 45 & 0.5190 & 0.2383 & 0.2271 & 0.041 \\ \mathrm{H} 46 & 0.5530 & 0.1597 & 0.3438 & 0.035\end{array}$


Table S7a. Sample and crystal data for $8 \mathrm{ca}$.

\begin{tabular}{|c|c|c|}
\hline Identification code & \multicolumn{2}{|l|}{$8 \mathrm{ca}$} \\
\hline Chemical formula & \multicolumn{2}{|l|}{$\mathrm{C}_{46} \mathrm{H}_{41} \mathrm{FFeNiOP}{ }_{2}$} \\
\hline Formula weight & \multicolumn{2}{|l|}{$805.29 \mathrm{~g} / \mathrm{mol}$} \\
\hline Temperature & \multicolumn{2}{|l|}{$150(1) \mathrm{K}$} \\
\hline Wavelength & \multicolumn{2}{|l|}{$0.71073 \AA$} \\
\hline Crystal size & \multicolumn{2}{|c|}{$0.168 \times 0.196 \times 0.420 \mathrm{~mm}$} \\
\hline Crystal habit & \multicolumn{2}{|c|}{ clear intense orange prism } \\
\hline Crystal system & \multicolumn{2}{|l|}{ monoclinic } \\
\hline Space group & \multicolumn{2}{|l|}{ P $121 / \mathrm{c} 1$} \\
\hline \multirow[t]{3}{*}{ Unit cell dimensions } & $\mathrm{a}=10.8518(3) \AA$ & $\alpha=90^{\circ}$ \\
\hline & $\mathrm{b}=20.0649(6) \AA$ & $\beta=107.1420(10)^{\circ}$ \\
\hline & $\mathrm{c}=18.0547(5) \AA$ & $\gamma=90^{\circ}$ \\
\hline Volume & \multicolumn{2}{|l|}{$3756.60(19) \AA^{3}$} \\
\hline $\mathbf{Z}$ & \multicolumn{2}{|l|}{4} \\
\hline Density (calculated) & \multicolumn{2}{|l|}{$1.424 \mathrm{~g} / \mathrm{cm}^{3}$} \\
\hline Absorption coefficient & \multicolumn{2}{|l|}{$1.014 \mathrm{~mm}^{-1}$} \\
\hline $\mathbf{F}(000)$ & \multicolumn{2}{|l|}{1672} \\
\hline
\end{tabular}

Table S7b. Data collection and structure refinement for $8 \mathrm{ca}$.

Theta range for data collection $\quad 2.03$ to $27.88^{\circ}$

Index ranges $-14<=\mathrm{h}<=12,-26<=\mathrm{k}<=26,-23<=\mathrm{l}<=23$

Reflections collected $\quad 99967$

Independent reflections $\quad 8959[\mathrm{R}(\mathrm{int})=0.0351]$

Coverage of independent reflections $100.0 \%$

Absorption correction multi-scan

Max. and min. transmission $\quad 0.8480$ and 0.6760

Structure solution technique direct methods

Structure solution program $\quad$ SHELXT-2014/9 (Sheldrick, 2014) 


\begin{tabular}{|c|c|}
\hline Refinement method & Full-matrix least-squares on $\mathrm{F}^{2}$ \\
\hline Refinement program & SHELXL-2014/6 (Sheldrick, 2014) \\
\hline Function minimized & $\Sigma \mathrm{w}\left(\mathrm{F}_{\mathrm{o}}^{2}-\mathrm{F}_{\mathrm{c}}^{2}\right)^{2}$ \\
\hline Data / restraints / parameters & $8959 / 0 / 471$ \\
\hline Goodness-of-fit on $F^{2}$ & 1.054 \\
\hline$\Delta / \sigma_{\max }$ & 0.001 \\
\hline \multirow[t]{2}{*}{ Final $R$ indices } & 7503 data; $\mathrm{I}>2 \sigma(\mathrm{I}) \mathrm{R} 1=0.0302, \mathrm{wR} 2=0.0738$ \\
\hline & $\mathrm{R} 1=0.0403, \mathrm{wR} 2=0.0785$ \\
\hline \multirow{2}{*}{ Weighting scheme } & $\mathrm{w}=1 /\left[\sigma^{2}\left(\mathrm{~F}_{\mathrm{o}}^{2}\right)+(0.0372 \mathrm{P})^{2}+2.2484 \mathrm{P}\right]$ \\
\hline & where $\mathrm{P}=\left(\mathrm{F}_{\mathrm{o}}^{2}+2 \mathrm{~F}_{\mathrm{c}}^{2}\right) / 3$ \\
\hline Largest diff. peak and hole & 0.378 and $-0.380 \mathrm{e}^{-3}$ \\
\hline R.M.S. deviation from mean & $0.061 \mathrm{e} \AA^{-3}$ \\
\hline
\end{tabular}

Table S7c. Atomic coordinates and equivalent isotropic atomic displacement parameters $\left(\AA^{2}\right)$ for

8ca.

$\mathrm{U}(\mathrm{eq})$ is defined as one third of the trace of the orthogonalized $\mathrm{U}_{\mathrm{ij}}$ tensor.

$\begin{array}{lllll} & \mathbf{x} / \mathbf{a} & \mathbf{y} / \mathbf{b} & \mathbf{z} / \mathbf{c} & \mathbf{U}(\mathbf{e q}) \\ \mathrm{Ni1} & 0.59163(2) & 0.31989(2) & 0.56505(2) & 0.01609(6) \\ \mathrm{Fe} 1 & 0.62965(2) & 0.30262(2) & 0.34926(2) & 0.01926(6) \\ \mathrm{P} 1 & 0.76199(4) & 0.35376(2) & 0.53816(2) & 0.01670(9) \\ \mathrm{P} 2 & 0.52022(4) & 0.23414(2) & 0.48545(2) & 0.01777(9) \\ \mathrm{F} 1 & 0.14771(12) & 0.48042(8) & 0.81507(8) & 0.0560(4) \\ \text { O1 } & 0.46095(11) & 0.32663(6) & 0.61131(7) & 0.0204(2) \\ \mathrm{C} 1 & 0.54314(15) & 0.37398(8) & 0.63727(9) & 0.0197(3) \\ \mathrm{C} 2 & 0.54962(16) & 0.42647(8) & 0.68525(10) & 0.0211(3) \\ \mathrm{C} 3 & 0.66410(17) & 0.47301(9) & 0.70269(10) & 0.0260(4) \\ \mathrm{C} 4 & 0.75545(18) & 0.47271(10) & 0.78608(12) & 0.0315(4) \\ \mathrm{C} 5 & 0.8197(2) & 0.40534(13) & 0.80714(16) & 0.0544(7)\end{array}$




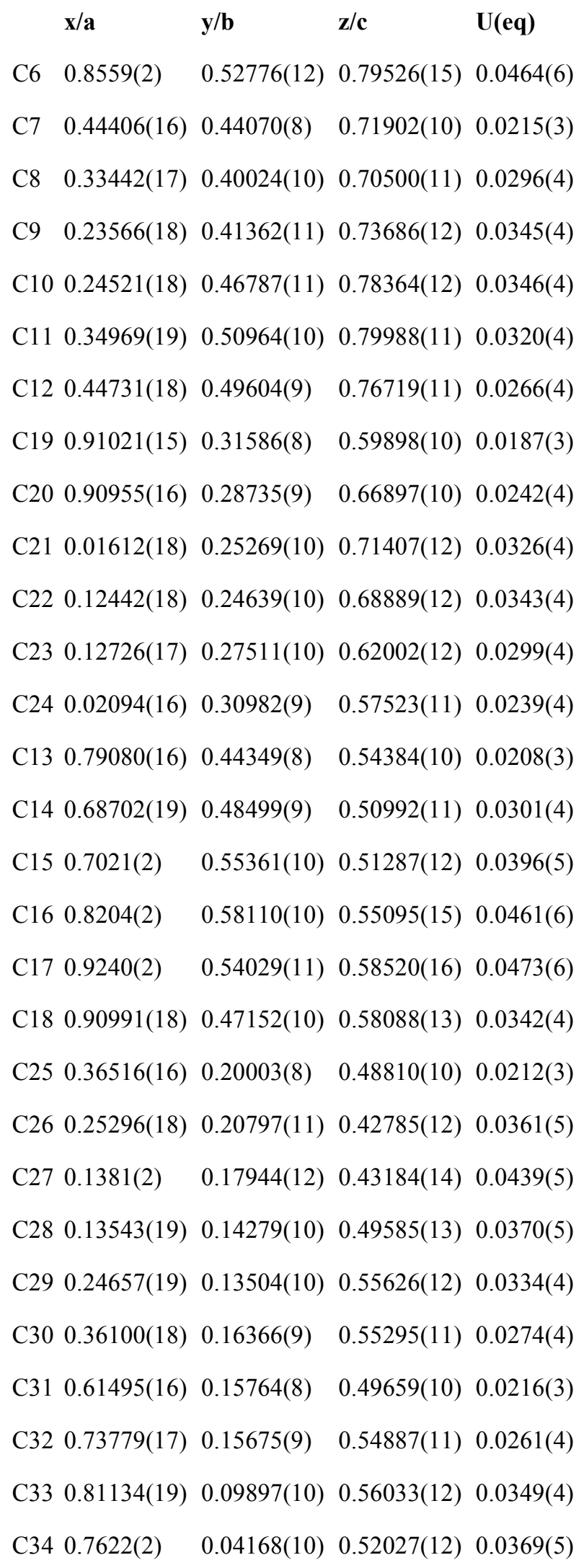




\begin{tabular}{lllll}
\multicolumn{1}{c}{$\mathbf{x} / \mathbf{a}$} & $\mathbf{y} / \mathbf{b}$ & $\mathbf{z} / \mathbf{c}$ & $\mathbf{U}(\mathbf{e q})$ \\
$\mathrm{C} 35$ & $0.6392(2)$ & $0.04190(9)$ & $0.46854(12)$ & $0.0333(4)$ \\
$\mathrm{C} 36$ & $0.56542(18)$ & $0.09929(9)$ & $0.45645(11)$ & $0.0272(4)$ \\
$\mathrm{C} 37$ & $0.77483(15)$ & $0.33635(8)$ & $0.44169(9)$ & $0.0192(3)$ \\
$\mathrm{C} 38$ & $0.75446(16)$ & $0.38169(9)$ & $0.37786(10)$ & $0.0233(3)$ \\
$\mathrm{C} 39$ & $0.77477(17)$ & $0.34609(10)$ & $0.31423(10)$ & $0.0272(4)$ \\
$\mathrm{C} 40$ & $0.80684(17)$ & $0.27952(10)$ & $0.33730(11)$ & $0.0274(4)$ \\
$\mathrm{C} 41$ & $0.80684(16)$ & $0.27269(9)$ & $0.41582(10)$ & $0.0230(3)$ \\
$\mathrm{C} 42$ & $0.49004(15)$ & $0.25897(8)$ & $0.38585(10)$ & $0.0197(3)$ \\
$\mathrm{C} 43$ & $0.45347(15)$ & $0.32566(8)$ & $0.35989(10)$ & $0.0215(3)$ \\
$\mathrm{C} 44$ & $0.44923(16)$ & $0.32928(9)$ & $0.28051(10)$ & $0.0255(4)$ \\
$\mathrm{C} 45$ & $0.48352(17)$ & $0.26623(9)$ & $0.25740(10)$ & $0.0272(4)$ \\
$\mathrm{C} 46$ & $0.50821(17)$ & $0.22245(9)$ & $0.32167(10)$ & $0.0241(4)$
\end{tabular}

Table S7d. Bond lengths ( $($ ) for $8 \mathrm{ca}$.

$\begin{array}{llll}\text { Ni1-O1 } & 1.8507(11) & \text { Ni1-C1 } & 1.8867(16) \\ \text { Ni1-P1 } & 2.1552(4) & \text { Ni1-P2 } & 2.2293(5) \\ \text { Fe1-C42 } & 2.0221(16) & \text { Fe1-C43 } & 2.0303(17) \\ \text { Fe1-C41 } & 2.0347(17) & \text { Fe1-C37 } & 2.0434(16) \\ \text { Fe1-C46 } & 2.0458(17) & \text { Fe1-C40 } & 2.0502(18) \\ \text { Fe1-C38 } & 2.0509(17) & \text { Fe1-C44 } & 2.0561(17) \\ \text { Fe1-C39 } & 2.0563(17) & \text { Fe1-C45 } & 2.0611(17) \\ \text { P1-C37 } & 1.8216(17) & \text { P1-C19 } & 1.8249(16) \\ \text { P1-C13 } & 1.8249(17) & \text { P2-C42 } & 1.8005(17) \\ \text { P2-C31 } & 1.8252(17) & \text { P2-C25 } & 1.8302(17) \\ \text { F1-C10 } & 1.363(2) & \text { O1-C1 } & 1.293(2) \\ \text { C1-C2 } & 1.352(2) & \text { C2-C7 } & 1.475(2) \\ \text { C2-C3 } & 1.511(2) & \text { C3-C4 } & 1.539(3) \\ \text { C3-H3A } & 0.99 & \text { C3-H3B } & 0.99\end{array}$




\begin{tabular}{|c|c|c|c|}
\hline $\mathrm{C} 4-\mathrm{C} 5$ & $1.517(3)$ & C4-C6 & $1.526(3)$ \\
\hline C4-H4 & 1.0 & C5-H5A & 0.98 \\
\hline C5-H5B & 0.98 & C5-H5C & 0.98 \\
\hline C6-H6A & 0.98 & C6-H6B & 0.98 \\
\hline C6-H6C & 0.98 & $\mathrm{C} 7-\mathrm{C} 8$ & $1.400(2)$ \\
\hline $\mathrm{C} 7-\mathrm{C} 12$ & $1.405(2)$ & C8-C9 & $1.384(3)$ \\
\hline C8-H8 & 0.95 & C9-C10 & $1.363(3)$ \\
\hline C9-H9 & 0.95 & $\mathrm{C} 10-\mathrm{C} 11$ & $1.370(3)$ \\
\hline $\mathrm{C} 11-\mathrm{C} 12$ & $1.383(3)$ & C11-H11 & 0.95 \\
\hline $\mathrm{C} 12-\mathrm{H} 12$ & 0.95 & C19-C20 & $1.389(2)$ \\
\hline C19-C24 & $1.395(2)$ & $\mathrm{C} 20-\mathrm{C} 21$ & $1.389(3)$ \\
\hline $\mathrm{C} 20-\mathrm{H} 20$ & 0.95 & $\mathrm{C} 21-\mathrm{C} 22$ & $1.385(3)$ \\
\hline $\mathrm{C} 21-\mathrm{H} 21$ & 0.95 & $\mathrm{C} 22-\mathrm{C} 23$ & $1.379(3)$ \\
\hline $\mathrm{C} 22-\mathrm{H} 22$ & 0.95 & $\mathrm{C} 23-\mathrm{C} 24$ & $1.386(3)$ \\
\hline $\mathrm{C} 23-\mathrm{H} 23$ & 0.95 & C24-H24 & 0.95 \\
\hline $\mathrm{C} 13-\mathrm{C} 18$ & $1.387(2)$ & C13-C14 & $1.389(2)$ \\
\hline C14-C15 & $1.386(3)$ & C14-H14 & 0.95 \\
\hline $\mathrm{C} 15-\mathrm{C} 16$ & $1.380(3)$ & $\mathrm{C} 15-\mathrm{H} 15$ & 0.95 \\
\hline $\mathrm{C} 16-\mathrm{C} 17$ & $1.381(3)$ & C16-H16 & 0.95 \\
\hline $\mathrm{C} 17-\mathrm{C} 18$ & $1.388(3)$ & C17-H17 & 0.95 \\
\hline C18-H18 & 0.95 & $\mathrm{C} 25-\mathrm{C} 26$ & $1.382(3)$ \\
\hline $\mathrm{C} 25-\mathrm{C} 30$ & $1.392(3)$ & $\mathrm{C} 26-\mathrm{C} 27$ & $1.393(3)$ \\
\hline C26-H26 & 0.95 & $\mathrm{C} 27-\mathrm{C} 28$ & $1.377(3)$ \\
\hline $\mathrm{C} 27-\mathrm{H} 27$ & 0.95 & C28-C29 & $1.376(3)$ \\
\hline C28-H28 & 0.95 & C29-C30 & $1.385(3)$ \\
\hline C29-H29 & 0.95 & C30-H30 & 0.95 \\
\hline C31-C32 & $1.388(2)$ & C31-C36 & $1.398(2)$ \\
\hline C32-C33 & $1.388(3)$ & $\mathrm{C} 32-\mathrm{H} 32$ & 0.95 \\
\hline C33-C34 & $1.379(3)$ & C33-H33 & 0.95 \\
\hline C34-C35 & $1.385(3)$ & C34-H34 & 0.95 \\
\hline
\end{tabular}




$\begin{array}{llll}\text { C35-C36 } & 1.383(3) & \mathrm{C} 35-\mathrm{H} 35 & 0.95 \\ \mathrm{C} 36-\mathrm{H} 36 & 0.95 & \mathrm{C} 37-\mathrm{C} 38 & 1.433(2) \\ \mathrm{C} 37-\mathrm{C} 41 & 1.437(2) & \mathrm{C} 38-\mathrm{C} 39 & 1.424(2) \\ \mathrm{C} 38-\mathrm{H} 38 & 1.0 & \mathrm{C} 39-\mathrm{C} 40 & 1.412(3) \\ \mathrm{C} 39-\mathrm{H} 39 & 1.0 & \mathrm{C} 40-\mathrm{C} 41 & 1.424(2) \\ \mathrm{C} 40-\mathrm{H} 40 & 1.0 & \mathrm{C} 41-\mathrm{H} 41 & 1.0 \\ \mathrm{C} 42-\mathrm{C} 46 & 1.433(2) & \mathrm{C} 42-\mathrm{C} 43 & 1.435(2) \\ \mathrm{C} 43-\mathrm{C} 44 & 1.422(2) & \mathrm{C} 43-\mathrm{H} 43 & 1.0 \\ \mathrm{C} 44-\mathrm{C} 45 & 1.416(3) & \mathrm{C} 44-\mathrm{H} 44 & 1.0 \\ \mathrm{C} 45-\mathrm{C} 46 & 1.417(3) & \mathrm{C} 45-\mathrm{H} 45 & 1.0 \\ \mathrm{C} 46-\mathrm{H} 46 & 1.0 & & \end{array}$

Table S7e. Bond angles $\left({ }^{\circ}\right)$ for $8 \mathrm{ca}$.

$\begin{array}{llll}\text { O1-Ni1-C1 } & 40.46(6) & \text { O1-Ni1-P1 } & 154.28(4) \\ \text { C1-Ni1-P1 } & 114.00(5) & \text { O1-Ni1-P2 } & 100.39(4) \\ \text { C1-Ni1-P2 } & 140.79(5) & \text { P1-Ni1-P2 } & 104.858(17) \\ \text { C42-Fe1-C43 } & 41.47(7) & \text { C42-Fe1-C41 } & 110.36(7) \\ \text { C43-Fe1-C41 } & 139.79(7) & \text { C42-Fe1-C37 } & 110.38(7) \\ \text { C43-Fe1-C37 } & 111.61(7) & \text { C41-Fe1-C37 } & 41.27(7) \\ \text { C42-Fe1-C46 } & 41.24(7) & \text { C43-Fe1-C46 } & 69.21(7) \\ \text { C41-Fe1-C46 } & 109.91(7) & \text { C37-Fe1-C46 } & 138.21(7) \\ \text { C42-Fe1-C40 } & 138.83(7) & \text { C43-Fe1-C40 } & 179.40(7) \\ \text { C41-Fe1-C40 } & 40.81(7) & \text { C37-Fe1-C40 } & 68.87(7) \\ \text { C46-Fe1-C40 } & 110.70(7) & \text { C42-Fe1-C38 } & 139.08(7) \\ \text { C43-Fe1-C38 } & 111.74(7) & \text { C41-Fe1-C38 } & 68.93(7) \\ \text { C37-Fe1-C38 } & 40.98(7) & \text { C46-Fe1-C38 } & 178.83(7) \\ \text { C40-Fe1-C38 } & 68.35(7) & \text { C42-Fe1-C44 } & 68.79(7) \\ \text { C43-Fe1-C44 } & 40.73(7) & \text { C41-Fe1-C44 } & 177.87(7) \\ \text { C37-Fe1-C44 } & 140.77(7) & \text { C46-Fe1-C44 } & 68.11(7) \\ \text { C40-Fe1-C44 } & 138.67(7) & \text { C38-Fe1-C44 } & 113.04(7) \\ & & & \text { S128 }\end{array}$




\begin{tabular}{|c|c|c|c|}
\hline C42-Fe1-C39 & $178.67(7)$ & C43-Fe1-C39 & $139.50(7)$ \\
\hline C41-Fe1-C39 & $68.33(7)$ & C37-Fe1-C39 & $68.57(7)$ \\
\hline C46-Fe1-C39 & $139.07(7)$ & C40-Fe1-C39 & $40.22(8)$ \\
\hline C38-Fe1-C39 & $40.57(7)$ & C44-Fe1-C39 & $112.53(7)$ \\
\hline C42-Fe1-C45 & $68.69(7)$ & C43-Fe1-C45 & $68.54(7)$ \\
\hline C41-Fe1-C45 & $137.71(7)$ & $\mathrm{C} 37-\mathrm{Fe} 1-\mathrm{C} 45$ & $178.52(7)$ \\
\hline $\mathrm{C} 46-\mathrm{Fe} 1-\mathrm{C} 45$ & $40.35(7)$ & C40-Fe1-C45 & $110.99(7)$ \\
\hline C38-Fe1-C45 & $140.45(7)$ & C44-Fe1-C45 & $40.23(7)$ \\
\hline C39-Fe1-C45 & $112.34(7)$ & C37-P1-C19 & $101.14(7)$ \\
\hline C37-P1-C13 & $100.54(8)$ & C19-P1-C13 & $105.74(8)$ \\
\hline C37-P1-Ni1 & $117.86(5)$ & C19-P1-Ni1 & $113.29(6)$ \\
\hline C13-P1-Ni1 & $116.27(6)$ & C42-P2-C31 & $105.87(8)$ \\
\hline C42-P2-C25 & $103.39(8)$ & C31-P2-C25 & $100.08(8)$ \\
\hline C42-P2-Ni1 & $110.90(5)$ & C31-P2-Ni1 & $120.03(6)$ \\
\hline C25-P2-Ni1 & $114.79(6)$ & C1-O1-Ni1 & $71.26(9)$ \\
\hline $\mathrm{O} 1-\mathrm{C} 1-\mathrm{C} 2$ & $134.80(15)$ & O1-C1-Ni1 & $68.28(9)$ \\
\hline C2-C1-Ni1 & $156.69(13)$ & $\mathrm{C} 1-\mathrm{C} 2-\mathrm{C} 7$ & $121.02(15)$ \\
\hline $\mathrm{C} 1-\mathrm{C} 2-\mathrm{C} 3$ & $119.94(15)$ & $\mathrm{C} 7-\mathrm{C} 2-\mathrm{C} 3$ & $119.01(15)$ \\
\hline $\mathrm{C} 2-\mathrm{C} 3-\mathrm{C} 4$ & $117.01(15)$ & $\mathrm{C} 2-\mathrm{C} 3-\mathrm{H} 3 \mathrm{~A}$ & 108.0 \\
\hline $\mathrm{C} 4-\mathrm{C} 3-\mathrm{H} 3 \mathrm{~A}$ & 108.0 & $\mathrm{C} 2-\mathrm{C} 3-\mathrm{H} 3 \mathrm{~B}$ & 108.0 \\
\hline C4-C3-H3B & 108.0 & H3A-C3-H3B & 107.3 \\
\hline $\mathrm{C} 5-\mathrm{C} 4-\mathrm{C} 6$ & $110.93(18)$ & $\mathrm{C} 5-\mathrm{C} 4-\mathrm{C} 3$ & $111.38(17)$ \\
\hline $\mathrm{C} 6-\mathrm{C} 4-\mathrm{C} 3$ & 109.69(18) & C5-C4-H4 & 108.3 \\
\hline C6-C4-H4 & 108.3 & $\mathrm{C} 3-\mathrm{C} 4-\mathrm{H} 4$ & 108.3 \\
\hline C4-C5-H5A & 109.5 & C4-C5-H5B & 109.5 \\
\hline H5A-C5-H5B & 109.5 & C4-C5-H5C & 109.5 \\
\hline H5A-C5-H5C & 109.5 & H5B-C5-H5C & 109.5 \\
\hline C4-C6-H6A & 109.5 & C4-C6-H6B & 109.5 \\
\hline H6A-C6-H6B & 109.5 & C4-C6-H6C & 109.5 \\
\hline H6A-C6-H6C & 109.5 & H6B-C6-H6C & 109.5 \\
\hline
\end{tabular}




\begin{tabular}{llll} 
C8-C7-C12 & $115.79(16)$ & C8-C7-C2 & $122.33(15)$ \\
C12-C7-C2 & $121.87(16)$ & C9-C8-C7 & $122.23(17)$ \\
C9-C8-H8 & 118.9 & C7-C8-H8 & 118.9 \\
C10-C9-C8 & $119.06(18)$ & C10-C9-H9 & 120.5 \\
C8-C9-H9 & 120.5 & C9-C10-F1 & $118.61(18)$ \\
C9-C10-C11 & $121.87(18)$ & F1-C10-C11 & $119.52(18)$ \\
C10-C11-C12 & $118.53(18)$ & C10-C11-H11 & 120.7 \\
C12-C11-H11 & 120.7 & C11-C12-C7 & $122.52(17)$ \\
C11-C12-H12 & 118.7 & C7-C12-H12 & 118.7 \\
C20-C19-C24 & $118.67(15)$ & C20-C19-P1 & $118.63(12)$ \\
C24-C19-P1 & $122.51(13)$ & C21-C20-C19 & $120.86(16)$ \\
C21-C20-H20 & 119.6 & C19-C20-H20 & 119.6 \\
C22-C21-C20 & $119.58(18)$ & C22-C21-H21 & 120.2 \\
C20-C21-H21 & 120.2 & C23-C22-C21 & $120.29(17)$ \\
C23-C22-H22 & 119.9 & C21-C22-H22 & 119.9 \\
C22-C23-C24 & $120.01(17)$ & C22-C23-H23 & 120.0 \\
C24-C23-H23 & 120.0 & C23-C24-C19 & $120.57(17)$ \\
C23-C24-H24 & 119.7 & C19-C24-H24 & 119.7 \\
C18-C13-C14 & $119.24(17)$ & C18-C13-P1 & $123.16(14)$ \\
C14-C13-P1 & $117.60(13)$ & C15-C14-C13 & $120.50(19)$ \\
C15-C14-H14 & 119.8 & C13-C14-H14 & 119.8 \\
C16-C15-C14 & $119.9(2)$ & C16-C15-H15 & 120.1 \\
C14-C15-H15 & 120.1 & C15-C16-C17 & $120.08(19)$ \\
C15-C16-H16 & 120.0 & C17-C16-H16 & 120.0 \\
C16-C17-C18 & $120.2(2)$ & C16-C17-H17 & 119.9 \\
C18-C17-H17 & 119.9 & C13-C18-C17 & $120.11(19)$ \\
C25-H18 & 119.9 & C17-C18-H18 & 119.9 \\
C25 & $118.89(16)$ & C26-C25-P2 & $122.66(14)$ \\
\hline & $118.44(13)$ & C25-C26-C27 & $120.40(19)$ \\
\hline & 119.8 & C27-C26-H26 & 119.8 \\
\hline
\end{tabular}




\begin{tabular}{|c|c|c|c|}
\hline $\mathrm{C} 28-\mathrm{C} 27-\mathrm{C} 26$ & $120.2(2)$ & $\mathrm{C} 28-\mathrm{C} 27-\mathrm{H} 27$ & 119.9 \\
\hline $\mathrm{C} 26-\mathrm{C} 27-\mathrm{H} 27$ & 119.9 & $\mathrm{C} 29-\mathrm{C} 28-\mathrm{C} 27$ & $119.69(18)$ \\
\hline $\mathrm{C} 29-\mathrm{C} 28-\mathrm{H} 28$ & 120.2 & $\mathrm{C} 27-\mathrm{C} 28-\mathrm{H} 28$ & 120.2 \\
\hline C28-C29-C30 & $120.43(19)$ & $\mathrm{C} 28-\mathrm{C} 29-\mathrm{H} 29$ & 119.8 \\
\hline C30-C29-H29 & 119.8 & $\mathrm{C} 29-\mathrm{C} 30-\mathrm{C} 25$ & $120.36(18)$ \\
\hline C29-C30-H30 & 119.8 & $\mathrm{C} 25-\mathrm{C} 30-\mathrm{H} 30$ & 119.8 \\
\hline C32-C31-C36 & $119.15(16)$ & C32-C31-P2 & $118.85(13)$ \\
\hline C36-C31-P2 & $121.94(13)$ & C31-C32-C33 & $120.45(18)$ \\
\hline C31-C32-H32 & 119.8 & C33-C32-H32 & 119.8 \\
\hline C34-C33-C32 & $120.11(18)$ & C34-C33-H33 & 119.9 \\
\hline C32-C33-H33 & 119.9 & C33-C34-C35 & $119.85(18)$ \\
\hline C33-C34-H34 & 120.1 & C35-C34-H34 & 120.1 \\
\hline C36-C35-C34 & $120.48(18)$ & C36-C35-H35 & 119.8 \\
\hline C34-C35-H35 & 119.8 & C35-C36-C31 & $119.94(18)$ \\
\hline C35-C36-H36 & 120.0 & C31-C36-H36 & 120.0 \\
\hline C38-C37-C41 & $107.31(15)$ & C38-C37-P1 & $127.58(13)$ \\
\hline C41-C37-P1 & $125.11(13)$ & $\mathrm{C} 38-\mathrm{C} 37-\mathrm{Fe} 1$ & $69.79(9)$ \\
\hline $\mathrm{C} 41-\mathrm{C} 37-\mathrm{Fe} 1$ & $69.04(9)$ & $\mathrm{P} 1-\mathrm{C} 37-\mathrm{Fe} 1$ & $125.91(8)$ \\
\hline C39-C38-C37 & $107.84(16)$ & $\mathrm{C} 39-\mathrm{C} 38-\mathrm{Fe} 1$ & $69.92(10)$ \\
\hline C37-C38-Fe1 & $69.23(9)$ & C39-C38-H38 & 126.1 \\
\hline C37-C38-H38 & 126.1 & Fe1-C38-H38 & 126.1 \\
\hline C40-C39-C38 & $108.65(16)$ & $\mathrm{C} 40-\mathrm{C} 39-\mathrm{Fe} 1$ & $69.66(10)$ \\
\hline C38-C39-Fe1 & $69.51(10)$ & C40-C39-H39 & 125.7 \\
\hline C38-C39-H39 & 125.7 & Fe1-C39-H39 & 125.7 \\
\hline C39-C40-C41 & $108.20(16)$ & C39-C40-Fe1 & $70.12(10)$ \\
\hline C41-C40-Fe1 & $69.01(10)$ & C39-C40-H40 & 125.9 \\
\hline C41-C40-H40 & 125.9 & $\mathrm{Fe} 1-\mathrm{C} 40-\mathrm{H} 40$ & 125.9 \\
\hline $\mathrm{C} 40-\mathrm{C} 41-\mathrm{C} 37$ & $107.99(16)$ & $\mathrm{C} 40-\mathrm{C} 41-\mathrm{Fe} 1$ & $70.18(10)$ \\
\hline C37-C41-Fe1 & $69.69(9)$ & $\mathrm{C} 40-\mathrm{C} 41-\mathrm{H} 41$ & 126.0 \\
\hline C37-C41-H41 & 126.0 & Fe1-C41-H41 & 126.0 \\
\hline
\end{tabular}




$\begin{array}{llll}\text { C46-C42-C43 } & 107.67(15) & \text { C46-C42-P2 } & 129.66(13) \\ \text { C43-C42-P2 } & 122.39(13) & \text { C46-C42-Fe1 } & 70.27(10) \\ \text { C43-C42-Fe1 } & 69.57(9) & \text { P2-C42-Fe1 } & 120.86(8) \\ \text { C44-C43-C42 } & 107.49(15) & \text { C44-C43-Fe1 } & 70.61(10) \\ \text { C42-C43-Fe1 } & 68.96(9) & \text { C44-C43-H43 } & 126.3 \\ \text { C42-C43-H43 } & 126.3 & \text { Fe1-C43-H43 } & 126.3 \\ \text { C45-C44-C43 } & 108.54(16) & \text { C45-C44-Fe1 } & 70.08(10) \\ \text { C43-C44-Fe1 } & 68.66(9) & \text { C45-C44-H44 } & 125.7 \\ \text { C43-C44-H44 } & 125.7 & \text { Fe1-C44-H44 } & 125.7 \\ \text { C44-C45-C46 } & 108.38(16) & \text { C44-C45-Fe1 } & 69.69(10) \\ \text { C46-C45-Fe1 } & 69.24(10) & \text { C44-C45-H45 } & 125.8 \\ \text { C46-C45-H45 } & 125.8 & \text { Fe1-C45-H45 } & 125.8 \\ \text { C45-C46-C42 } & 107.91(16) & \text { C45-C46-Fe1 } & 70.40(10) \\ \text { C42-C46-Fe1 } & 68.49(9) & \text { C45-C46-H46 } & 126.0 \\ \text { C42-C46-H46 } & 126.0 & \text { Fe1-C46-H46 } & 126.0\end{array}$

Table S7f. Torsion angles $\left({ }^{\circ}\right)$ for $8 \mathrm{ca}$.

$\begin{array}{llll}\text { P1-Ni1-O1-C1 } & -8.32(14) & \text { P2-Ni1-O1-C1 } & -177.24(8) \\ \text { Ni1-O1-C1-C2 } & 175.9(2) & \text { P1-Ni1-C1-O1 } & 176.06(7) \\ \text { P2-Ni1-C1-O1 } & 4.29(13) & \text { O1-Ni1-C1-C2 } & -172.7(4) \\ \text { P1-Ni1-C1-C2 } & 3.4(4) & \text { P2-Ni1-C1-C2 } & -168.4(3) \\ \text { O1-C1-C2-C7 } & -2.0(3) & \text { Ni1-C1-C2-C7 } & 168.4(3) \\ \text { O1-C1-C2-C3 } & -179.77(18) & \text { Ni1-C1-C2-C3 } & -9.4(4) \\ \text { C1-C2-C3-C4 } & -112.36(19) & \text { C7-C2-C3-C4 } & 69.8(2) \\ \text { C2-C3-C4-C5 } & 63.1(2) & \text { C2-C3-C4-C6 } & -173.71(16) \\ \text { C1-C2-C7-C8 } & 1.6(3) & \text { C3-C2-C7-C8 } & 179.45(17) \\ \text { C1-C2-C7-C12 } & -178.16(16) & \text { C3-C2-C7-C12 } & -0.3(2) \\ \text { C12-C7-C8-C9 } & -0.3(3) & \text { C2-C7-C8-C9 } & 179.86(18) \\ \text { C7-C8-C9-C10 } & -0.2(3) & \text { C8-C9-C10-F1 } & -179.67(19) \\ \text { C8-C9-C10-C11 } & 0.2(3) & \text { C9-C10-C11-C12 } & 0.3(3) \\ & & & \text { S132 }\end{array}$




\begin{tabular}{|c|c|c|c|}
\hline F1-C10-C11-C12 & $-179.82(18)$ & $\mathrm{C} 10-\mathrm{C} 11-\mathrm{C} 12-\mathrm{C} 7$ & $-0.9(3)$ \\
\hline C8-C7-C12-C11 & $0.9(3)$ & $\mathrm{C} 2-\mathrm{C} 7-\mathrm{C} 12-\mathrm{C} 11$ & $-179.33(17)$ \\
\hline C37-P1-C19-C20 & $-146.80(14)$ & C13-P1-C19-C20 & $108.77(14)$ \\
\hline Ni1-P1-C19-C20 & $-19.69(15)$ & C37-P1-C19-C24 & $28.09(15)$ \\
\hline C13-P1-C19-C24 & $-76.34(15)$ & Ni1-P1-C19-C24 & $155.20(12)$ \\
\hline C24-C19-C20-C21 & $-0.9(3)$ & $\mathrm{P} 1-\mathrm{C} 19-\mathrm{C} 20-\mathrm{C} 21$ & $174.20(15)$ \\
\hline C19-C20-C21-C22 & $-0.1(3)$ & $\mathrm{C} 20-\mathrm{C} 21-\mathrm{C} 22-\mathrm{C} 23$ & $0.9(3)$ \\
\hline $\mathrm{C} 21-\mathrm{C} 22-\mathrm{C} 23-\mathrm{C} 24$ & $-0.7(3)$ & $\mathrm{C} 22-\mathrm{C} 23-\mathrm{C} 24-\mathrm{C} 19$ & $-0.3(3)$ \\
\hline C20-C19-C24-C23 & $1.1(3)$ & $\mathrm{P} 1-\mathrm{C} 19-\mathrm{C} 24-\mathrm{C} 23$ & $-173.83(14)$ \\
\hline C37-P1-C13-C18 & $-98.09(16)$ & C19-P1-C13-C18 & $6.78(18)$ \\
\hline Ni1-P1-C13-C18 & $133.46(14)$ & C37-P1-C13-C14 & $82.70(14)$ \\
\hline C19-P1-C13-C14 & $-172.43(14)$ & Ni1-P1-C13-C14 & $-45.75(15)$ \\
\hline C18-C13-C14-C15 & $0.1(3)$ & P1-C13-C14-C15 & $179.35(15)$ \\
\hline C13-C14-C15-C16 & $-1.2(3)$ & C14-C15-C16-C17 & $0.8(3)$ \\
\hline C15-C16-C17-C18 & $0.6(4)$ & C14-C13-C18-C17 & $1.3(3)$ \\
\hline P1-C13-C18-C17 & $-177.89(17)$ & C16-C17-C18-C13 & $-1.7(4)$ \\
\hline C42-P2-C25-C26 & $-11.80(18)$ & $\mathrm{C} 31-\mathrm{P} 2-\mathrm{C} 25-\mathrm{C} 26$ & $-120.93(17)$ \\
\hline Ni1-P2-C25-C26 & $109.12(16)$ & C42-P2-C25-C30 & $167.03(14)$ \\
\hline C31-P2-C25-C30 & $57.89(15)$ & Ni1-P2-C25-C30 & $-72.05(14)$ \\
\hline C30-C25-C26-C27 & $-0.6(3)$ & $\mathrm{P} 2-\mathrm{C} 25-\mathrm{C} 26-\mathrm{C} 27$ & $178.22(17)$ \\
\hline $\mathrm{C} 25-\mathrm{C} 26-\mathrm{C} 27-\mathrm{C} 28$ & $-0.3(4)$ & C26-C27-C28-C29 & $0.7(4)$ \\
\hline C27-C28-C29-C30 & $-0.2(3)$ & $\mathrm{C} 28-\mathrm{C} 29-\mathrm{C} 30-\mathrm{C} 25$ & $-0.7(3)$ \\
\hline $\mathrm{C} 26-\mathrm{C} 25-\mathrm{C} 30-\mathrm{C} 29$ & $1.1(3)$ & $\mathrm{P} 2-\mathrm{C} 25-\mathrm{C} 30-\mathrm{C} 29$ & $-177.81(14)$ \\
\hline C42-P2-C31-C32 & $118.84(14)$ & C25-P2-C31-C32 & $-134.00(14)$ \\
\hline Ni1-P2-C31-C32 & $-7.51(16)$ & C42-P2-C31-C36 & $-63.86(16)$ \\
\hline C25-P2-C31-C36 & $43.30(16)$ & Ni1-P2-C31-C36 & $169.79(12)$ \\
\hline C36-C31-C32-C33 & $1.0(3)$ & P2-C31-C32-C33 & $178.35(15)$ \\
\hline C31-C32-C33-C34 & $-0.6(3)$ & C32-C33-C34-C35 & $0.0(3)$ \\
\hline C33-C34-C35-C36 & $0.3(3)$ & C34-C35-C36-C31 & $0.0(3)$ \\
\hline C32-C31-C36-C35 & $-0.7(3)$ & P2-C31-C36-C35 & -177 \\
\hline
\end{tabular}




\begin{tabular}{|c|c|c|c|}
\hline 19-P1-C37-C38 & $-133.00(15)$ & C13-P1-C37-C38 & $-24.46(16)$ \\
\hline i1-P1-C37-C38 & $2.95(14)$ & C19-P1-C37-C41 & $47.67(15)$ \\
\hline 13-P1-C37-C41 & $156.21(14)$ & Ni1-P1-C37-C41 & $-76.38(15)$ \\
\hline 19-P1-C37-Fe1 & $35.91(10)$ & C13-P & $115.55(11)$ \\
\hline Ji1-P1-C37-Fe1 & $11.86(13)$ & $\mathrm{C} 41-\mathrm{C} 37-\mathrm{C} 38-\mathrm{C} 39$ & $-0.40(18)$ \\
\hline $1-\mathrm{C} 37-\mathrm{C} 38-\mathrm{C} 39$ & $-179.83(12)$ & Fe1-C37-C38-C39 & $-59.48(12)$ \\
\hline 441-C37-C38-Fe1 & $59.07(11)$ & $\mathrm{P} 1-\mathrm{C} 3$ & -120.36 \\
\hline C37-C38-C39-C40 & 0.22( & 9-C40 & $-58.82(12)$ \\
\hline C37-C38-C39-Fe1 & $59.04(11)$ & C38-C39-C40-C41 & $0.0(2)$ \\
\hline e1-C39-C40-C41 & $-58.68(12)$ & C38-C39-C40-Fe1 & $58.73(12)$ \\
\hline C39-C40-C41-C37 & $-0.30(19)$ & $\mathrm{Fe} 1-\mathrm{C} 40-\mathrm{C} 41-\mathrm{C} 37$ & $-59.67(11)$ \\
\hline $39-\mathrm{C} 40-\mathrm{C}$ & $59.3^{\circ}$ & $-\mathrm{C} 40$ & 0.43( \\
\hline 1-C37-C41-C40 & $179.88(12)$ & Fe1-C37-C41-C40 & $59.98(12)$ \\
\hline $\mathrm{C} 38-\mathrm{C} 37$ & -59.5 & $\mathrm{P} 1-\mathrm{C}$ & 119. \\
\hline $\mathrm{C} 31-\mathrm{P} 2-\mathrm{C} 42-\mathrm{C} 46$ & $12.55(17)$ & $\mathrm{C} 25-\mathrm{P} 2-\mathrm{C} 42-\mathrm{C} 46$ & $-92.21(16)$ \\
\hline Vi1-P2-C42-C46 & 144.2 & C31-1 & -160.68 \\
\hline $\mathrm{C} 25-\mathrm{P} 2-\mathrm{C} 42-\mathrm{C} 43$ & $94.56(14)$ & Ni1-P2-C42-C43 & $-28.96(15)$ \\
\hline $\mathrm{C} 31-\mathrm{P} 2-\mathrm{C} 42-\mathrm{Fe} 1$ & $-76.56(11)$ & $\mathrm{C} 25-\mathrm{P} 2-\mathrm{C}$ & 178.6 \\
\hline Ni1-P2-C42-Fe1 & $55.16(10)$ & C46-C42-C43-C44 & $0.17(18)$ \\
\hline 2-C42-C43-C44 & $174.71(12)$ & Fe1-C42-C43-C44 & $60.38(11)$ \\
\hline $46-\mathrm{C} 42-\mathrm{C} 43-\mathrm{Fe} 1$ & $-60.21(11)$ & $\mathrm{P} 2-\mathrm{C} 42-\mathrm{C} 43-\mathrm{Fe} 1$ & $114.32(12)$ \\
\hline C42-C43-C44-C45 & $-0.46(19)$ & $\mathrm{Fe} 1-\mathrm{C} 43-\mathrm{C} 44-\mathrm{C} 45$ & $58.87(12)$ \\
\hline $\mathrm{C} 42-\mathrm{C} 43-\mathrm{C} 44-\mathrm{Fe} 1$ & $-59.34(11)$ & C43-C44-C45-C46 & $0.6(2)$ \\
\hline e1-C44-C45-C46 & 58.59 & $\mathrm{C} 43-\mathrm{C} 44-\mathrm{C} 45-\mathrm{Fe} 1$ & $-58.01(12)$ \\
\hline 5-C46-C42 & $-0.5(2$ & 46-C42 & $58.40(11)$ \\
\hline C44-C45-C46-Fe1 & $-58.87(12)$ & $\mathrm{C} 43-\mathrm{C} 42-\mathrm{C} 46-\mathrm{C} 45$ & $0.18(1$ \\
\hline 2-C42-C46-C45 & $-173.82(13)$ & $\mathrm{Fe} 1-\mathrm{C} 42-\mathrm{C} 46-\mathrm{C} 45$ & $-59.59(12)$ \\
\hline $43-\mathrm{C} 42-\mathrm{C} 46-\mathrm{Fe} 1$ & $59.77(11)$ & $\mathrm{P} 2-\mathrm{C} 42-\mathrm{C} 46-\mathrm{Fe}$ & -114.23 \\
\hline
\end{tabular}

Table S7g. Anisotropic atomic displacement parameters $\left(\AA^{2}\right)$ for $8 \mathrm{ca}$. 
The anisotropic atomic displacement factor exponent takes the form: $-2 \pi^{2}$ [ $\left.\mathrm{h}^{2} \mathrm{a}^{* 2} \mathrm{U}_{11}+\ldots+2 \mathrm{~h} \mathrm{k} \mathrm{a}^{*} \mathrm{~b}^{*} \mathrm{U}_{12}\right]$

\begin{tabular}{|c|c|c|c|c|c|c|}
\hline & $\mathbf{U}_{11}$ & $\mathbf{U}_{22}$ & $\mathbf{U}_{33}$ & $\mathbf{U}_{23}$ & $\mathbf{U}_{13}$ & $\mathbf{U}_{12}$ \\
\hline Ni1 & $0.01535(10)$ & $0.01730(10)$ & $0.01633(11)$ & $-0.00136(8)$ & $0.00575(8)$ & $0.00006(7)$ \\
\hline $\mathrm{Fe} 1$ & $0.02003(12)$ & $0.02164(12)$ & $0.01654(12)$ & $-0.00246(9)$ & $0.00604(9)$ & $-0.00303(9)$ \\
\hline P1 & $0.01467(18)$ & $0.0185(2)$ & $0.0169(2)$ & $-0.00177(15)$ & $0.00457(15)$ & $-0.00060(15)$ \\
\hline $\mathrm{P} 2$ & $0.01749(19)$ & $0.0173(2)$ & $0.0191(2)$ & $-0.00179(15)$ & $0.00635(16)$ & $-0.00120(15)$ \\
\hline F1 & $0.0322(7)$ & $0.0788(10)$ & $0.0647(9)$ & $-0.0348(8)$ & $0.0261(6)$ & $0.0002(6)$ \\
\hline $\mathrm{O} 1$ & $0.0203(6)$ & $0.0204(6)$ & $0.0236(6)$ & $-0.0029(5)$ & $0.0112(5)$ & $-0.0011(4)$ \\
\hline $\mathrm{C} 1$ & $0.0196(7)$ & $0.0222(8)$ & $0.0185(8)$ & $0.0020(6)$ & $0.0073(6)$ & $0.0008(6)$ \\
\hline $\mathrm{C} 2$ & $0.0240(8)$ & $0.0204(8)$ & $0.0201(8)$ & $-0.0004(6)$ & $0.0081(7)$ & $0.0008(6)$ \\
\hline $\mathrm{C} 3$ & $0.0279(9)$ & $0.0274(9)$ & $0.0254(9)$ & $-0.0062(7)$ & $0.0121(7)$ & $-0.0048(7)$ \\
\hline $\mathrm{C} 4$ & $0.0228(9)$ & $0.0370(10)$ & $0.0347(10)$ & $-0.0109(8)$ & $0.0085(8)$ & $-0.0026(8)$ \\
\hline $\mathrm{C} 5$ & $0.0441(13)$ & $0.0470(14)$ & $0.0582(16)$ & $-0.0069(12)$ & $-0.0064(12)$ & $0.0078(11)$ \\
\hline C6 & $0.0287(10)$ & $0.0508(13)$ & $0.0593(15)$ & $-0.0270(12)$ & $0.0122(10)$ & $-0.0120(9)$ \\
\hline C7 & $0.0237(8)$ & $0.0210(8)$ & $0.0201(8)$ & $0.0008(6)$ & $0.0071(7)$ & $0.0032(6)$ \\
\hline C8 & $0.0251(9)$ & $0.0326(10)$ & $0.0326(10)$ & $-0.0094(8)$ & $0.0110(8)$ & $-0.0016(7)$ \\
\hline C9 & $0.0231(9)$ & $0.0416(11)$ & $0.0408(11)$ & $-0.0107(9)$ & $0.0124(8)$ & $-0.0032(8)$ \\
\hline $\mathrm{C} 10$ & $0.0231(9)$ & $0.0455(12)$ & $0.0377(11)$ & $-0.0070(9)$ & $0.0130(8)$ & $0.0082(8)$ \\
\hline $\mathrm{C} 11$ & $0.0333(10)$ & $0.0301(10)$ & $0.0317(10)$ & $-0.0084(8)$ & $0.0083(8)$ & $0.0074(8)$ \\
\hline $\mathrm{C} 12$ & $0.0288(9)$ & $0.0227(9)$ & $0.0285(9)$ & $-0.0012(7)$ & $0.0088(8)$ & $0.0020(7)$ \\
\hline C19 & $0.0154(7)$ & $0.0199(8)$ & $0.0195(8)$ & $-0.0033(6)$ & $0.0029(6)$ & $0.0007(6)$ \\
\hline $\mathrm{C} 20$ & $0.0193(8)$ & $0.0299(9)$ & $0.0231(9)$ & $-0.0003(7)$ & $0.0057(7)$ & $0.0004(7)$ \\
\hline $\mathrm{C} 21$ & $0.0260(9)$ & $0.0399(11)$ & $0.0286(10)$ & $0.0076(8)$ & $0.0030(8)$ & $0.0024(8)$ \\
\hline $\mathrm{C} 22$ & $0.0234(9)$ & $0.0342(10)$ & $0.0391(11)$ & $-0.0003(9)$ & $-0.0006(8)$ & $0.0075(8)$ \\
\hline $\mathrm{C} 23$ & $0.0180(8)$ & $0.0326(10)$ & $0.0382(11)$ & $-0.0093(8)$ & $0.0070(8)$ & $0.0018(7)$ \\
\hline $\mathrm{C} 24$ & $0.0198(8)$ & $0.0267(9)$ & $0.0256(9)$ & $-0.0065(7)$ & $0.0074(7)$ & $-0.0021(7)$ \\
\hline $\mathrm{C} 13$ & $0.0240(8)$ & $0.0196(8)$ & $0.0213(8)$ & $-0.0010(6)$ & $0.0106(7)$ & $-0.0013(6)$ \\
\hline C14 & $0.0359(10)$ & $0.0272(9)$ & $0.0247(9)$ & $0.0004(7)$ & $0.0051(8)$ & $0.0056(8)$ \\
\hline $\mathrm{C} 15$ & $0.0616(14)$ & $0.0267(10)$ & $0.0324(11)$ & $0.0068(8)$ & $0.0166(10)$ & $0.0127(10)$ \\
\hline
\end{tabular}




$\begin{array}{lllllll} & \mathbf{U}_{11} & \mathbf{U}_{22} & \mathbf{U}_{33} & \mathbf{U}_{23} & \mathbf{U}_{13} & \mathbf{U}_{12} \\ \text { C16 } & 0.0674(15) & 0.0211(9) & 0.0642(15) & 0.0000(10) & 0.0416(13) & -0.0059(10) \\ \text { C17 } & 0.0402(12) & 0.0326(11) & 0.0762(17) & -0.0140(11) & 0.0282(12) & -0.0152(9) \\ \text { C18 } & 0.0249(9) & 0.0272(10) & 0.0522(13) & -0.0082(9) & 0.0143(9) & -0.0038(7) \\ \text { C25 } & 0.0209(8) & 0.0181(8) & 0.0261(9) & -0.0042(7) & 0.0095(7) & -0.0020(6) \\ \text { C26 } & 0.0243(9) & 0.0464(12) & 0.0355(11) & 0.0097(9) & 0.0056(8) & -0.0055(8) \\ \text { C27 } & 0.0205(9) & 0.0592(14) & 0.0479(13) & 0.0058(11) & 0.0035(9) & -0.0086(9) \\ \text { C28 } & 0.0282(10) & 0.0360(11) & 0.0517(13) & -0.0071(9) & 0.0194(9) & -0.0118(8) \\ \text { C29 } & 0.0398(11) & 0.0303(10) & 0.0374(11) & -0.0013(8) & 0.0231(9) & -0.0066(8) \\ \text { C30 } & 0.0291(9) & 0.0297(9) & 0.0257(9) & -0.0023(7) & 0.0116(8) & -0.0035(7) \\ \text { C31 } & 0.0245(8) & 0.0194(8) & 0.0229(8) & 0.0003(6) & 0.0101(7) & 0.0004(6) \\ \text { C32 } & 0.0258(9) & 0.0229(8) & 0.0288(9) & 0.0019(7) & 0.0069(7) & 0.0001(7) \\ \text { C33 } & 0.0285(9) & 0.0349(10) & 0.0392(11) & 0.0085(9) & 0.0066(8) & 0.0075(8) \\ \text { C34 } & 0.0494(12) & 0.0258(10) & 0.0400(11) & 0.0066(8) & 0.0200(10) & 0.0140(9) \\ \text { C35 } & 0.0494(12) & 0.0213(9) & 0.0320(10) & -0.0026(8) & 0.0163(9) & 0.0029(8) \\ \text { C36 } & 0.0317(9) & 0.0239(9) & 0.0267(9) & -0.0024(7) & 0.0095(8) & -0.0004(7) \\ \text { C37 } & 0.0156(7) & 0.0233(8) & 0.0189(8) & -0.0025(6) & 0.0053(6) & -0.0027(6) \\ \text { C38 } & 0.0215(8) & 0.0261(9) & 0.0225(9) & -0.0003(7) & 0.0065(7) & -0.0055(7) \\ \text { C39 } & 0.0264(9) & 0.0375(10) & 0.0209(9) & -0.0010(7) & 0.0119(7) & -0.0083(8) \\ \text { C40 } & 0.0261(9) & 0.0336(10) & 0.0260(9) & -0.0056(8) & 0.0133(7) & -0.0012(7) \\ \text { C41 } & 0.0202(8) & 0.0267(9) & 0.0228(9) & -0.0025(7) & 0.0073(7) & 0.0005(7) \\ \text { C42 } & 0.0190(7) & 0.0204(8) & 0.0196(8) & -0.0024(6) & 0.0054(6) & -0.0052(6) \\ \text { C43 } & 0.0171(7) & 0.0210(8) & 0.0254(9) & -0.0004(7) & 0.0049(7) & -0.0015(6) \\ \text { C44 } & 0.0224(8) & 0.0294(9) & 0.0210(8) & 0.0024(7) & 0.0008(7) & -0.0041(7) \\ \text { C45 } & 0.0296(9) & 0.0319(10) & 0.0181(8) & -0.0052(7) & 0.0040(7) & -0.0087(7) \\ & 0.0259(8) & 0.0240(8) & 0.0229(9) & -0.0059(7) & 0.0078(7) & -0.0058(7)\end{array}$

Table S7h. Hydrogen atomic coordinates and isotropic atomic displacement parameters $\left(\AA^{2}\right)$ for 8 ca. 


\begin{tabular}{|c|c|c|c|c|}
\hline & $\mathbf{x} / \mathbf{a}$ & $\mathbf{y} / \mathbf{b}$ & $\mathbf{z} / \mathbf{c}$ & $\mathrm{U}(\mathrm{eq})$ \\
\hline $\mathrm{H} 3 \mathrm{~A}$ & 0.6313 & 0.5190 & 0.6903 & 0.031 \\
\hline H3B & 0.7153 & 0.4619 & 0.6672 & 0.031 \\
\hline H4 & 0.7034 & 0.4823 & 0.8223 & 0.038 \\
\hline $\mathrm{H} 5 \mathrm{~A}$ & 0.8785 & 0.3972 & 0.7760 & 0.082 \\
\hline H5B & 0.7536 & 0.3704 & 0.7967 & 0.082 \\
\hline $\mathrm{H} 5 \mathrm{C}$ & 0.8684 & 0.4049 & 0.8623 & 0.082 \\
\hline H6A & 0.9116 & 0.5286 & 0.8490 & 0.07 \\
\hline H6B & 0.8123 & 0.5708 & 0.7824 & 0.07 \\
\hline $\mathrm{H} 6 \mathrm{C}$ & 0.9082 & 0.5192 & 0.7604 & 0.07 \\
\hline H8 & 0.3276 & 0.3623 & 0.6725 & 0.035 \\
\hline H9 & 0.1622 & 0.3854 & 0.7263 & 0.041 \\
\hline H1 1 & 0.3550 & 0.5471 & 0.8328 & 0.038 \\
\hline H12 & 0.5193 & 0.5253 & 0.7777 & 0.032 \\
\hline $\mathrm{H} 20$ & 0.8352 & 0.2916 & 0.6862 & 0.029 \\
\hline $\mathrm{H} 21$ & 1.0147 & 0.2334 & 0.7619 & 0.039 \\
\hline $\mathrm{H} 22$ & 1.1970 & 0.2222 & 0.7192 & 0.041 \\
\hline $\mathrm{H} 23$ & 1.2021 & 0.2711 & 0.6033 & 0.036 \\
\hline $\mathrm{H} 24$ & 1.0235 & 0.3297 & 0.5279 & 0.029 \\
\hline H14 & 0.6051 & 0.4662 & 0.4845 & 0.036 \\
\hline H15 & 0.6311 & 0.5817 & 0.4887 & 0.048 \\
\hline H16 & 0.8306 & 0.6281 & 0.5536 & 0.055 \\
\hline H17 & 1.0051 & 0.5593 & 0.6118 & 0.057 \\
\hline H18 & 0.9819 & 0.4436 & 0.6033 & 0.041 \\
\hline H26 & 0.2542 & 0.2330 & 0.3834 & 0.043 \\
\hline $\mathrm{H} 27$ & 0.0613 & 0.1853 & 0.3903 & 0.053 \\
\hline $\mathrm{H} 28$ & 0.0572 & 0.1229 & 0.4983 & 0.044 \\
\hline H29 & 0.2448 & 0.1099 & 0.6005 & 0.04 \\
\hline H30 & 0.4370 & 0.1584 & 0.5952 & 0.033 \\
\hline H32 & 0.7718 & 0.1960 & 0.5770 & 0.031 \\
\hline
\end{tabular}




$\begin{array}{lllll} & \mathbf{x} / \mathbf{a} & \mathbf{y} / \mathbf{b} & \mathbf{z} / \mathbf{c} & \mathbf{U}(\mathbf{e q}) \\ \text { H33 } & 0.8957 & 0.0989 & 0.5958 & 0.042 \\ \text { H34 } & 0.8126 & 0.0021 & 0.5281 & 0.044 \\ \text { H35 } & 0.6054 & 0.0023 & 0.4412 & 0.04 \\ \text { H36 } & 0.4811 & 0.0991 & 0.4209 & 0.033 \\ \text { H38 } & 0.7298 & 0.4297 & 0.3779 & 0.028 \\ \text { H39 } & 0.7658 & 0.3649 & 0.2616 & 0.033 \\ \text { H40 } & 0.8252 & 0.2433 & 0.3040 & 0.033 \\ \text { H41 } & 0.8263 & 0.2310 & 0.4475 & 0.028 \\ \text { H43 } & 0.4336 & 0.3627 & 0.3916 & 0.026 \\ \text { H44 } & 0.4282 & 0.3699 & 0.2471 & 0.031 \\ \text { H45 } & 0.4910 & 0.2548 & 0.2050 & 0.033 \\ \text { H46 } & 0.5347 & 0.1747 & 0.3223 & 0.029\end{array}$


Table S8a. Sample and crystal data for $8 \mathrm{cb}$.

\begin{tabular}{|c|c|c|}
\hline Identification code & \multicolumn{2}{|l|}{$8 \mathrm{cb}$} \\
\hline Chemical formula & \multicolumn{2}{|l|}{$\mathrm{C}_{46} \mathrm{H}_{42} \mathrm{FeNiOP}_{2}$} \\
\hline Formula weight & \multicolumn{2}{|l|}{$787.29 \mathrm{~g} / \mathrm{mol}$} \\
\hline Temperature & \multicolumn{2}{|l|}{$150(1) \mathrm{K}$} \\
\hline Wavelength & \multicolumn{2}{|l|}{$0.71073 \AA$} \\
\hline Crystal size & \multicolumn{2}{|c|}{$0.126 \times 0.210 \times 0.252 \mathrm{~mm}$} \\
\hline Crystal habit & \multicolumn{2}{|l|}{ orange prism } \\
\hline Crystal system & \multicolumn{2}{|l|}{ monoclinic } \\
\hline Space group & \multicolumn{2}{|l|}{ P $121 / \mathrm{c} 1$} \\
\hline \multirow[t]{3}{*}{ Unit cell dimensions } & $a=10.8021(7) \AA$ & $\alpha=90^{\circ}$ \\
\hline & $\mathrm{b}=19.9889(13) \AA$ & $\beta=107.071(4)^{\circ}$ \\
\hline & $\mathrm{c}=18.0037(13) \AA$ & $\gamma=90^{\circ}$ \\
\hline Volume & \multicolumn{2}{|l|}{$3716.1(4) \AA^{3}$} \\
\hline $\mathbf{Z}$ & \multicolumn{2}{|l|}{4} \\
\hline Density (calculated) & \multicolumn{2}{|l|}{$1.407 \mathrm{~g} / \mathrm{cm}^{3}$} \\
\hline Absorption coefficient & \multicolumn{2}{|l|}{$1.019 \mathrm{~mm}^{-1}$} \\
\hline $\mathbf{F}(000)$ & \multicolumn{2}{|l|}{1640} \\
\hline
\end{tabular}

Table S8b. Data collection and structure refinement for $8 \mathrm{cb}$.

Theta range for data collection $\quad 1.97$ to $27.87^{\circ}$

Index ranges $\quad-14<=\mathrm{h}<=14,-26<=\mathrm{k}<=26,-23<=1<=23$

Reflections collected $\quad 96641$

Independent reflections $\quad 8870[\mathrm{R}(\mathrm{int})=0.0444]$

Coverage of independent reflections $100.0 \%$

Absorption correction multi-scan

Max. and min. transmission $\quad 0.8820$ and 0.7830

Structure solution technique direct methods

Structure solution program $\quad$ SHELXT-2014/9 (Sheldrick, 2014) 


\begin{tabular}{|c|c|}
\hline Refinement method & Full-matrix least-squares on $\mathrm{F}^{2}$ \\
\hline Refinement program & SHELXL-2014/6 (Sheldrick, 2014) \\
\hline Function minimized & $\Sigma \mathrm{w}\left(\mathrm{F}_{\mathrm{o}}^{2}-\mathrm{F}_{\mathrm{c}}^{2}\right)^{2}$ \\
\hline Data / restraints / parameters & $8870 / 0 / 462$ \\
\hline Goodness-of-fit on $F^{2}$ & 1.065 \\
\hline$\Delta / \sigma_{\max }$ & 0.002 \\
\hline \multirow[t]{2}{*}{ Final $R$ indices } & 7279 data; $\mathrm{I}>2 \sigma(\mathrm{I}) \mathrm{R} 1=0.0308, \mathrm{wR} 2=0.0770$ \\
\hline & $\mathrm{R} 1=0.0419, \mathrm{wR} 2=0.0811$ \\
\hline \multirow{2}{*}{ Weighting scheme } & $\mathrm{w}=1 /\left[\sigma^{2}\left(\mathrm{~F}_{\mathrm{o}}^{2}\right)+(0.0394 \mathrm{P})^{2}+1.7678 \mathrm{P}\right]$ \\
\hline & where $\mathrm{P}=\left(\mathrm{F}_{\mathrm{o}}^{2}+2 \mathrm{~F}_{\mathrm{c}}^{2}\right) / 3$ \\
\hline Largest diff. peak and hole & 0.350 and $-0.338 \mathrm{e}^{-3}$ \\
\hline R.M.S. deviation from mean & $0.061 \mathrm{e}^{-3}$ \\
\hline
\end{tabular}

Table S8c. Atomic coordinates and equivalent isotropic atomic displacement parameters $\left(\AA^{2}\right)$ for

$8 c b$.

$\mathrm{U}(\mathrm{eq})$ is defined as one third of the trace of the orthogonalized $\mathrm{U}_{\mathrm{ij}}$ tensor.

$\begin{array}{lllll} & \mathbf{x} / \mathbf{a} & \mathbf{y} / \mathbf{b} & \mathbf{z} / \mathbf{c} & \mathbf{U}(\mathbf{e q}) \\ \mathrm{Ni1} & 0.40825(2) & 0.67910(2) & 0.43019(2) & 0.01606(6) \\ \mathrm{Fe} 1 & 0.37207(2) & 0.69801(2) & 0.64695(2) & 0.01944(7) \\ \mathrm{P} 2 & 0.48018(4) & 0.76583(2) & 0.50901(3) & 0.01730(9) \\ \mathrm{P} 1 & 0.23798(4) & 0.64526(2) & 0.45854(2) & 0.01679(9) \\ \mathrm{O} 1 & 0.53909(11) & 0.67071(6) & 0.38324(7) & 0.0203(3) \\ \mathrm{C} 1 & 0.45443(16) & 0.62411(9) & 0.35743(10) & 0.0192(3) \\ \mathrm{C} 2 & 0.44410(16) & 0.57132(9) & 0.30886(10) & 0.0207(3) \\ \mathrm{C} 3 & 0.32456(17) & 0.52765(9) & 0.29121(11) & 0.0257(4) \\ \mathrm{C} 4 & 0.23344(18) & 0.52980(10) & 0.20754(12) & 0.0323(4) \\ \mathrm{C} 5 & 0.1845(2) & 0.59932(13) & 0.18375(16) & 0.0532(7) \\ \mathrm{C} 6 & 0.1215(2) & 0.48128(14) & 0.19940(16) & 0.0538(7)\end{array}$




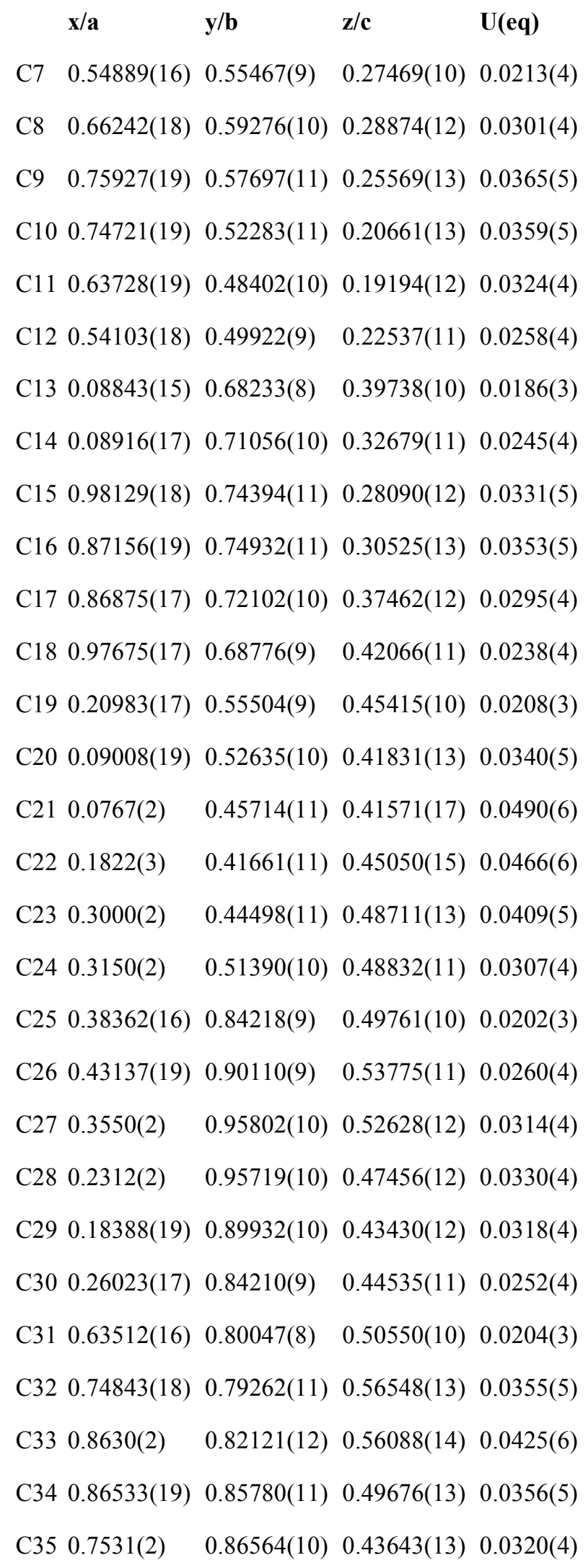




\begin{abstract}
$\begin{array}{llll}\mathbf{x} / \mathbf{a} & \mathbf{y} / \mathbf{b} & \mathbf{z} / \mathbf{c} & \mathbf{U}(\mathbf{e q})\end{array}$
$\begin{array}{llllll}\mathrm{C} 36 & 0.63847(18) & 0.83711(9) & 0.44026(11) & 0.0259(4)\end{array}$

$\begin{array}{llllll}\mathrm{C} 37 & 0.22576(15) & 0.66318(9) & 0.55539(10) & 0.0198(3)\end{array}$

$\begin{array}{llllll}\mathrm{C} 38 & 0.19290(16) & 0.72701(9) & 0.58097(11) & 0.0231(4)\end{array}$

$\begin{array}{llllll}\mathrm{C} 39 & 0.19456(17) & 0.72055(10) & 0.66018(11) & 0.0275(4)\end{array}$

$\begin{array}{llllll}\text { C40 0.22840(18) } & 0.65385(10) & 0.68383(11) & 0.0273(4)\end{array}$

$\begin{array}{llllll}\mathrm{C} 41 & 0.24843(16) & 0.61802(9) & 0.61995(10) & 0.0230(4)\end{array}$

$\begin{array}{llllll}\text { C42 } & 0.51168(16) & 0.74156(9) & 0.60895(10) & 0.0196(3)\end{array}$

$\begin{array}{llllll}\mathrm{C} 43 & 0.49455(17) & 0.77855(9) & 0.67346(10) & 0.0242(4)\end{array}$

$\begin{array}{llllll}\text { C44 0.51970(18) } & 0.73479(10) & 0.73820(11) & 0.0273(4)\end{array}$

$\begin{array}{llllll}\text { C45 0.55361(17) } & 0.67138(10) & 0.71503(11) & 0.0265(4)\end{array}$

$\begin{array}{llllll}\text { C46 0.54838(16) } & 0.67468(9) & 0.63572(10) & 0.0211(4)\end{array}$
\end{abstract}

\title{
Table S8d. Bond lengths (Å) for $8 \mathrm{cb}$.
}

$\begin{array}{llll}\text { Ni1-O1 } & 1.8551(12) & \text { Ni1-C1 } & 1.8860(17) \\ \text { Ni1-P1 } & 2.1569(5) & \text { Ni1-P2 } & 2.2305(5) \\ \text { Fe1-C42 } & 2.0262(17) & \text { Fe1-C46 } & 2.0277(17) \\ \text { Fe1-C38 } & 2.0355(18) & \text { Fe1-C37 } & 2.0422(17) \\ \text { Fe1-C41 } & 2.0483(18) & \text { Fe1-C43 } & 2.0492(18) \\ \text { Fe1-C39 } & 2.0505(18) & \text { Fe1-C45 } & 2.0543(18) \\ \text { Fe1-C40 } & 2.0576(18) & \text { Fe1-C44 } & 2.0601(18) \\ \text { P2-C42 } & 1.7971(18) & \text { P2-C25 } & 1.8257(18) \\ \text { P2-C31 } & 1.8294(17) & \text { P1-C37 } & 1.8223(18) \\ \text { P1-C13 } & 1.8240(17) & \text { P1-C19 } & 1.8267(18) \\ \text { O1-C1 } & 1.292(2) & \text { C1-C2 } & 1.354(2) \\ \text { C2-C7 } & 1.476(2) & \text { C2-C3 } & 1.513(2) \\ \text { C3-C4 } & 1.539(3) & \text { C3-H3A } & 0.99 \\ \text { C3-H3B } & 0.99 & \text { C4-C5 } & 1.504(3) \\ \text { C4-C6 } & 1.523(3) & \text { C4-H4 } & 1.0\end{array}$




\begin{tabular}{|c|c|c|c|}
\hline C5-H5A & 0.98 & C5-H5B & 0.98 \\
\hline C5-H5C & 0.98 & C6-H6A & 0.98 \\
\hline C6-H6B & 0.98 & C6-H6C & 0.98 \\
\hline $\mathrm{C} 7-\mathrm{C} 8$ & $1.402(3)$ & $\mathrm{C} 7-\mathrm{C} 12$ & $1.407(2)$ \\
\hline C8-C9 & $1.383(3)$ & C8-H8 & 0.95 \\
\hline C9-C10 & $1.379(3)$ & C9-H9 & 0.95 \\
\hline $\mathrm{C} 10-\mathrm{C} 11$ & $1.377(3)$ & C10-H10 & 0.95 \\
\hline C11-C12 & $1.379(3)$ & C11-H11 & 0.95 \\
\hline C12-H12 & 0.95 & C13-C14 & $1.393(3)$ \\
\hline C13-C18 & $1.393(2)$ & $\mathrm{C} 14-\mathrm{C} 15$ & $1.386(3)$ \\
\hline C14-H14 & 0.95 & $\mathrm{C} 15-\mathrm{C} 16$ & $1.384(3)$ \\
\hline C15-H15 & 0.95 & C16-C17 & $1.379(3)$ \\
\hline C16-H16 & 0.95 & $\mathrm{C} 17-\mathrm{C} 18$ & $1.386(3)$ \\
\hline C17-H17 & 0.95 & C18-H18 & 0.95 \\
\hline C19-C20 & $1.389(3)$ & C19-C24 & $1.391(3)$ \\
\hline $\mathrm{C} 20-\mathrm{C} 21$ & $1.390(3)$ & $\mathrm{C} 20-\mathrm{H} 20$ & 0.95 \\
\hline $\mathrm{C} 21-\mathrm{C} 22$ & $1.389(4)$ & $\mathrm{C} 21-\mathrm{H} 21$ & 0.95 \\
\hline C22-C23 & $1.372(3)$ & $\mathrm{C} 22-\mathrm{H} 22$ & 0.95 \\
\hline C23-C24 & $1.387(3)$ & $\mathrm{C} 23-\mathrm{H} 23$ & 0.95 \\
\hline C24-H24 & 0.95 & $\mathrm{C} 25-\mathrm{C} 30$ & $1.388(2)$ \\
\hline $\mathrm{C} 25-\mathrm{C} 26$ & $1.399(3)$ & $\mathrm{C} 26-\mathrm{C} 27$ & $1.385(3)$ \\
\hline C26-H26 & 0.95 & $\mathrm{C} 27-\mathrm{C} 28$ & $1.387(3)$ \\
\hline $\mathrm{C} 27-\mathrm{H} 27$ & 0.95 & $\mathrm{C} 28-\mathrm{C} 29$ & $1.381(3)$ \\
\hline C28-H28 & 0.95 & C29-C30 & $1.390(3)$ \\
\hline C29-H29 & 0.95 & C30-H30 & 0.95 \\
\hline C31-C32 & $1.383(3)$ & C31-C36 & $1.394(3)$ \\
\hline C32-C33 & $1.387(3)$ & $\mathrm{C} 32-\mathrm{H} 32$ & 0.95 \\
\hline C33-C34 & $1.373(3)$ & C33-H33 & 0.95 \\
\hline C34-C35 & $1.379(3)$ & C34-H34 & 0.95 \\
\hline C35-C36 & $1.383(3)$ & C35-H35 & 0.95 \\
\hline
\end{tabular}




$\begin{array}{llll}\mathrm{C} 36-\mathrm{H} 36 & 0.95 & \mathrm{C} 37-\mathrm{C} 41 & 1.435(2) \\ \mathrm{C} 37-\mathrm{C} 38 & 1.436(2) & \mathrm{C} 38-\mathrm{C} 39 & 1.427(3) \\ \mathrm{C} 38-\mathrm{H} 38 & 1.0 & \mathrm{C} 39-\mathrm{C} 40 & 1.415(3) \\ \mathrm{C} 39-\mathrm{H} 39 & 1.0 & \mathrm{C} 40-\mathrm{C} 41 & 1.424(3) \\ \mathrm{C} 40-\mathrm{H} 40 & 1.0 & \mathrm{C} 41-\mathrm{H} 41 & 1.0 \\ \mathrm{C} 42-\mathrm{C} 43 & 1.434(2) & \mathrm{C} 42-\mathrm{C} 46 & 1.437(2) \\ \mathrm{C} 43-\mathrm{C} 44 & 1.418(3) & \mathrm{C} 43-\mathrm{H} 43 & 1.0 \\ \mathrm{C} 44-\mathrm{C} 45 & 1.416(3) & \mathrm{C} 44-\mathrm{H} 44 & 1.0 \\ \mathrm{C} 45-\mathrm{C} 46 & 1.414(3) & \mathrm{C} 45-\mathrm{H} 45 & 1.0 \\ \mathrm{C} 46-\mathrm{H} 46 & 1.0 & & \end{array}$

Table S8e. Bond angles $\left({ }^{\circ}\right)$ for $8 \mathrm{cb}$.

$\begin{array}{llll}\text { O1-Ni1-C1 } & 40.41(6) & \text { O1-Ni1-P1 } & 153.65(4) \\ \text { C1-Ni1-P1 } & 113.54(5) & \text { O1-Ni1-P2 } & 100.98(4) \\ \text { C1-Ni1-P2 } & 141.36(5) & \text { P1-Ni1-P2 } & 104.787(18) \\ \text { C42-Fe1-C46 } & 41.51(7) & \text { C42-Fe1-C38 } & 110.80(7) \\ \text { C46-Fe1-C38 } & 140.11(7) & \text { C42-Fe1-C37 } & 110.51(7) \\ \text { C46-Fe1-C37 } & 111.60(7) & \text { C38-Fe1-C37 } & 41.24(7) \\ \text { C42-Fe1-C41 } & 138.88(7) & \text { C46-Fe1-C41 } & 111.28(7) \\ \text { C38-Fe1-C41 } & 69.01(7) & \text { C37-Fe1-C41 } & 41.07(7) \\ \text { C42-Fe1-C43 } & 41.20(7) & \text { C46-Fe1-C43 } & 69.08(7) \\ \text { C38-Fe1-C43 } & 110.52(8) & \text { C37-Fe1-C43 } & 138.56(7) \\ \text { C41-Fe1-C43 } & 179.52(8) & \text { C42-Fe1-C39 } & 139.37(8) \\ \text { C46-Fe1-C39 } & 178.92(8) & \text { C38-Fe1-C39 } & 40.88(7) \\ \text { C37-Fe1-C39 } & 68.91(7) & \text { C41-Fe1-C39 } & 68.42(8) \\ \text { C43-Fe1-C39 } & 111.23(8) & \text { C42-Fe1-C45 } & 68.78(7) \\ \text { C46-Fe1-C45 } & 40.52(7) & \text { C38-Fe1-C45 } & 178.37(8) \\ \text { C37-Fe1-C45 } & 140.38(7) & \text { C41-Fe1-C45 } & 112.40(7) \\ \text { C43-Fe1-C45 } & 68.08(8) & \text { C39-Fe1-C45 } & 138.51(8) \\ \text { C42-Fe1-C40 } & 179.12(7) & \text { C46-Fe1-C40 } & 138.85(8)\end{array}$




\begin{tabular}{|c|c|c|c|}
\hline C38-Fe1-C40 & $68.44(8)$ & $\mathrm{C} 37-\mathrm{Fe} 1-\mathrm{C} 40$ & $68.65(7)$ \\
\hline $\mathrm{C} 41-\mathrm{Fe} 1-\mathrm{C} 40$ & $40.59(7)$ & $\mathrm{C} 43-\mathrm{Fe} 1-\mathrm{C} 40$ & $139.33(7)$ \\
\hline $\mathrm{C} 39-\mathrm{Fe} 1-\mathrm{C} 40$ & $40.28(8)$ & $\mathrm{C} 45-\mathrm{Fe} 1-\mathrm{C} 40$ & $112.00(8)$ \\
\hline $\mathrm{C} 42-\mathrm{Fe} 1-\mathrm{C} 44$ & $68.74(7)$ & $\mathrm{C} 46-\mathrm{Fe} 1-\mathrm{C} 44$ & $68.39(8)$ \\
\hline C38-Fe1-C44 & $138.13(8)$ & C37-Fe1-C44 & $178.93(8)$ \\
\hline C41-Fe1-C44 & $139.99(7)$ & $\mathrm{C} 43-\mathrm{Fe} 1-\mathrm{C} 44$ & $40.38(7)$ \\
\hline C39-Fe1-C44 & $111.12(8)$ & $\mathrm{C} 45-\mathrm{Fe} 1-\mathrm{C} 44$ & $40.25(8)$ \\
\hline $\mathrm{C} 40-\mathrm{Fe} 1-\mathrm{C} 44$ & 112.11(8) & $\mathrm{C} 42-\mathrm{P} 2-\mathrm{C} 25$ & $105.79(8)$ \\
\hline C42-P2-C31 & $103.33(8)$ & $\mathrm{C} 25-\mathrm{P} 2-\mathrm{C} 31$ & $100.25(8)$ \\
\hline C42-P2-Ni1 & $110.81(6)$ & C25-P2-Ni1 & $119.83(6)$ \\
\hline C31-P2-Ni1 & $115.06(6)$ & C37-P1-C13 & $101.40(8)$ \\
\hline C37-P1-C19 & $100.22(8)$ & C13-P1-C19 & $105.56(8)$ \\
\hline C37-P1-Ni1 & $118.10(6)$ & C13-P1-Ni1 & $113.29(6)$ \\
\hline C19-P1-Ni1 & $116.23(6)$ & C1-O1-Ni1 & $71.08(9)$ \\
\hline $\mathrm{O} 1-\mathrm{C} 1-\mathrm{C} 2$ & $135.19(16)$ & O1-C1-Ni1 & $68.51(9)$ \\
\hline C2-C1-Ni1 & 156.11(14) & $\mathrm{C} 1-\mathrm{C} 2-\mathrm{C} 7$ & $121.13(16)$ \\
\hline $\mathrm{C} 1-\mathrm{C} 2-\mathrm{C} 3$ & $119.26(16)$ & $\mathrm{C} 7-\mathrm{C} 2-\mathrm{C} 3$ & $119.59(15)$ \\
\hline $\mathrm{C} 2-\mathrm{C} 3-\mathrm{C} 4$ & $116.78(16)$ & $\mathrm{C} 2-\mathrm{C} 3-\mathrm{H} 3 \mathrm{~A}$ & 108.1 \\
\hline $\mathrm{C} 4-\mathrm{C} 3-\mathrm{H} 3 \mathrm{~A}$ & 108.1 & $\mathrm{C} 2-\mathrm{C} 3-\mathrm{H} 3 \mathrm{~B}$ & 108.1 \\
\hline C4-C3-H3B & 108.1 & $\mathrm{H} 3 \mathrm{~A}-\mathrm{C} 3-\mathrm{H} 3 \mathrm{~B}$ & 107.3 \\
\hline $\mathrm{C} 5-\mathrm{C} 4-\mathrm{C} 6$ & $111.0(2)$ & $\mathrm{C} 5-\mathrm{C} 4-\mathrm{C} 3$ & $112.01(17)$ \\
\hline C6-C4-C3 & $109.75(19)$ & C5-C4-H4 & 108.0 \\
\hline C6-C4-H4 & 108.0 & $\mathrm{C} 3-\mathrm{C} 4-\mathrm{H} 4$ & 108.0 \\
\hline C4-C5-H5A & 109.5 & C4-C5-H5B & 109.5 \\
\hline H5A-C5-H5B & 109.5 & $\mathrm{C} 4-\mathrm{C} 5-\mathrm{H} 5 \mathrm{C}$ & 109.5 \\
\hline H5A-C5-H5C & 109.5 & H5B-C5-H5C & 109.5 \\
\hline C4-C6-H6A & 109.5 & C4-C6-H6B & 109.5 \\
\hline H6A-C6-H6B & 109.5 & C4-C6-H6C & 109.5 \\
\hline H6A-C6-H6C & 109.5 & H6B-C6-H6C & 109.5 \\
\hline $\mathrm{C} 8-\mathrm{C} 7-\mathrm{C} 12$ & $115.42(16)$ & $\mathrm{C} 8-\mathrm{C} 7-\mathrm{C} 2$ & $122.65(16)$ \\
\hline
\end{tabular}




\begin{tabular}{|c|c|c|c|}
\hline $\mathrm{C} 12-\mathrm{C} 7-\mathrm{C} 2$ & $121.94(16)$ & $\mathrm{C} 9-\mathrm{C} 8-\mathrm{C} 7$ & $122.03(18)$ \\
\hline C9-C8-H8 & 119.0 & $\mathrm{C} 7-\mathrm{C} 8-\mathrm{H} 8$ & 119.0 \\
\hline $\mathrm{C} 10-\mathrm{C} 9-\mathrm{C} 8$ & $120.90(19)$ & C10-C9-H9 & 119.5 \\
\hline C8-C9-H9 & 119.5 & C11-C10-C9 & $118.60(19)$ \\
\hline C11-C10-H10 & 120.7 & C9-C10-H10 & 120.7 \\
\hline $\mathrm{C} 10-\mathrm{C} 11-\mathrm{C} 12$ & $120.67(19)$ & C10-C11-H11 & 119.7 \\
\hline C12-C11-H11 & 119.7 & $\mathrm{C} 11-\mathrm{C} 12-\mathrm{C} 7$ & $122.36(18)$ \\
\hline C11-C12-H12 & 118.8 & C7-C12-H12 & 118.8 \\
\hline C14-C13-C18 & $118.72(16)$ & C14-C13-P1 & $118.31(13)$ \\
\hline C18-C13-P1 & $122.81(14)$ & C15-C14-C13 & $120.67(17)$ \\
\hline C15-C14-H14 & 119.7 & C13-C14-H14 & 119.7 \\
\hline C16-C15-C14 & 119.81(19) & C16-C15-H15 & 120.1 \\
\hline C14-C15-H15 & 120.1 & $\mathrm{C} 17-\mathrm{C} 16-\mathrm{C} 15$ & $120.24(18)$ \\
\hline C17-C16-H16 & 119.9 & C15-C16-H16 & 119.9 \\
\hline C16-C17-C18 & $119.98(18)$ & C16-C17-H17 & 120.0 \\
\hline C18-C17-H17 & 120.0 & $\mathrm{C} 17-\mathrm{C} 18-\mathrm{C} 13$ & $120.57(18)$ \\
\hline C17-C18-H18 & 119.7 & C13-C18-H18 & 119.7 \\
\hline C20-C19-C24 & $119.34(17)$ & C20-C19-P1 & $123.25(14)$ \\
\hline C24-C19-P1 & $117.41(14)$ & $\mathrm{C} 19-\mathrm{C} 20-\mathrm{C} 21$ & $120.0(2)$ \\
\hline C19-C20-H20 & 120.0 & $\mathrm{C} 21-\mathrm{C} 20-\mathrm{H} 20$ & 120.0 \\
\hline $\mathrm{C} 22-\mathrm{C} 21-\mathrm{C} 20$ & $120.1(2)$ & $\mathrm{C} 22-\mathrm{C} 21-\mathrm{H} 21$ & 119.9 \\
\hline $\mathrm{C} 20-\mathrm{C} 21-\mathrm{H} 21$ & 119.9 & $\mathrm{C} 23-\mathrm{C} 22-\mathrm{C} 21$ & $119.9(2)$ \\
\hline $\mathrm{C} 23-\mathrm{C} 22-\mathrm{H} 22$ & 120.1 & $\mathrm{C} 21-\mathrm{C} 22-\mathrm{H} 22$ & 120.1 \\
\hline C22-C23-C24 & $120.4(2)$ & $\mathrm{C} 22-\mathrm{C} 23-\mathrm{H} 23$ & 119.8 \\
\hline $\mathrm{C} 24-\mathrm{C} 23-\mathrm{H} 23$ & 119.8 & C23-C24-C19 & $120.2(2)$ \\
\hline $\mathrm{C} 23-\mathrm{C} 24-\mathrm{H} 24$ & 119.9 & C19-C24-H24 & 119.9 \\
\hline $\mathrm{C} 30-\mathrm{C} 25-\mathrm{C} 26$ & $119.10(17)$ & $\mathrm{C} 30-\mathrm{C} 25-\mathrm{P} 2$ & $118.59(14)$ \\
\hline C26-C25-P2 & $122.27(13)$ & $\mathrm{C} 27-\mathrm{C} 26-\mathrm{C} 25$ & $120.10(18)$ \\
\hline C27-C26-H26 & 119.9 & $\mathrm{C} 25-\mathrm{C} 26-\mathrm{H} 26$ & 119.9 \\
\hline C26-C27-C28 & $120.33(19)$ & C26-C27-H27 & 119.8 \\
\hline
\end{tabular}




\begin{tabular}{|c|c|c|c|}
\hline $\mathrm{C} 28-\mathrm{C} 27-\mathrm{H} 27$ & 119.8 & $\mathrm{C} 29-\mathrm{C} 28-\mathrm{C} 27$ & $119.86(18)$ \\
\hline $\mathrm{C} 29-\mathrm{C} 28-\mathrm{H} 28$ & 120.1 & $\mathrm{C} 27-\mathrm{C} 28-\mathrm{H} 28$ & 120.1 \\
\hline C28-C29-C30 & $120.09(18)$ & $\mathrm{C} 28-\mathrm{C} 29-\mathrm{H} 29$ & 120.0 \\
\hline C30-C29-H29 & 120.0 & $\mathrm{C} 25-\mathrm{C} 30-\mathrm{C} 29$ & $120.51(18)$ \\
\hline C25-C30-H30 & 119.7 & C29-C30-H30 & 119.7 \\
\hline C32-C31-C36 & $118.80(17)$ & C32-C31-P2 & $122.61(14)$ \\
\hline C36-C31-P2 & $118.58(13)$ & C31-C32-C33 & $120.5(2)$ \\
\hline C31-C32-H32 & 119.8 & C33-C32-H32 & 119.8 \\
\hline C34-C33-C32 & $120.4(2)$ & C34-C33-H33 & 119.8 \\
\hline C32-C33-H33 & 119.8 & C33-C34-C35 & $119.60(18)$ \\
\hline C33-C34-H34 & 120.2 & C35-C34-H34 & 120.2 \\
\hline C34-C35-C36 & $120.45(19)$ & C34-C35-H35 & 119.8 \\
\hline C36-C35-H35 & 119.8 & C35-C36-C31 & $120.25(18)$ \\
\hline C35-C36-H36 & 119.9 & C31-C36-H36 & 119.9 \\
\hline C41-C37-C38 & $107.37(15)$ & C41-C37-P1 & $127.48(14)$ \\
\hline C38-C37-P1 & $125.14(14)$ & $\mathrm{C} 41-\mathrm{C} 37-\mathrm{Fe} 1$ & $69.69(10)$ \\
\hline C38-C37-Fe1 & $69.13(10)$ & $\mathrm{P} 1-\mathrm{C} 37-\mathrm{Fe} 1$ & $125.67(9)$ \\
\hline C39-C38-C37 & $107.96(16)$ & C39-C38-Fe1 & $70.13(10)$ \\
\hline C37-C38-Fe1 & $69.63(10)$ & C39-C38-H38 & 126.0 \\
\hline C37-C38-H38 & 126.0 & Fe1-C38-H38 & 126.0 \\
\hline C40-C39-C38 & $108.21(17)$ & $\mathrm{C} 40-\mathrm{C} 39-\mathrm{Fe} 1$ & $70.13(11)$ \\
\hline C38-C39-Fe1 & $69.00(10)$ & C40-C39-H39 & 125.9 \\
\hline C38-C39-H39 & 125.9 & Fe1-C39-H39 & 125.9 \\
\hline C39-C40-C41 & $108.54(17)$ & C39-C40-Fe1 & $69.59(11)$ \\
\hline C41-C40-Fe1 & $69.36(10)$ & C39-C40-H40 & 125.7 \\
\hline C41-C40-H40 & 125.7 & $\mathrm{Fe} 1-\mathrm{C} 40-\mathrm{H} 40$ & 125.7 \\
\hline $\mathrm{C} 40-\mathrm{C} 41-\mathrm{C} 37$ & 107.91(16) & $\mathrm{C} 40-\mathrm{C} 41-\mathrm{Fe} 1$ & $70.06(10)$ \\
\hline C37-C41-Fe1 & $69.24(10)$ & $\mathrm{C} 40-\mathrm{C} 41-\mathrm{H} 41$ & 126.0 \\
\hline C37-C41-H41 & 126.0 & Fe1-C41-H41 & 126.0 \\
\hline C43-C42-C46 & $107.26(15)$ & C43-C42-P2 & $129.81(14)$ \\
\hline
\end{tabular}




$\begin{array}{llll}\text { C46-C42-P2 } & 122.65(13) & \text { C43-C42-Fe1 } & 70.26(10) \\ \text { C46-C42-Fe1 } & 69.30(10) & \text { P2-C42-Fe1 } & 120.87(9) \\ \text { C44-C43-C42 } & 107.96(16) & \text { C44-C43-Fe1 } & 70.22(10) \\ \text { C42-C43-Fe1 } & 68.54(10) & \text { C44-C43-H43 } & 126.0 \\ \text { C42-C43-H43 } & 126.0 & \text { Fe1-C43-H43 } & 126.0 \\ \text { C45-C44-C43 } & 108.29(16) & \text { C45-C44-Fe1 } & 69.65(10) \\ \text { C43-C44-Fe1 } & 69.40(10) & \text { C45-C44-H44 } & 125.9 \\ \text { C43-C44-H44 } & 125.9 & \text { Fe1-C44-H44 } & 125.9 \\ \text { C46-C45-C44 } & 108.59(17) & \text { C46-C45-Fe1 } & 68.73(10) \\ \text { C44-C45-Fe1 } & 70.09(11) & \text { C46-C45-H45 } & 125.7 \\ \text { C44-C45-H45 } & 125.7 & \text { Fe1-C45-H45 } & 125.7 \\ \text { C45-C46-C42 } & 107.90(16) & \text { C45-C46-Fe1 } & 70.75(10) \\ \text { C42-C46-Fe1 } & 69.19(10) & \text { C45-C46-H46 } & 126.0 \\ \text { C42-C46-H46 } & 126.0 & \text { Fe1-C46-H46 } & 126.0\end{array}$

Table S8f. Torsion angles $\left({ }^{\circ}\right)$ for $8 \mathrm{cb}$.

$\begin{array}{llll}\text { P1-Ni1-O1-C1 } & 10.47(15) & \text { P2-Ni1-O1-C1 } & 178.26(9) \\ \text { Ni1-O1-C1-C2 } & -176.3(2) & \text { P1-Ni1-C1-O1 } & -174.95(7) \\ \text { P2-Ni1-C1-O1 } & -2.73(14) & \text { O1-Ni1-C1-C2 } & 173.6(4) \\ \text { P1-Ni1-C1-C2 } & -1.3(4) & \text { P2-Ni1-C1-C2 } & 170.9(3) \\ \text { O1-C1-C2-C7 } & 3.0(3) & \text { Ni1-C1-C2-C7 } & -168.5(3) \\ \text { O1-C1-C2-C3 } & -178.69(19) & \text { Ni1-C1-C2-C3 } & 9.8(4) \\ \text { C1-C2-C3-C4 } & 112.5(2) & \text { C7-C2-C3-C4 } & -69.2(2) \\ \text { C2-C3-C4-C5 } & -58.3(2) & \text { C2-C3-C4-C6 } & 177.97(18) \\ \text { C1-C2-C7-C8 } & -1.5(3) & \text { C3-C2-C7-C8 } & -179.78(17) \\ \text { C1-C2-C7-C12 } & 178.68(17) & \text { C3-C2-C7-C12 } & 0.4(3) \\ \text { C12-C7-C8-C9 } & 0.5(3) & \text { C2-C7-C8-C9 } & -179.32(19) \\ \text { C7-C8-C9-C10 } & 0.5(3) & \text { C8-C9-C10-C11 } & -0.9(3) \\ \text { C9-C10-C11-C12 } & 0.3(3) & \text { C10-C11-C12-C7 } & 0.8(3) \\ \text { C8-C7-C12-C11 } & -1.1(3) & \text { C2-C7-C12-C11 } & 178.69(18) \\ & & & \text { S148 }\end{array}$




\begin{tabular}{|c|c|c|c|}
\hline C37-P1-C13-C14 & $146.68(14)$ & C19-P1-C13-C14 & $-109.19(15)$ \\
\hline Ni1-P1-C13-C14 & $19.09(16)$ & C37-P1-C13-C18 & $-28.68(16)$ \\
\hline C19-P1-C13-C18 & $75.45(16)$ & Ni1-P1-C13-C18 & $-156.27(13)$ \\
\hline C18-C13-C14-C15 & $0.6(3)$ & $\mathrm{P} 1-\mathrm{C} 13-\mathrm{C} 14-\mathrm{C} 15$ & $-174.97(15)$ \\
\hline $\mathrm{C} 13-\mathrm{C} 14-\mathrm{C} 15-\mathrm{C} 16$ & $-0.1(3)$ & $\mathrm{C} 14-\mathrm{C} 15-\mathrm{C} 16-\mathrm{C} 17$ & $-0.7(3)$ \\
\hline $\mathrm{C} 15-\mathrm{C} 16-\mathrm{C} 17-\mathrm{C} 18$ & $0.8(3)$ & C16-C17-C18-C13 & $-0.3(3)$ \\
\hline C14-C13-C18-C17 & $-0.4(3)$ & $\mathrm{P} 1-\mathrm{C} 13-\mathrm{C} 18-\mathrm{C} 17$ & $174.92(14)$ \\
\hline C37-P1-C19-C20 & $97.63(17)$ & C13-P1-C19-C20 & $-7.37(18)$ \\
\hline Ni1-P1-C19-C20 & $-133.87(15)$ & C37-P1-C19-C24 & $-82.80(15)$ \\
\hline C13-P1-C19-C24 & $172.20(14)$ & Ni1-P1-C19-C24 & $45.69(16)$ \\
\hline C24-C19-C20-C21 & $-1.0(3)$ & $\mathrm{P} 1-\mathrm{C} 19-\mathrm{C} 20-\mathrm{C} 21$ & $178.51(18)$ \\
\hline $\mathrm{C} 19-\mathrm{C} 20-\mathrm{C} 21-\mathrm{C} 22$ & $1.5(4)$ & $\mathrm{C} 20-\mathrm{C} 21-\mathrm{C} 22-\mathrm{C} 23$ & $-0.3(4)$ \\
\hline $\mathrm{C} 21-\mathrm{C} 22-\mathrm{C} 23-\mathrm{C} 24$ & $-1.3(4)$ & C22-C23-C24-C19 & $1.7(3)$ \\
\hline C20-C19-C24-C23 & $-0.5(3)$ & $\mathrm{P} 1-\mathrm{C} 19-\mathrm{C} 24-\mathrm{C} 23$ & 179.8 \\
\hline C42-P2-C25-C30 & $-119.05(15)$ & $\mathrm{C} 31-\mathrm{P} 2-\mathrm{C} 25-\mathrm{C} 30$ & $133.82(15)$ \\
\hline Ni1-P2-C25-C30 & $6.95(17)$ & C42-P2-C25-C26 & $63.11(17)$ \\
\hline C31-P2-C25-C26 & $-44.03(17)$ & Ni1-P2-C25-C26 & $-170.89(13)$ \\
\hline $\mathrm{C} 30-\mathrm{C} 25-\mathrm{C} 26-\mathrm{C} 27$ & $1.2(3)$ & P2-C25-C26-C27 & $179.07(15)$ \\
\hline $\mathrm{C} 25-\mathrm{C} 26-\mathrm{C} 27-\mathrm{C} 28$ & $-0.5(3)$ & C26-C27-C28-C29 & $0.0(3)$ \\
\hline $\mathrm{C} 27-\mathrm{C} 28-\mathrm{C} 29-\mathrm{C} 30$ & $-0.2(3)$ & $\mathrm{C} 26-\mathrm{C} 25-\mathrm{C} 30-\mathrm{C} 29$ & $-1.5(3)$ \\
\hline P2-C25-C30-C29 & $-179.38(15)$ & $\mathrm{C} 28-\mathrm{C} 29-\mathrm{C} 30-\mathrm{C} 25$ & $1.0(3)$ \\
\hline C42-P2-C31-C32 & $12.33(19)$ & C25-P2-C31-C32 & $121.42(17)$ \\
\hline Ni1-P2-C31-C32 & $-108.60(17)$ & C42-P2-C31-C36 & $-166.63(14)$ \\
\hline C25-P2-C31-C36 & $-57.54(16)$ & Ni1-P2-C31-C36 & $72.44(15)$ \\
\hline C36-C31-C32-C33 & $0.5(3)$ & P2-C31-C32-C33 & $-178.45(18)$ \\
\hline C31-C32-C33-C34 & $0.1(4)$ & С32-C33-С34-C35 & $-0.5(4)$ \\
\hline C33-C34-C35-C36 & $0.2(3)$ & C34-C35-C36-C31 & $0.4(3)$ \\
\hline C32-C31-C36-C35 & $-0.8(3)$ & P2-C31-C36-C35 & $178.22(15)$ \\
\hline C13-P1-C37-C41 & $133.41(15)$ & C19-P1-C37-C41 & $25.08(16)$ \\
\hline Ni1-P1-C37-C41 & $-102.19(15)$ & C13-P1-C37-C38 & -47.68( \\
\hline
\end{tabular}




$\begin{array}{lllll}\text { C19-P1-C37-C38 } & -156.01(15) & \text { Ni1-P1-C37-C38 } & 76.72(15) \\ \text { C13-P1-C37-Fe1 } & -135.90(11) & \text { C19-P1-C37-Fe1 } & 115.77(11) \\ \text { Ni1-P1-C37-Fe1 } & -11.50(13) & \text { C41-C37-C38-C39 } & -0.46(19) \\ \text { P1-C37-C38-C39 } & -179.55(13) & \text { Fe1-C37-C38-C39 } & -59.90(12) \\ \text { C41-C37-C38-Fe1 } & 59.44(11) & \text { P1-C37-C38-Fe1 } & -119.65(13) \\ \text { C37-C38-C39-C40 } & 0.2(2) & \text { Fe1-C38-C39-C40 } & -59.36(13) \\ \text { C37-C38-C39-Fe1 } & 59.59(12) & \text { C38-C39-C40-C41 } & 0.1(2) \\ \text { Fe1-C39-C40-C41 } & -58.56(12) & \text { C38-C39-C40-Fe1 } & 58.66(12) \\ \text { C39-C40-C41-C37 } & -0.4(2) & \text { Fe1-C40-C41-C37 } & -59.09(12) \\ \text { C39-C40-C41-Fe1 } & 58.70(13) & \text { C38-C37-C41-C40 } & 0.52(19) \\ \text { P1-C37-C41-C40 } & 179.59(13) & \text { Fe1-C37-C41-C40 } & 59.60(12) \\ \text { C38-C37-C41-Fe1 } & -59.08(11) & \text { P1-C37-C41-Fe1 } & 119.98(14) \\ \text { C25-P2-C42-C43 } & -13.21(18) & \text { C31-P2-C42-C43 } & 91.69(17) \\ \text { Ni1-P2-C42-C43 } & -144.54(14) & \text { C25-P2-C42-C46 } & 159.90(14) \\ \text { C31-P2-C42-C46 } & -95.20(15) & \text { Ni1-P2-C42-C46 } & 28.57(15) \\ \text { C25-P2-C42-Fe1 } & 75.99(11) & \text { C31-P2-C42-Fe1 } & -179.11(10) \\ \text { Ni1-P2-C42-Fe1 } & -55.34(11) & \text { C46-C42-C43-C44 } & -0.32(19) \\ \text { P2-C42-C43-C44 } & 173.61(13) & \text { Fe1-C42-C43-C44 } & 59.36(12) \\ \text { C46-C42-C43-Fe1 } & -59.69(11) & \text { P2-C42-C43-Fe1 } & 114.24(14) \\ \text { C42-C43-C44-C45 } & 0.7(2) & \text { Fe1-C43-C44-C45 } & 58.97(13) \\ \text { C42-C43-C44-Fe1 } & -58.32(12) & \text { C43-C44-C45-C46 } & -0.7(2) \\ \text { Fe1-C44-C45-C46 } & 58.07(12) & \text { C43-C44-C45-Fe1 } & -58.81(13) \\ \text { C44-C45-C46-C42 } & 0.5(2) & \text { Fe1-C45-C46-C42 } & 59.45(11) \\ \text { C4-C46-Fe1 } & -58.91(13) & \text { C43-C42-C46-C45 } & -0.13(19) \\ \text { C4-Fe1 } & 60.30(11) & \text { P2-C42-C46-Fe1 } & -114.16(12)\end{array}$

\section{Table S8g. Anisotropic atomic displacement parameters $\left(\AA^{2}\right)$ for $8 \mathrm{cb}$.}

The anisotropic atomic displacement factor exponent takes the form: $-2 \pi^{2}$ [ $\left.\mathrm{h}^{2} \mathrm{a}^{* 2} \mathrm{U}_{11}+\ldots+2 \mathrm{~h} \mathrm{k} \mathrm{a}^{*} \mathrm{~b}^{*} \mathrm{U}_{12}\right]$ 


\begin{tabular}{|c|c|c|c|c|c|c|}
\hline & $\mathbf{U}_{11}$ & $\mathbf{U}_{22}$ & $\mathbf{U}_{33}$ & $\mathbf{U}_{23}$ & $\mathbf{U}_{13}$ & $\mathbf{U}_{12}$ \\
\hline $\mathrm{Ni1}$ & $0.01442(10)$ & $0.01785(11)$ & $0.01629(11)$ & $-0.00127(8)$ & $0.00511(8)$ & $-0.00012(8)$ \\
\hline $\mathrm{e} 1$ & $0.01948(12)$ & $0.02296(13)$ & $0.01613(12)$ & $-0.00187(9)$ & $00562(10)$ & $-0.00261(9)$ \\
\hline 2 & $0.01633(19)$ & $0.0174(2)$ & $0.0183(2)$ & $-0.00124(16)$ & $0.00528(16)$ & $-0.00090(1$ \\
\hline 1 & $0.01435(19)$ & $0.0191(2)$ & $0.0169(2)$ & $-0.00171(16)$ & $0.00461(16)$ & $-0.00050(1$ \\
\hline 01 & $0.0190(6)$ & $0.0215(6)$ & $0.0234(6)$ & $-0.0033(5)$ & $0.0109(5)$ & $-0.0018(5)$ \\
\hline $\mathrm{C} 1$ & $0.0180(8)$ & $0.0212(8)$ & $0.0195(8)$ & $0.0016(7)$ & $0.0070(7)$ & $0.0012(6)$ \\
\hline $\mathrm{C} 2$ & $0.0225(8)$ & $0.0209(8)$ & $0.0195(8)$ & $-0.0013(7)$ & $0.0073(7)$ & $0.0001(7)$ \\
\hline $\mathrm{C} 3$ & $0.0274(C$ & $0.0269(9)$ & $0.0258(9)$ & $-0.0068(8)$ & $0.0126(8)$ & $-0.0053(7)$ \\
\hline $\mathrm{C} 4$ & $0.0247(9)$ & $0.0372(11)$ & $0.0357(11)$ & $-0.0114(9)$ & $0.0100(8)$ & $-0.0053(8)$ \\
\hline $\mathrm{C} 5$ & $0.0399(13)$ & $0.0503(15)$ & $0.0560(16)$ & $-0.0081(12)$ & $-0.0067(11)$ & $0.0070(11)$ \\
\hline C6 & $0.0374(12)$ & $0.0664(17)$ & $0.0574(16)$ & $-0.0259(14)$ & $0.0133(11)$ & $-0.0229(12)$ \\
\hline 67 & $0.0225(8)$ & $0.0217(9)$ & $0.0195(8)$ & $0.0009(7)$ & $0.0059(7)$ & $0.0022(7)$ \\
\hline $\mathrm{C} 8$ & $0.0251(9)$ & $0.0348(11)$ & $0.0330(11)$ & $-0.0124(9)$ & $0.0127(8)$ & $-0.0025(8)$ \\
\hline C9 & $0.0234(9)$ & $0.0463(13)$ & $0.0429(12)$ & $-0.0134(10)$ & $0.0145(9)$ & $-0.0041(9)$ \\
\hline $\mathrm{C} 10$ & $0.0266(10)$ & $0.0457(13)$ & $0.0386(12)$ & $-0.0086(10)$ & $0.0145(9)$ & $0.0068(9)$ \\
\hline $\mathrm{C} 11$ & $0.0349(10)$ & $0.0304(10)$ & $0.0322(11)$ & $-0.0089(8)$ & $0.0103(9)$ & $0.0055(8)$ \\
\hline $\mathrm{C} 12$ & $0.0266(9)$ & $0.0230(9)$ & $0.0282(10)$ & $-0.0014(7)$ & $0.0086(8)$ & $0.0011(7)$ \\
\hline $\mathrm{C} 13$ & $0.0155(7)$ & $0.0196(8)$ & 0.0194 & -0.0028 & $0.0030(6)$ & $0.0001(6)$ \\
\hline C14 & $0.0182(8)$ & $0.0330(10)$ & $0.0222(9)$ & $-0.0006(8)$ & $0.0058(7)$ & $-0.0001(7)$ \\
\hline $\mathrm{C} 15$ & $0.0259(9)$ & $0.0431(12)$ & $0.0260(10)$ & $0.0074(9)$ & $0.0010(8)$ & $0.0030(8)$ \\
\hline C16 & $0.0217(9)$ & 0.03 & 0.0 & 0.0 & ) & $0.0086(8)$ \\
\hline $\mathrm{C} 17$ & $0.0178(8)$ & $0.0319(10)$ & $0.0382(11)$ & -0.0092 & $0.0072(8)$ & $0.0014(7)$ \\
\hline $\mathrm{C} 18$ & $0.0192(8)$ & $0.0268(9)$ & $0.0257(9)$ & $-0.0047(7)$ & $0.0070(7)$ & $-0.0012(7)$ \\
\hline $\mathrm{C} 19$ & $0.0243(8)$ & $0.0198(8)$ & $0.0205(9)$ & -0.0004 & $0.0099(7)$ & $-0.0002(7)$ \\
\hline $\mathrm{C} 20$ & $0.0253(9)$ & $0.0279(10$ & 0.0524( & $-0.0074(9)$ & $0.0168(9)$ & $-0.0042(8)$ \\
\hline $\mathrm{C} 21$ & $0.0444(13)$ & $0.0321(12$ & 0.081 & -0.012 & 0.0348 & $-0.0155(10)$ \\
\hline $\mathrm{C} 22$ & $0.0692(16)$ & $0.0216(10)$ & $0.0654(16)$ & $-0.0009(10)$ & $0.0453(14)$ & $-0.0065(11)$ \\
\hline $\mathrm{C} 23$ & $0.0634(15)$ & $0.0267(11)$ & $0.0353(12)$ & $0.0089(9)$ & $0.0185(11)$ & $0.0138(10)$ \\
\hline $\mathrm{C} 24$ & $0.0504(10)$ & $0.0 \angle 80(10)$ & $0.0247(10$ & -0.0003 & 0.0052 & .0047 \\
\hline
\end{tabular}




\begin{tabular}{|c|c|c|c|c|c|c|}
\hline & $\mathrm{U}_{11}$ & $\mathbf{U}_{22}$ & $\mathbf{U}_{33}$ & $\mathbf{U}_{23}$ & $\mathbf{U}_{13}$ & $\mathbf{U}_{12}$ \\
\hline $\mathrm{C} 25$ & $0.0222(8)$ & $0.0192(8)$ & $0.0208(9)$ & $0.0004(7)$ & $0.0089(7)$ & $0.0007(6)$ \\
\hline $\mathrm{C} 26$ & $0.0279(9)$ & $0.0242(9)$ & $0.0263(10)$ & $-0.0004(7)$ & $0.0087(8)$ & $-0.0008(7)$ \\
\hline $\mathrm{C} 27$ & $0.0444(11)$ & $0.0219(9)$ & $0.0311(11)$ & $-0.0022(8)$ & $0.0159(9)$ & $0.0024(8)$ \\
\hline $\mathrm{C} 28$ & $0.0403(11)$ & $0.0264(10)$ & $0.0366(11)$ & $0.0072(8)$ & $0.0181(9)$ & $0.0121(8)$ \\
\hline $\mathrm{C} 29$ & $0.0242(9)$ & $0.0339(11)$ & $0.0364(11)$ & $0.0097(9)$ & $0.0076(8)$ & $0.0064(8)$ \\
\hline $\mathrm{C} 30$ & $0.0235(9)$ & $0.0235(9)$ & $0.0272(10)$ & $0.0019(7)$ & $0.0052(7)$ & $-0.0009(7)$ \\
\hline $\mathrm{C} 31$ & $0.0186(8)$ & $0.0180(8)$ & $0.0255(9)$ & $-0.0028(7)$ & $0.0076(7)$ & $-0.0015(6)$ \\
\hline $\mathrm{C} 32$ & $0.0229(9)$ & $0.0481(13)$ & $0.0333(11)$ & $0.0113(10)$ & $0.0050(8)$ & $-0.0059(9)$ \\
\hline $\mathrm{C} 33$ & $0.0194(9)$ & $0.0590(15)$ & $0.0446(13)$ & $0.0074(11)$ & $0.0023(9)$ & $-0.0090(9)$ \\
\hline $\mathrm{C} 34$ & $0.0261(10)$ & $0.0367(11)$ & $0.0481(13)$ & $-0.0040(10)$ & $0.0171(9)$ & $-0.0108(8)$ \\
\hline C35 & $0.0362(11)$ & $0.0293(10)$ & $0.0365(11)$ & $0.0018(8)$ & $0.0199(9)$ & $-0.0035(8)$ \\
\hline C36 & $0.0257(9)$ & $0.0288(10)$ & $0.0240(9)$ & $0.0000(8)$ & $0.0087(7)$ & $-0.0017(7)$ \\
\hline C37 & $0.0154(7)$ & $0.0245(9)$ & $0.0194(8)$ & $-0.0011(7)$ & $0.0048(6)$ & $-0.0022(6)$ \\
\hline C38 & $0.0191(8)$ & $0.0273(9)$ & $0.0239(9)$ & $-0.0034(7)$ & $0.0076(7)$ & $0.0003(7)$ \\
\hline C39 & $0.0243(9)$ & $0.0347(10)$ & $0.0266(10)$ & $-0.0066(8)$ & $0.0123(8)$ & $-0.0023(8)$ \\
\hline C40 & $0.0249(9)$ & $0.0375(11)$ & $0.0219(9)$ & $0.0001(8)$ & $0.0108(7)$ & $-0.0072(8)$ \\
\hline C41 & $0.0209(8)$ & $0.0265(9)$ & $0.0224(9)$ & $0.0009(7)$ & $0.0075(7)$ & $-0.0048(7)$ \\
\hline C42 & $0.0178(8)$ & $0.0214(8)$ & $0.0199(8)$ & $-0.0029(7)$ & $0.0060(7)$ & $-0.0046(6)$ \\
\hline $\mathrm{C} 43$ & $0.0243(8)$ & $0.0258(9)$ & $0.0222(9)$ & $-0.0067(7)$ & $0.0065(7)$ & $-0.0062(7)$ \\
\hline C44 & $0.0277(9)$ & $0.0332(10)$ & $0.0188(9)$ & $-0.0040(8)$ & $0.0036(7)$ & $-0.0070(8)$ \\
\hline C45 & $0.0223(9)$ & $0.0307(10)$ & $0.0222(9)$ & $0.0043(7)$ & $0.0000(7)$ & $-0.0039(7)$ \\
\hline $\mathrm{C} 4 \mathrm{C}$ & $0.0158(8)$ & $0.0230(9)$ & $0.0231(9)$ & $-0.0003(7)$ & $0.0036(7)$ & $-0.0011(6)$ \\
\hline
\end{tabular}

Table S8h. Hydrogen atomic coordinates and isotropic atomic displacement parameters $\left(\AA^{2}\right)$ for

$8 c b$.

$\begin{array}{lllll} & \mathbf{x} / \mathbf{a} & \mathbf{y} / \mathbf{b} & \mathbf{z} / \mathbf{c} & \mathbf{U}(\mathbf{e q}) \\ \text { H3A } & 0.2741 & 0.5406 & 0.3268 & 0.031 \\ \text { H3B } & 0.3529 & 0.4808 & 0.3034 & 0.031\end{array}$




\begin{tabular}{|c|c|c|c|c|}
\hline & $\mathbf{x} / \mathbf{a}$ & $\mathbf{y} / \mathbf{b}$ & $\mathbf{z} / \mathbf{c}$ & $\mathbf{U}(\mathbf{e q})$ \\
\hline $\mathrm{H} 4$ & 0.2835 & 0.5146 & 0.1719 & 0.039 \\
\hline $\mathrm{H} 5 \mathrm{~A}$ & 0.1369 & 0.5997 & 0.1283 & 0.08 \\
\hline H5B & 0.2580 & 0.6302 & 0.1935 & 0.08 \\
\hline $\mathrm{H} 5 \mathrm{C}$ & 0.1269 & 0.6133 & 0.2139 & 0.08 \\
\hline H6A & 0.0676 & 0.4968 & 0.2313 & 0.081 \\
\hline H6B & 0.1558 & 0.4367 & 0.2168 & 0.081 \\
\hline $\mathrm{H} 6 \mathrm{C}$ & 0.0691 & 0.4791 & 0.1448 & 0.081 \\
\hline H8 & 0.6732 & 0.6306 & 0.3220 & 0.036 \\
\hline H9 & 0.8351 & 0.6038 & 0.2670 & 0.044 \\
\hline $\mathrm{H} 10$ & 0.8133 & 0.5125 & 0.1834 & 0.043 \\
\hline H11 & 0.6276 & 0.4464 & 0.1585 & 0.039 \\
\hline H12 & 0.4668 & 0.4712 & 0.2147 & 0.031 \\
\hline H14 & 0.1644 & 0.7069 & 0.3099 & 0.029 \\
\hline H15 & -0.0173 & 0.7631 & 0.2328 & 0.04 \\
\hline H16 & -0.2021 & 0.7726 & 0.2741 & 0.042 \\
\hline H17 & -0.2072 & 0.7243 & 0.3908 & 0.035 \\
\hline H18 & -0.0255 & 0.6685 & 0.4685 & 0.029 \\
\hline $\mathrm{H} 20$ & 0.0173 & 0.5540 & 0.3956 & 0.041 \\
\hline $\mathrm{H} 21$ & -0.0048 & 0.4375 & 0.3901 & 0.059 \\
\hline $\mathrm{H} 22$ & 0.1729 & 0.3693 & 0.4489 & 0.056 \\
\hline $\mathrm{H} 23$ & 0.3717 & 0.4172 & 0.5118 & 0.049 \\
\hline $\mathrm{H} 24$ & 0.3975 & 0.5331 & 0.5126 & 0.037 \\
\hline H26 & 0.5163 & 0.9021 & 0.5729 & 0.031 \\
\hline $\mathrm{H} 27$ & 0.3875 & 0.9978 & 0.5540 & 0.038 \\
\hline $\mathrm{H} 28$ & 0.1791 & 0.9963 & 0.4668 & 0.04 \\
\hline $\mathrm{H} 29$ & 0.0990 & 0.8986 & 0.3990 & 0.038 \\
\hline $\mathrm{H} 30$ & 0.2277 & 0.8026 & 0.4169 & 0.03 \\
\hline H32 & 0.7478 & 0.7675 & 0.6101 & 0.043 \\
\hline H33 & 0.9403 & 0.8154 & 0.6024 & 0.051 \\
\hline
\end{tabular}




$\begin{array}{lllll} & \mathbf{x} / \mathbf{a} & \mathbf{y} / \mathbf{b} & \mathbf{z} / \mathbf{c} & \mathbf{U}(\mathbf{e q}) \\ \text { H34 } & 0.9438 & 0.8776 & 0.4940 & 0.043 \\ \text { H35 } & 0.7545 & 0.8908 & 0.3919 & 0.038 \\ \text { H36 } & 0.5617 & 0.8425 & 0.3983 & 0.031 \\ \text { H38 } & 0.1721 & 0.7686 & 0.5488 & 0.028 \\ \text { H39 } & 0.1762 & 0.7570 & 0.6935 & 0.033 \\ \text { H40 } & 0.2388 & 0.6353 & 0.7369 & 0.033 \\ \text { H41 } & 0.2741 & 0.5699 & 0.6201 & 0.028 \\ \text { H43 } & 0.4681 & 0.8265 & 0.6727 & 0.029 \\ \text { H44 } & 0.5131 & 0.7465 & 0.7909 & 0.033 \\ \text { H45 } & 0.5751 & 0.6307 & 0.7487 & 0.032 \\ \text { H46 } & 0.5677 & 0.6372 & 0.6040 & 0.025\end{array}$


Table S9a. Sample and crystal data for 8cc.

\begin{tabular}{|c|c|c|}
\hline Identification code & \multicolumn{2}{|l|}{$8 \mathrm{cc}$} \\
\hline Chemical formula & \multicolumn{2}{|l|}{$\mathrm{C}_{56} \mathrm{H}_{53} \mathrm{FeNiOP}_{2}$} \\
\hline Formula weight & \multicolumn{2}{|l|}{$918.48 \mathrm{~g} / \mathrm{mol}$} \\
\hline Temperature & \multicolumn{2}{|l|}{$150(1) \mathrm{K}$} \\
\hline Wavelength & \multicolumn{2}{|l|}{$0.71073 \AA$} \\
\hline Crystal size & \multicolumn{2}{|c|}{$0.210 \times 0.392 \times 0.392 \mathrm{~mm}$} \\
\hline Crystal habit & \multicolumn{2}{|c|}{ clear intense orange prism } \\
\hline Crystal system & \multicolumn{2}{|l|}{ monoclinic } \\
\hline Space group & \multicolumn{2}{|l|}{ P $121 / \mathrm{c} 1$} \\
\hline \multirow[t]{3}{*}{ Unit cell dimensions } & $\mathrm{a}=15.7448(5) \AA$ & $\alpha=90^{\circ}$ \\
\hline & $\mathrm{b}=16.7714(5) \AA$ & $\beta=93.8920(10)^{\circ}$ \\
\hline & $\mathrm{c}=17.6503(5) \AA$ & $\gamma=90^{\circ}$ \\
\hline Volume & \multicolumn{2}{|l|}{$4650.0(2) \AA^{3}$} \\
\hline $\mathbf{Z}$ & \multicolumn{2}{|l|}{4} \\
\hline Density (calculated) & \multicolumn{2}{|l|}{$1.312 \mathrm{~g} / \mathrm{cm}^{3}$} \\
\hline Absorption coefficient & \multicolumn{2}{|l|}{$0.825 \mathrm{~mm}^{-1}$} \\
\hline $\mathbf{F}(000)$ & \\
\hline
\end{tabular}

Table S9b. Data collection and structure refinement for $8 \mathrm{cc}$.

Theta range for data collection $\quad 2.07$ to $27.88^{\circ}$

Index ranges $\quad-20<=\mathrm{h}<=20,-22<=\mathrm{k}<=22,-23<=1<=18$

Reflections collected $\quad 132703$

Independent reflections $\quad 11079[\mathrm{R}(\mathrm{int})=0.0348]$

Coverage of independent reflections $100.0 \%$

Absorption correction multi-scan

Max. and min. transmission $\quad 0.8460$ and 0.7380

Structure solution technique direct methods

Structure solution program $\quad$ SHELXT-2014/9 (Sheldrick, 2014) 


\begin{tabular}{|c|c|}
\hline Refinement method & Full-matrix least-squares on $\mathrm{F}^{2}$ \\
\hline Refinement program & SHELXL-2014/6 (Sheldrick, 2014) \\
\hline Function minimized & $\Sigma \mathrm{w}\left(\mathrm{F}_{\mathrm{o}}^{2}-\mathrm{F}_{\mathrm{c}}^{2}\right)^{2}$ \\
\hline Data / restraints / parameters & $11079 / 0$ / 541 \\
\hline Goodness-of-fit on $F^{2}$ & 1.038 \\
\hline$\Delta / \sigma_{\max }$ & 0.002 \\
\hline \multirow[t]{2}{*}{ Final $\mathbf{R}$ indices } & 9111 data; $\mathrm{I}>2 \sigma(\mathrm{I}) \mathrm{R} 1=0.0370, \mathrm{wR} 2=0.0938$ \\
\hline & $\mathrm{R} 1=0.0495, \mathrm{wR} 2=0.1022$ \\
\hline Weighting scheme & $\begin{array}{l}\mathrm{w}=1 /\left[\sigma^{2}\left(\mathrm{~F}_{\mathrm{o}}^{2}\right)+(0.0481 \mathrm{P})^{2}+4.3928 \mathrm{P}\right] \\
\text { where } \mathrm{P}=\left(\mathrm{F}_{\mathrm{o}}{ }^{2}+2 \mathrm{~F}_{\mathrm{c}}{ }^{2}\right) / 3\end{array}$ \\
\hline Largest diff. peak and hole & 0.706 and $-0.550 \mathrm{e}^{-3}$ \\
\hline R.M.S. deviation from mean & $0.064 \mathrm{e} \AA^{-3}$ \\
\hline
\end{tabular}

Table S9c. Atomic coordinates and equivalent isotropic atomic displacement parameters $\left(\AA^{2}\right)$ for

8cc.

$\mathrm{U}(\mathrm{eq})$ is defined as one third of the trace of the orthogonalized $\mathrm{U}_{\mathrm{ij}}$ tensor.

$\begin{array}{lllll} & \mathbf{x} / \mathbf{a} & \mathbf{y} / \mathbf{b} & \mathbf{z} / \mathbf{c} & \mathbf{U}(\mathbf{e q}) \\ \mathrm{Ni1} & 0.31548(2) & 0.64114(2) & 0.64290(2) & 0.01963(7) \\ \mathrm{Fe} 1 & 0.31436(2) & 0.78940(2) & 0.45494(2) & 0.02418(8) \\ \mathrm{P} 1 & 0.20755(3) & 0.66057(3) & 0.56311(3) & 0.02098(10) \\ \mathrm{P} 2 & 0.40760(3) & 0.73866(3) & 0.62355(3) & 0.01946(10) \\ \text { O1 } & 0.36433(8) & 0.59103(8) & 0.72998(7) & 0.0256(3) \\ \mathrm{C} 1 & 0.29419(12) & 0.55571(11) & 0.70771(10) & 0.0221(4) \\ \mathrm{C} 2 & 0.24615(12) & 0.49731(11) & 0.73556(11) & 0.0238(4) \\ \mathrm{C} 3 & 0.17030(13) & 0.46510(12) & 0.68908(12) & 0.0296(4) \\ \mathrm{C} 4 & 0.17696(16) & 0.37612(13) & 0.66870(13) & 0.0380(5) \\ \mathrm{C} 5 & 0.2568(2) & 0.35799(16) & 0.62918(17) & 0.0529(7) \\ \mathrm{C} 6 & 0.0981(2) & 0.35033(18) & 0.6203(2) & 0.0722(10)\end{array}$


$\begin{array}{llll}\mathbf{x} / \mathbf{a} & \mathbf{y} / \mathbf{b} & \mathbf{z} / \mathbf{c} & \mathrm{U}(\mathrm{eq})\end{array}$

C36 0.42541(16) 0.95735(14) 0.72881(14) 0.0410(5)

C37 0.44286(14) 0.89098(13) 0.68535(12) 0.0314(4)

C38 0.20825(12) 0.74222(12) 0.49544(11) 0.0250(4)

C39 0.20298(14) 0.73813(15) 0.41396(12) 0.0349(5)

C40 0.20890(14) 0.81674(16) 0.38540(13) 0.0411(6)

C41 0.21670(14) 0.87055(15) 0.44790(15) 0.0393(5)

C42 0.21600(13) 0.82484(12) 0.51568(13) 0.0296(4)

C43 0.41667(11) 0.76792(11) 0.52672(10) 0.0205(4)

C44 0.41190(12) 0.71026(12) 0.46616(11) 0.0251(4)

C45 0.41602(13) 0.75180(14) 0.39650(11) 0.0305(4)

C46 0.42263(13) 0.83430(13) 0.41246(11) 0.0306(4)

C47 0.42339(13) 0.84526(12) 0.49267(11) 0.0261(4)

C48 0.03811(19) 0.11357(17) 0.59295(16) 0.0898(13)

C49 0.11630(18) 0.11405(15) $0.63455(18)$ 0.0846(11)

C50 0.14730(16) 0.04456(19) 0.66929(18) 0.1079(16)

$\begin{array}{lllll}\text { C51 0.1001(2) } & 0.97458(16) & 0.6624(2) & 0.1198(19)\end{array}$

C52 0.02191(19) 0.97409(17) 0.6208(3)

C53 0.99091(16) 0.0436(2) $0.5861(2) \quad 0.133(2)$

C54 0.55596(19) 0.56342(18) 0.49866(18) 0.0567(8)

$\begin{array}{llllll}\text { C55 0.5318(2) } & 0.52290(18) & 0.43221(18) & 0.0568(8)\end{array}$

C56 0.4761(2) $\quad 0.45989(17) 0.43403(18) 0.0572(8)$

Table S9d. Bond lengths ( $(\AA)$ for 8cc.

$\begin{array}{llll}\text { Ni1-O1 } & 1.8708(13) & \text { Ni1-C1 } & 1.8777(19) \\ \text { Ni1-P1 } & 2.1568(5) & \text { Ni1-P2 } & 2.2277(5) \\ \text { Fe1-C43 } & 2.0132(18) & \text { Fe1-C38 } & 2.022(2) \\ \text { Fe1-C47 } & 2.028(2) & \text { Fe1-C44 } & 2.0293(19) \\ \text { Fe1-C42 } & 2.031(2) & \text { Fe1-C39 } & 2.041(2)\end{array}$




$\begin{array}{llll}\text { Fe1-C40 } & 2.049(2) & \text { Fe1-C41 } & 2.051(2) \\ \text { Fe1-C46 } & 2.052(2) & \text { Fe1-C45 } & 2.061(2) \\ \text { P1-C38 } & 1.818(2) & \text { P1-C14 } & 1.826(2) \\ \text { P1-C20 } & 1.836(2) & \text { P2-C43 } & 1.7932(18) \\ \text { P2-C32 } & 1.8269(19) & \text { P2-C26 } & 1.8276(19) \\ \text { O1-C1 } & 1.291(2) & \text { C1-C2 } & 1.350(3) \\ \text { C2-C7 } & 1.477(3) & \text { C2-C3 } & 1.503(3) \\ \text { C3-C4 } & 1.540(3) & \text { C3-H3A } & 0.99 \\ \text { C3-H3B } & 0.99 & \text { C4-C5 } & 1.510(4) \\ \text { C4-C6 } & 1.522(3) & \text { C4-H4 } & 1.0 \\ \text { C5-H5A } & 0.98 & \text { C5-H5B } & 0.98 \\ \text { C5-H5C } & 0.98 & \text { C6-H6A } & 0.98 \\ \text { C6-H6B } & 0.98 & \text { C6-H6C } & 0.98 \\ \text { C7-C8 } & 1.397(3) & \text { C7-C12 } & 1.402(3) \\ \text { C8-C9 } & 1.389(3) & \text { C8-H8 } & 0.95 \\ \text { C9-C10 } & 1.387(3) & \text { C9-H9 } & 0.95 \\ \text { C10-C11 } & 1.384(3) & \text { C10-C13 } & 1.510(3) \\ \text { C11-C12 } & 1.391(3) & \text { C11-H11 } & 0.95 \\ \text { C12-H12 } & 0.95 & \text { C13-H13A } & 0.98 \\ \text { C13-H13B } & 0.98 & \text { C13-H13C } & 0.98 \\ \text { C14-C15 } & 1.380(3) & \text { C14-C19 } & 1.385(3) \\ \text { C15-C16 } & 1.388(3) & \text { C15-H15 } & 0.95 \\ \text { C16-C17 } & 1.376(4) & \text { C16-H16 } & 0.95 \\ \text { C17-C18 } & 1.366(4) & \text { C17-H17 } & 0.95 \\ \text { C18-C19 } & 1.395(4) & \text { C18-H18 } & 0.95 \\ \text { C20 } & 0.9535 & \text { C24-C25 } & 1.386(3)\end{array}$




\begin{tabular}{|c|c|c|c|}
\hline $\mathrm{C} 24-\mathrm{H} 24$ & 0.95 & $\mathrm{C} 25-\mathrm{H} 25$ & 0.95 \\
\hline $\mathrm{C} 26-\mathrm{C} 27$ & $1.388(3)$ & C26-C31 & $1.398(3)$ \\
\hline $\mathrm{C} 27-\mathrm{C} 28$ & $1.386(3)$ & $\mathrm{C} 27-\mathrm{H} 27$ & 0.95 \\
\hline C28-C29 & $1.380(3)$ & C28-H28 & 0.95 \\
\hline C29-C30 & $1.380(3)$ & С29-H29 & 0.95 \\
\hline C30-C31 & $1.384(3)$ & C30-H30 & 0.95 \\
\hline C31-H31 & 0.95 & C32-C33 & $1.391(3)$ \\
\hline C32-C37 & $1.392(3)$ & C33-C34 & $1.388(3)$ \\
\hline C33-H33 & 0.95 & C34-C35 & $1.379(4)$ \\
\hline C34-H34 & 0.95 & $\mathrm{C} 35-\mathrm{C} 36$ & $1.379(3)$ \\
\hline C35-H35 & 0.95 & C36-C37 & $1.390(3)$ \\
\hline C36-H36 & 0.95 & C37-H37 & 0.95 \\
\hline $\mathrm{C} 38-\mathrm{C} 42$ & $1.434(3)$ & C38-C39 & $1.437(3)$ \\
\hline C39-C40 & $1.417(3)$ & C39-H39 & 1.0 \\
\hline $\mathrm{C} 40-\mathrm{C} 41$ & $1.424(4)$ & $\mathrm{C} 40-\mathrm{H} 40$ & 1.0 \\
\hline $\mathrm{C} 41-\mathrm{C} 42$ & $1.422(3)$ & C41-H41 & 1.0 \\
\hline C42-H42 & 1.0 & $\mathrm{C} 43-\mathrm{C} 47$ & $1.436(3)$ \\
\hline $\mathrm{C} 43-\mathrm{C} 44$ & $1.440(3)$ & $\mathrm{C} 44-\mathrm{C} 45$ & $1.418(3)$ \\
\hline C44-H44 & 1.0 & $\mathrm{C} 45-\mathrm{C} 46$ & $1.414(3)$ \\
\hline $\mathrm{C} 45-\mathrm{H} 45$ & 1.0 & $\mathrm{C} 46-\mathrm{C} 47$ & $1.427(3)$ \\
\hline C46-H46 & 1.0 & C47-H47 & 1.0 \\
\hline C48-C49 & 1.39 & $\mathrm{C} 48-\mathrm{C} 53$ & 1.39 \\
\hline $\mathrm{C} 48-\mathrm{H} 48$ & 0.95 & C49-C50 & 1.39 \\
\hline C49-H49 & 0.95 & C50-C51 & 1.39 \\
\hline C50-H50 & 0.95 & C51-C52 & 1.39 \\
\hline C51-H51 & 0.95 & C52-C53 & 1.39 \\
\hline C52-H52 & 0.95 & C53-H53 & 0.95 \\
\hline C54-C56 & $1.379(5)$ & C54-C55 & $1.386(4)$ \\
\hline C54-H54 & 0.95 & C55-C56 & $1.375(5)$ \\
\hline C55-H55 & 0.95 & C56-C54 & $1.378(5)$ \\
\hline
\end{tabular}


C56-H56 $\quad 0.95$

Table S9e. Bond angles $\left({ }^{\circ}\right)$ for $8 \mathrm{cc}$.

\begin{tabular}{|c|c|c|c|}
\hline O1-Ni1-C1 & $40.28(7)$ & O1-Ni1-P1 & $150.00(4)$ \\
\hline C1-Ni1-P1 & $110.35(6)$ & O1-Ni1-P2 & $102.71(4)$ \\
\hline C1-Ni1-P2 & $143.00(6)$ & P1-Ni1-P2 & $106.34(2)$ \\
\hline C43-Fe1-C38 & $110.64(7)$ & C43-Fe1-C47 & $41.64(7)$ \\
\hline C38-Fe1-C47 & $139.98(8)$ & C43-Fe1-C44 & $41.73(8)$ \\
\hline C38-Fe1-C44 & $110.33(8)$ & C47-Fe1-C44 & $69.64(8)$ \\
\hline $\mathrm{C} 43-\mathrm{Fe} 1-\mathrm{C} 42$ & $109.05(8)$ & C38-Fe1-C42 & $41.44(8)$ \\
\hline C47-Fe1-C42 & $110.50(9)$ & C44-Fe1-C42 & $137.64(8)$ \\
\hline C43-Fe1-C39 & $141.19(8)$ & C38-Fe1-C39 & $41.41(8)$ \\
\hline C47-Fe1-C39 & $177.16(9)$ & C44-Fe1-C39 & $112.66(9)$ \\
\hline C42-Fe1-C39 & $69.08(9)$ & $\mathrm{C} 43-\mathrm{Fe} 1-\mathrm{C} 40$ & $176.82(10)$ \\
\hline C38-Fe1-C40 & $69.15(8)$ & C47-Fe1-C40 & $136.63(9)$ \\
\hline $\mathrm{C} 44-\mathrm{Fe} 1-\mathrm{C} 40$ & $141.45(10)$ & $\mathrm{C} 42-\mathrm{Fe} 1-\mathrm{C} 40$ & $68.58(9)$ \\
\hline C39-Fe1-C40 & $40.54(10)$ & C43-Fe1-C41 & $136.18(10)$ \\
\hline C38-Fe1-C41 & $69.34(8)$ & C47-Fe1-C41 & 109.13(9) \\
\hline C44-Fe1-C41 & $177.80(9)$ & C42-Fe1-C41 & $40.76(9)$ \\
\hline C39-Fe1-C41 & $68.63(10)$ & C40-Fe1-C41 & $40.66(11)$ \\
\hline C43-Fe1-C46 & $69.29(8)$ & C38-Fe1-C46 & 178.41(9) \\
\hline C47-Fe1-C46 & $40.94(8)$ & C44-Fe1-C46 & $68.50(8)$ \\
\hline C42-Fe1-C46 & $140.14(9)$ & C39-Fe1-C46 & $137.76(9)$ \\
\hline C40-Fe1-C46 & $111.02(9)$ & C41-Fe1-C46 & $111.87(9)$ \\
\hline C43-Fe1-C45 & $69.19(8)$ & C38-Fe1-C45 & $138.19(9)$ \\
\hline $\mathrm{C} 47-\mathrm{Fe} 1-\mathrm{C} 45$ & $68.71(9)$ & C44-Fe1-C45 & $40.57(8)$ \\
\hline $\mathrm{C} 42-\mathrm{Fe} 1-\mathrm{C} 45$ & $178.12(8)$ & C39-Fe1-C45 & $111.81(9)$ \\
\hline $\mathrm{C} 40-\mathrm{Fe} 1-\mathrm{C} 45$ & $113.20(9)$ & C41-Fe1-C45 & $141.01(9)$ \\
\hline C46-Fe1-C45 & $40.23(9)$ & C38-P1-C14 & $100.60(9)$ \\
\hline C38-P1-C20 & $99.44(9)$ & C14-P1-C20 & 105.99(9) \\
\hline & & & S161 \\
\hline
\end{tabular}




\begin{tabular}{|c|c|c|c|}
\hline C38-P1-Ni1 & $120.21(7)$ & C14-P1-Ni1 & $115.24(7)$ \\
\hline C20-P1-Ni1 & $113.17(7)$ & $\mathrm{C} 43-\mathrm{P} 2-\mathrm{C} 32$ & $106.82(9)$ \\
\hline C43-P2-C26 & $102.17(9)$ & C32-P2-C26 & $100.33(9)$ \\
\hline C43-P2-Ni1 & $116.22(6)$ & C32-P2-Ni1 & $112.06(7)$ \\
\hline C26-P2-Ni1 & $117.49(6)$ & C1-O1-Ni1 & $70.15(10)$ \\
\hline $\mathrm{O} 1-\mathrm{C} 1-\mathrm{C} 2$ & $135.30(17)$ & O1-C1-Ni1 & $69.57(10)$ \\
\hline C2-C1-Ni1 & $154.53(15)$ & $\mathrm{C} 1-\mathrm{C} 2-\mathrm{C} 7$ & $119.85(17)$ \\
\hline $\mathrm{C} 1-\mathrm{C} 2-\mathrm{C} 3$ & $120.40(17)$ & $\mathrm{C} 7-\mathrm{C} 2-\mathrm{C} 3$ & $119.75(17)$ \\
\hline $\mathrm{C} 2-\mathrm{C} 3-\mathrm{C} 4$ & $114.30(18)$ & $\mathrm{C} 2-\mathrm{C} 3-\mathrm{H} 3 \mathrm{~A}$ & 108.7 \\
\hline $\mathrm{C} 4-\mathrm{C} 3-\mathrm{H} 3 \mathrm{~A}$ & 108.7 & $\mathrm{C} 2-\mathrm{C} 3-\mathrm{H} 3 \mathrm{~B}$ & 108.7 \\
\hline C4-C3-H3B & 108.7 & H3A-C3-H3B & 107.6 \\
\hline C5-C4-C6 & $110.9(2)$ & $\mathrm{C} 5-\mathrm{C} 4-\mathrm{C} 3$ & $112.0(2)$ \\
\hline C6-C4-C3 & $109.9(2)$ & C5-C4-H4 & 107.9 \\
\hline C6-C4-H4 & 107.9 & $\mathrm{C} 3-\mathrm{C} 4-\mathrm{H} 4$ & 107.9 \\
\hline C4-C5-H5A & 109.5 & C4-C5-H5B & 109.5 \\
\hline H5A-C5-H5B & 109.5 & $\mathrm{C} 4-\mathrm{C} 5-\mathrm{H} 5 \mathrm{C}$ & 109.5 \\
\hline H5A-C5-H5C & 109.5 & H5B-C5-H5C & 109.5 \\
\hline C4-C6-H6A & 109.5 & C4-C6-H6B & 109.5 \\
\hline H6А-C6-H6B & 109.5 & C4-C6-H6C & 109.5 \\
\hline H6A-C6-H6C & 109.5 & H6B-C6-H6C & 109.5 \\
\hline $\mathrm{C} 8-\mathrm{C} 7-\mathrm{C} 12$ & $116.40(18)$ & $\mathrm{C} 8-\mathrm{C} 7-\mathrm{C} 2$ & $122.03(17)$ \\
\hline $\mathrm{C} 12-\mathrm{C} 7-\mathrm{C} 2$ & $121.48(18)$ & $\mathrm{C} 9-\mathrm{C} 8-\mathrm{C} 7$ & $121.68(19)$ \\
\hline C9-C8-H8 & 119.2 & $\mathrm{C} 7-\mathrm{C} 8-\mathrm{H} 8$ & 119.2 \\
\hline C10-C9-C8 & $121.6(2)$ & C10-C9-H9 & 119.2 \\
\hline C8-C9-H9 & 119.2 & C11-C10-C9 & $117.19(19)$ \\
\hline C11-C10-C13 & $122.2(2)$ & C9-C10-C13 & $120.6(2)$ \\
\hline $\mathrm{C} 10-\mathrm{C} 11-\mathrm{C} 12$ & $121.8(2)$ & C10-C11-H11 & 119.1 \\
\hline C12-C11-H11 & 119.1 & $\mathrm{C} 11-\mathrm{C} 12-\mathrm{C} 7$ & $121.3(2)$ \\
\hline C11-C12-H12 & 119.3 & C7-C12-H12 & 119.3 \\
\hline C10-C13-H13A & 109.5 & C10-C13-H13B & 109.5 \\
\hline
\end{tabular}




\begin{tabular}{|c|c|c|c|}
\hline H13A-C13-H13B & 109.5 & $\mathrm{C} 10-\mathrm{C} 13-\mathrm{H} 13 \mathrm{C}$ & 109.5 \\
\hline H13A-C13-H13C & 109.5 & H13B-C13-H13C & 109.5 \\
\hline C15-C14-C19 & $118.8(2)$ & C15-C14-P1 & $117.88(15)$ \\
\hline C19-C14-P1 & $123.30(17)$ & C14-C15-C16 & $120.8(2)$ \\
\hline C14-C15-H15 & 119.6 & C16-C15-H15 & 119.6 \\
\hline C17-C16-C15 & $120.0(2)$ & C17-C16-H16 & 120.0 \\
\hline C15-C16-H16 & 120.0 & C18-C17-C16 & $119.8(2)$ \\
\hline C18-C17-H17 & 120.1 & $\mathrm{C} 16-\mathrm{C} 17-\mathrm{H} 17$ & 120.1 \\
\hline C17-C18-C19 & $120.6(2)$ & C17-C18-H18 & 119.7 \\
\hline C19-C18-H18 & 119.7 & C14-C19-C18 & $120.1(2)$ \\
\hline C14-C19-H19 & 120.0 & C18-C19-H19 & 120.0 \\
\hline $\mathrm{C} 21-\mathrm{C} 20-\mathrm{C} 25$ & $118.7(2)$ & $\mathrm{C} 21-\mathrm{C} 20-\mathrm{P} 1$ & $119.58(16)$ \\
\hline $\mathrm{C} 25-\mathrm{C} 20-\mathrm{P} 1$ & $121.66(17)$ & $\mathrm{C} 20-\mathrm{C} 21-\mathrm{C} 22$ & $120.7(2)$ \\
\hline $\mathrm{C} 20-\mathrm{C} 21-\mathrm{H} 21$ & 119.6 & $\mathrm{C} 22-\mathrm{C} 21-\mathrm{H} 21$ & 119.6 \\
\hline C23-C22-C21 & $120.0(2)$ & $\mathrm{C} 23-\mathrm{C} 22-\mathrm{H} 22$ & 120.0 \\
\hline $\mathrm{C} 21-\mathrm{C} 22-\mathrm{H} 22$ & 120.0 & $\mathrm{C} 24-\mathrm{C} 23-\mathrm{C} 22$ & $119.8(2)$ \\
\hline $\mathrm{C} 24-\mathrm{C} 23-\mathrm{H} 23$ & 120.1 & $\mathrm{C} 22-\mathrm{C} 23-\mathrm{H} 23$ & 120.1 \\
\hline $\mathrm{C} 23-\mathrm{C} 24-\mathrm{C} 25$ & $120.4(2)$ & $\mathrm{C} 23-\mathrm{C} 24-\mathrm{H} 24$ & 119.8 \\
\hline $\mathrm{C} 25-\mathrm{C} 24-\mathrm{H} 24$ & 119.8 & $\mathrm{C} 24-\mathrm{C} 25-\mathrm{C} 20$ & $120.3(2)$ \\
\hline $\mathrm{C} 24-\mathrm{C} 25-\mathrm{H} 25$ & 119.9 & $\mathrm{C} 20-\mathrm{C} 25-\mathrm{H} 25$ & 119.9 \\
\hline C27-C26-C31 & $118.72(18)$ & $\mathrm{C} 27-\mathrm{C} 26-\mathrm{P} 2$ & $122.75(14)$ \\
\hline C31-C26-P2 & $118.53(15)$ & $\mathrm{C} 28-\mathrm{C} 27-\mathrm{C} 26$ & $120.78(19)$ \\
\hline $\mathrm{C} 28-\mathrm{C} 27-\mathrm{H} 27$ & 119.6 & $\mathrm{C} 26-\mathrm{C} 27-\mathrm{H} 27$ & 119.6 \\
\hline C29-C28-C27 & $120.0(2)$ & C29-C28-H28 & 120.0 \\
\hline $\mathrm{C} 27-\mathrm{C} 28-\mathrm{H} 28$ & 120.0 & $\mathrm{C} 28-\mathrm{C} 29-\mathrm{C} 30$ & $119.9(2)$ \\
\hline C28-C29-H29 & 120.1 & C30-C29-H29 & 120.1 \\
\hline C29-C30-C31 & $120.5(2)$ & C29-C30-H30 & 119.8 \\
\hline C31-C30-H30 & 119.8 & $\mathrm{C} 30-\mathrm{C} 31-\mathrm{C} 26$ & $120.1(2)$ \\
\hline $\mathrm{C} 30-\mathrm{C} 31-\mathrm{H} 31$ & 119.9 & C26-C31-H31 & 119.9 \\
\hline C33-C32-C37 & $119.00(18)$ & C33-C32-P2 & $119.22(15)$ \\
\hline
\end{tabular}




\begin{tabular}{|c|c|c|c|}
\hline C37-C32-P2 & $121.63(15)$ & C34-C33-C32 & $120.2(2)$ \\
\hline C34-C33-H33 & 119.9 & C32-C33-H33 & 119.9 \\
\hline C35-C34-C33 & $120.1(2)$ & C35-C34-H34 & 119.9 \\
\hline C33-C34-H34 & 119.9 & C36-C35-C34 & $120.3(2)$ \\
\hline C36-C35-H35 & 119.8 & C34-C35-H35 & 119.8 \\
\hline C35-C36-C37 & $119.7(2)$ & $\mathrm{C} 35-\mathrm{C} 36-\mathrm{H} 36$ & 120.1 \\
\hline C37-C36-H36 & 120.1 & C36-C37-C32 & $120.6(2)$ \\
\hline C36-C37-H37 & 119.7 & C32-C37-H37 & 119.7 \\
\hline C42-C38-C39 & $107.08(18)$ & C42-C38-P1 & $124.64(15)$ \\
\hline C39-C38-P1 & $128.27(17)$ & $\mathrm{C} 42-\mathrm{C} 38-\mathrm{Fe} 1$ & $69.62(12)$ \\
\hline C39-C38-Fe1 & $69.99(12)$ & $\mathrm{P} 1-\mathrm{C} 38-\mathrm{Fe} 1$ & $124.69(10)$ \\
\hline C40-C39-C38 & $108.1(2)$ & $\mathrm{C} 40-\mathrm{C} 39-\mathrm{Fe} 1$ & $70.02(13)$ \\
\hline C38-C39-Fe1 & $68.60(12)$ & C40-C39-H39 & 125.9 \\
\hline C38-C39-H39 & 125.9 & Fe1-C39-H39 & 125.9 \\
\hline C39-C40-C41 & $108.57(19)$ & $\mathrm{C} 39-\mathrm{C} 40-\mathrm{Fe} 1$ & $69.44(12)$ \\
\hline $\mathrm{C} 41-\mathrm{C} 40-\mathrm{Fe} 1$ & $69.75(12)$ & C39-C40-H40 & 125.7 \\
\hline C41-C40-H40 & 125.7 & Fe1-C40-H40 & 125.7 \\
\hline C42-C41-C40 & $107.8(2)$ & $\mathrm{C} 42-\mathrm{C} 41-\mathrm{Fe} 1$ & $68.89(12)$ \\
\hline C40-C41-Fe1 & $69.59(13)$ & C42-C41-H41 & 126.1 \\
\hline C40-C41-H41 & 126.1 & Fe1-C41-H41 & 126.1 \\
\hline C41-C42-C38 & $108.5(2)$ & $\mathrm{C} 41-\mathrm{C} 42-\mathrm{Fe} 1$ & $70.36(13)$ \\
\hline C38-C42-Fe1 & $68.95(12)$ & $\mathrm{C} 41-\mathrm{C} 42-\mathrm{H} 42$ & 125.8 \\
\hline C38-C42-H42 & 125.8 & $\mathrm{Fe} 1-\mathrm{C} 42-\mathrm{H} 42$ & 125.8 \\
\hline C47-C43-C44 & 107.31(16) & C47-C43-P2 & $131.21(15)$ \\
\hline C44-C43-P2 & $121.35(14)$ & $\mathrm{C} 47-\mathrm{C} 43-\mathrm{Fe} 1$ & $69.73(11)$ \\
\hline $\mathrm{C} 44-\mathrm{C} 43-\mathrm{Fe} 1$ & $69.74(10)$ & $\mathrm{P} 2-\mathrm{C} 43-\mathrm{Fe} 1$ & $122.46(10)$ \\
\hline C45-C44-C43 & $108.09(18)$ & $\mathrm{C} 45-\mathrm{C} 44-\mathrm{Fe} 1$ & $70.92(12)$ \\
\hline $\mathrm{C} 43-\mathrm{C} 44-\mathrm{Fe} 1$ & $68.54(10)$ & C45-C44-H44 & 126.0 \\
\hline C43-C44-H44 & 126.0 & Fe1-C44-H44 & 126.0 \\
\hline C46-C45-C44 & $108.36(18)$ & $\mathrm{C} 46-\mathrm{C} 45-\mathrm{Fe} 1$ & $69.53(12)$ \\
\hline
\end{tabular}




\begin{tabular}{|c|c|c|c|}
\hline $\mathrm{C} 44-\mathrm{C} 45-\mathrm{Fe} 1$ & $68.51(11)$ & $\mathrm{C} 46-\mathrm{C} 45-\mathrm{H} 45$ & 125.8 \\
\hline $\mathrm{C} 44-\mathrm{C} 45-\mathrm{H} 45$ & 125.8 & $\mathrm{Fe} 1-\mathrm{C} 45-\mathrm{H} 45$ & 125.8 \\
\hline $\mathrm{C} 45-\mathrm{C} 46-\mathrm{C} 47$ & $108.62(17)$ & $\mathrm{C} 45-\mathrm{C} 46-\mathrm{Fe} 1$ & $70.24(12)$ \\
\hline $\mathrm{C} 47-\mathrm{C} 46-\mathrm{Fe} 1$ & $68.64(11)$ & $\mathrm{C} 45-\mathrm{C} 46-\mathrm{H} 46$ & 125.7 \\
\hline C47-C46-H46 & 125.7 & $\mathrm{Fe} 1-\mathrm{C} 46-\mathrm{H} 46$ & 125.7 \\
\hline $\mathrm{C} 46-\mathrm{C} 47-\mathrm{C} 43$ & $107.63(18)$ & $\mathrm{C} 46-\mathrm{C} 47-\mathrm{Fe} 1$ & $70.42(12)$ \\
\hline $\mathrm{C} 43-\mathrm{C} 47-\mathrm{Fe} 1$ & $68.62(11)$ & $\mathrm{C} 46-\mathrm{C} 47-\mathrm{H} 47$ & 126.2 \\
\hline C43-C47-H47 & 126.2 & Fe1-C47-H47 & 126.2 \\
\hline C49-C48-C53 & 120.0 & C49-C48-H48 & 120.0 \\
\hline C53-C48-H48 & 120.0 & C50-C49-C48 & 120.0 \\
\hline C50-C49-H49 & 120.0 & C48-C49-H49 & 120.0 \\
\hline C49-C50-C51 & 120.0 & C49-C50-H50 & 120.0 \\
\hline C51-C50-H50 & 120.0 & C52-C51-C50 & 120.0 \\
\hline C52-C51-H51 & 120.0 & C50-C51-H51 & 120.0 \\
\hline C51-C52-C53 & 120.0 & C51-C52-H52 & 120.0 \\
\hline C53-C52-H52 & 120.0 & C52-C53-C48 & 120.0 \\
\hline C52-C53-H53 & 120.0 & C48-C53-H53 & 120.0 \\
\hline C56-C54-C55 & $119.6(3)$ & C56-C54-H54 & 120.2 \\
\hline C55-C54-H54 & 120.2 & C56-C55-C54 & 119.7(3) \\
\hline C56-C55-H55 & 120.1 & C54-C55-H55 & 120.1 \\
\hline C55-C56-C54 & $120.6(3)$ & C55-C56-H56 & 119.7 \\
\hline С54-C56-H56 & 119.7 & & \\
\hline
\end{tabular}

Table S9f. Torsion angles $\left({ }^{\circ}\right)$ for $8 \mathrm{cc}$.

$\begin{array}{llll}\text { P1-Ni1-O1-C1 } & 14.50(16) & \text { P2-Ni1-O1-C1 } & 179.89(10) \\ \text { Ni1-O1-C1-C2 } & -173.3(3) & \text { P1-Ni1-C1-O1 } & -172.33(9) \\ \text { P2-Ni1-C1-O1 } & -0.17(16) & \text { O1-Ni1-C1-C2 } & 169.0(4) \\ \text { P1-Ni1-C1-C2 } & -3.3(4) & \text { P2-Ni1-C1-C2 } & 168.8(3) \\ \text { O1-C1-C2-C7 } & 6.2(4) & \text { Ni1-C1-C2-C7 } & -159.1(3) \\ \text { O1-C1-C2-C3 } & -174.0(2) & \text { Ni1-C1-C2-C3 } & 20.7(5) \\ & & & \mathrm{S} 165\end{array}$




\begin{tabular}{|c|c|c|c|}
\hline $\mathrm{C} 1-\mathrm{C} 2-\mathrm{C} 3-\mathrm{C} 4$ & $117.3(2)$ & $\mathrm{C} 7-\mathrm{C} 2-\mathrm{C} 3-\mathrm{C} 4$ & $-62.9(3)$ \\
\hline $\mathrm{C} 2-\mathrm{C} 3-\mathrm{C} 4-\mathrm{C} 5$ & $-54.9(3)$ & $\mathrm{C} 2-\mathrm{C} 3-\mathrm{C} 4-\mathrm{C} 6$ & $-178.7(2)$ \\
\hline $\mathrm{C} 1-\mathrm{C} 2-\mathrm{C} 7-\mathrm{C} 8$ & $-20.8(3)$ & $\mathrm{C} 3-\mathrm{C} 2-\mathrm{C} 7-\mathrm{C} 8$ & $159.40(19)$ \\
\hline $\mathrm{C} 1-\mathrm{C} 2-\mathrm{C} 7-\mathrm{C} 12$ & $155.6(2)$ & $\mathrm{C} 3-\mathrm{C} 2-\mathrm{C} 7-\mathrm{C} 12$ & $-24.2(3)$ \\
\hline $\mathrm{C} 12-\mathrm{C} 7-\mathrm{C} 8-\mathrm{C} 9$ & $-1.9(3)$ & $\mathrm{C} 2-\mathrm{C} 7-\mathrm{C} 8-\mathrm{C} 9$ & $174.75(19)$ \\
\hline C7-C8-C9-C10 & $0.2(3)$ & C8-C9-C10-C11 & $1.2(3)$ \\
\hline C8-C9-C10-C13 & $-179.8(2)$ & C9-C10-C11-C12 & $-0.9(3)$ \\
\hline C13-C10-C11-C12 & $-179.9(2)$ & $\mathrm{C} 10-\mathrm{C} 11-\mathrm{C} 12-\mathrm{C} 7$ & $-0.8(3)$ \\
\hline C8-C7-C12-C11 & $2.1(3)$ & $\mathrm{C} 2-\mathrm{C} 7-\mathrm{C} 12-\mathrm{C} 11$ & $-174.49(19)$ \\
\hline C38-P1-C14-C15 & $99.25(17)$ & C20-P1-C14-C15 & $-157.61(16)$ \\
\hline Ni1-P1-C14-C15 & $-31.61(18)$ & C38-P1-C14-C19 & $-78.2(2)$ \\
\hline C20-P1-C14-C19 & $24.9(2)$ & Ni1-P1-C14-C19 & $150.9(2)$ \\
\hline C19-C14-C15-C16 & $1.1(3)$ & P1-C14-C15-C16 & $-176.54(17)$ \\
\hline C14-C15-C16-C17 & $-0.4(3)$ & C15-C16-C17-C18 & $-0.5(4)$ \\
\hline C16-C17-C18-C19 & $0.7(5)$ & C15-C14-C19-C18 & $-0.9(4)$ \\
\hline P1-C14-C19-C18 & $176.6(2)$ & C17-C18-C19-C14 & $0.0(5)$ \\
\hline C38-P1-C20-C21 & $-149.47(18)$ & C14-P1-C20-C21 & $106.54(18)$ \\
\hline Ni1-P1-C20-C21 & $-20.71(19)$ & C38-P1-C20-C25 & $27.9(2)$ \\
\hline C14-P1-C20-C25 & $-76.1(2)$ & Ni1-P1-C20-C25 & $156.64(17)$ \\
\hline $\mathrm{C} 25-\mathrm{C} 20-\mathrm{C} 21-\mathrm{C} 22$ & $-1.1(4)$ & $\mathrm{P} 1-\mathrm{C} 20-\mathrm{C} 21-\mathrm{C} 22$ & $176.3(2)$ \\
\hline $\mathrm{C} 20-\mathrm{C} 21-\mathrm{C} 22-\mathrm{C} 23$ & $0.9(4)$ & $\mathrm{C} 21-\mathrm{C} 22-\mathrm{C} 23-\mathrm{C} 24$ & $0.0(4)$ \\
\hline $\mathrm{C} 22-\mathrm{C} 23-\mathrm{C} 24-\mathrm{C} 25$ & $-0.7(4)$ & $\mathrm{C} 23-\mathrm{C} 24-\mathrm{C} 25-\mathrm{C} 20$ & $0.4(4)$ \\
\hline C21-C20-C25-C24 & $0.5(4)$ & $\mathrm{P} 1-\mathrm{C} 20-\mathrm{C} 25-\mathrm{C} 24$ & $-176.9(2)$ \\
\hline C43-P2-C26-C27 & $7.89(18)$ & C32-P2-C26-C27 & $-102.00(17)$ \\
\hline Ni1-P2-C26-C27 & $136.32(15)$ & C43-P2-C26-C31 & $-172.81(15)$ \\
\hline C32-P2-C26-C31 & $77.30(16)$ & Ni1-P2-C26-C31 & $-44.38(17)$ \\
\hline C31-C26-C27-C28 & $-0.3(3)$ & $\mathrm{P} 2-\mathrm{C} 26-\mathrm{C} 27-\mathrm{C} 28$ & $178.99(16)$ \\
\hline $\mathrm{C} 26-\mathrm{C} 27-\mathrm{C} 28-\mathrm{C} 29$ & $-0.4(3)$ & $\mathrm{C} 27-\mathrm{C} 28-\mathrm{C} 29-\mathrm{C} 30$ & $0.8(4)$ \\
\hline C28-C29-C30-C31 & $-0.5(4)$ & $\mathrm{C} 29-\mathrm{C} 30-\mathrm{C} 31-\mathrm{C} 26$ & $-0.2(3)$ \\
\hline $\mathrm{C} 27-\mathrm{C} 26-\mathrm{C} 31-\mathrm{C} 30$ & $0.6(3)$ & P2-C26-C31-C30 & $-178.76(17)$ \\
\hline
\end{tabular}




\begin{tabular}{|c|c|c|c|}
\hline C43-P2-C32-C33 & $119.12(16)$ & C26-P2-C32-C33 & $-134.68(16)$ \\
\hline Ni1-P2-C32-C33 & $-9.23(17)$ & C43-P2-C32-C37 & $-65.32(18)$ \\
\hline C26-P2-C32-C37 & $40.87(18)$ & Ni1-P2-C32-C37 & $166.33(15)$ \\
\hline C37-C32-C33-C34 & $0.5(3)$ & P2-C32-C33-C34 & $176.19(17)$ \\
\hline C32-C33-C34-C35 & $0.6(4)$ & C33-C34-C35-C36 & $-1.1(4)$ \\
\hline C34-C35-C36-C37 & $0.4(4)$ & C35-C36-C37-C32 & $0.8(4)$ \\
\hline C33-C32-C37-C36 & $-1.2(3)$ & P2-C32-C37-C36 & $7(18)$ \\
\hline C14-P1-C38-C42 & $172.06(17)$ & C20-P1-C38-C42 & $63.68(18)$ \\
\hline Ni1-P1-C38-C42 & $-60.27(19)$ & C14-P1-C38-C39 & $-9.1(2)$ \\
\hline C20-P1-C38-C39 & $-117.51(19)$ & Ni1-P1-C38-C39 & $118.54(17)$ \\
\hline C14-P1-C38-Fe1 & $-100.04(13)$ & C20-P1-C38-Fe1 & $151.58(12)$ \\
\hline Ni1-P1-C38-Fe1 & $27.63(15)$ & C42-C38-C39-C40 & $1.0(2)$ \\
\hline P1-C38-C39-C40 & $-178.00(16)$ & Fe1-C38-C39-C40 & $-59.05(15)$ \\
\hline C42-C38-C39-Fe1 & $60.02(14)$ & P1-C38-C39-Fe1 & $-118.95(17)$ \\
\hline C38-C39-C40-C41 & $-0.8(3)$ & Fe1-C39-C40-C41 & $-58.94(16)$ \\
\hline C38-C39-C40-Fe1 & $58.17(15)$ & C39-C40-C41-C42 & $0.3(3)$ \\
\hline Fe1-C40-C41-C42 & $-58.49(15)$ & C39-C40-C41-Fe1 & $58.75(16)$ \\
\hline C40-C41-C42-C38 & $0.3(2)$ & Fe1-C41-C42-C38 & $-58.58(14)$ \\
\hline C40-C41-C42-Fe1 & $58.92(15)$ & C39-C38-C42-C41 & $-0.8(2)$ \\
\hline P1-C38-C42-C41 & $178.21(15)$ & Fe1-C38-C42-C41 & $59.45(15)$ \\
\hline C39-C38-C42-Fe1 & $-60.26(14)$ & P1-C38-C42-Fe1 & $118.76(15)$ \\
\hline C32-P2-C43-C47 & $12.3(2)$ & C26-P2-C43-C47 & $-92.56(19)$ \\
\hline Ni1-P2-C43-C47 & $138.21(17)$ & C32-P2-C43-C44 & $-162.99(15)$ \\
\hline C26-P2-C43-C44 & $92.12(16)$ & Ni1-P2-C43-C44 & $-37.11(17)$ \\
\hline C32-P2-C43-Fe1 & $-78.33(13)$ & C26-P2-C43-Fe1 & $176.79(11)$ \\
\hline Ni1-P2-C43-Fe1 & $47.55(13)$ & C47-C43-C44-C45 & $0.2(2)$ \\
\hline P2-C43-C44-C45 & $176.56(14)$ & Fe1-C43-C44-C45 & $60.13(14)$ \\
\hline C47-C43-C44-Fe1 & $-59.88(13)$ & $\mathrm{P} 2-\mathrm{C} 43-\mathrm{C} 44-\mathrm{Fe} 1$ & $116.43(14)$ \\
\hline C43-C44-C45-C46 & $-0.4(2)$ & Fe1-C44-C45-C46 & $58.22(15)$ \\
\hline $\mathrm{C} 43-\mathrm{C} 44-\mathrm{C}$ & $-58.65(13)$ & C44-C45-C46-C47 & $0.4(2)$ \\
\hline
\end{tabular}




$\begin{array}{llll}\text { Fe1-C45-C46-C47 } & 58.04(14) & \text { C44-C45-C46-Fe1 } & -57.60(14) \\ \text { C45-C46-C47-C43 } & -0.3(2) & \text { Fe1-C46-C47-C43 } & 58.74(13) \\ \text { C45-C46-C47-Fe1 } & -59.02(15) & \text { C44-C43-C47-C46 } & 0.0(2) \\ \text { P2-C43-C47-C46 } & -175.79(15) & \text { Fe1-C43-C47-C46 } & -59.87(14) \\ \text { C44-C43-C47-Fe1 } & 59.89(13) & \text { P2-C43-C47-Fe1 } & -115.92(17) \\ \text { C53-C48-C49-C50 } & 0 & \text { C48-C49-C50-C51 } & 0 \\ \text { C49-C50-C51-C52 } & 0 & \text { C50-C51-C52-C53 } & 0 \\ \text { C51-C52-C53-C48 } & 0 & \text { C49-C48-C53-C52 } & 0 \\ \text { C56-C54-C55-C56 } & 0.0(4) & \text { C54-C55-C56-C54 } & 0.0(4)\end{array}$

Table S9g. Anisotropic atomic displacement parameters $\left(\AA^{2}\right)$ for 8cc.

The anisotropic atomic displacement factor exponent takes the form: $-2 \pi^{2}[$ $\left.\mathrm{h}^{2} \mathrm{a}^{* 2} \mathrm{U}_{11}+\ldots+2 \mathrm{hka}{ }^{*} \mathrm{~b}^{*} \mathrm{U}_{12}\right]$

\begin{tabular}{|c|c|c|c|c|c|c|}
\hline & $\mathrm{U}_{11}$ & $\mathbf{U}_{22}$ & $\mathbf{U}_{33}$ & $\mathbf{U}_{23}$ & $\mathbf{U}_{13}$ & $\mathbf{U}_{12}$ \\
\hline Ni1 & $0.02175(12)$ & $0.01943(12)$ & $0.01717(11)$ & $0.00308(8)$ & $-0.00255(9)$ & $-0.00167(9)$ \\
\hline $\mathrm{Fe} 1$ & $0.02532(14)$ & $0.02846(15)$ & $0.01840(13)$ & $0.00717(10)$ & $-0.00122(10)$ & $0.00361(11)$ \\
\hline $\mathrm{P} 1$ & $0.0206(2)$ & $0.0224(2)$ & $0.0196(2)$ & $0.00122(17)$ & $-0.00191(17)$ & $0.00153(18)$ \\
\hline $\mathrm{P} 2$ & $0.0221(2)$ & $0.0198(2)$ & $0.0163(2)$ & $0.00259(17)$ & $-0.00064(17)$ & $-0.00068(17)$ \\
\hline O1 & $0.0263(7)$ & $0.0277(7)$ & $0.0217(6)$ & $0.0083(5)$ & $-0.0056(5)$ & $-0.0070(5)$ \\
\hline $\mathrm{C} 1$ & $0.0255(9)$ & $0.0218(9)$ & $0.0184(8)$ & $0.0008(7)$ & $-0.0029(7)$ & $-0.0003(7)$ \\
\hline $\mathrm{C} 2$ & $0.0268(9)$ & $0.0219(9)$ & $0.0222(9)$ & $0.0028(7)$ & $-0.0028(7)$ & $-0.0028(7)$ \\
\hline $\mathrm{C} 3$ & $0.0304(10)$ & $0.0284(10)$ & $0.0290(10)$ & $0.0052(8)$ & $-0.0052(8)$ & $-0.0063(8)$ \\
\hline $\mathrm{C} 4$ & $0.0495(14)$ & $0.0275(11)$ & $0.0353(12)$ & $0.0028(9)$ & $-0.0108(10)$ & $-0.0098(10)$ \\
\hline $\mathrm{C} 5$ & $0.076(2)$ & $0.0349(13)$ & $0.0484(15)$ & $-0.0016(11)$ & $0.0062(14)$ & $0.0043(13)$ \\
\hline C6 & $0.076(2)$ & $0.0434(16)$ & $0.091(3)$ & $-0.0081(16)$ & $-0.0384(19)$ & $-0.0176(15)$ \\
\hline $\mathrm{C} 7$ & $0.0276(9)$ & $0.0183(8)$ & $0.0218(9)$ & $0.0009(7)$ & $0.0014(7)$ & $-0.0006(7)$ \\
\hline $\mathrm{C} 8$ & $0.0284(10)$ & $0.0233(9)$ & $0.0236(9)$ & $0.0040(7)$ & $0.0031(8)$ & $0.0005(7)$ \\
\hline C9 & $0.0324(10)$ & $0.0289(10)$ & $0.0246(10)$ & $0.0040(8)$ & $-0.0018(8)$ & $0.0029(8)$ \\
\hline $\mathrm{C} 10$ & $0.0460(13)$ & $0.0297(11)$ & $0.0227(9)$ & $0.0046(8)$ & $0.0046(9)$ & $0.0018(9)$ \\
\hline $\mathrm{C} 11$ & $0.0432(12)$ & $0.0307(11)$ & $0.0294(10)$ & $0.0034(8)$ & $0.0114(9)$ & $-0.0078(9)$ \\
\hline
\end{tabular}




\begin{tabular}{|c|c|c|c|c|c|c|}
\hline & $\mathrm{U}_{11}$ & $\mathbf{U}_{22}$ & $\mathbf{U}_{33}$ & $\mathbf{U}_{23}$ & $\mathbf{U}_{13}$ & $\mathbf{U}_{12}$ \\
\hline $\mathrm{C} 12$ & $0.0318(11)$ & $0.0285(10)$ & $0.0297(10)$ & $0.0010(8)$ & $0.0033(8)$ & $-0.0064(8)$ \\
\hline $\mathrm{C} 13$ & $0.0594(16)$ & $0.0607(17)$ & $0.0265(11)$ & $0.0135(11)$ & $0.0047(11)$ & $0.0002(13)$ \\
\hline $\mathrm{C} 14$ & $0.0298(10)$ & $0.0277(10)$ & $0.0209(9)$ & $-0.0004(7)$ & $-0.0029(7)$ & $-0.0004(8)$ \\
\hline $\mathrm{C} 15$ & $0.0323(11)$ & $0.0250(10)$ & $0.0337(11)$ & $-0.0008(8)$ & $0.0001(9)$ & $-0.0017(8)$ \\
\hline $\mathrm{C} 16$ & $0.0493(14)$ & $0.0283(11)$ & $0.0367(12)$ & $-0.0049(9)$ & $0.0072(10)$ & $-0.0002(10)$ \\
\hline $\mathrm{C} 17$ & $0.0597(16)$ & $0.0414(13)$ & $0.0367(13)$ & $-0.0148(10)$ & $-0.0030(11)$ & $-0.0077(12)$ \\
\hline $\mathrm{C} 18$ & $0.0432(15)$ & $0.075(2)$ & $0.0672(19)$ & $-0.0343(17)$ & $-0.0176(14)$ & $-0.0043(14)$ \\
\hline $\mathrm{C} 19$ & $0.0302(12)$ & $0.0634(17)$ & $0.0571(16)$ & $-0.0284(14)$ & $-0.0116(11)$ & $0.0044(11)$ \\
\hline $\mathrm{C} 20$ & $0.0242(9)$ & $0.0233(9)$ & $0.0304(10)$ & $-0.0005(8)$ & $0.0009(8)$ & $0.0007(7)$ \\
\hline $\mathrm{C} 21$ & $0.0314(11)$ & $0.0422(12)$ & $0.0293(10)$ & $-0.0025(9)$ & $0.0023(9)$ & $0.0041(9)$ \\
\hline $\mathrm{C} 22$ & $0.0413(14)$ & $0.0709(19)$ & $0.0373(13)$ & $-0.0088(13)$ & $0.0123(11)$ & $0.0040(13)$ \\
\hline $\mathrm{C} 23$ & $0.0340(13)$ & $0.0560(16)$ & $0.0639(18)$ & $-0.0132(14)$ & $0.0171(12)$ & $0.0060(12)$ \\
\hline $\mathrm{C} 24$ & $0.0298(12)$ & $0.0443(14)$ & $0.0739(19)$ & $0.0070(13)$ & $0.0072(12)$ & $0.0124(10)$ \\
\hline $\mathrm{C} 25$ & $0.0281(11)$ & $0.0452(13)$ & $0.0467(13)$ & $0.0124(11)$ & $0.0028(10)$ & $0.0084(10)$ \\
\hline $\mathrm{C} 26$ & $0.0257(9)$ & $0.0181(8)$ & $0.0187(8)$ & $-0.0012(7)$ & $-0.0030(7)$ & $-0.0001(7)$ \\
\hline $\mathrm{C} 27$ & $0.0280(10)$ & $0.0279(10)$ & $0.0254(9)$ & $0.0063(8)$ & $-0.0005(8)$ & $-0.0013(8)$ \\
\hline $\mathrm{C} 28$ & $0.0254(10)$ & $0.0327(11)$ & $0.0409(12)$ & $0.0051(9)$ & $0.0017(9)$ & $-0.0017(8)$ \\
\hline $\mathrm{C} 29$ & $0.0270(10)$ & $0.0360(12)$ & $0.0434(12)$ & $0.0029(10)$ & $-0.0118(9)$ & $0.0003(9)$ \\
\hline $\mathrm{C} 30$ & $0.0399(12)$ & $0.0406(12)$ & $0.0266(10)$ & $0.0072(9)$ & $-0.0115(9)$ & $-0.0005(10)$ \\
\hline $\mathrm{C} 31$ & $0.0314(10)$ & $0.0323(10)$ & $0.0195(9)$ & $0.0019(8)$ & $-0.0011(8)$ & $-0.0009(8)$ \\
\hline C32 & $0.0288(10)$ & $0.0226(9)$ & $0.0183(8)$ & $0.0012(7)$ & $0.0017(7)$ & $0.0012(7)$ \\
\hline $\mathrm{C} 33$ & $0.0286(10)$ & $0.0294(10)$ & $0.0329(11)$ & $0.0024(8)$ & $0.0060(8)$ & $-0.0001(8)$ \\
\hline C34 & $0.0400(13)$ & $0.0401(13)$ & $0.0412(13)$ & $-0.0014(10)$ & $0.0162(10)$ & $0.0059(10)$ \\
\hline $\mathrm{C} 35$ & $0.0518(15)$ & $0.0347(12)$ & $0.0432(13)$ & $-0.0113(10)$ & $0.0135(11)$ & $0.0032(11)$ \\
\hline $\mathrm{C} 36$ & $0.0455(13)$ & $0.0312(11)$ & $0.0474(14)$ & $-0.0122(10)$ & $0.0112(11)$ & $-0.0063(10)$ \\
\hline C37 & $0.0325(11)$ & $0.0286(10)$ & $0.0340(11)$ & $-0.0047(9)$ & $0.0079(9)$ & $-0.0034(8)$ \\
\hline C38 & $0.0214(9)$ & $0.0298(10)$ & $0.0233(9)$ & $0.0055(8)$ & $-0.0024(7)$ & $0.0035(7)$ \\
\hline C39 & $0.0312(11)$ & $0.0484(13)$ & $0.0237(10)$ & $0.0055(9)$ & $-0.0081(8)$ & $-0.0012(10)$ \\
\hline $\mathrm{C} 40$ & $0.0282(11)$ & $0.0606(16)$ & $0.0331(11)$ & $0.0247(11)$ & $-0.0083(9)$ & $0.0027(10)$ \\
\hline
\end{tabular}




$\begin{array}{lllllll} & \mathbf{U}_{11} & \mathbf{U}_{22} & \mathbf{U}_{33} & \mathbf{U}_{\mathbf{2 3}} & \mathbf{U}_{13} & \mathbf{U}_{\mathbf{1 2}} \\ \mathrm{C} 41 & 0.0292(11) & 0.0365(12) & 0.0519(14) & 0.0221(11) & 0.0013(10) & 0.0087(9) \\ \mathrm{C} 42 & 0.0257(10) & 0.0274(10) & 0.0360(11) & 0.0075(9) & 0.0034(8) & 0.0059(8) \\ \mathrm{C} 43 & 0.0207(8) & 0.0235(9) & 0.0172(8) & 0.0045(7) & -0.0002(7) & 0.0018(7) \\ \mathrm{C} 44 & 0.0239(9) & 0.0283(10) & 0.0229(9) & 0.0022(7) & 0.0000(7) & 0.0042(7) \\ \mathrm{C} 45 & 0.0301(10) & 0.0420(12) & 0.0194(9) & 0.0029(8) & 0.0021(8) & 0.0048(9) \\ \mathrm{C} 46 & 0.0309(10) & 0.0375(11) & 0.0236(9) & 0.0107(8) & 0.0028(8) & 0.0033(9) \\ \mathrm{C} 47 & 0.0279(10) & 0.0263(10) & 0.0240(9) & 0.0070(8) & 0.0019(8) & -0.0002(8) \\ \mathrm{C} 48 & 0.110(3) & 0.089(3) & 0.071(2) & 0.002(2) & 0.003(2) & 0.039(3) \\ \mathrm{C} 49 & 0.075(3) & 0.087(3) & 0.093(3) & 0.019(2) & 0.015(2) & 0.016(2) \\ \mathrm{C} 50 & 0.083(3) & 0.102(4) & 0.135(4) & 0.035(3) & -0.021(3) & 0.000(3) \\ \mathrm{C} 51 & 0.078(3) & 0.111(4) & 0.169(5) & 0.045(4) & 0.001(3) & 0.016(3) \\ \mathrm{C} 52 & 0.065(3) & 0.093(4) & 0.320(10) & -0.014(5) & 0.015(4) & -0.005(3) \\ \mathrm{C} 53 & 0.078(3) & 0.119(4) & 0.195(6) & -0.058(4) & -0.042(3) & 0.032(3) \\ \mathrm{C} 54 & 0.0513(16) & 0.0459(15) & 0.069(2) & -0.0166(14) & -0.0211(14) & 0.0176(12) \\ \mathrm{C} 55 & 0.0607(18) & 0.0530(17) & 0.0549(16) & -0.0139(13) & -0.0092(14) & 0.0272(14) \\ \mathrm{C} 56 & 0.0646(18) & 0.0447(15) & 0.0582(17) & -0.0245(13) & -0.0269(14) & 0.0260(14)\end{array}$

Table S9h. Hydrogen atomic coordinates and isotropic atomic displacement parameters $\left(\AA^{2}\right)$ for

8cc.

$\begin{array}{lllll} & \mathbf{x} / \mathbf{a} & \mathbf{y} / \mathbf{b} & \mathbf{z} / \mathbf{c} & \mathbf{U}(\mathbf{e q}) \\ \text { H3A } & 0.1626 & 0.4963 & 0.6415 & 0.036 \\ \text { H3B } & 0.1189 & 0.4731 & 0.7176 & 0.036 \\ \text { H4 } & 0.1791 & 0.3448 & 0.7170 & 0.046 \\ \text { H5A } & 0.2567 & 0.3017 & 0.6141 & 0.079 \\ \text { H5B } & 0.3068 & 0.3688 & 0.6638 & 0.079 \\ \text { H5C } & 0.2588 & 0.3917 & 0.5840 & 0.079 \\ \text { H6A } & 0.0952 & 0.3796 & 0.5721 & 0.108 \\ \text { H6B } & 0.0472 & 0.3618 & 0.6474 & 0.108\end{array}$




\begin{tabular}{|c|c|c|c|c|}
\hline & $\mathbf{x} / \mathbf{a}$ & $\mathbf{y} / \mathbf{b}$ & $\mathbf{z} / \mathbf{c}$ & $\mathbf{U}(\mathbf{e q})$ \\
\hline $\mathrm{H} 6 \mathrm{C}$ & 0.1011 & 0.2930 & 0.6102 & 0.108 \\
\hline H8 & 0.3935 & 0.4958 & 0.8209 & 0.03 \\
\hline H9 & 0.4239 & 0.4546 & 0.9450 & 0.035 \\
\hline H11 & 0.1823 & 0.3807 & 0.9566 & 0.041 \\
\hline H12 & 0.1508 & 0.4204 & 0.8320 & 0.036 \\
\hline $\mathrm{H} 13 \mathrm{~A}$ & 0.2818 & 0.3538 & 1.0624 & 0.073 \\
\hline H13B & 0.3334 & 0.4349 & 1.0787 & 0.073 \\
\hline $\mathrm{H} 13 \mathrm{C}$ & 0.3811 & 0.3580 & 1.0486 & 0.073 \\
\hline H15 & 0.3023 & 0.5382 & 0.4992 & 0.037 \\
\hline H16 & 0.2760 & 0.4347 & 0.4126 & 0.045 \\
\hline H17 & 0.1383 & 0.4111 & 0.3612 & 0.055 \\
\hline H18 & 0.0272 & 0.4895 & 0.3974 & 0.075 \\
\hline H19 & 0.0526 & 0.5941 & 0.4838 & 0.061 \\
\hline $\mathrm{H} 21$ & 0.1471 & 0.6358 & 0.7109 & 0.041 \\
\hline H22 & 0.0276 & 0.6733 & 0.7736 & 0.059 \\
\hline $\mathrm{H} 23$ & -0.0827 & 0.7431 & 0.7089 & 0.061 \\
\hline $\mathrm{H} 24$ & -0.0736 & 0.7755 & 0.5817 & 0.059 \\
\hline $\mathrm{H} 25$ & 0.0466 & 0.7405 & 0.5190 & 0.048 \\
\hline $\mathrm{H} 27$ & 0.5752 & 0.7643 & 0.5645 & 0.033 \\
\hline $\mathrm{H} 28$ & 0.7153 & 0.7436 & 0.6117 & 0.04 \\
\hline H29 & 0.7434 & 0.6909 & 0.7331 & 0.043 \\
\hline H30 & 0.6314 & 0.6563 & 0.8060 & 0.044 \\
\hline H31 & 0.4909 & 0.6756 & 0.7593 & 0.033 \\
\hline H33 & 0.2683 & 0.7916 & 0.7069 & 0.036 \\
\hline H34 & 0.2404 & 0.9025 & 0.7814 & 0.048 \\
\hline H35 & 0.3377 & 1.0069 & 0.7933 & 0.051 \\
\hline H36 & 0.4654 & 0.9998 & 0.7343 & 0.049 \\
\hline H37 & 0.4952 & 0.8879 & 0.6617 & 0.038 \\
\hline H39 & 0.1975 & 0.6883 & 0.3828 & 0.042 \\
\hline
\end{tabular}




$\begin{array}{lllll} & \mathbf{x} / \mathbf{a} & \mathbf{y} / \mathbf{b} & \mathbf{z} / \mathbf{c} & \mathbf{U}(\mathbf{e q}) \\ \text { H40 } & 0.2088 & 0.8318 & 0.3306 & 0.049 \\ \text { H41 } & 0.2227 & 0.9298 & 0.4447 & 0.047 \\ \text { H42 } & 0.2210 & 0.8466 & 0.5686 & 0.036 \\ \text { H44 } & 0.4064 & 0.6512 & 0.4721 & 0.03 \\ \text { H45 } & 0.4124 & 0.7272 & 0.3448 & 0.037 \\ \text { H46 } & 0.4247 & 0.8778 & 0.3739 & 0.037 \\ \text { H47 } & 0.4276 & 0.8974 & 0.5202 & 0.031 \\ \text { H48 } & 0.0169 & 0.1611 & 0.5692 & 0.108 \\ \text { H49 } & 0.1486 & 0.1619 & 0.6392 & 0.101 \\ \text { H50 } & 0.2007 & 0.0449 & 0.6977 & 0.129 \\ \text { H51 } & 0.1213 & -0.0729 & 0.6862 & 0.144 \\ \text { H52 } & -0.0103 & -0.0737 & 0.6161 & 0.191 \\ \text { H53 } & -0.0625 & 0.0433 & 0.5577 & 0.16 \\ \text { H54 } & 0.5944 & 0.6070 & 0.4978 & 0.068 \\ \text { H55 } & 0.5536 & 0.5386 & 0.3856 & 0.068 \\ \text { H56 } & 0.4596 & 0.4322 & 0.3885 & 0.069\end{array}$




\section{References}

(1) Montgomery, J. Science of Synthesis 2001, 11, 61.

(2) Taylor, E. C.; McKillop, A.; Hawks, G. Organic Syntheses 1972, 52, 36.

(3) Staudaher, N. D.; Lovelace, J.; Johnson, M. P.; Louie, J. Organic Syntheses, Manuscript in Review.

(4) Le Page, M. D.; Patrick, B. O.; Rettig, S. J.; James, B. R. Inorg. Chem. Acta. 2015, 431, 276.

(5) Chavez, C. A.; Choi, J.; Nesterov, E. E. Macromolecules 2014, 47, 506.

(6) Yin, G.; Kalvet, I.; Englert, U.; Schoenebeck, F. J. Am. Chem. Soc. 2015, 137, 4164.

(7) Frisch, M. J.; Trucks, G. W.; Schlegel, H. B.; Scuseria, G. E.; Robb, M. A.; Cheeseman, J. R.; Scalmani, G.; Barone, V.; Mennucci, B.; Petersson, G. A.; Nakatsuji, H.; Caricato, M.; Li, X.; Hratchian, H. P.; Izmaylov, A. F.; Bloino, J.; Zheng, G.; Sonnenberg, J. L.; Hada, M.; Ehara, M.; Toyota, K.; Fukuda, R.; Hasegawa, J.; Ishida, M.; Nakajima, T.; Honda, Y.; Kitao, O.; Nakai, H.; Vreven, T.; Montgomery Jr., J. A.; Peralta, J. E.; Ogliaro, F.; Bearpark, M. J.; Heyd, J.; Brothers, E. N.; Kudin, K. N.; Staroverov, V. N.; Kobayashi, R.; Normand, J.; Raghavachari, K.; Rendell, A. P.; Burant, J. C.; Iyengar, S. S.; Tomasi, J.; Cossi, M.; Rega, N.; Millam, N. J.; Klene, M.; Knox, J. E.; Cross, J. B.; Bakken, V.; Adamo, C.; Jaramillo, J.; Gomperts, R.; Stratmann, R. E.; Yazyev, O.; Austin, A. J.; Cammi, R.; Pomelli, C.; Ochterski, J. W.; Martin, R. L.; Morokuma, K.; Zakrzewski, V. G.; Voth, G. A.; Salvador, P.; Dannenberg, J. J.; Dapprich, S.; Daniels, A. D.; Farkas, Ö.; Foresman, J. B.; Ortiz, J. V.; Cioslowski, J.; Fox, D. J.; Gaussian, Inc.: Wallingford, CT, USA, 2009.

(8) (a) Becke, A. D. J. Chem. Phys. 1993, 98, 5648. (b) Lee, C.; Yang, W.; Parr, R. G. Phys. Rev. B 1988, 37, 785.

(9) (a) Schaefer, A.; Horn, H.; Ahlrichs, R. J. Chem. Phys. 1992, 97, 2571. (b) Schaefer, A.; Huber, C.; Ahlrichs, R. J. Chem. Phys. 1994, 100, 1994.

(10) (a) Becke, A. D. Phys. Rev. A 1988, 38, 3098. (b) Perdew, J. P. Phys. Rev. B 1986, 33, 8822.

(11) Glendening, E. D.; Reed, A. E.; Carpenter, J. E.; Weinhold, F. NBO Version 3.1.

(12) Tenderholt, A. L. QMForge, Version 2.4, http://amforge.sourceforge.net.

(13) (a) Szentpaly, L. V.; Fuentealba, P.; Preuss, H.; Stoll, H. Chem. Phys. Lett. 1982, 93, 1982. (b) Dolg, M.; Wedig, U.; Stoll, H.; Preuss, H. J. Chem. Phys. 1987, 86, 866. (c) Schwerdtfeger, P.; Dolg, M.; Schwarz, W. H. E.; Bowmaker, G. A.; Boyd, P. D. W. J. Chem. Phys. 1989, 91, 1762.

(14) (a) Zhao, Y.; Truhlar, D. G. Theor. Chem. Acc. 2008, 120, 215. (b) Zhao, Y.; Truhlar, D. G. Acc. Chem. Res. 2008, 41, 157.

(15) Kumar, P.; Thakur, A.; Hong, X.; Houk, K. N.; Louie, J. J. Am. Chem. Soc. 2014, 136, 17844. 